2013-09-13

\title{
Surface-consistent matching filters for time-lapse processing
}

\author{
AL MUTLAQ, MAHDI
}

AL MUTLAQ, MAHDI. (2013). Surface-consistent matching filters for time-lapse processing (Unpublished doctoral thesis). University of Calgary, Calgary, AB. doi:10.11575/PRISM/27940 http://hdl.handle.net/11023/968

Downloaded from PRISM Repository, University of Calgary 


\title{
UNIVERSITY OF CALGARY
}

Surface-consistent matching filters for time-lapse processing

by

Mahdi H. Al Mutlaq

\begin{abstract}
A DISSERTATION
SUBMITTED TO THE FACULTY OF GRADUATE STUDIES

IN PARTIAL FULFILLMENT OF THE REQUIREMENTS FOR THE DEGREE OF DOCTOR OF PHILOSOPHY
\end{abstract}

DEPARTMENT OF GEOSCIENCE

CALGARY, ALBERTA

AUGUST, 2013

(C) Mahdi H. Al Mutlaq 2013 


\section{Abstract}

The problem of mismatch between repeated time-lapse seismic surveys remains a challenge, particularly for land acquisition. In this dissertation, we present a new algorithm, which is an extension of the surface-consistent model, and which minimizes the mismatch between surveys, hence improving repeatability.

We introduce the concept of surface-consistent matching filters (SCMF) for processing time-lapse seismic data, where matching filters are convolutional filters that minimize the sum-squared error between two signals. Since in the Fourier domain, a matching filter is the spectral ratio of the two signals, we extend the well known surface-consistent hypothesis such that the data term is a trace-by-trace spectral ratio of two datasets instead of only one (i.e. surface-consistent deconvolution). To avoid unstable division of spectra, we compute the spectral ratios in the time domain by first designing tracesequential, least-squares matching filters, then Fourier transforming them. A subsequent least-squares solution then factors the trace-sequential matching filters into four operators: two surface-consistent (source and receiver), and two subsurface-consistent (offset and midpoint).

We apply the algorithm to two datasets: a synthetic time-lapse model and field data from a $\mathrm{CO}_{2}$ monitoring site in Northern Alberta. In addition, two common time-lapse processing schemes (independent processing and simultaneous processing) are compared. We present a modification of the simultaneous processing scheme as a direct result of applying the new SCMF algorithm. The results of applying the SCMF together with the new modified simultaneous processing flow reveal the potential benefit of the method, however some challenges remain, specifically in the presence of random noise. 


\section{Acknowledgements}

During my PhD program, I had the privilege to work with Dr. Gary Margrave. Gary's attention to scientific details (geophysics, math, and programming) greatly influenced me, and this thesis owes much of his contribution, guidance and encouragement. I could not have imagined having a better advisor and mentor for my PhD study.

I would also like to acknowledge my thesis committee: Dr. Larry Lines, Dr. Don Lawton, Dr. Wenyuan Liao, and Dr. Peter Cary, for their support, and insightful comments and questions.

I benefited greatly from the environment provided to me by the CREWES project which is made possible by the continuous support of the all the sponsors, and for that I say thank you. My gratitude to one of the sponsors, my employer Saudi Aramco, who gave me this opportunity and enabled me to complete my studies without adversity. There are also many individuals in Saudi Aramco, too many to name, who influenced my geophysical understanding and supported me during my study and for that I say thank you.

Mike Hall and Judy Leah from CGG are acknowledged for their work and useful discussions on noise attenuation of the field data set.

The geophysics courses and the scientific discussions I had with Dr. Krebes, Dr. Ferguson, Dr. Lawton, Dr. Innanen, Dr. Lines and Dr. Margrave provided me with much of the knowledge required to complete my PhD program and for that I thank them. I also would like to thank all of my colleagues in the CREWES project (I am hoping not to forget anyone): Kevin Hall, Helen Issac, Pat Daley, Laura Baird, Rolf Maier, Marcus Wilson, Mohammed Alarfaj, Nasser Yousefzadeh, Roohollah Askari, Vladimeir Zubov, Raul Cova, Heather Lloyd, Hassan Khaniani, Diane Lespinasse, Patricia Gavotti, Haitham Hamid, Nassir Saeed, and Steve Kim. Two special people are greatly acknowl- 
edged: Dave Henley and Faranak Mahmoudian for their constant support, meaningful discussions, and most of all their friendship.

I also would like to thank all of my friends outside the geoscience world, particularly my countrymen who stood by my side all the times. It is really difficult to name them all, but they will always be in my heart.

Finally, this work would not have been possible if it was not for the continuous support I received from all of my family and friends, especially my lovely wife, my dearest mother, and my beautiful kids. I am indebted to their support, encouragement, and love. 


\section{Table of Contents}

Abstract ............................ i

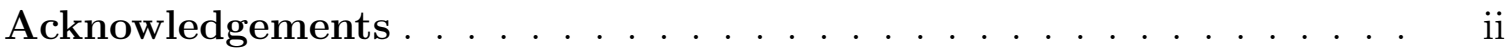

Table of Contents . . . . . . . . . . . . . . . . . . . . iv

List of Tables . . . . . . . . . . . . . . . . . . . . . . v vi

List of Figures . . . . . . . . . . . . . . . . . . . v vii

List of Symbols . . . . . . . . . . . . . . . . . . . . . xiv

$1 \quad$ Introduction . . . . . . . . . . . . . . . . . . . . 1

1.1 Reservoir monitoring . . . . . . . . . . . . . . . . . . . . . . . 1

1.2 Time-lapse seismic: acquisition and processing . . . . . . . . . . 2

1.2 .1 Acquisition repeatability . . . . . . . . . . . . . . 3

1.2 .2 Processing repeatability . . . . . . . . . . . . . . . . 4

1.2 .3 Remaining challenges . . . . . . . . . . . . . . . . 6

1.3 Dissertation contributions . . . . . . . . . . . . . . . . . . . . . . 11

1.4 Dissertation organization . . . . . . . . . . . . . . . . . . . . . . . . . 12

2 The surface-consistent model . . . . . . . . . . . . . . . . . . . . . 13

2.1 Introduction . . . . . . . . . . . . . . . . . . . . . . . . . . 13

2.2 The surface-consistent hypothesis . . . . . . . . . . . . . . . . . . . . . . 13

2.2 .1 The surface diagram . . . . . . . . . . . . . . . . . . . . . . 14

2.2 .2 Basic assumptions . . . . . . . . . . . . . . . . . . . . . 15

2.2 .3 General equation . . . . . . . . . . . . . . . . . . . . 16

2.3 Applications in seismic data processing . . . . . . . . . . . . . . . . 18

2.3.1 Residual static corrections . . . . . . . . . . . . . . . . . . 18

2.3 .2 Deconvolution . . . . . . . . . . . . . . . . . . . . . . . . 29

2.3.3 Other applications . . . . . . . . . . . . . . . . . . 32

2.4 Accuracy of the model $\ldots \ldots \ldots \ldots$

2.5 Summary . . . . . . . . . . . . . . . . . . . . . . . . 33

3 Extending the surface-consistent hypothesis to model two seismic surveys 38

3.1 Introduction . . . . . . . . . . . . . . . . . . . . . . 38

3.2 Extending the surface-consistent hypothesis to model two seismic surveys 39

3.2.1 Frequency-domain form of the extended model . . . . . . . . . . 40

3.2 .2 Analogy to matching filters . . . . . . . . . . . . . . . 40

3.2 .3 SCMF: time-domain LSQ vs spectral ratio . . . . . . . . . . 52

3.3 Surface-consistent matching filter decomposition . . . . . . . . . . . . 54

3.4 Summary . . . . . . . . . . . . . . . . . . . . . . 56

4 SCMF: application to a time-lapse model experiment . . . . . . . . 58

4.1 Introduction . . . . . . . . . . . . . . . . . . . . . . . . . . . . 58

4.2 Time-lapse model experiment . . . . . . . . . . . . . . . . . . . . . . 62

4.2.1 Computing surface-consistent matching filters . . . . . . . . 67

$4.3 \quad$ Filter application and analysis . . . . . . . . . . . . . . . . 72

4.3.1 Trace-by-trace (non-surface-consistent) and Surface-consistent matching filters . . . . . . . . . . . . . . 73 
4.3.2 The effects of the surface-consistent terms . . . . . . . . . 76

4.4 Summary . . . . . . . . . . . . . . . . . . . . . 78

5 SCMF: application to a $\mathrm{CO}_{2}$ monitoring study . . . . . . . . . 86

5.1 Introduction . . . . . . . . . . . . . . . . . . . . . . . . . . . . . . . . . .

5.2 Violet Grove $\mathrm{CO}_{2}$ pilot study . . . . . . . . . . . . . . . . . . 92

5.2.1 Geology of the area . . . . . . . . . . . . . . . . . 93

5.2.2 Geophysics data: acquisition and previous processing . . . . . . 97

5.3 Re-processing the Violet Grove data: prestack study . . . . . . . . . . . . 104

5.3.1 Pre-processing steps . . . . . . . . . . . . . . . . 104

5.3.2 Parallel processing . . . . . . . . . . . . . . . . . 108

$5.3 .3 \mathrm{SCMF} \ldots \ldots \ldots \ldots \ldots \ldots 113$

5.3.4 Simultaneous processing . . . . . . . . . . . 116

5.4 Poststack Analysis of the processed data . . . . . . . . . . . . . . . 125

5.4.1 Parallel processing ....................... 125

5.4.2 Simultaneous processing . . . . . . . . . . . . 126

5.4.3 Poststack matching . . . . . . . . . . . . . . . . . . . . 127

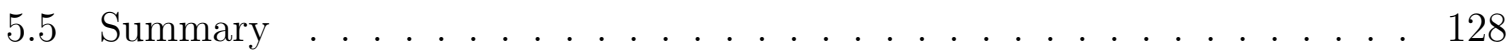

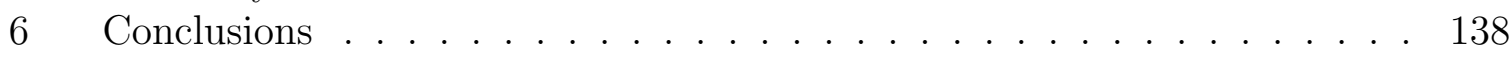




\section{List of Tables}

3.1 Match filter accuracy for the two noise-free traces in Figure 3.1 comparing the time-domain least-squares and the spectral ratio for five filter lengths. 46

3.2 Match filter accuracy for two noisy traces shown in Figure 3.5 comparing the time-domain least-squares and the spectral ratio for five filter lengths. $\quad 48$

5.1 Physical properties of the Cardium Formation from the study area (Hitchon, 2009). . . . . . . . . . . . . . . . . . . 95

5.2 Summary of the seismic program at the $\mathrm{CO}_{2}$-EOR study site (Alshuhail et al., 2008) . . . . . . . . . . . . . . . . . . . . . . . . . . 98

5.3 CGGVeritas processing work flow of the 2D P-wave surface seismic data set (Lawton et al., 2005; Alshuhail, 2011). . . . . . . . . . . . . . . 99

5.4 Parallel processing flow of Line 1 P-wave surface seismic data set. . . . . 110

5.5 Simultaneous processing flow of Line 1 P-wave surface seismic data set. . 119 


\section{List of Figures and Illustrations}

1.1 Two simple earth models (m/s) representing baseline (a), monitor $(\mathrm{b})$, and their time-lapse difference (c). The only difference is in the reservoir slab depicting a subsurface change due to reservoir condition change. . . . . .

1.2 Synthetic seismic data corresponding to the velocity model in Figure 1.1. (a) is the baseline seismic, (b) is the monitor seismic (same amplitude scaling as baseline), and their time-lapse difference (scaled by 5 ) is in (c). Note the difference is only at the reservoir level as expected. . . . . . . .

1.3 Two earth velocity models (units in $\mathrm{m} / \mathrm{s}$ ) representing baseline (a), monitor (b), and their time-lapse difference (c). Note that both models have both near surface and subsurface variations depicting a real land time-lapse

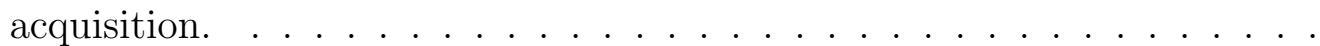

1.4 Synthetic seismic data corresponding to the velocity model in Figure 1.3. (a) is the baseline seismic, (b) is the monitor seismic, and their timelapse difference is in (c). All plots have the same amplitude scaling as the

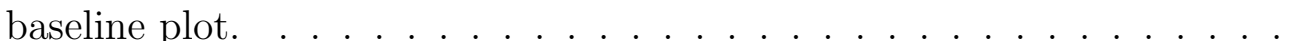

2.1 Surface diagram and principal trace organization. . . . . . . . . . . .

2.2 Schematic diagram depicting the earth and a ray path from a source to a receiver showing the concept of static corrections. (a) The original recorded data with the source $(S)$ and receiver $(R)$ on the surface of the earth. (b) The concept of static corrections with a pseudo datum plane below the weathering zone and a new source (S') and a new receiver (R'). . . . . .

2.3 Common-midpoint stacked sections showing the seismic data with (a) datum statics only and the same one with (b) datum statics and residual static corrections applied.

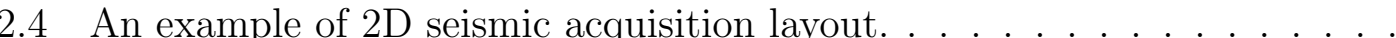

2.5 Geometry matrix of the synthetic data where the columns represent the sources, receivers, offsets, and midpoints. The rows represents the total number of traces. . . . . . . . . . . . . . . . . 25

2.6 CMP stacks before and after surface-consistent residual statics. . . . . . .

2.7 Stacked CMP sections showing the difference between single channel deconvolution in (a) compared to multichannel surface-consistent deconvolution in (b) (Hart, 1997). . . . . . . . . . . . . . .

2.8 Forward modeling of the surface-consistent model with variations in the source term only. . . . . . . . . . . . . . . . . . . .

2.9 Inverse modeling of the surface-consistent model. Deconvolving the same seismic traces in Figure 2.8 results in a different model than the input model of the forward operation. There is a leakage of the solution to other components. . . . . . . . . . . . . . . . . . 
2.10 Seismic data of the forward model (a), and seismic data of the inverse model (b), and their difference. Note the difference is minimum even though the two models are different. . . . . . . . . . . . . . . .

2.11 The sum squares of the residual of the traces generated from the input model (Figure 2.8) and the output traces generated from the output model (Figure 2.9) . . . . . . . . . . . . . . . . . . . .

2.12 Comparisons of the two adjacent filters of the input model (Figure 2.8) and
the output model (Figure 2.9). (a) is the source ratio, (b) is the receiver 35 ratio, (c) is the offset ratio, and $(\mathrm{d})$ is the midpoint ratio. . . . . . . . .

3.1 An example of two noise-free traces generated from the same reflectivity sequence but convolved with two different wavelets. The residual reflects the time-shift and the amplitude difference. . . . . . . . . . . . . .

3.2 The same example shown in Figure 3.1 but after time-domain matching of Trace 2 to Trace 1 where the result is Trace $1_{M F T} . \ldots . . . .$.

3.3 The same example shown in Figure 3.1 but after spectral ratio match filtering of Trace 2 to Trace 1 where the result is Trace $1_{M F F}$. . . . . . .

3.4 An example of three filter lengths used for the frequency-domain match filter. . . . . . . . . . . . . . . . . . . .

3.5 The same example shown in Figure 3.1 with added noise. . . . . . . . . .

3.6 The time-domain matching of Trace 2 to Trace 1 where the result is Trace $1_{M F T} \ldots \ldots \ldots \ldots \ldots \ldots \ldots$

3.7 The spectral ratio match filtering of Trace 2 to Trace 1 where the result is Trace $1_{M F F} \ldots \ldots \ldots \ldots \ldots$

3.8 Amplitude spectrum of Trace 1 (top) and Trace 2 (bottom) from Figure 3.5. Note that the signal bandwidth is different for both traces (even after bandlimiting the traces, difference will still exist). The horizontal lines define the approximate noise level of the spectrum. . . . . . . . . .

3.9 Processing workflow for the trace-by-trace surface-consistent matching filters. . . . . . . . . . . . . . . . . . .

3.10 Matrix structure of the system of linear equation described in equation 3.13 ; the number of columns of $\mathbf{G}=$ number of sources + number of unique receivers + number of unique offsets + number of midpoints, and the number of rows of $\mathbf{G}=$ total number of traces; the length of $\mathbf{d}=$ total number of traces. . . . . . . . . . . . . . . . .

4.1 Two earth velocity models (units in $\mathrm{m} / \mathrm{s}$ ) representing baseline (a), monitor (b), and their time-lapse difference (c). Note that both models have both near surface (showing effects of dry vs wet climate) and in the reservoir unit. . . . . . . . . . . . . . . . . . . .

4.2 Horizontal profile of near-surface attenuation for baseline model (gray line) and monitor model (black line)(a), source strength (b), and receiver coupling $(\mathrm{c}) \ldots \ldots \ldots \ldots \ldots \ldots$ 
4.3 Two raw shot records, source location at $1250 \mathrm{~m}$, from (a) the baseline survey and (b) the monitor survey. . . . . . . . . . . . . . . .

4.4 Two shot records, source location at $1250 \mathrm{~m}$, from the baseline survey (a) and the monitor survey (b). (c) shows the difference between (a) and (b), and (d) shows the NRMS computed between the two dotted lines (the analysis window). Note the mean NRMS is $144.7 \%$ which means both surveys are highly dissimilar. Black color on all seismic plots represents peaks and white represents troughs. All seismic displays have the same amplitude clipping as the baseline figure. . . . . . . . . . . . . .

4.5 Baseline shot (a), similar to Figure 4.4a, and monitor shot with $+26 \mathrm{~ms}$ time shift (b). The difference of (a) and (b) is shown is (c), and (d) shows the NRMS computed between the two dotted lines (the analysis window) of the difference plot. Note that the mean NRMS has decreased to $41.4 \%$ but there is a significant amplitude difference. . . . . . . . . . . . . .

4.6 NRMS sensitivity tests where (a) is NRMS versus time shift for three dominant frequencies and (b) is NRMS versus amplitude difference between two noise free traces. . . . . . . . . . . . . . . . . . .

4.7 In (a) is the log amplitude spectra of the source filters computed using equation 3.14. In (b) is a comparison of the average over frequency of the shot filters, referred to as source filters strength, in black line versus the ratio of the baseline survey source strength to the monitor survey source strength in gray line. The log amplitude spectra of the receiver components is shown in (c) and in (d) is a comparison of the receiver filter strength (black line) versus the receiver strength ratio of both surveys (light gray line). . . . . . . . . . . . . . . . . . .

4.8 In (a) is the log amplitude spectra of the offset filters and in (b) is the offset filter strengths computed as the average over frequency. Similarly, in (c) is the log amplitude spectra of the midpoint filters and in (b) is the midpoint filter strengths computed as the average over frequency. . . . .

4.9 Baseline shot shown in (a), the monitor shot after applying the fourcomponent filters to it in (b) (hereafter we named it matched monitor survey). The difference is shown in (c) and the computed NRMS between the two dashed lines is shown in (d). Note that the mean NRMS is down to $26.8 \%$. All plots have the same gain as the baseline. . . . . . . . . . .

4.10 In (a) is a difference plot between baseline shot and a trace-by-trace matched monitor shot. (b) stack of the difference between baseline and a trace-by-trace matched monitor. . . . . . . . . . . . . . . . . . . .

4.11 (a) is a prestack difference of the baseline and the matched monitor, with source and receiver terms applied, and (b) shows the poststack difference. (c) is a prestack difference with three terms applied (source, receiver and offset) also in (d) is the poststack difference. (e) is a prestack difference plot with four-term filters applied and (f) is the poststack difference. . . . 
4.12 In (a) is the migrated difference plot between baseline survey and monitor survey with a poststack time shift of $35 \mathrm{~ms}$ and in (b) is the migrated difference stack between baseline and four-terms surface-consistent matched monitor. . . . . . . . . . . . . . . . . . .

4.13 Average amplitude spectra of migrated stacks of baseline survey (black line), monitor survey (gray line), and matched monitor (dashed line) computed from all traces inside the window of analysis. Amplitude spectrum of the matched monitor survey matches nicely the baseline amplitude spectrum. . . . . . . . . . . . . . . . .

4.14 NRMS difference after different stages of processing for two different data sets. The light gray line represent a published example of real data set from Gulf of Mexico by Helgerud et al. (2011) where at raw stack stage the NRMS is about $70 \%$ and at final stack they reported an improvement of about $28 \%$. In our modeled data set we started with NRMS of about $144.7 \%$ in prestack stage, then about $70 \%$ at raw stack, then a significant improvement after the four-component surface-consistent matching filters and after final stack the NRMS is about $13.6 \%$. . . . . . . . . .

5.1 Top panels show a cross section from the time-lapse 3D seismic surveys at the Sleipner field. Note the strong $\mathrm{CO}_{2}$ plume signature on the 2001 survey onwards. Bottom panels show map view of the total reflection amplitude of the plume (Williams and Chadwick, 2012). . . . . . . . . . . . . .

5.2 A NW-SE oriented migrated P-wave section from the baseline (a) and the 2001 monitor (b) showing well resolved Marly and Vuggy zones and increasing amplitude signature due to the injected gas $(\mathrm{Li}, 2003)$. . . . .

5.3 (a) shows a time-lapse amplitude difference map extracted from the upper reservoir unit and (b) shows time-delays present in time-lapse difference map for the deep Bakken formation (White, 2009). . . . . . . . . . . .

5.4 Map of Alberta showing the location of the Pembina Oil Field and the $\mathrm{CO}_{2}$-EOR pilot site at Violet Grove (Dashtgard et al., 2006). . . . . . . .

5.5 General stratigraphic column of central Alberta of the Lower and Upper Cretaceous (Bachu and Bennion, 2008) shown in (a) and a typical well log from the Pembina Field showing the Cardium Formation in (b); a modified plot of that in Patterson (1957). . . . . . . . . . . . . . . .

5.6 Zero-offset seismic modeling of the effects of $\mathrm{CO}_{2}$ saturation in the Cardium reservoir. (Alshuhail et al., 2008). . . . . . . . . . . . . . .

5.7 Layout of the surface seismic lines, 3D seismic coverage (indicated by the dashed rectangle), and location of the injector wells, observation well, and VSP (Lawton et al., 2005). . . . . . . . . . . . . . . . . . .

$5.8 \mathrm{P}$-wave seismic correlation at well $102 / 7-11-48-9$. The blue trace is synthetic seismogram, and the red trace is extracted from the surface seismic at the well location $($ Chen, 2006). . . . . . . . . . . 
5.9 Processed P-wave data from Line 1 (Alshuhail et al., 2008). Top is Phase I (baseline), middle is Phase III (monitor) after poststack match filter, and at the bottom is the difference. The red line is the projection of the 102/7-11-48-9 well. . . . . . . . . . . . . . . . . . . .

5.10 Walkaway P-wave VSP data from Line 1 at the observation well (Alshuhail et al., 2008). On the left is Phase I , middle is Phase III, and on the right is the difference. . . . . . . . . . . . . . . . . .

5.11 Line 1 shot location for the baseline survey, in red, and the monitor survey, in blue, displayed in the left panel. Only the common shot locations were processed for time-lapse as shown in the right panel. . . . . . . . . . .

5.12 A raw shot record from the middle of Line 1. (a) shows the baseline, (b) shows the monitor, (c) shows the difference, and (d) shows the computed NRMS error. Red and yellow color mean high NRMS errors whereas blue means low NRMS errors. An AGC filter with $500 \mathrm{~ms}$ length is applied for display purposes. Plots (b) and (c) have the same amplitude scaling as plot (a)

5.13 Data after amplitude recovery compensation and linear noise attenuation. (a) shows the baseline survey, (b) shows the monitor survey, (c) shows the difference, and (d) shows the NRMS error. Note the clear difference in amplitude strengths between the base and the monitor as well as the nonrepeatability of the high amplitude (HA) noise indicated by green. Plots (b) and (c) have the same amplitude scaling as plot (a). . . . . . .

5.14 The baseline shot (a) and the monitor shot (b) after independent SCSD and independent SCAC. The difference is shown in (c) and the NRMS error is in (d). Plots (b) and (c) have the same amplitude scaling as plot (a). . . . . . . . . . . . . . . . . . .

5.15 Final velocity analysis for CMP 135. . . . . . . . . . . . .

5.16 Computed source filters shown in (a) and the time-average of the source filters magnitude in (b). In (c) is the receiver filters and their mean mag-

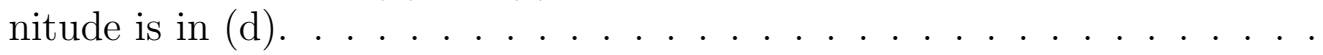

5.17 Computed offset filters shown in (a) and their mean magnitude for each offset in (b). (c) shows the midpoint filters, and their mean magnitude is in $(d)$.

5.18 The surface-consistent time-shifts for the source terms (a) and the receiver terms (b). . . . . . . . . . . . . . . . . 116

5.19 The baseline shot (a) and the monitor shot after applying the four-components SCMF (b). The difference is shown in (c) and the NRMS error is in (d). Note that the difference observed in Figure 5.13(c) is minimized after applying the SCMF, with the exception of the near-offset noise. Plots (b) and (c) have the same amplitude scaling as plot (a). . . . . . . . .

5.20 A zoom on the match filter window for the baseline (a), monitor (b), SCMF monitor (c), difference before $\operatorname{SCMF}(d)$, and difference after applying SCMF (e). All plots have the same amplitude scaling as the baseline. . . 
5.21 (a) shows the NRMS error computed from the baseline and the monitor without SCMF, and (b) shows the NRMS error after applying SCMF to the monitor survey. . . . . . . . . . . . . . . . .

5.22 Simultaneous surface-consistent deconvolution for baseline (a), matched monitor (b), difference (c), and the NRMS error (d). A bandpass filter $(4-8-90-100 \mathrm{~Hz})$ is applied for display. Plots (b) and (c) have the same amplitude scaling as plot (a). . . . . . . . . . . . . .

5.23 Simultaneous surface-consistent amplitude corrections (SCAC) and their corresponding fold. The panels show the SCAC for sources-term (a), the source fold (b), the SCAC for the receivers-term (c), the receiver fold (d), the SCAC for offsets-term (e), the offset fold (f), the SCAC for the midpoints (g), and the midpoint fold (h) . . . . . . . . . . . . 123

5.24 Simultaneous surface-consistent amplitude correction for baseline (a), matched monitor (b), difference (c), and the NRMS error (d). A bandpass filter $(8-12-55-70 \mathrm{~Hz})$ is applied for display. Plots (b) and (c) have the same amplitude scaling as plot (a).

5.25 Parallel processing stacking result after poststack Kirchhoff migration, FXdeconvolution, and for display has a bandpass filter (8-12-55-70) and 1000 ms window scaling. (a) is the baseline stack, (b) is the monitor stack, and (c) is the difference. . . . . . . . . . . . . . . . .

5.26 (a) shows the NRMS error of the stacks in Figure 5.25. (b) shows a histogram of the error versus frequency and (c) shows an average of the NRMS error computed from the window of analysis (light blue box) in Figure $5.25 \ldots \ldots \ldots \ldots \ldots \ldots \ldots$

5.27 Simultaneous processing stacking result after poststack Kirchhoff migration, FX-deconvolution, and for display has a bandpass filter (8-12-55-70) and $1000 \mathrm{~ms}$ window scaling. (a) is the baseline stack, (b) is the SCMF monitor stack, and (c) is the difference. . . . . . . . . . . . .

5.28 (a) shows the NRMS error of the stacks in Figure 5.27. (b) shows a histogram of the error versus frequency and (c) shows an average of the NRMS error computed from the window of analysis (light blue box) in Figure 5.27. . . . . . . . . . . . . . . . . .

5.29 Parallel processing stacking result shown in Figure 5.25 after poststack matching of amplitudes and time-shifts. (a) is the baseline stack, (b) is the monitor stack after matching, and (c) is their difference. . . . . . .

5.30 (a) shows the NRMS error of the stacks in Figure 5.25. (b) shows a histogram of the error versus frequency and (c) shows an average of the NRMS error computed from the window of analysis (light blue box) in

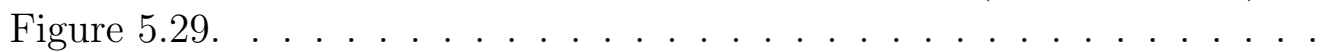

5.31 Simultaneous processing stacking result shown in Figure 5.27 after poststack matching of amplitudes and time-shifts. (a) is the baseline stack, (b) is the monitor stack after matching, and (c) is their difference. . . . . 
5.32 (a) shows the NRMS error of the stacks in Figure 5.25. (b) shows a histogram of the error versus frequency and (c) shows an average of the NRMS error computed from the window of analysis (light blue box) in Figure $5.31 \ldots \ldots \ldots \ldots \ldots \ldots$ 


\section{List of Symbols and Abbreviations}

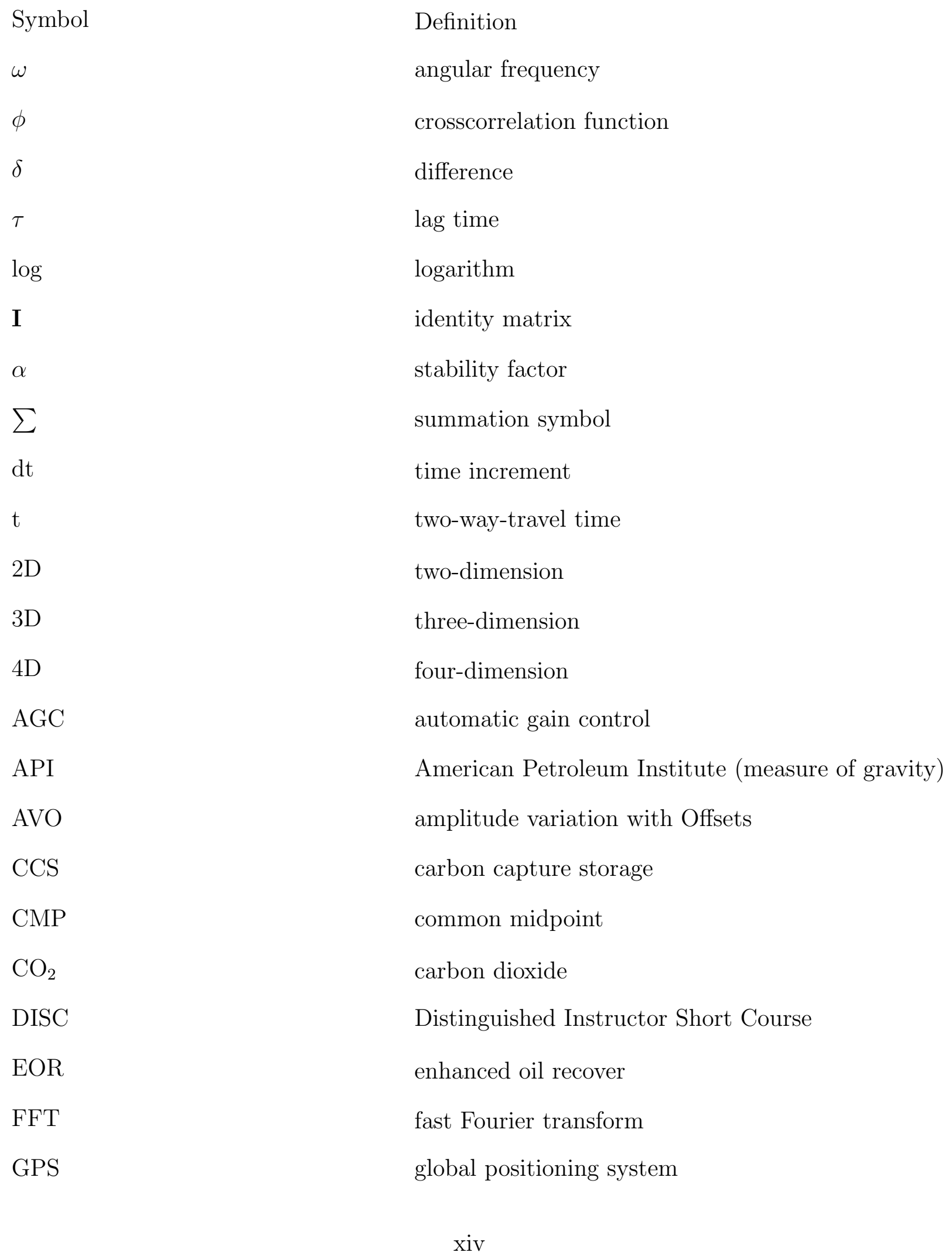




\begin{tabular}{|c|c|}
\hline $\mathrm{H}_{2} \mathrm{O}$ & water \\
\hline IFFT & inverse fast Fourier transform \\
\hline LSQ & least-squares \\
\hline $\mathrm{mD}$ & millidarcy \\
\hline Mpa & megapascal \\
\hline P-wave & compressional wave \\
\hline NMO & normal-moveout \\
\hline NRMS & normalised root mean square \\
\hline $\mathrm{OBC}$ & ocean bottom cables \\
\hline $\mathrm{OBN}$ & ocean bottom nodes \\
\hline $\mathrm{RMO}$ & residual moveout \\
\hline RMS & root mean square \\
\hline RMSD & root mean square of difference \\
\hline SVD & singular value decomposition \\
\hline SCAC & surface-consistent amplitude correction \\
\hline $\mathrm{SCMF}$ & surface-consistent matching filters \\
\hline SCSD & surface-consistent spiking deconvolution \\
\hline SEG & Society of Exploration Geophysicists \\
\hline TVD & true vertical depth \\
\hline VSP & vertical seismic profiling \\
\hline
\end{tabular}




\section{Chapter 1}

\section{Introduction}

\subsection{Reservoir monitoring}

Subsurface seismic monitoring technology has been used since the 1970's. Reservoir geophysicists repeat 2D or 3D seismic surveys to study reservoir conditions in order to optimally develop hydrocarbon fields. The goal of this monitoring is to lengthen a field's production life, prevent water invasion, and increase hydrocarbon recovery. Seismic monitoring technology simply adds the time dimension to seismic data by acquiring surveys repeatedly over the elapsed time (days, months, or years).

The effects of temperature on the elastic properties of oil-filled rocks was studied by Nur (1982) and Wang and Nur (1989) who showed the dependence of P-wave velocity on temperature at $100 \%$ oil saturation level. Because of the success of this study and the potential value seen in applying those lab measurements, seismic data was used to monitor steam injection used to enhance heavy oil recovery (Greaves and Fulp, 1987). By late 1990's, seismic monitoring technology had been reported in many published studies including Johnstad et al. (1993); Eastwood et al. (1994); Lumley et al. (1997); Beasley et al. (1997); Ross and Altan (1997). The first SEG Distinguished Instructor Short Course or DISC, "Time-lapse Seismic in Reservoir Management" was presented by Ian Jack (Jack, 1998) and focused on seismic monitoring as an emerging technology. By 2005, the second SEG DISC was presented by Calvert (2005); but since the technology had been accepted by many, the course aim was to document some of the challenges facing seismic monitoring, and possible ways of solving them, as well as other challenges that require further research. 
There are many benefits of utilizing seismic data as a monitoring tool of the subsurface (Lumley, 1995; Jack, 1998; Calvert, 2005; Barkved, 2012; Johnston, 2013), including:

1. Monitoring the spatial extent (lateral and vertical) of the fluids that are to be produced or have been injected,

2. Inferring the forces that control the fluid movement and distribution in the pore space,

3. Detecting changes in fluid contacts,

4. Detecting bypassed hydrocarbons and hence better placement of producing wells,

5. Studying compartmentalization due to faults/fractures or caused by permeability variations,

6. Observing pressure changes such as pressure depletion of a reservoir.

Today, time-lapse technology is being applied in many oil and gas fields around the world. For conventional oil and gas fields, increasing energy demand has led to new techniques for recovering larger portions of the known assets. Monitoring these subsurface assets as they are produced is important to both producing and consuming nations alike.

\subsection{Time-lapse seismic: acquisition and processing}

In this section, we briefly review some challenging aspects of acquiring and processing seismic monitoring data sets. From this review, we will highlight the issues that require mitigation and how we approach them in this thesis. We will focus on issues related primarily to onshore monitoring; however in some situations, we will present some offshore time-lapse practices for comparisons only. 


\subsubsection{Acquisition repeatability}

The quality of a seismic monitoring program is dependent on the quality of acquisition, the quality of processing, and the quality of interpretation. A well-designed time-lapse acquisition program is critical in order to obtain acquisition geometry that enables us to minimize the mismatch between repeated surveys. The survey location affects what is an acceptable tolerance between actual and planned source and receiver positions. This deviation is known as $\delta \mathrm{Src}+\delta$ Rec, referring to changes in source location and changes in receiver location, respectively (Landrø, 1999; Campbell et al., 2005). This deviation depends on near surface complexity and diffraction as well as the amplitude of the timelapse signal at the reservoir level.

In marine acquisition, towed streamers experience lateral movements due to sea currents. This lateral displacement is known as feathering. When planning for a time-lapse survey using towed streamers, this feathering must be considered carefully as the objective is to repeat the acquisition geometry as closely as possible. Recent advancement in marine acquisition has made it possible to compensate for feathering such as reshooting streamer surveys to repeat shot positions and obtain streamer swaths overlap (Calvert, 2005). Other new technology includes new acquisition tools, such as ocean bottom cables/nodes (OBC/OBN), and permanently installed receivers (Barkved, 2012). These advancements have made it possible to acquire highly repeatable seismic monitoring surveys with low error.

In land acquisition, high repeatability of acquisition geometry is possible due to the high accuracy of GPS positioning technology. However, variations of near-surface conditions can be orders of magnitude higher than the measured time-lapse difference compared to marine time-lapse surveys. Earlier work by Pullin et al. (1987) showed that acquiring seismic by burying geophones $10 \mathrm{~m}$ below the surface result in high signal-to-noise ratio data compared to seismic data recorded from surface. More recently, Schissele et al. 
(2009) and Bakulin et al. (2012) presented tests of land acquisition where sources and/or receivers are buried below the weathering zone. In the case presented by Schissele et al. (2009), the best repeatability was obtained by burying both sources and receivers below the weathering zone. The feasibility test presented by Bakulin et al. (2012) demonstrated that burying receivers below the weathering zone and using surface sources significantly improved time-lapse repeatability.

There are many other factors that are always addressed for planning a seismic timelapse survey including survey orientation, shooting direction, receiver array direction, source positions and depths, receiver positions and receiver array configuration, fold coverage, sample rate, and many others (Barkved, 2012). Some of these are applicable to marine and some only to land acquisition. But in any survey design, such elements are studied and planned carefully in order to obtain a successful and repeatable survey.

\subsubsection{Processing repeatability}

In time-lapse processing, the objective is to compensate for the nonrepeatabilty in acquisition, reduce nonrepeatable ambient noise, and enhance the time-lapse signal due to changes in fluid, pressure and stress. To achieve this objective, here are some of the main elements:

- Matching the position of source and receiver pairs in time-lapse surveys is known as 4D binning, an effective process for minimizing mismatch in positioning and improving repeatability (Campbell et al., 2005).

- Another challenge in processing time-lapse surveys is reducing nonrepeatable ambient noise. Surface equipment and installations as well as complexities in the near-surface are possible sources of these noises. Minimizing ambient noise is required when processing time-lapse data set in order to improve the match between repeated surveys. 
- Heterogeneity in the near surface and in the water column offshore, causes small seismic event timing errors that require correction.

Additional differences between time-lapse surveys are examined and if these differences are larger than the time-lapse signal of the target then correction using deterministic matching is applied. In some cases, these corrections might reduce some of the real timelapse signal or introduce a false time-lapse difference, in which case detail investigation is required (Barkved, 2012).

To address repeatability issues, there have been developments in time-lapse processing schemes, including parallel processing and simultaneous processing that were described by Lumley (2001). In the parallel approach, the data are examined independently after each processing step so that nonrepeatable differences are minimized between the two surveys. All the parameters in the parallel scheme are tested to maximize similarity between surveys. In the simultaneous processing method (Calvert, 2005; Lumley, 2001), time-lapse data are merged for many of the processing steps, where data share common statics and other common processing parameters.

Time-lapse processing has evolved over the past decade; however several challenges remain. Some of these challenges are the mismatch caused by the time-dependent changes in the near surface between surveys. The near surface acts as a filter that affects the traveltime, amplitude and the shape of the propagating waves. These can have dramatic effects on the true subsurface seismic response caused by fluid production or injection. Therefore, to correctly process the time-lapse data set, a process known as seismic crossequalization (Ross et al., 1996) can be applied pre and/or post-stack to attenuate the unwanted near-surface effects on the source waveform, the receiver response, the statics, and the uncorrelated ambient noise. 


\subsubsection{Remaining challenges}

In an ideal time-lapse experiment, seismic image differences should only be present where there is fluid production or injection or a pressure change. Figure 1.1 is a simple earth model demonstrating change in a thin layer at a depth of about $750 \mathrm{~m}$. Since the nearsurface conditions are unchanged between the baseline and the monitor survey, and a perfectly repeated acquisition geometry for the two surveys, the time-lapse difference (Figure 1.2) shows a clear seismic response due to changes at the reservoir level.

However, complex near surface conditions, such as seasonal fluctuations in the water table or even daily variations in temperature, can have a significant effect on time-lapse surveys. Figure 1.3 shows the same model presented in Figure 1.1 but with lateral variations of near surface velocity within the same model and between the baseline and the monitor models. This change in the near-surface velocity causes traveltime difference between the baseline and the monitor events (Figure 1.4). Note that in the difference section, the time-lapse response to reservoir change is more difficult to see.

In this dissertation, we show that the near-surface effects seen above can be diminished using information recorded in the time-lapse data. As we will see in Chapters 2 and 3 , the seismic trace can be modeled as the convolution of four terms which are named: the source, the receiver, the offset, and the midpoint terms after the spatial coordinate upon which they depend. The first two terms are surface-consistent, meaning they capture much of the near-surface geology effects. The last two terms are subsurface-consistent, meaning they respond more to subsurface geology. As described above, most of the unwanted seismic response differences are associated with the near surface, and they can mask small seismic response differences due to changes in the subsurface. Therefore, estimating these near-surface-related variations from the available seismic data, and then incorporating them as filters to minimize the difference between repeated surveys will be the topic of Chapter 3. Moreover, after applying these new matching filters, simul- 


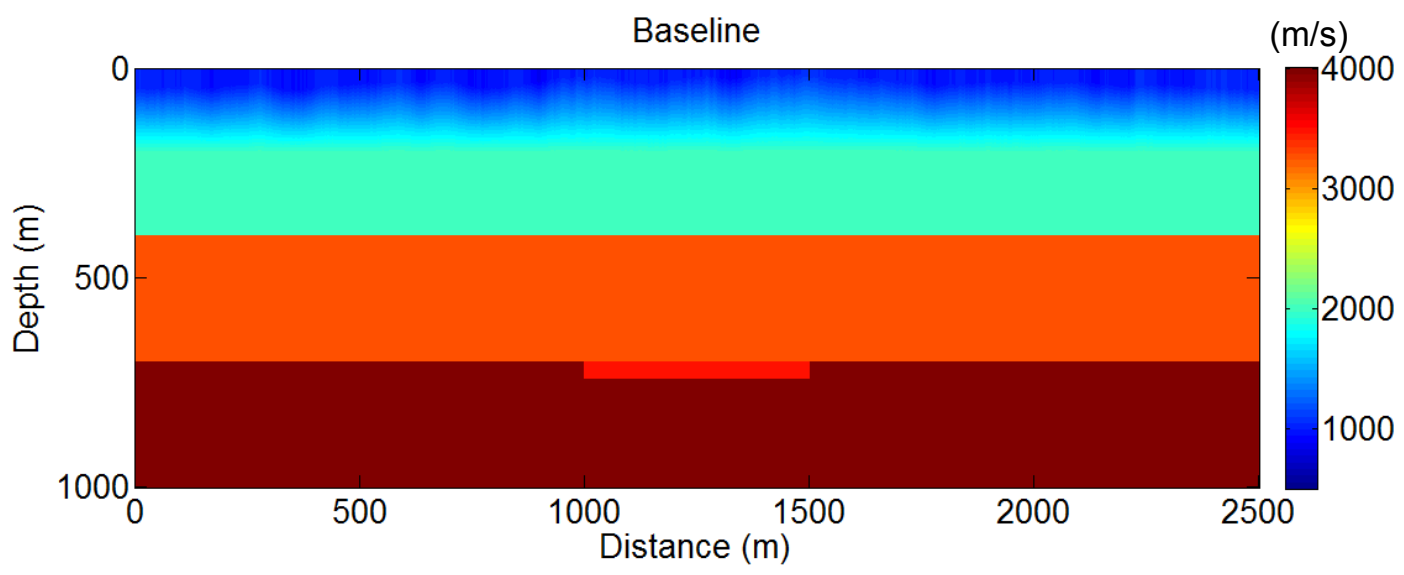

(a)

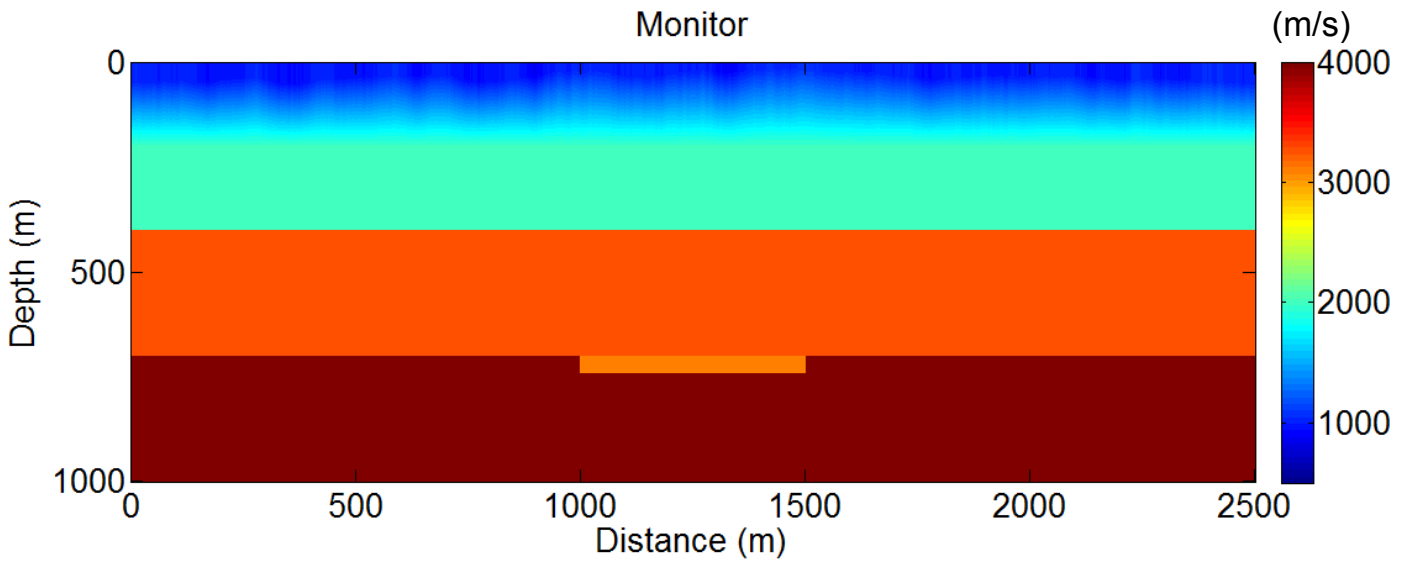

(b)

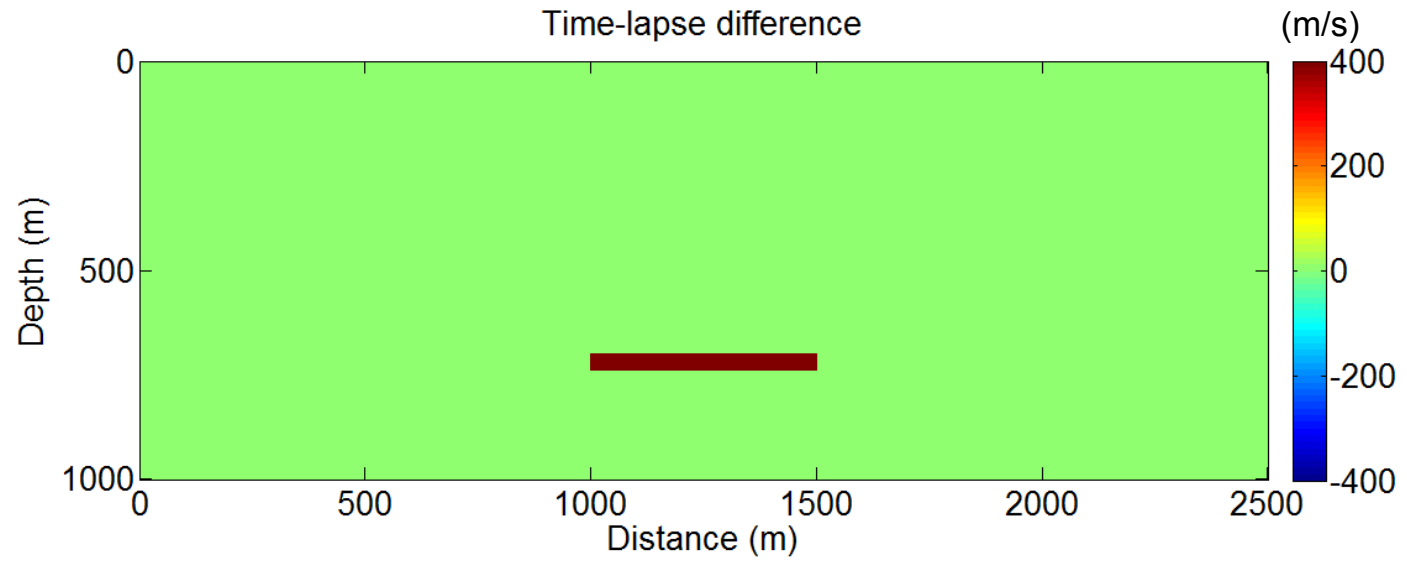

(c)

Figure 1.1: Two simple earth models $(\mathrm{m} / \mathrm{s})$ representing baseline $(\mathrm{a})$, monitor $(\mathrm{b})$, and their time-lapse difference (c). The only difference is in the reservoir slab depicting a subsurface change due to reservoir condition change. 


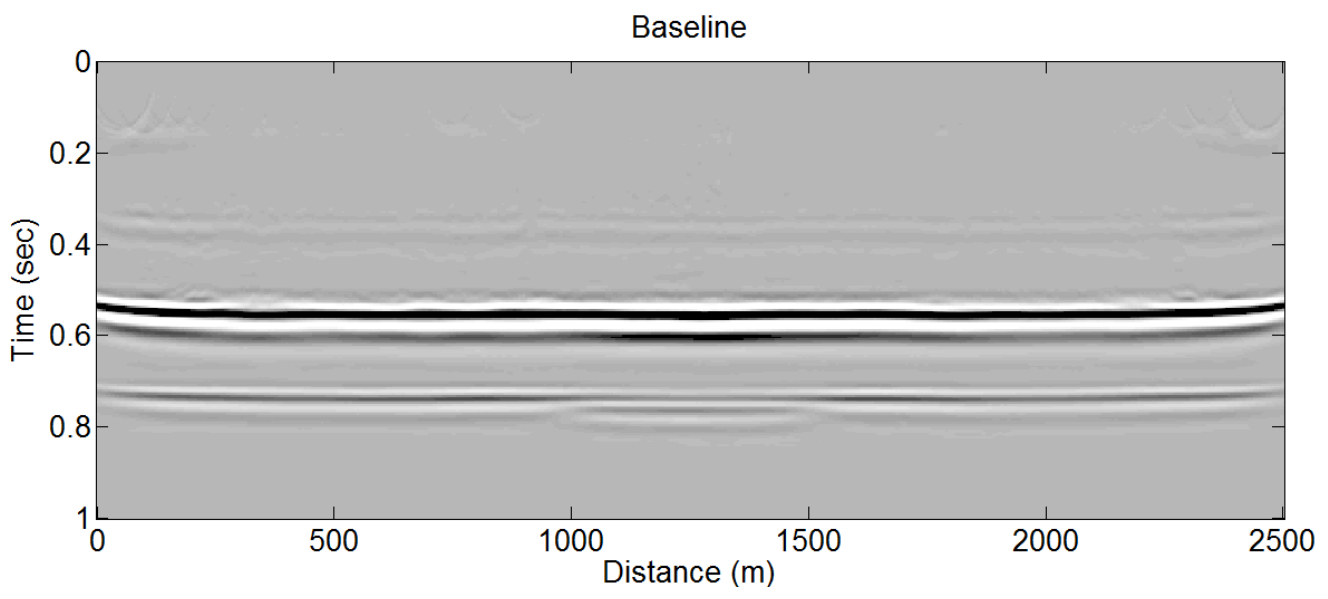

(a)

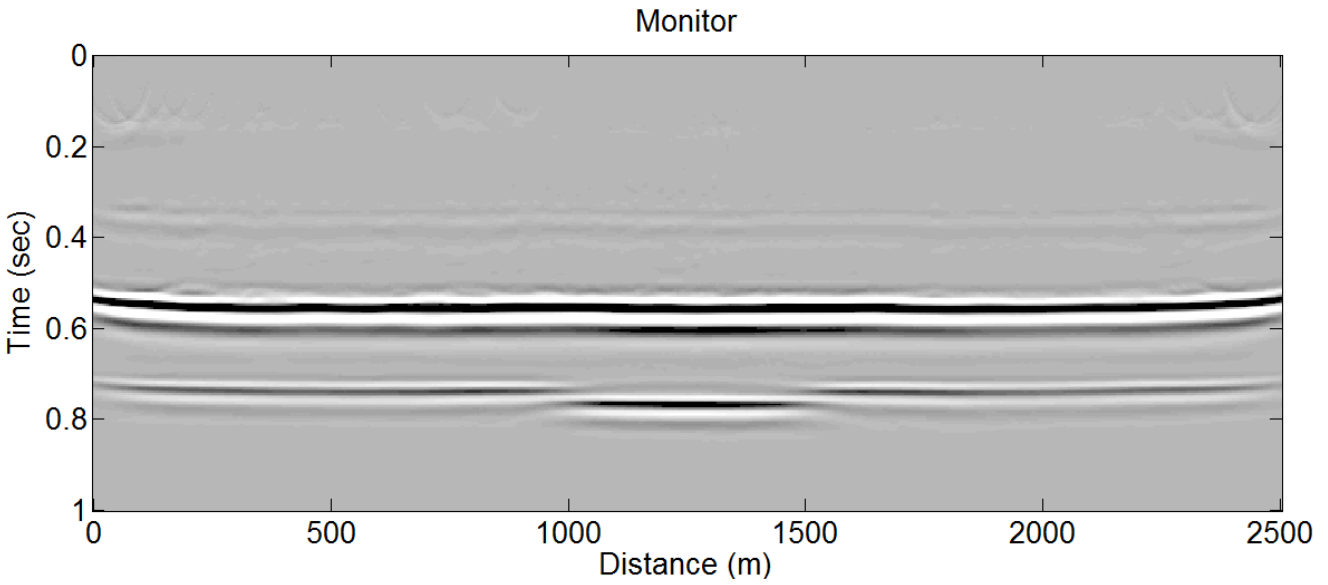

(b)

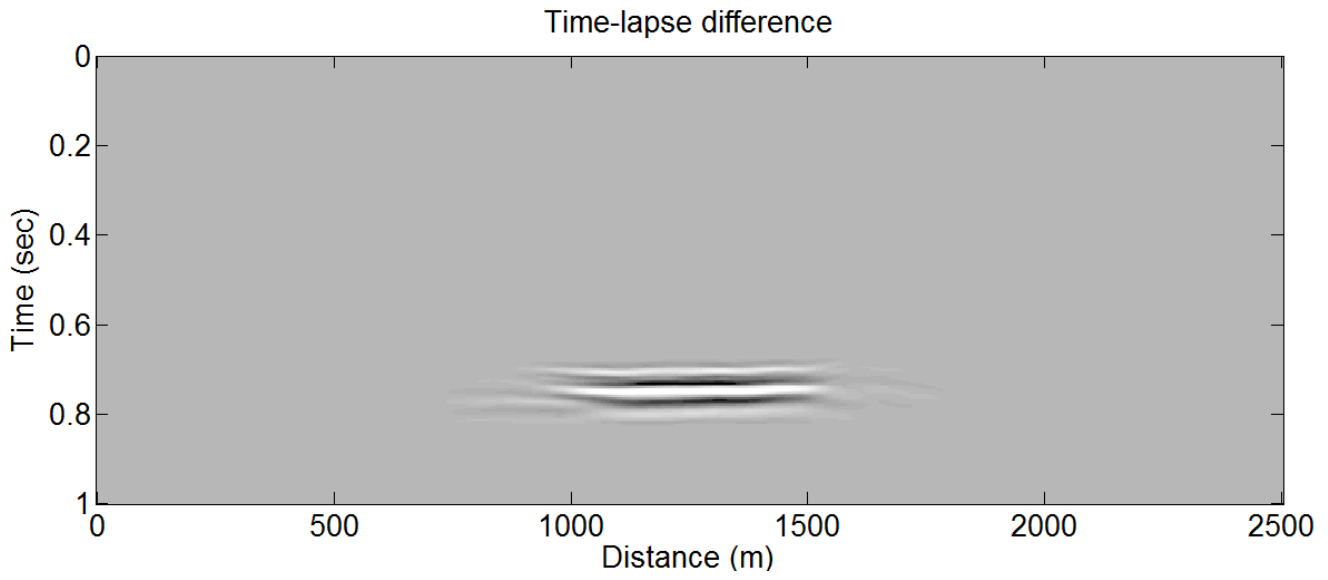

(c)

Figure 1.2: Synthetic seismic data corresponding to the velocity model in Figure 1.1. (a) is the baseline seismic, (b) is the monitor seismic (same amplitude scaling as baseline), and their time-lapse difference (scaled by 5) is in (c). Note the difference is only at the reservoir level as expected. 


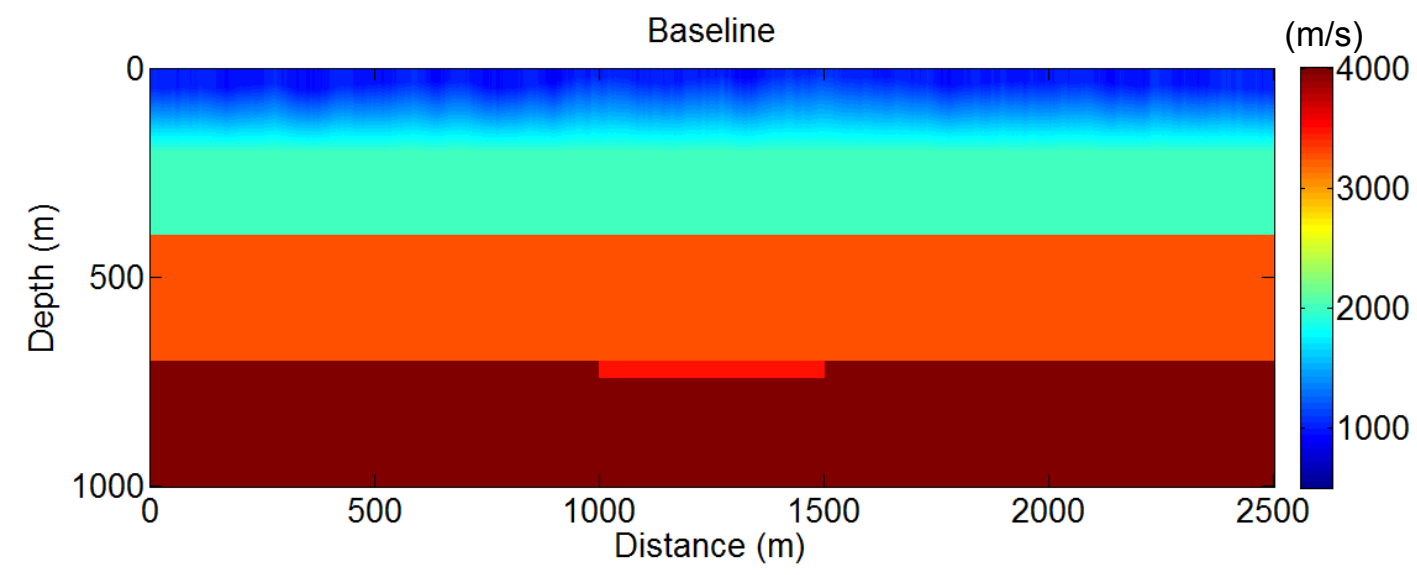

(a)

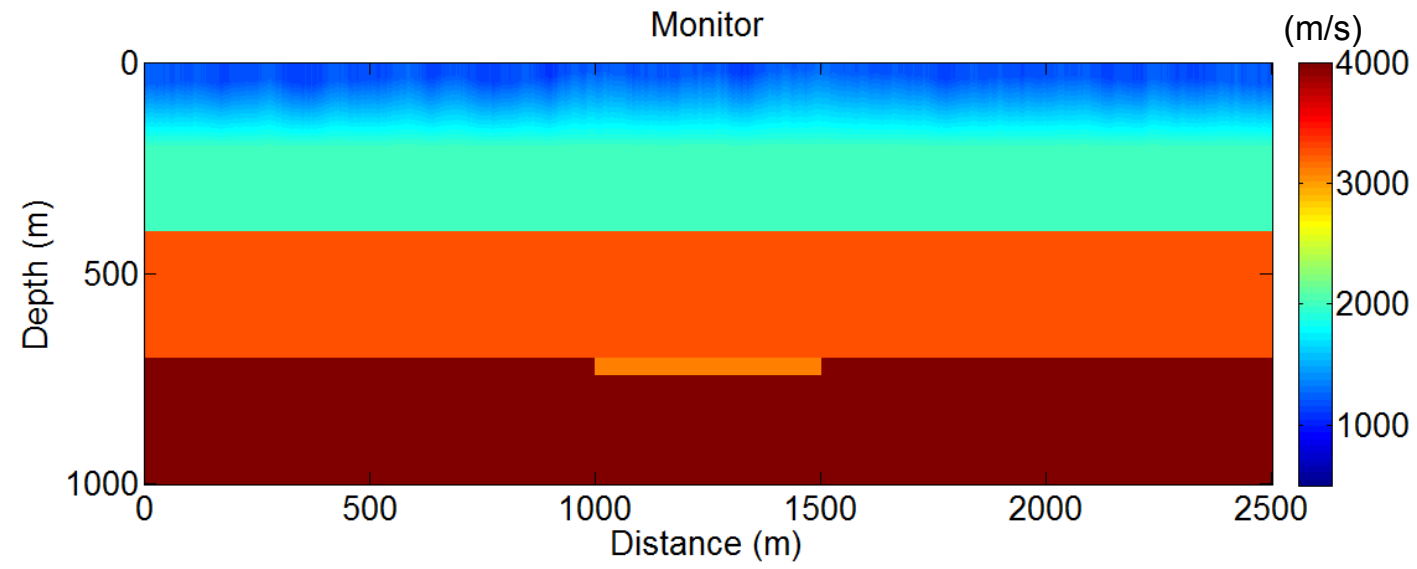

(b)

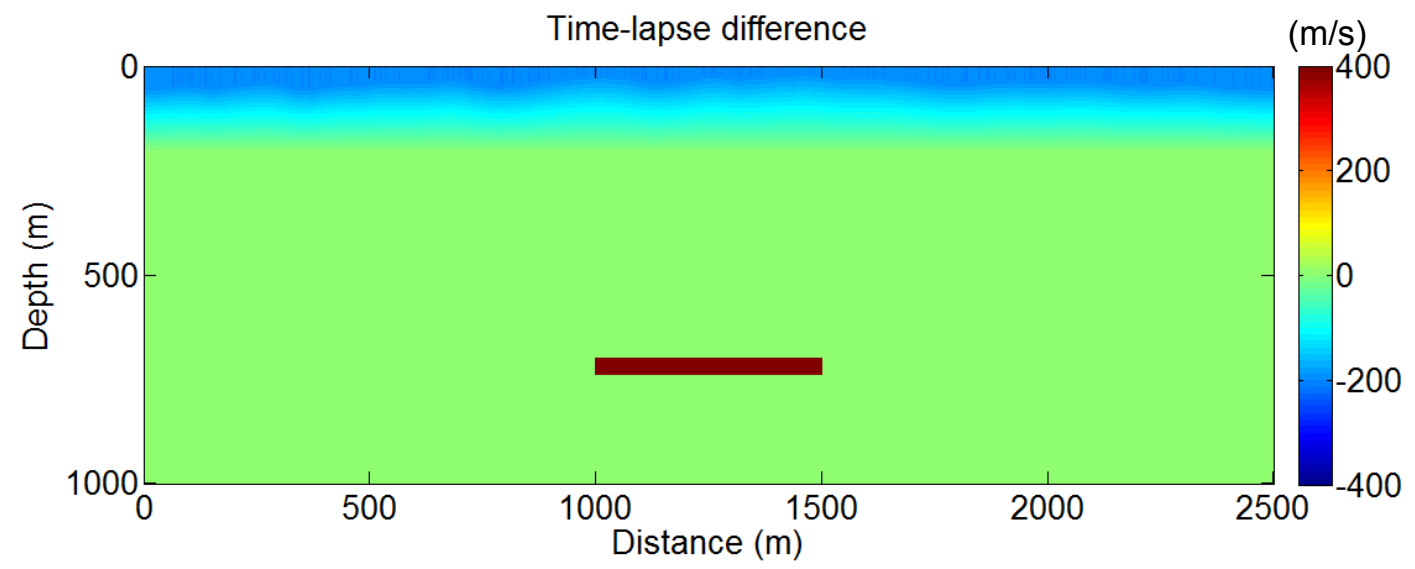

(c)

Figure 1.3: Two earth velocity models (units in $\mathrm{m} / \mathrm{s}$ ) representing baseline (a), monitor (b), and their time-lapse difference (c). Note that both models have both near surface and subsurface variations depicting a real land time-lapse acquisition. 


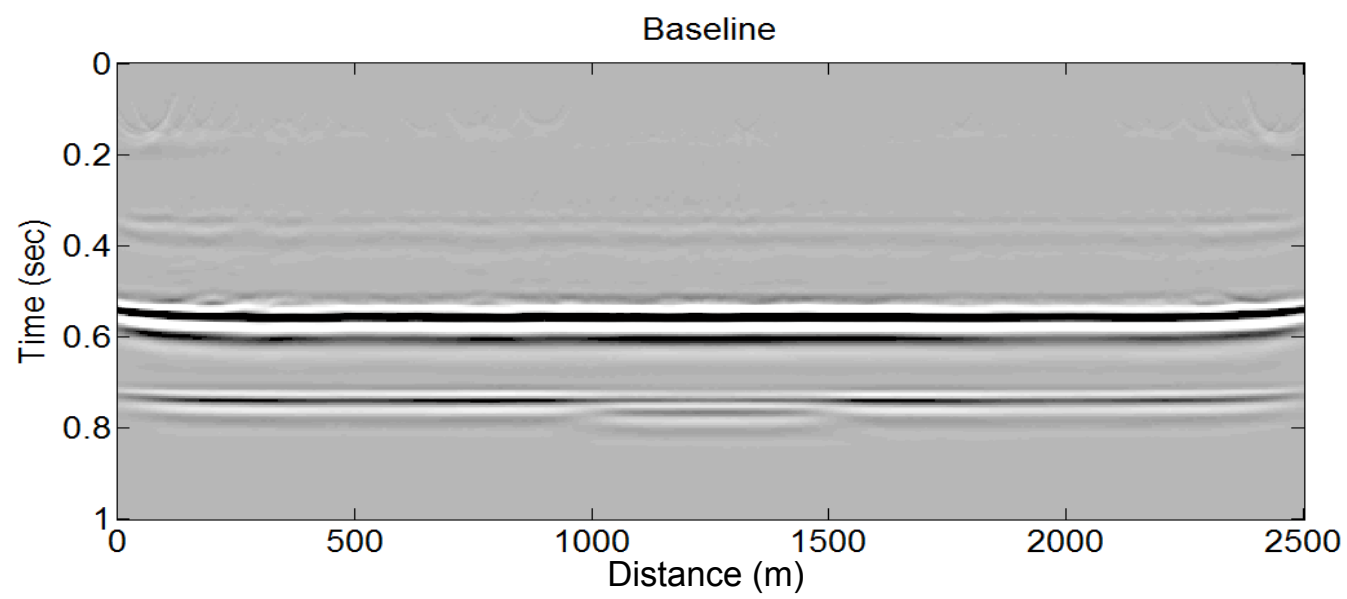

(a)

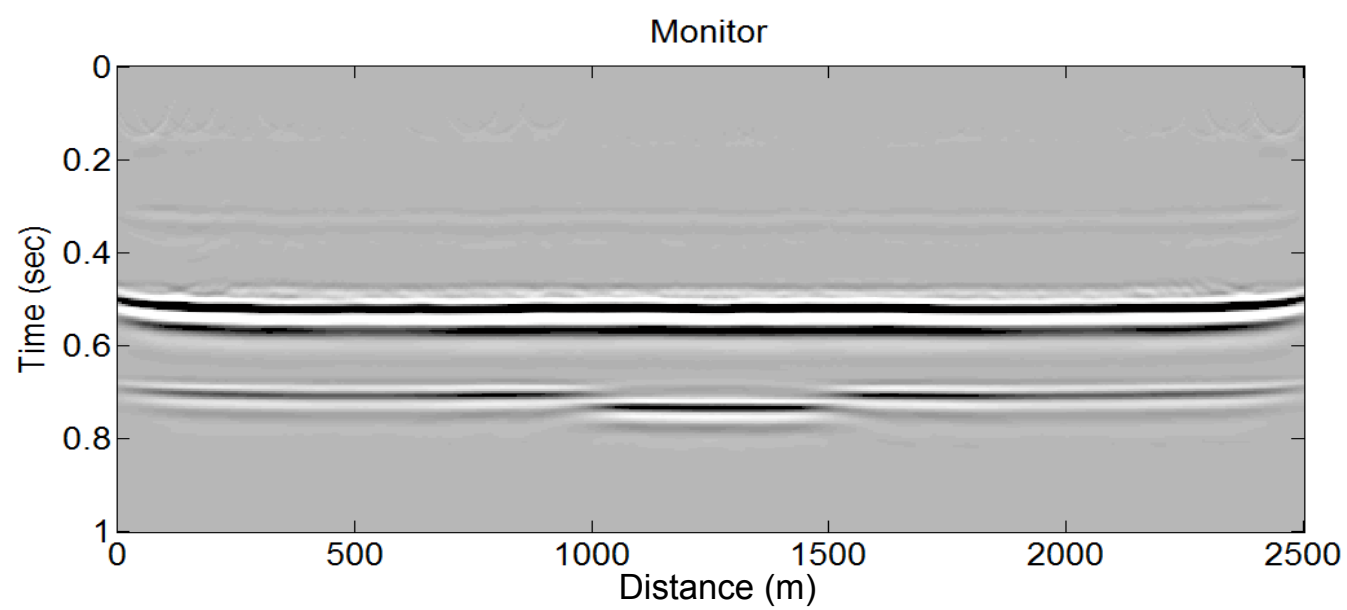

(b)

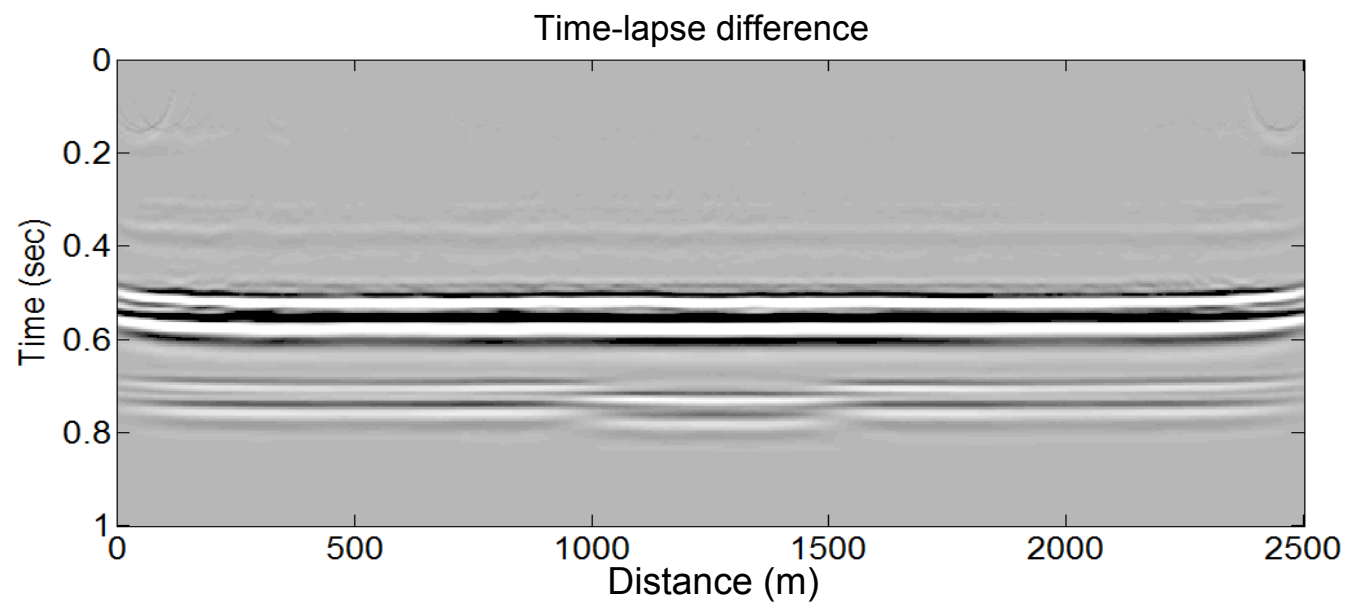

(c)

Figure 1.4: Synthetic seismic data corresponding to the velocity model in Figure 1.3. (a) is the baseline seismic, (b) is the monitor seismic, and their time-lapse difference is in (c). All plots have the same amplitude scaling as the baseline plot. 
taneous processing, described above, is presented here with some modifications. These modifications will be explained using examples in Chapters 4 and 5 .

\subsection{Dissertation contributions}

The main contributions of this dissertation can be summarized as follows:

1. Theoretical extension of the surface-consistent model so that the data term is composed of the ratio of two data sets instead of only one;

2. Formulation of a surface-consistent matching filter (SCMF) based on two concepts: the surface-consistent model and the matching filter. The new SCMF is designed to match one dataset to another in a surface-consistent manner. The SCMF improves repeatability, which is particularly an issue for land seismic monitoring programs. This new algorithm corrects for time-shifts, amplitude difference, and phase difference, employing the surface-consistent model.

3. Solution of the matching filter with time-domain least-squares algorithm, demonstrating the accuracy and the stability of the time-domain solution when compared to the spectral ratio method.

4. Modification of the simultaneous processing scheme generally advocated for time-lapse processing, such that there are four-components common between any two surveys instead of only midpoint and offset terms. This modification is possible after applying the SCMF algorithm. 


\subsection{Dissertation organization}

Our primary objective in this dissertation is to focus on the new algorithm outlined in Section 3.2, and how the modification can be used to solve a known geophysical problem; primarily repeatability issues (i.e time shifts, amplitude differences, and phase shifts) in time-lapse seismic studies. Our secondary objective is to examine existing timelapse processing methods, such as that mentioned in Section 1.3, and explore possible adaptation of these methods in order to maximize the benefits of the new algorithm.

In Chapter 2, we review the surface-consistent model, its theory, assumption, and some of its applications in exploration geophysics. In addition, we will touch briefly on the reliability of the surface-consistent methods and ways to measure the accuracy in the context of time-lapse seismic.

In Chapter 3, a new extension of the surface-consistent model will be formulated. Its relation to a well-known algorithm, mainly match filtering and surface-consistent model, will be outlined. We will also explore ways to solve the new system of equations and demonstrate that with examples.

In Chapter 4, through a synthetic time-lapse example, we will discuss the details of how to compute and apply the new algorithm. One time-lapse processing scheme will be discussed, that is the simultaneous processing after surface-consistent matching filters.

In Chapter $5, \mathrm{a} \mathrm{CO}_{2}$ monitoring example will be presented. Details of two time-lapse processing schemes will be compared. The first one is called independent processing and the second is known as simultaneous processing. The first scheme will be a generic processing flow generally applied in time-lapse processing. The second scheme has been modified in this dissertation from the well known simultaneous time-lapse processing discussed in literature such as Lumley et al. (2003) and Calvert (2005).

In Chapter 6, we present the conclusions of this dissertation. 


\section{Chapter 2}

\section{The surface-consistent model}

\subsection{Introduction}

The concept of surface-consistency has been used since the early days by seismic explorationists specifically for solving statics due to changes in elevation and to variations in near-surface velocity. There were many techniques attempting to formulate and estimate these time anomalies in order to produce a plausible seismic section. The work by Hileman et al. (1968) on automatic static corrections, and many others during the 1960's, described several ways of computing statics. Schneider (1971) and Taner et al. (1974) were first to generalize and formulate all previous attempts into a new model that became known as the "surface-consistent model". This basic model had some assumptions and a basic general equation to be described later. Due to its simplicity and effectiveness, the surface-consistent model became one of the most widely used models in seismic data processing techniques, particularly for statics, deconvolution, and amplitude and phase corrections.

In this chapter, we review the surface-consistent model, its basic assumptions, and its general equation. A discussion of the main application in seismic data processing is also presented.

\subsection{The surface-consistent hypothesis}

Conceptually, the earth is considered to be separable into a thin near-surface layer, subject to weathering effects and other seasonal changes, and the deeper subsurface where such changes are largely absent. The near-surface layer is considered to be a filter 
that modulates downgoing and upgoing waves traveling through it. Subsurface effects are more likely to be nonstationary (varying with depth) but near-surface effects can be approximated by a filter in a suitably restricted time zone.

\subsubsection{The surface diagram}

Prior to discussing the concept of the surface-consistent model, it is important to review the coordinate system on which this model is based. This coordinate system is known as the "surface diagram" used by Morgan (1970), Shah (1973), Taner et al. (1974) and Taner and Koehler (1981). It identifies each trace with its source and receiver coordinates with respect to a reference coordinate system, as well as offset and midpoint coordinates. An offset is defined as the source-to-receiver distance and the midpoint is defined as the point on the surface halfway between the source and the receiver. The four principal trace coordinate directions in this diagram (Figure 2.1) are:

1. Common source direction where source number is fixed but receiver number varies.

2. Common receiver direction where source number varies but receiver number is fixed; orthogonal to common-source direction

3. Common offset direction at an angle of 45 degrees to both common-source and common-receiver planes.

4. Common midpoint (CMP) direction at an angle of 45 degrees to the commonsource or common-receiver axis and orthogonal to the common offset direction.

The zero-offset plane is where source and receiver numbers coincide, and traces are projected to this plane for CMP-stacking. 


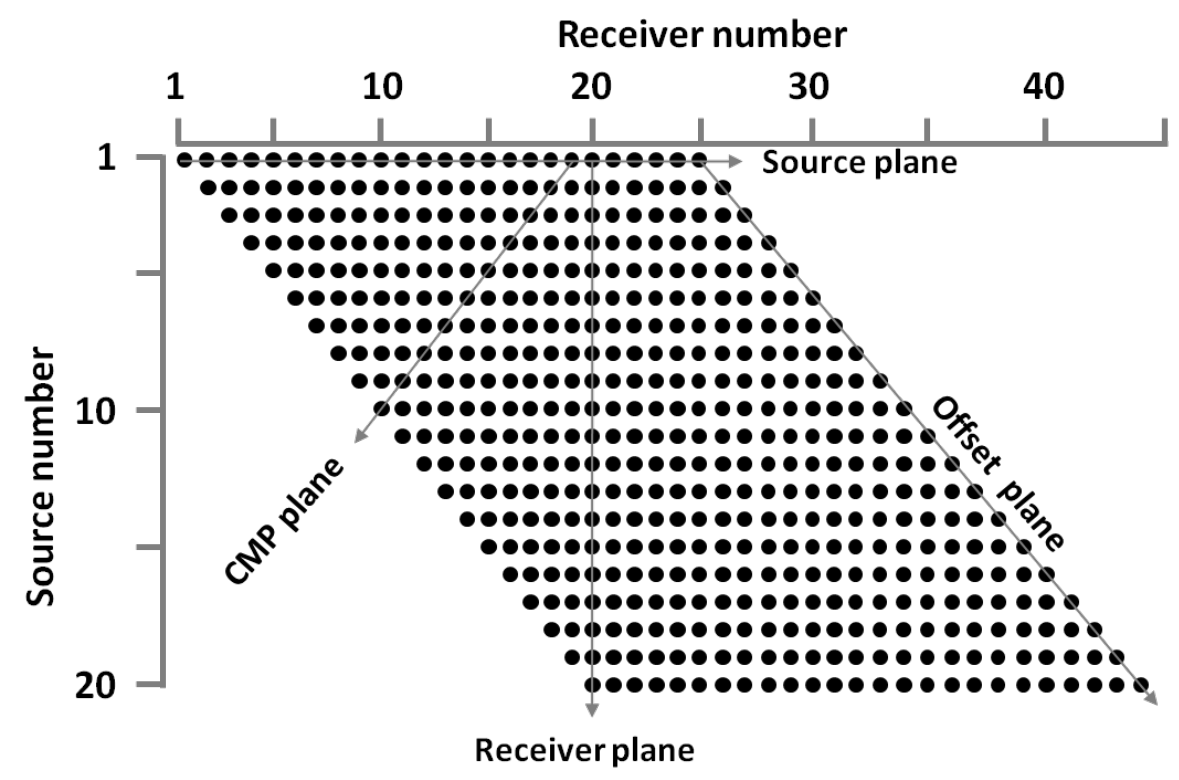

Figure 2.1: Surface diagram and principal trace organization.

\subsubsection{Basic assumptions}

Taner and Koehler (1981) made four assumptions allowing them to approximate the surface-consistent corrections required to compensate the previously mentioned nearsurface effects. Those assumptions were:

1. The near-surface related factors are the same throughout the seismic recording time (i.e source response and coupling, near-surface attenuation beneath each source or receiver, and receiver response and coupling).

2. Each source has a characteristic strength and signature (i.e. waveform) that will affect all traces recorded from that source and similarly the receiver coupling effect applies to all traces recorded at a specific receiver from any source.

3. All traces contributing to a particular midpoint gather location contain the same subsurface information with expected offset variation. 
4. All traces must be corrected for spherical divergence, normal moveout (NMO), and field statics in order to eliminate any amplitude or arrival time corrections within a time window for a CMP gather.

Spherical divergence is defined as a decay of the wave amplitude as it propagates away from a seismic source. In a homogenous medium, this decay is proportional to $1 / \mathrm{r}$ where $\mathrm{r}$ is the radius of the wavefront. The normal moveout is a correction that compensates for the delay in the arrival times of a flat reflector at depth due to the offset between seismic sources and receivers. The trajectory of these arrival times as a function of offset follows a hyperbola, and NMO removes the moveout effect on traveltimes.

Conceptually, the model separates near-surface effects from subsurface effects through the dependence of the data upon the acquisition coordinates. Variations in the data that depend strongly on the source or receiver coordinates are assumed to arise from features in the very near-surface. Alternatively, data variations that depend more strongly on midpoint and offset coordinates are attributed to features in the deeper subsurface.

\subsubsection{General equation}

Taner et al. (1974) and Taner and Koehler (1981) represented the trace as the convolution of four terms expressed by:

$$
d_{i j}(t) \approx \underbrace{s_{i}(t) * r_{j}(t)}_{\text {Near-surface }} * \underbrace{h_{k}(t) * y_{l}(t)}_{\text {Subsurface }},
$$

where "*" denotes convolution in the time domain, $t$, and

- $d_{i j}(t)$ : Seismic trace resulting from the $i^{\text {th }}$ source recorded into the $j^{\text {th }}$ receiver.

- $s_{i}(t)$ : Source response at surface location $i$.

- $r_{j}(t)$ : Receiver response at surface location $j$. 
- $h_{k}(t)$ : Offset response at offset location $k$.

- $y_{l}(t)$ : Midpoint response below surface location $l$.

The $s_{i}(t)$ term includes such things as the source strength and waveform, and any attenuation or ghosting near the source location. The $r_{j}(t)$ term contains the receiver coupling strength and the influence of geology near the receiver on the recorded waveform. The offset term contains subsurface effects that are offset dependent, such as spherical divergence, or residual moveout, or surface waves, or AVO. The last term, midpoint, captures the subsurface response below surface location $l$ and is usually considered to be proportional to normal-incidence reflectivity. This contains the response of all traces with common midpoint and may include multiples and attenuation along the raypath. Offset location $k$ is computed as $k=|i-j|$, and midpoint location is $l=\frac{1}{2}(i+j)$ (technically midpoint locations define a regular grid with half the spacing of the receiver grid).

In equation 2.1, the first two terms are source consistent and receiver consistent and describe effects attributable to the near-surface. The second two terms are offset consistent and midpoint consistent and describe effects attributable to the subsurface.

Equation 2.1 is based on acquisition geometry and is not "derived" from any wavetheoretical concepts. Consequently, even the best noise-free data will never be described exactly by this equation. For example, the surface-consistent model is manifestly stationary (convolution is a stationary operation) and nonstationary effects such as moveout and anelastic attenuation can only be modeled approximately by restricting the analysis to small time windows. The introduction of the offset term and the midpoint term by Taner and Coburn (1980) and Taner and Koehler (1981) improved the fit to the data. Artifacts due to stretching and muting are attributed to offset terms (Taner and Koehler, 1981). Midpoint terms are assumed to be the normal incidence reflectivity where all traces at the same midpoint location contain the same subsurface information (Taner 
and Koehler, 1981). This normal incidence process is well approximated by applying corrections such as field statics (land data), spherical divergence, and normal moveout (Taner and Koehler, 1981; Claerbout, 1986; Cambois and Stoffa, 1992). In summary, equation 2.1 is an approximate trace model but with a long history of successful applications.

\subsection{Applications in seismic data processing}

We describe below two of the main applications of the surface-consistent model in seismic data processing, that is residual static corrections and deconvolution. The accuracy of the model is briefly discussed using a simple synthetic example.

\subsubsection{Residual static corrections}

Residual static corrections are a subset of the static corrections designed to correct for small error in the near-surface model. Sheriff (2002) defined statics as

"corrections applied to seismic data to compensate for the effects of variations in elevation, weathering thickness, weathering velocity, or reference to a datum. The objective is to determine the reflection arrival times which would have been observed if all measurements had been made on a (usually) flat plane with no weathering or low-velocity material present."

Chapter 3 in Cox (1999) discusses the details of how to compute datum statics (also known as field statics as they can be computed by field crews). They are the time shifts needed at shot and receiver locations to correct traces to appear as though they were collected with the shots and receivers at a new datum level. The basic concept of static corrections is illustrated in Figure 2.2 which shows a schematic diagram depicting the earth and a ray path from a source to a receiver. Originally, we record the data with the source $\mathrm{S}$ and receiver $\mathrm{R}$ on the surface of the earth. As shown in Figure 2.2, static- 


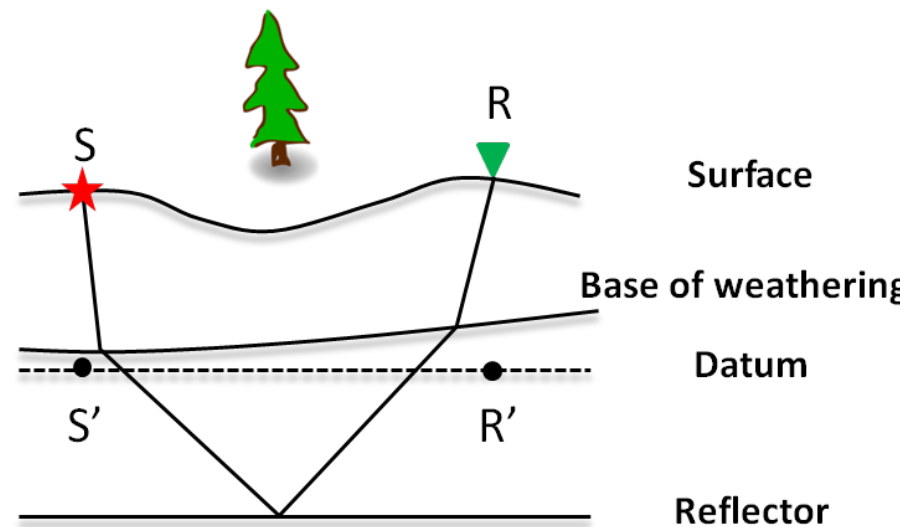

(a)

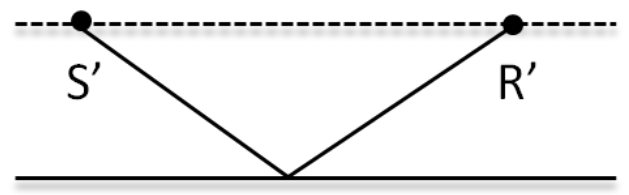

(b)

Figure 2.2: Schematic diagram depicting the earth and a ray path from a source to a receiver showing the concept of static corrections. (a) The original recorded data with the source $(\mathrm{S})$ and receiver $(\mathrm{R})$ on the surface of the earth. (b) The concept of static corrections with a pseudo datum plane below the weathering zone and a new source ( $\left.\mathrm{S}^{\prime}\right)$ and a new receiver $\left(R^{\prime}\right)$.

corrections implies that the original model is replaced by another one where a reference datum is chosen below the weathering zone and the source and receiver locations are now shifted vertically below the original surface locations, S' and R' respectively. The weathering layer often has very low velocity and a strong refractor at its base. Rays traveling in this layer tend to be vertical (this may not be true in a high-velocity layer like the permafrost). The term static implies that it is a constant time shift for the entire trace. Sheriff (2002) described this simple technique as follow:

"(d) underlying the concept of static-corrections is the assumption that a simple time shift of an entire seismic trace will yield the seismic record which would have been observed if the geophones had been displaced vertically downward to the reference datum, an assumption not strictly true."

Why is this assumption not strictly true? It is primarily due to the assumption of vertical raypaths (Figure 2.2) when computing statics. This assumption is usually not strictly true except perhaps for zero offset. However, if the weathering layer has a low 
enough velocity, then it is a good assumption. The near-surface model is an approximate one since it is generated from data with sparse control points, i.e deep upholes. The lack of detailed near-surface model information results in some inaccuracies in the model and therefore in the resulting datum static corrections. Therefore, datum statics are not a unique set of solutions since a change in reference datum results in a different set of corrections (Cox, 1999). Further measures are taken to correct or minimize the remaining errors, and these remaining errors are referred to as residual static corrections.

It is important to know that residual statics are not intended to replace a good set of datum static solutions which are generally considered as a first good approximation. A definition from Sheriff (2002) which is more specific for residual statics is that: "(c) data smoothing statics methods assume that patterns of irregularity that most events have in common result from near-surface variations and hence static-correction trace shifts should be such as to minimize such irregularities. Most automatic statics-determination programs employ statistical methods to achieve the minimization."

As a result, correcting these irregularities, as stated in the definition above, should lead to an improved final seismic section compared to a result with only datum statics (Figure 2.3).

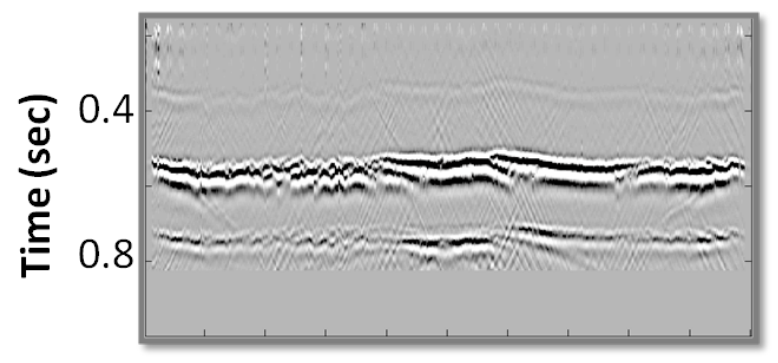

(a)

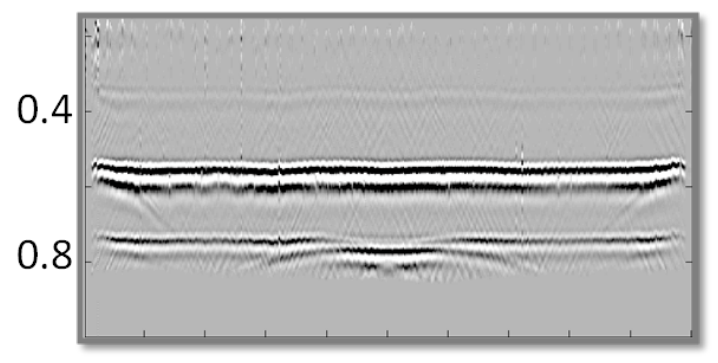

(b)

Figure 2.3: Common-midpoint stacked sections showing the seismic data with (a) datum statics only and the same one with (b) datum statics and residual static corrections applied.

Various residual static correction techniques have been developed since the early 
1970's, including, but not limited to, common-surface location, common-midpoint based, and common offset based methods, as well as the stack power approach and other statistical methods (Cox, 1999). The CMP-based method is widely used in most commercial processing softwares. The early work of Taner et al. (1974) and Wiggins et al. (1976), and others, on correcting time anomalies related to near-surface effects led to the concept of surface-consistent static corrections which are simple time shifts. Correcting for these static anomalies can have large effects on all subsequent processing steps as well as on the display of the seismic section. These time shifts distort the seismic image of a CMP stack and can cause problems with velocity picking.

From the surface-consistent model assumptions (Subsection 2.2.2), the near-surface effects do not vary with depth, and if statics are assumed surface-consistent, then they are stationary (constant with time). The goal for computing statics is to align seismic reflections, hence only relative time shifts are required. To measure these relative time differences between traces recorded from different surface positions (different source and/or receiver locations), we assume the following:

- that traces have been corrected for field statics; and

- traces must be corrected for spherical divergence and normal moveout (the latter is critical for eliminating apparent lateral velocity changes related to near-surface effects as well as for stability in statics solutions when laterally averaging residual normal moveout terms).

Basically, source and receiver coordinates are orthogonal, and thus linearly independent; but offset is linearly dependent upon both source and receiver coordinates. Therefore, any time shifts related to moveout leaks into both source and receiver statics, hence moveout correction is required.

It is very common to stack the data prior to residual statics. The stacking process is 
called initial stacking or brute stacking. An initial stack routinely includes datum statics and is used to determine whether residual statics are required as well as the choice of the time gate to use for crosscorrelation. In addition, velocity analysis before and after residual static corrections is a normal routine (Yilmaz, 2001; Cox, 1999).

To obtain the relative reflection time difference between two traces, a crosscorrelation technique is generally used. Given two traces, $d_{1}(t)$ and $d_{2}(t)$, the crosscorrelation function is:

$$
\phi(\tau)=\sum_{t=t_{1}}^{t_{2}} d_{1}(t) d_{2}(t+\tau) d t
$$

where $\phi(\tau)$ is the crosscorrelation function between the two traces, $\tau$ is the lag time, and $t_{1}$ and $t_{2}$ are the data start and end times. The time shift between two traces is obtained from the lag time at which the crosscorrelation function, $\phi(\tau)$, has a maximum peak value. The length of the input trace should be at least $t_{2}-t_{1}+\tau_{\max }$, or the time gate plus the maximum lag time, in order to avoid crosscorrelation of live traces against zeros (Cox, 1999). In addition to time gates, bandpass filtering of the data, as part of data conditioning before residual statics, often helps to estimate a time shift value that matches the maximum crosscorrelation value.

There is also the choice of the pilot or reference trace for crosscorrelation. The simplest method involves selecting a trace from a CMP gather with high signal-to-noise ratio or summing all traces in that particular CMP gather to form a reference trace. This reference trace is then used to compute the relative time shifts by crosscorrelating it with all traces in all CMP gathers.

Once the relative time shifts (relative to the reference trace) have been computed for every trace, we model them as the sum of the four terms (Schneider, 1971; Taner et al., 
1974; Wiggins et al., 1976):

$$
T_{i j} \approx \underbrace{S_{i}+R_{j}}_{\text {Near-surface }}+\underbrace{Y_{l}+M_{l} X_{i j}^{2}}_{\text {Subsurface }},
$$

where $T_{i j}$ is the relative time shift for the $i j^{t h}$ trace after the application of normal moveout (NMO) correction; $S_{i}$ is the surface-consistent source static correction (includes datum and residual terms) at surface location $i ; R_{j}$ is the surface-consistent receiver static correction (includes datum and residual terms) at surface location $j ; Y_{l}$ is the normal incidence two-way traveltime from the datum plane to subsurface reflector at the $l^{\text {th }}$ CMP location; $M_{l} X_{i j}{ }^{2}$ is the residual moveout. It accounts for the NMO errors within a specific time gate. The coefficient $M_{l}$ has the dimensions of time/distance ${ }^{2}$, and $X_{i j}$ is the offset between the $i^{\text {th }}$ source and the $j^{\text {th }}$ receiver. Equation 2.3 is a simpler form than that presented by Larner et al. (1979) as it does not include the cross-dip components or the noise component. The term surface-consistent in the above equation implies that the time correction depends on the surface location of the source and receiver associated with the trace.

Equation 2.3 is the basic traveltime form which relates the relative time shift $T_{i j}$ to the source static correction $\left(S_{i}\right)$, receiver static correction $\left(R_{j}\right)$, the structural (or CMP) time $\left(Y_{l}\right)$, and the residual moveout $\left(M_{l}\right)$. For every trace on the seismic line, we will have one equation similar to that in equation 2.3. The parameters $S_{i}, R_{j}, Y_{l}, M_{l}$ are unknowns which, when computed, form the solution of the statics problem.

Wiggins et al. (1976) summarized the residual statics problem as follows:

The linear simultaneous equations that define the statics problem are said to be overspecified (there are more equations than unknowns) and underconstrained (they are deficient in the number of independent equations available to solve for the unknowns).

Averaging the residual moveout component or smoothing the structural term are some of the constraints placed on the solution to limit the dependency of the equations on one 
another (Cox, 1999).

Wiggins et al. (1976) solved the decomposition process of equation 2.3 using the general linear inverse method. For a noise-free case, the form is:

$$
\mathrm{Gx}=\mathbf{t}
$$

where $\mathbf{t}$ is a vector of the observed time shifts, $\mathbf{x}$ is a vector of unknowns, and $\mathbf{G}$ is a coefficient matrix that depends on the field recording geometries. $\mathbf{G}$ is a sparse matrix with dimensions $m \times\left(N_{s}+N_{r}+N_{l}+N_{l}\right)$, where $m$ equals the number of traces. The residual statics problem has $N_{s}$ shot locations, $N_{r}$ receiver locations, $N_{l}$ midpoint locations, and $N_{l}$ residual moveout terms. The dimension of $\mathbf{x}$ is $\left(N_{s}+N_{r}+N_{l}+N_{l}\right)$

$\times 1$. Let's suppose a simple $2 \mathrm{D}$ example (Figure 2.4) where we have 10 shots with 10 receivers for every shot. The total number of traces is 100 . The number of midpoints is 10 , and the number of unknowns is $10+10+10+10=40$, whereas the total number of equations is 100. This simple example demonstrates the general problem highlighted by Wiggins et al. (1976) that in most seismic data acquisition, the number of traces exceeds the number of unknowns. Figure 2.5 illustrates the geometry matrix, G, for the simple example mentioned above.

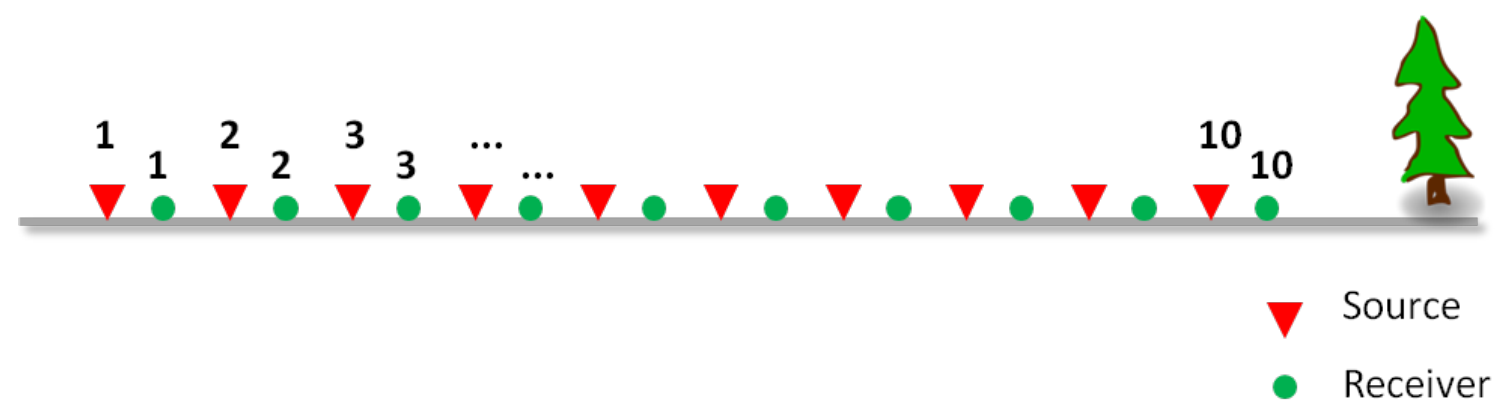

Figure 2.4: An example of 2D seismic acquisition layout. 


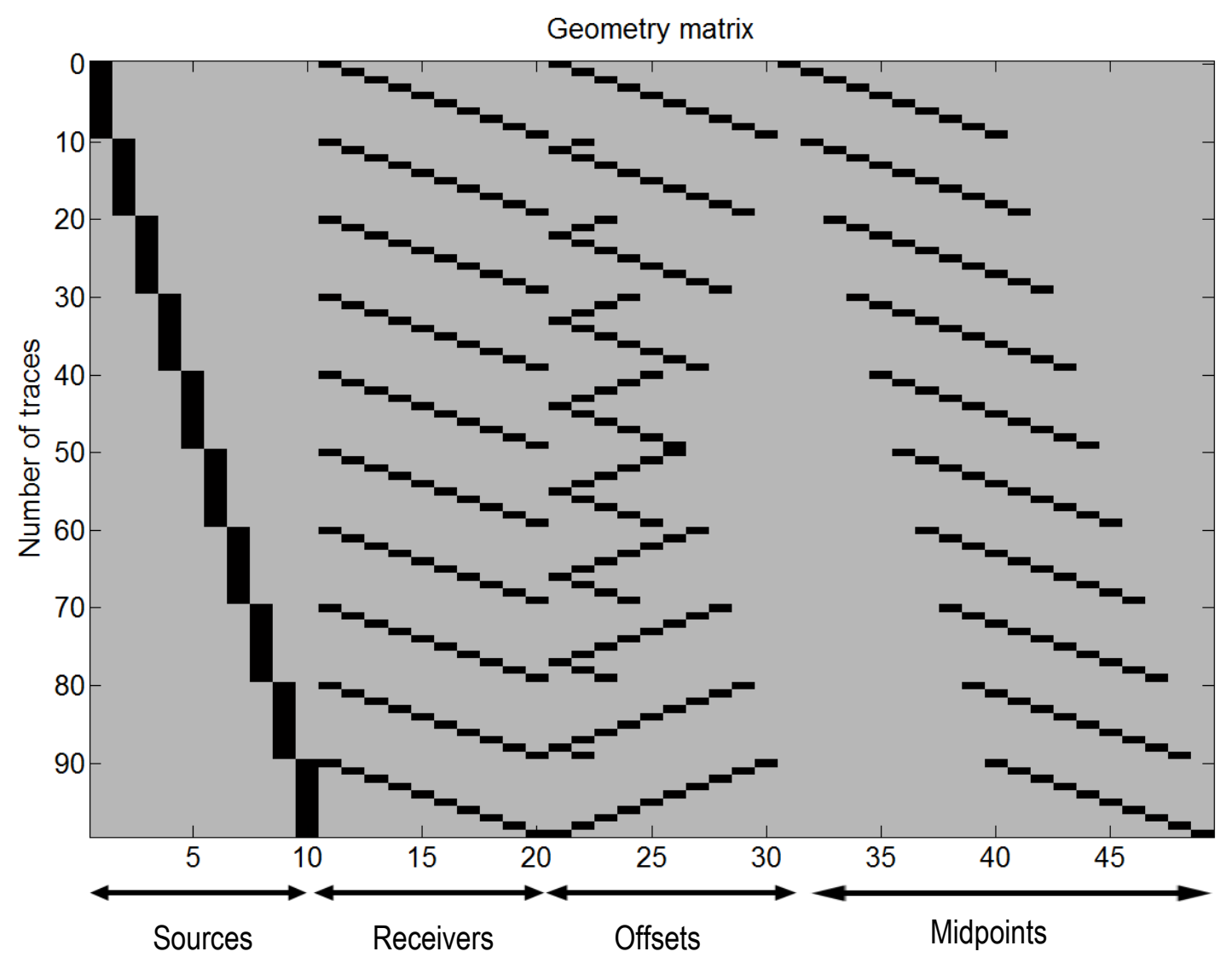

Figure 2.5: Geometry matrix of the synthetic data where the columns represent the sources, receivers, offsets, and midpoints. The rows represents the total number of traces. 
The least-squares solution to equation 2.3 must satisfy the following requirement

$$
E=\sum_{i, j}\left[T_{i j}-\left(S_{i}+R_{j}+Y_{l}+M_{l} X_{i j}^{2}\right)\right]^{2},
$$

where the error (E) is required to be minimum (Cox, 1999).

Since the statics problem has more equations than unknowns, the general inverse theory solves it by finding the best least-squares solution

$$
\mathbf{x}=\left(\mathbf{G}^{T} \mathbf{G}\right)^{-1} \mathbf{G}^{T} \mathbf{t}
$$

where $\mathbf{G}^{T}$ is the transpose of matrix $\mathbf{G}$.

In general, an $m \times n$ matrix $\mathbf{G}$, can be considered as a mapping from the model space, $S(m)$, to the data space, $S(D)$. There always exists a matrix decomposition called singular value decomposition (SVD) (Lancsoz, 1961) such that

$$
\mathbf{G}=\mathbf{U} \Lambda \mathbf{V}^{T}
$$

$\mathbf{U}(m \times m)$ is the matrix of eigenvectors of $\mathbf{G G}^{T}$ that span the data space, and $\mathbf{V}(n \times n)$ is the matrix of eigenvectors of $\mathbf{G}^{T} \mathbf{G}$ that span the model space. The singular values of the matrix $\mathbf{G}$ are the positive square roots of the eigenvalues of the matrix $\mathbf{G}^{T} \mathbf{G} . \Lambda$ is a diagonal matrix with the singular values of the matrix $\mathbf{G}$ in the diagonal elements in a decreasing order. Note that some of the singular values maybe zero. Then, the SVD of G simplifies into the compact form (Aster et al., 2005):

$$
\mathbf{G}=\mathbf{U}_{p} \Lambda_{p} \mathbf{V}_{p}^{T}
$$

where $\mathbf{U}_{p}$ and $\mathbf{V}_{p}$ denote the first $p$ columns of $\mathbf{U}$ and $\mathbf{V}$, related to non-zero singular values.

The problem of solution nonuniqueness, or underconstrained as highlighted by Wiggins et al. (1976) in seismic residual statics, is critical in the general inverse theory. Least-squares inverse theory provides an inverse solution that always exists and properly 
accommodates the rank and the dimension of $\mathbf{G}$, if $\mathbf{G}^{T} \mathbf{G}$ is invertible. Wiggins et al. (1976) and others have shown that the surface-consistent geometry matrix, G, has a nontrivial null space. This means that solutions (other than the null vector) to equation

$$
\mathrm{Gx}_{0}=\mathbf{0}
$$

exist (e.g., Aster et al. (2005)). Such a null solution can be added to any trial solution, $\mathbf{x}$, to the surface-consistent problem and we will obtain a model that fits the data equally well. In other words, if

$$
\|\mathbf{G x}-\mathbf{d}\|=\min
$$

then let's consider

$$
\left\|\mathbf{G}\left(\mathbf{x}+\mathbf{x}_{0}\right)-\mathbf{d}\right\|=\left\|\mathbf{G x}+\mathbf{G x}_{0}-\mathbf{d}\right\|=\|\mathbf{G x}-\mathbf{d}\|
$$

Wiggins et al. (1976) characterize the nontrivial null solutions as being long wavelength in nature. Long wavelengths are those waves that are longer than the cablelength (maximum offset).

Because $\mathbf{G}^{T} \mathbf{G}$ is singular due to the data being underconstrained, adding a small factor $\alpha$ stabilizes the inversion. This modification to equation 2.6 is known as damped least-squares solution. The new form is:

$$
\mathbf{x}=\left(\mathbf{G}^{T} \mathbf{G}+\alpha^{2} \mathbf{I}\right)^{-1} \mathbf{G}^{T} \mathbf{t}
$$

where $\mathbf{I}$ is the identity matrix.

In addition to the issue of small singular values, short and long wavelengths affects the statics solution. The statics solution is accurate for small wavelengths (less than a cablelength) where, in an iterative approach, the solution converges in very few iterations. However, statics correction of long wavelengths would be less accurate particularly in the presence of random noise. Even with many iterations, the solution may be exact 
mathematically but could have large uncertainties due to unavoidable random errors in picking (Wiggins et al., 1976).

The inversion given by equation 2.12 is called direct inversion which is often impractical to solve for very large number of equations, i.e $3 D$ data sets. Iterative methods, such as Gauss-Seidel or the conjugate gradient approach, are used to solve such large systems.

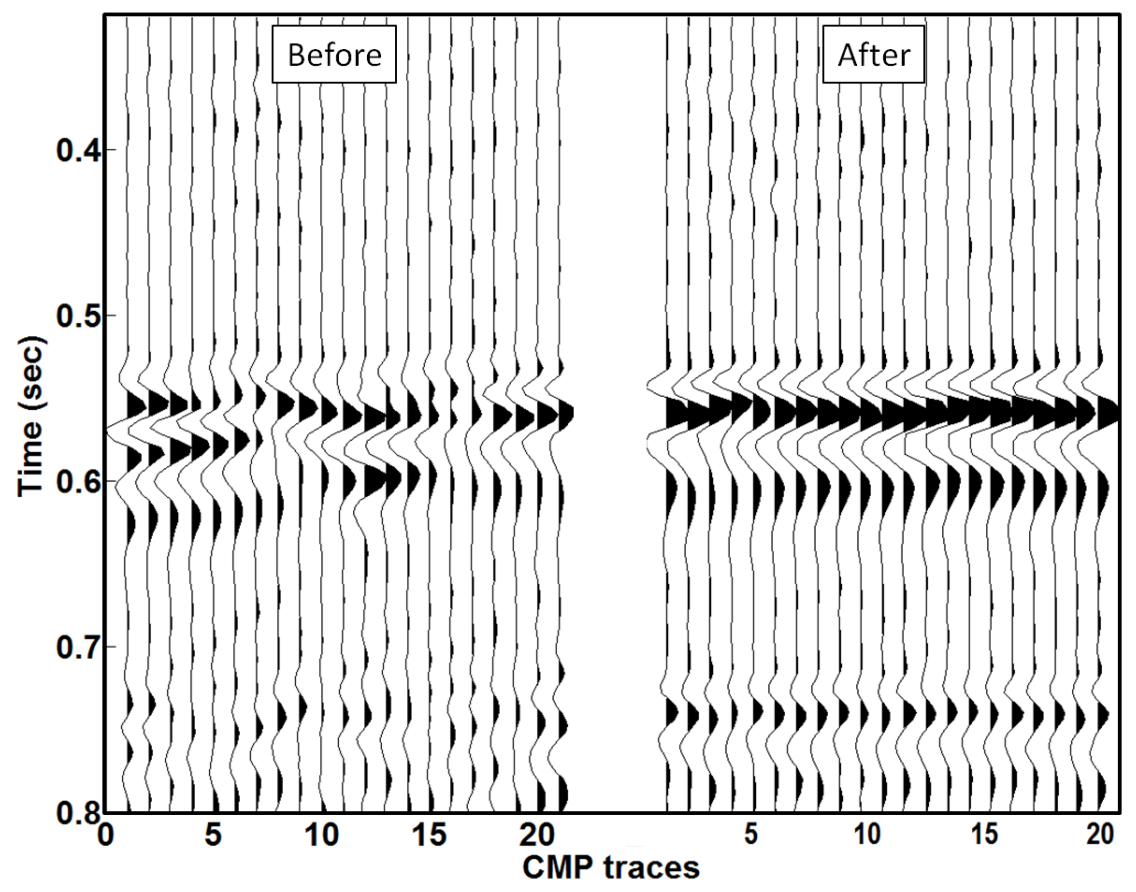

Figure 2.6: CMP stacks before and after surface-consistent residual statics.

In summary, the surface-consistent residual static corrections involves the following three steps:

1. computing the relative time shifts $T_{i j}$ by crosscorrelation, then

2. using the surface-consistent model to approximate $T_{i j}$ then decompose it into the four surface and subsurface consistent terms; and finally

3. applying the surface-consistent time shifts to the traces. 


\subsubsection{Deconvolution}

"Deconvolution is removing the effects of filtering from a filtered waveform" (Sheriff, 2002).

Deconvolution compresses the basic wavelet in the recorded trace thus improving the resolution and yielding bandlimited reflectivity that represents the subsurface (Yilmaz, 2001). Wavelet compression can be achieved using an inverse filter design using the leastsquares method. The inverse filter is a deconvolution operator designed to convert the seismic wavelet to a bandlimited spike through the convolutional process. It is based on the simple convolutional model of the recorded seismic trace, $d(t)$,

$$
d(t)=w(t) * r(t)+n(t)
$$

where $w(t)$ is a source wavelet, $r(t)$ represents the earth's impulse response, and $n(t)$ is the random ambient noise. This is a simple model that assumes the earth consists of a finite number of layers and that a traveling waveform reflects back at normal incidence.

The goal of deconvolution is to remove the effects the wavelet shape from the seismic trace. In other words, because the convolutional model replaces each reflectivity spike by a wavelet, and since the times between these reflectivity spikes are shorter than a wavelet length, constructive and destructive interference between wavelet shapes is bound to happen. Deconvolution attempts to compress these wavelets in time and hence resolve each reflection.

Single-channel deconvolution is based on the assumption that reflectivity series are white which usually is a sufficient assumption. However, it is sensitive to random noise due to the fact that it is an inverse operator.

Taner and Coburn (1980) and Taner and Koehler (1981) decreased this sensitivity to noise by computing the arithmetic mean of the amplitude spectrum for traces from a common shot gather whereby a single deconvolution operator is computed to remove 
the source effect. Thus surface-consistent deconvolution is a multichannel deconvolution which can be expanded to include statistical means of other different gathers, such as common receivers, common offsets, and common midpoints (Figure 2.7). A postulated surface-consistent deconvolution model form is:

$$
d_{i j}(t) \approx s_{i}(t) * r_{j}(t) * h_{k}(t) * y_{l}(t)
$$

where $d_{i j}$ is a model of the recorded seismic trace, $s_{i}$ is the waveform component associated with source location $i, r_{j}$ is the component associated with receiver location $j, h_{k}$ is the component associated with offset where index $k=|i-j|$, and $y_{l}$ represents the midpoint component $[l=(i+j) / 2]$.

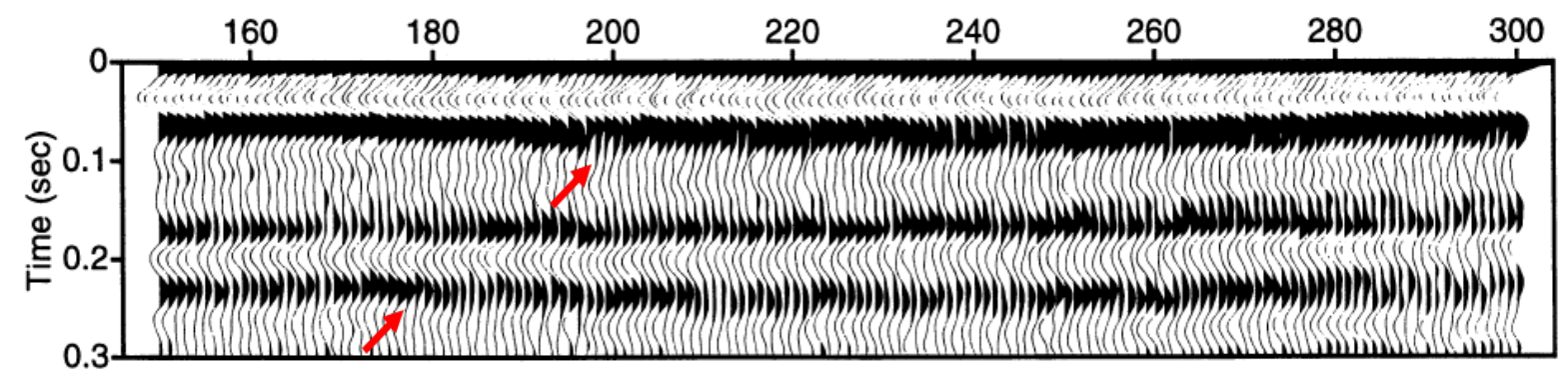

(a)

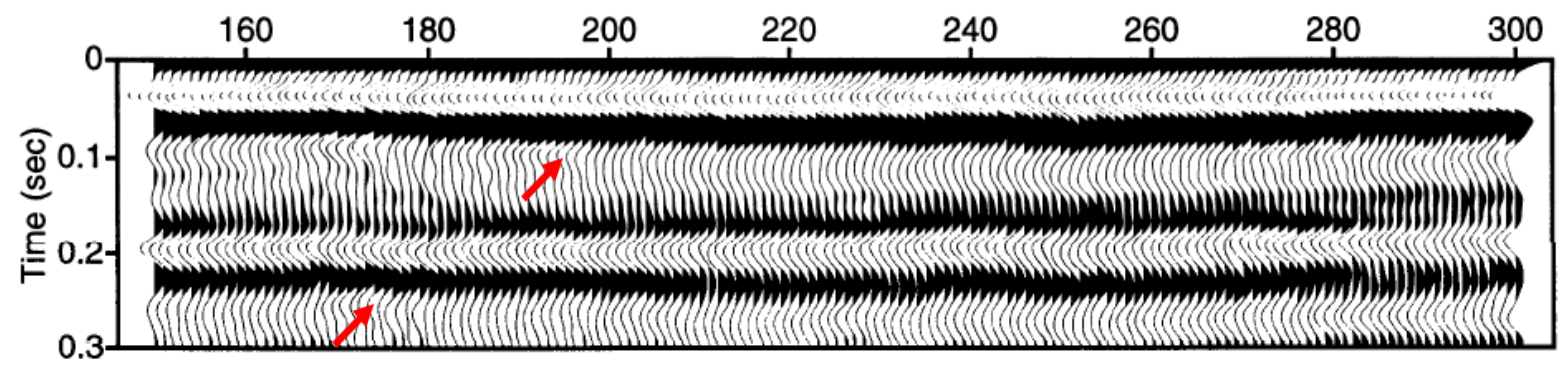

(b)

Figure 2.7: Stacked CMP sections showing the difference between single channel deconvolution in (a) compared to multichannel surface-consistent deconvolution in (b) (Hart, 1997).

For Levin (1989), working in the time-domain to design surface-consistent deconvolution filters made sense due to its stability and efficiency. On the other hand, many others chose to work in the log/Fourier domain stating that it is simpler and the prob- 
lem reduces to a linear system (Taner and Koehler, 1981; Morley and Claerbout, 1983; Cambois and Stoffa, 1992; Cary and Lorentz, 1993).

Fourier transforming equation 2.14, we get:

$$
D_{i j}(\omega) \approx S_{i}(\omega) R_{j}(\omega) H_{k}(\omega) Y_{l}(\omega),
$$

and taking the logarithm of equation 2.15, the problem becomes linear:

$$
\ln D_{i j} \approx \ln S_{i}+\ln R_{j}+\ln H_{k}+\ln Y_{l} .
$$

Equation 2.16 reduces the number of independent filter operators from $N_{t}$ to $\left(N_{s}+\right.$ $\left.N_{r}+N_{h}+N_{y}\right) . N_{s}$ is the total number of shots, $N_{r}$ is the total number of receivers, $N_{h}$ is the total number of offsets, $N_{y}$ is the total number of midpoints, and $N_{t}=N_{s} N_{c}$ represents the total number of traces $\left(N_{c}\right.$ is total number of channels). This is a very significant reduction considering the number of operators to solve for and the storage required for the computations. The least-squares solution for equation 2.16 is analogous to the residual statics correction problem discussed in the previous section. There are more equations, $N_{t}$, than unknowns, $N_{s}+N_{r}+N_{h}+N_{y}$. In linear algebra, this form of matrix systems is called overdetermined. This system is also known for being underconstrained in the long-wavelength components of the solution which means there is a deficiency in the number of independent equations available to solve for the unknowns.

The surface-consistent deconvolution problem in equation 2.16 can be solved using equation 2.4 , where $\mathbf{t}$ is replaced with $\mathbf{d}$ (a single vector, $N_{t}$, for every frequency in the data). The use of equation 2.12 has some advantages, one of which is that no iteration is required, and there is no subsequent problem with data storage. The problem of inverting large matrices can be significant, but for a small problem, this can be a quick and easy way of solving the least-squares system.

Other iterative methods, like the Gauss-Seidel, have been used to solve the deconvolution equation 2.16 (Morley and Claerbout, 1983; Cary and Lorentz, 1993). This type 
of decomposition is preferred for a large data set, and is the most widely used, where it iterates sums over shot, receiver, offset, and CMP gathers.

\subsubsection{Other applications}

The surface-consistent model discussed in equation 2.14 has been at the heart of several processing algorithms. In addition to the residual statics correction and deconvolution, amplitude balancing (Taner and Koehler, 1981), phase correction (Taner and Koehler, 1981; Cambois and Stoffa, 1993), and Gabor deconvolution (Montana and Margrave, 2006) all use the surface-consistent model. Yet another application of the surfaceconsistent model was presented by Nedlin (1985) to suppress multiples.

\subsection{Accuracy of the model}

The surface-consistent model is based on some assumptions (Section 2.2.2) made to make the computation easy and reasonably accurate. The accuracy of the model is difficult to quantify when processing field seismic data since the true earth model is unknown. The mathematical formulation of equation 2.1 is quite simple (reflecting the assumptions

made) and even if a data set is noise-free, the data will never be described exactly by this equation. Following is an example demonstrating this phenomenon.

A synthetic model (forward model) is presented in Figure 2.8 where the traces are formed by equation 2.14 and only the source term is allowed to vary. The other terms are all unit impulses. The geometry of this example consists of 10 shots with $10 \mathrm{~m}$ spacing, and 10 receivers per shot at $10 \mathrm{~m}$ spacing. The total number of traces is 100 . Figure 2.5 shows the geometry matrix, $\mathbf{G}$, for this example.

We reversed the process (inverse model) by modeling the 100 traces into their four surface-consistent terms as shown in Figure 2.9. The output model obtained from decomposing all traces into their four terms looks different from the model in Figure 2.8. 
However, when we convolved the four output terms shown in Figure 2.9 we get a new set of traces which we compared with the traces obtained from the forward modeling. Figure 2.10 shows the forward model traces, inverse model traces, and their difference. We can compute this difference (RMS of the difference) and express it in percentage:

$$
\operatorname{RMSD}(\%)=100 * \sqrt{\frac{\sum_{t}\left(d_{2}-d_{1}\right)^{2}}{\sum_{t}\left(d_{1}\right)^{2}}},
$$

where $d_{1}$ and $d_{2}$ are two signals. Figure 2.11 shows the error between the seismic data of the forward modeling and the seismic generated from the inverse modeling. In general, the error is very low except for those traces generated from shot number 2 .

In addition to the data difference, we examine the model variation (the four surfaceconsistent terms) by taking the ratio of the sum of power of two adjacent filters, as shown in Figure 2.12. By taking the ratio of two adjacent filters, we are canceling out the longwavelength effects. The difference between the input model and the output model is minimum (except for shot number 2), however it highlights an important observation, the leakage to other components, where the three terms that are supposed to be unit impulses are not exactly so in the inverse model.

The accuracy of the model depends strongly on the signal-to-noise ratio of the data. The higher that ratio, the more reliable the approximation of the surface-consistent model.

\subsection{Summary}

We presented a review of the surface-consistent model, its basic assumptions, its basic equation, and a review of the two applications of the surface-consistent model practiced in exploration geophysics. The surface-consistent model conceptually separates the nearsurface effects from the subsurface effects through the dependence of the data on the

acquisition coordinates. The model represents the trace as a convolutional model of 


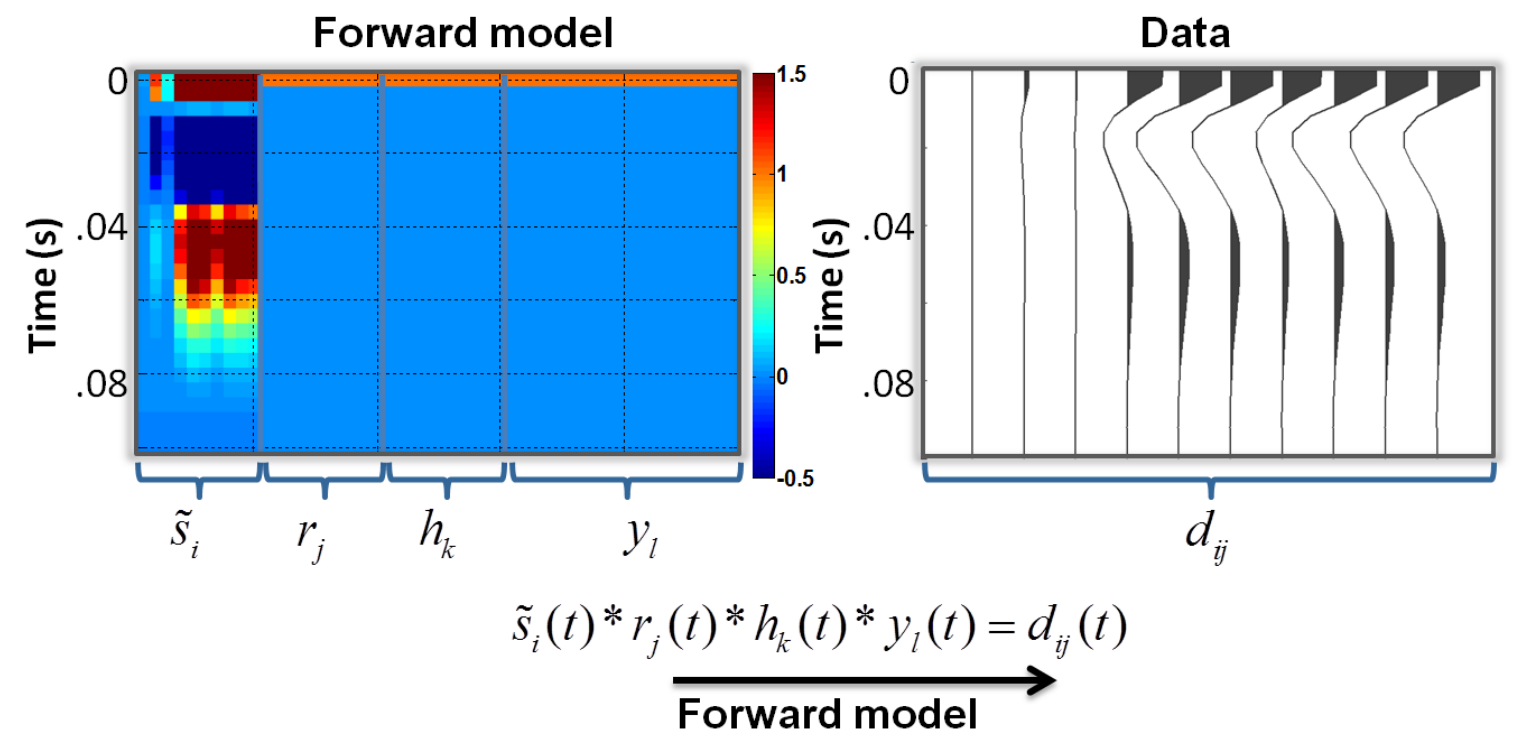

Figure 2.8: Forward modeling of the surface-consistent model with variations in the source term only.

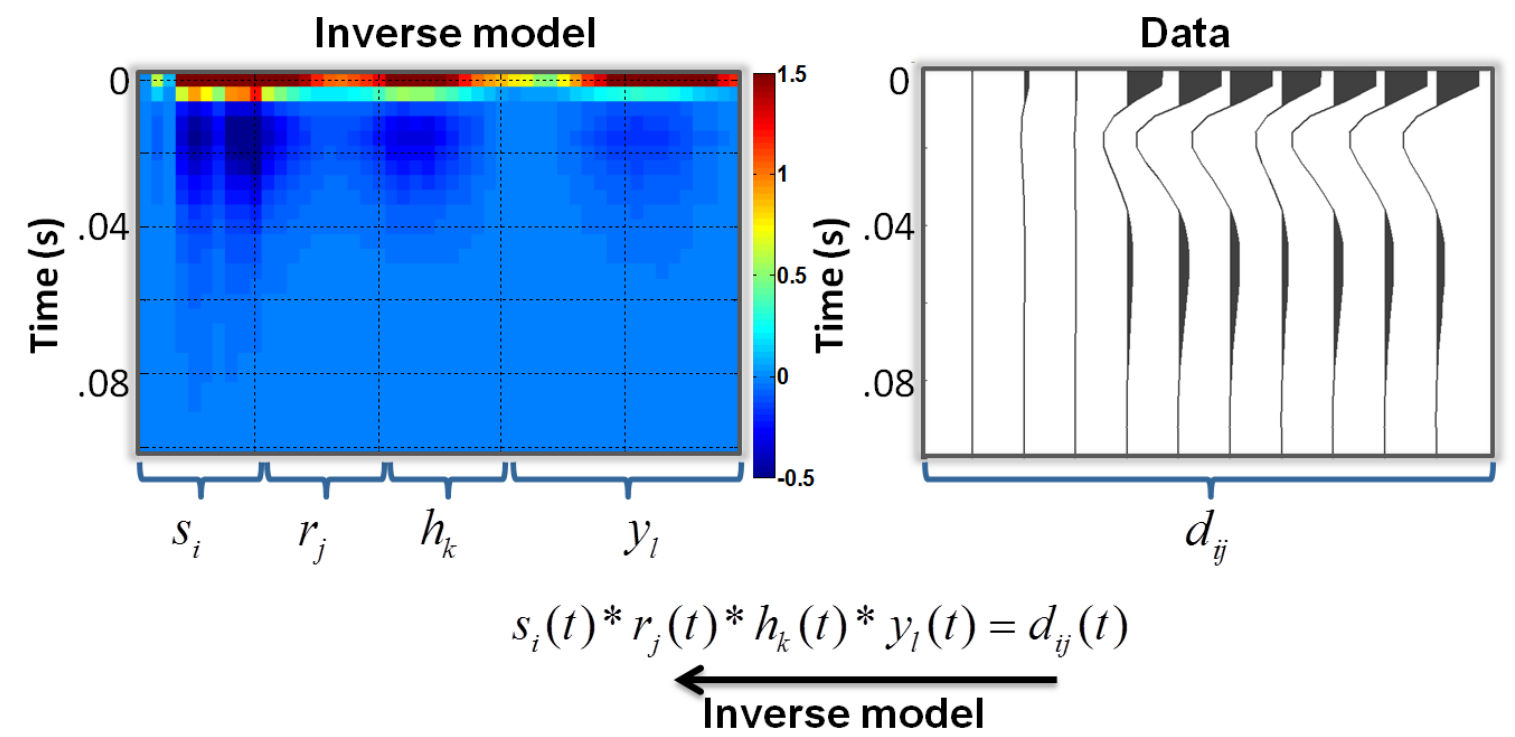

Figure 2.9: Inverse modeling of the surface-consistent model. Deconvolving the same seismic traces in Figure 2.8 results in a different model than the input model of the forward operation. There is a leakage of the solution to other components. 


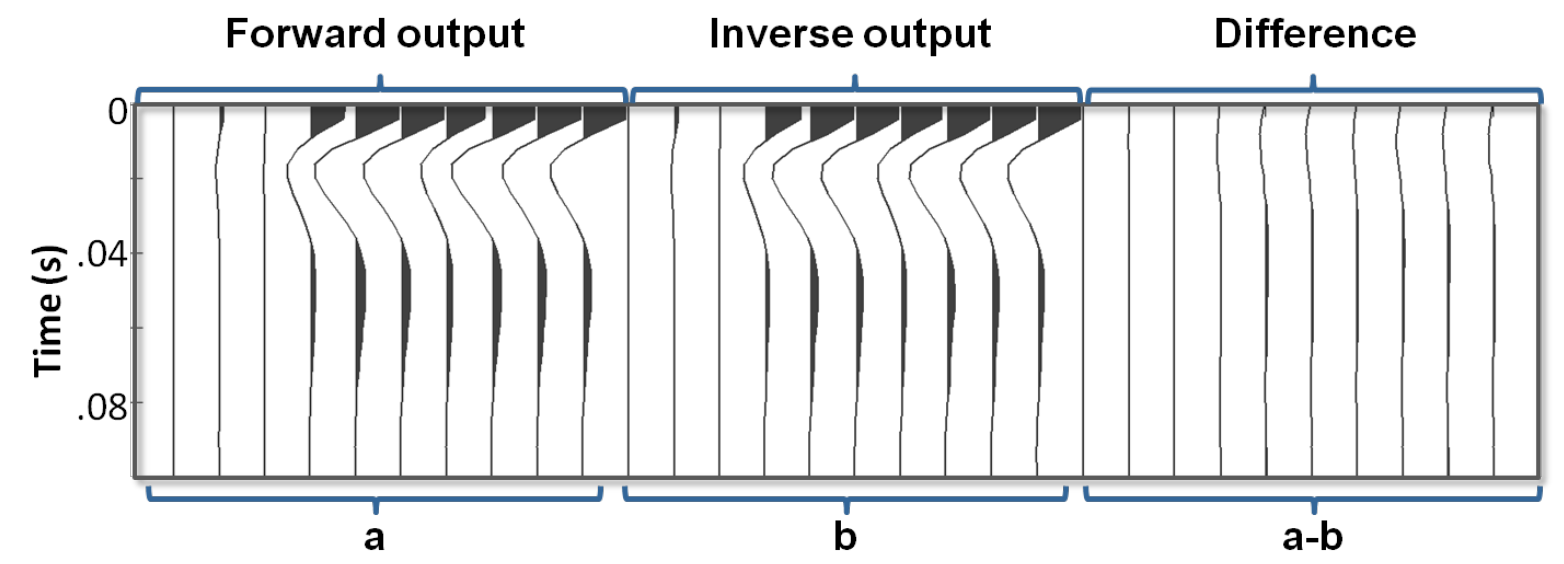

Figure 2.10: Seismic data of the forward model (a), and seismic data of the inverse model (b), and their difference. Note the difference is minimum even though the two models are different.

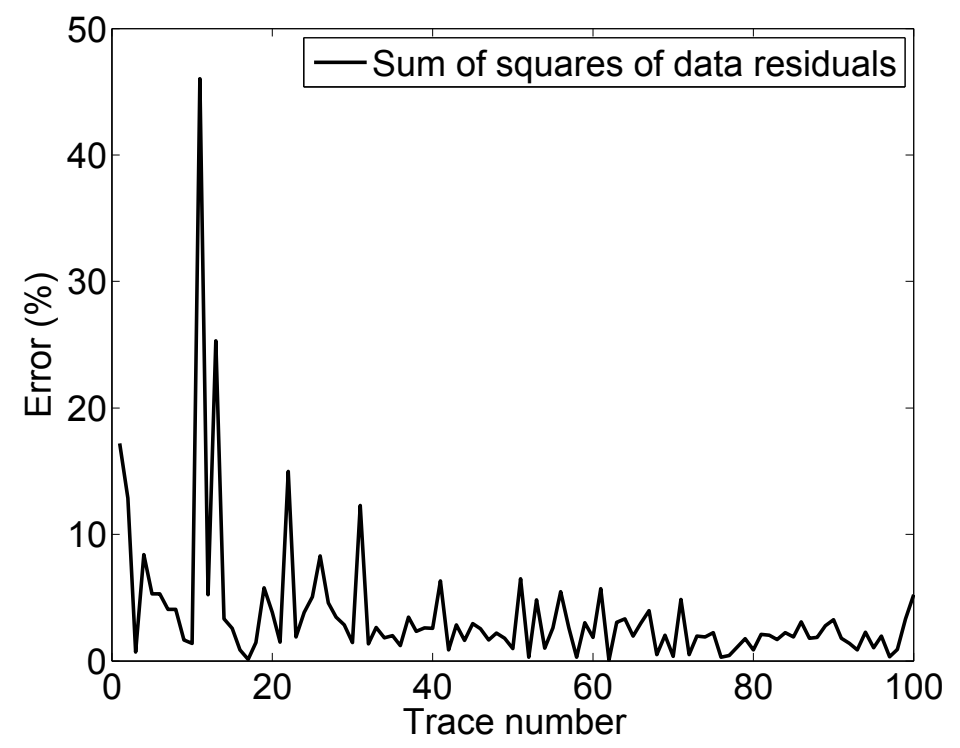

Figure 2.11: The sum squares of the residual of the traces generated from the input model (Figure 2.8) and the output traces generated from the output model (Figure 2.9). 


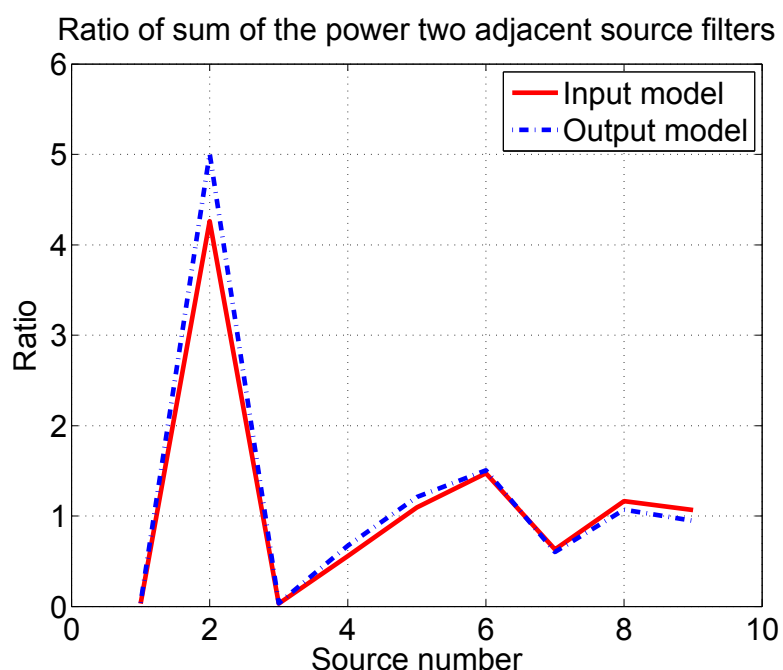

(a)

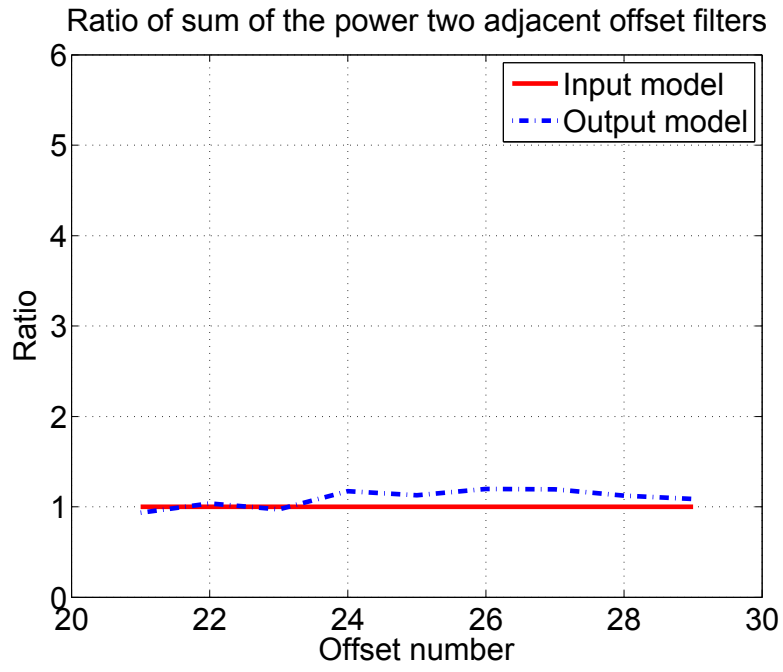

(c)

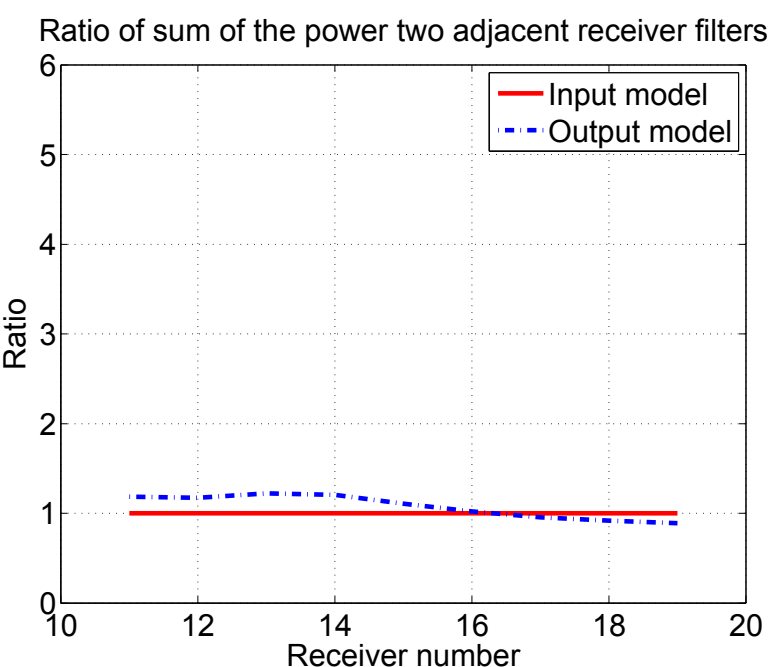

(b)

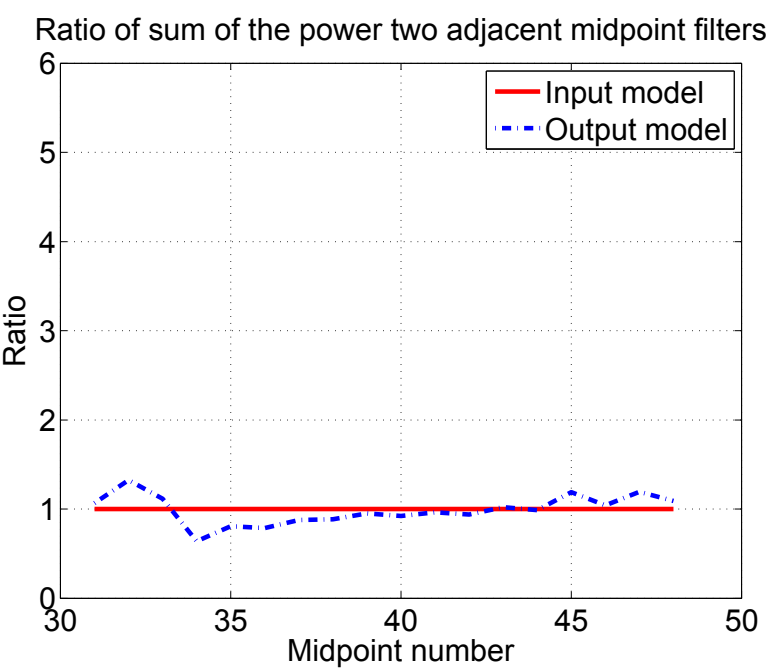

(d)

Figure 2.12: Comparisons of the two adjacent filters of the input model (Figure 2.8) and the output model (Figure 2.9). (a) is the source ratio, (b) is the receiver ratio, (c) is the offset ratio, and (d) is the midpoint ratio. 
its four components, two surface components and two subsurface components. The two surface terms approximate the surface and the near-surface factors affecting traces related to a specific source and a specific receiver. Similarly, the offset and midpoint terms approximate the subsurface effects. Although the surface-consistent model is widely used in seismic data processing, it is, nevertheless, an approximate trace model.

We also presented a review of two applications of the surface-consistent model in exploration geophysics, that is the statics problem and deconvolution. Both applications are important in seismic data processing and their use is very common. Other applications of the model are mentioned. The accuracy of the surface-consistent model is illustrated with a simple noise-free example. 


\section{Chapter 3}

\section{Extending the surface-consistent hypothesis to model two seismic surveys}

\subsection{Introduction}

In Chapter 2, we presented a review of the surface-consistent model, discussed its basic assumptions, and presented its basic mathematical form. Because of complexities of the earth's subsurface, few assumptions are made so that we can obtain a model of the subsurface that is easy to compute and reasonably accurate. This conceptual model separates near-surface effects from subsurface effects through the dependence of the data upon the acquisition coordinates. The four seismic acquisition coordinates are: common source, common receiver, common offset, and common midpoint. The model was introduced by Schneider (1971) as the basic approach for solving the statics problem. Taner et al. (1974) formalized the model based on the four trace planes, then Taner and Koehler (1981) presented the basic assumptions.

Although this model is not exact, it has proven to be successful in many applications of seismic data processing, such as: static corrections Taner et al. (1974) and Wiggins et al. (1976), deconvolution (Morley and Claerbout, 1983; Levin, 1989; Cambois and Stoffa, 1992; Cary and Lorentz, 1993), amplitude balancing (Yu, 1985; Taner and Koehler, 1981), phase rotation (Taner et al., 1991; Cambois and Stoffa, 1993), and multiple attenuation (Nedlin, 1985). These applications are available in commercial processing software and are commonly used in seismic data processing workflows.

In this chapter, we introduce a new concept that is based on, and is an extension of, the surface-consistent model. So far, the model has approximated a single data set. 
However, the concept we introduce here is based on two data sets instead on only one. This new extension is called "Surface-consistent matching filters" and is abbreviated SCMF.

Since a review of the surface-consistent model has been discussed in the previous chapter, we will focus in this chapter on the following:

1. The form of the extension in time and frequency domains,

2. the links between the new algorithm and the well known least-squares matching filter, and

3. the SCMF decomposition.

\subsection{Extending the surface-consistent hypothesis to model two seismic surveys}

Taner et al. (1974) and Taner and Koehler (1981) represented the trace as the convolution of four terms: the source response, the receiver response, the offset response, and the midpoint response (equation 2.1). We can rewrite the same equation with a subscript 1 to represent modeling traces from the first data set as follows:

$$
d_{1}(t) \approx s_{1}(t) * r_{1}(t) * h_{1}(t) * y_{1}(t)
$$

where we have suppressed the subscripts $i, j, k$, and $l$ that denote explicit surface locations and simply use the subscript 1 to refer to the first survey. Similarly, the corresponding trace from another seismic survey (with subscript 2) may be modeled as

$$
d_{2}(t) \approx s_{2}(t) * r_{2}(t) * h_{2}(t) * y_{2}(t)
$$

At the moment, let us assume that the two surveys have exactly the same geometry, that is the sources and the receivers are co-located and similarly the offsets and midpoints. 


\subsubsection{Frequency-domain form of the extended model}

Fourier transforming equations 3.1 and 3.2 , forming their ratio, and linearizing by taking the logarithm of both sides, we obtain

$$
\log \left(\frac{\widehat{d}_{1}(\omega)}{\widehat{d}_{2}(\omega)}\right) \approx \log \left(\frac{\widehat{s}_{1}(\omega)}{\widehat{s}_{2}(\omega)}\right)+\log \left(\frac{\widehat{r}_{1}(\omega)}{\widehat{r}_{2}(\omega)}\right)+\log \left(\frac{\widehat{h}_{1}(\omega)}{\widehat{h}_{2}(\omega)}\right)+\log \left(\frac{\widehat{y}_{1}(\omega)}{\widehat{y}_{2}(\omega)}\right),
$$

where $\omega$ is frequency, the "^" denotes the Fourier transform. The left-hand side of equation 3.3 is the data log spectral ratio and the right-hand side contains the sum of surface-consistent terms. Using equation 3.3 to form a linear system of equations, we can create a separate linear system for each frequency, where each such system has one equation per trace. Provided that the number of traces exceeds the number of shots plus the number of receivers plus the number of offsets plus the number of midpoints, equation 3.3 can be used to build an overdetermined system of linear equations whose least-squares solution estimates the desired surface-consistent terms. In the next section, we will show that the Fourier transform of a matching filter is a spectral ratio. Hence, the spectral ratios in equation 3.3 define a set of four-component surface-consistent matching filters.

\subsubsection{Analogy to matching filters}

Another well-known concept utilized in this study is the shaping or matching filter (Claerbout, 1976, p.130-133; Robinson and Treitel, 1980, ch.1, 8, and 14). This is a filter designed to alter the shape of an input signal in order to obtain a desired output signal. In other words, it is a convolutional filter that minimizes the sum-squared difference between two signals.

Sheriff (2002) defined matching filter as one "which maximizes the output in response to a signal of particular shape." In geophysical literature, terms such as the Wiener least-square filtering, matching or shaping filters (Robinson and Treitel, 1980; Claerbout, 
1976), crosscorrelation filters (Anstey, 1964), and correlator (Karl, 1989), to name just a few, all share the same definition.

Following Robinson and Treitel (1980) notations, we consider the problem of finding a filter $f_{t}=\left(f_{0}, f_{1}, \ldots, f_{n}\right)$ of length $n+1$ that shapes an input waveform $b_{t}=\left(b_{0}, b_{1}, \ldots, b_{m}\right)$ of length $m+1$ into a desired output trace $d_{t}=\left(d_{0}, d_{1}, \ldots, d_{m+n}\right)$ of length $m+n+1$ so that the error between the desired output $d_{t}$ and actual output $c_{t}$ is minimum. The actual output is written as follow:

$$
c_{t}=\sum_{s=0}^{n} f_{s} b_{t-s},
$$

where this is known as the convolution of the shaping filter with the input signal.

We can formulate the design equation for the match filter as a general inverse problem such that

$$
\mathrm{Bf}=\mathrm{d}
$$

where $\mathbf{B}$ is the convolution matrix formed from trace $\mathbf{b}, \mathbf{f}$ is the computed matching filter, and $\mathbf{d}$ is the least-squares desired output trace. Our notation convention for matrices are upper case boldface and vectors are lower case boldface. The expression for the residual vector, $\mathbf{e}$, is

$$
\begin{aligned}
\mathbf{e} & =\mathrm{Bf}-\mathbf{d} \\
& =\mathbf{c}-\mathbf{d},
\end{aligned}
$$

where $\mathbf{c}$ is the actual trace. The convolution matrix (or Toeplitz matrix), $\mathbf{B}$, can be 
expressed in matrix form

$$
\left[\begin{array}{ccccccc}
b_{0} & 0 & 0 & \cdot & \cdot & \cdot & \cdots \\
b_{1} & b_{0} & 0 & 0 & \cdot & \cdot & \cdots \\
b_{2} & b_{1} & b_{0} & 0 & 0 & \cdot & \cdots \\
\cdot & \cdot & \cdot & b_{0} & 0 & \cdot & \vdots \\
\cdot & \cdot & \cdot & \cdot & \ddots & \cdot & \vdots \\
b_{m} & b_{m-1} & b_{m-2} & \cdot & \cdot & \cdots & b_{0}
\end{array}\right] .
$$

The number of columns of the convolution matrix depends on the length of the match filter, $\mathbf{b}$. The number of rows of $\mathbf{B}$ is the number of equations (equal to total trace length) which is more than the number of unknowns (equal to match filter length). The least-squares method is appropriate in such situation since the objective is not to design a perfect filter that matches both signals exactly.

Minimizing e in equation 3.6 in the least-squares sense results in solving the system of normal equations:

$$
\begin{aligned}
\mathbf{B}^{T} \mathbf{B f} & =\mathbf{B}^{T} \mathbf{d} \\
\mathbf{f} & =\left(\mathbf{B}^{T} \mathbf{B}\right)^{-1} \mathbf{B}^{T} \mathbf{d} .
\end{aligned}
$$

In equation 3.8, the filter coefficients of $\mathbf{f}$ are given by the cross-correlation of $\mathbf{b}$ with $\mathbf{d}$, filtered by the inverse of the autocorrelation of $\mathbf{b}$. Equation 3.8 can be written in matrix form

$$
\left[\begin{array}{cccccc}
\phi_{0} & \phi_{1} & \phi_{2} & \phi_{3} & \cdot & \phi_{n} \\
\phi_{1} & \phi_{0} & \phi_{1} & \phi_{2} & . & \cdot \\
\phi_{2} & \phi_{1} & \phi_{0} & \phi_{1} & \phi_{2} & \cdot \\
\phi_{3} & \phi_{2} & \phi_{1} & \phi_{0} & \phi_{1} & \vdots \\
\cdot & \cdot & . & \cdot & \ddots & \cdot \\
\phi_{m} & \phi_{m-1} & . & . & \cdots & \cdot
\end{array}\right]\left[\begin{array}{c}
f_{0} \\
f_{1} \\
\vdots \\
f_{n}
\end{array}\right]=\left[\begin{array}{c}
S_{0} \\
S_{1} \\
\cdot \\
\cdot \\
\vdots \\
S_{m}
\end{array}\right]
$$


where $\phi_{m}$ is the $m^{\text {th }}$ lag of the autocorrelation of $\mathbf{b}$ and $\mathbf{s}$ is the cross-correlation of $\mathbf{b}$ with $\mathbf{d}$.

Figure 3.1 shows two noise-free traces generated from the same reflectivity series but convolved with two different wavelets. There is a time delay ( $25 \mathrm{~ms})$, amplitude difference between both traces, and the computed NRMS error is about 153\% (details of NRMS is in Section 4.2). To design a match filter that matches Trace 2 to Trace 1, two parameters are required for an ideal filter: 1) filter length, and 2) an optimum lag. Different window types have been examined (i.e boxcar, gaussian, and hanning) for the time-domain match filter but the results show very little difference. Figure 3.2 illustrates Trace 1 and Trace 2 after computing and applying a time-domain match filter for a filter length equals to $0.4 \mathrm{~s}$ and using a gaussian window. This filter is expressed as:

$$
f(t) * d_{2}(t)=d_{1}(t),
$$

and the least squares solution is obtained by minimizing the error function defined by

$$
e=\sum_{t}\left(f(t) * d_{2}(t)-d_{1}(t)\right)^{2} .
$$

$f(t)$ is the time-domain matching filter which minimizes differences between two signals, $d_{1}$ and $d_{2}$.

The optimum lag between the two traces is determined using crosscorrelation technique, a time-shift (determined from the time lag at which the crosscorrelation function has a maximum value) is used to align both traces, then a match filter is computed. The NRMS error is 19\%, indicating a good match. This error depends on the filter length, where shorter filter lengths increase the error but with less computational cost.

A frequency domain match filter can also be obtained from matching $d_{2}$ to $d_{1}$. The form of this match filter can be determined from the spectral ratio of both traces. In the 
Trace 1
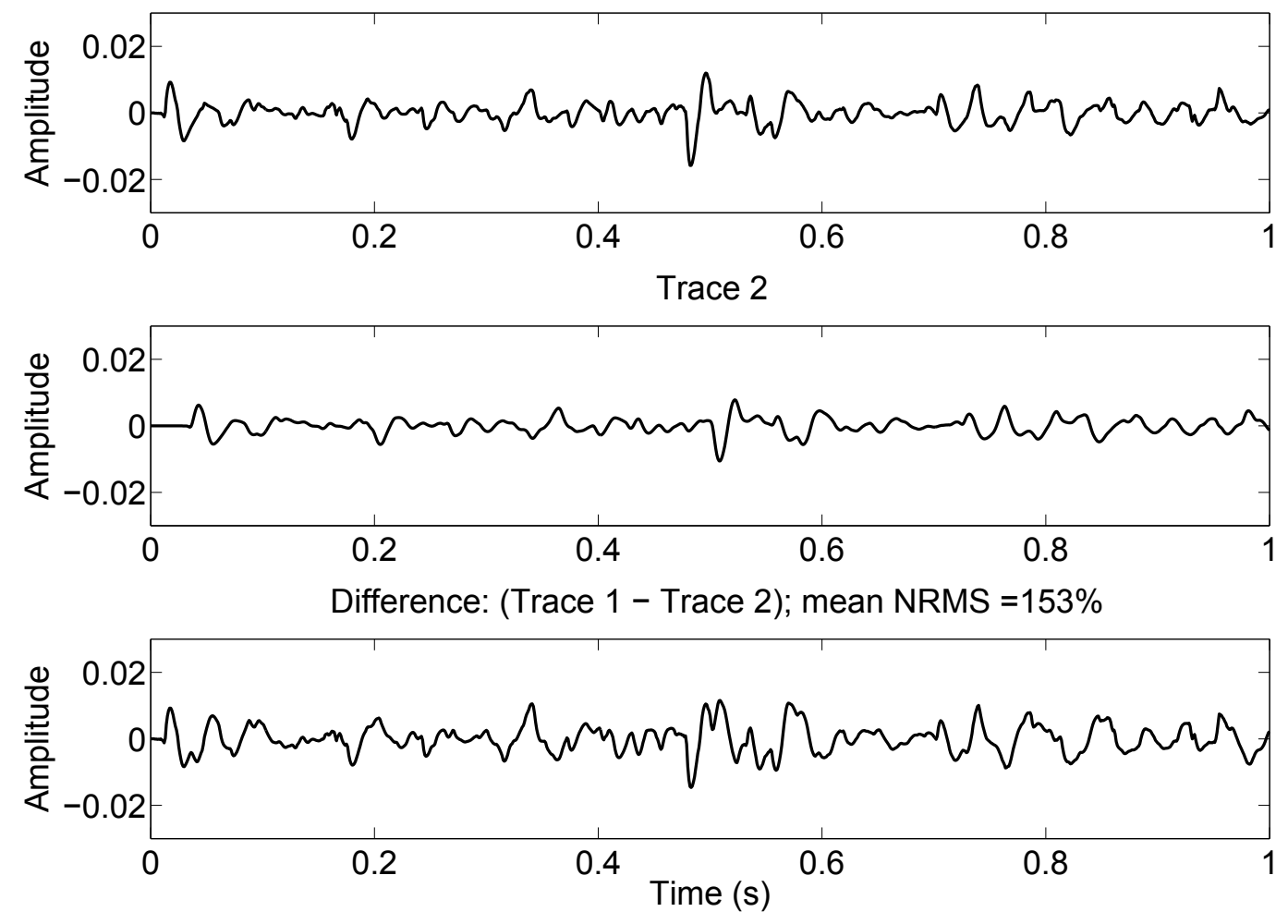

Figure 3.1: An example of two noise-free traces generated from the same reflectivity sequence but convolved with two different wavelets. The residual reflects the time-shift and the amplitude difference. 


\section{Trace 1}
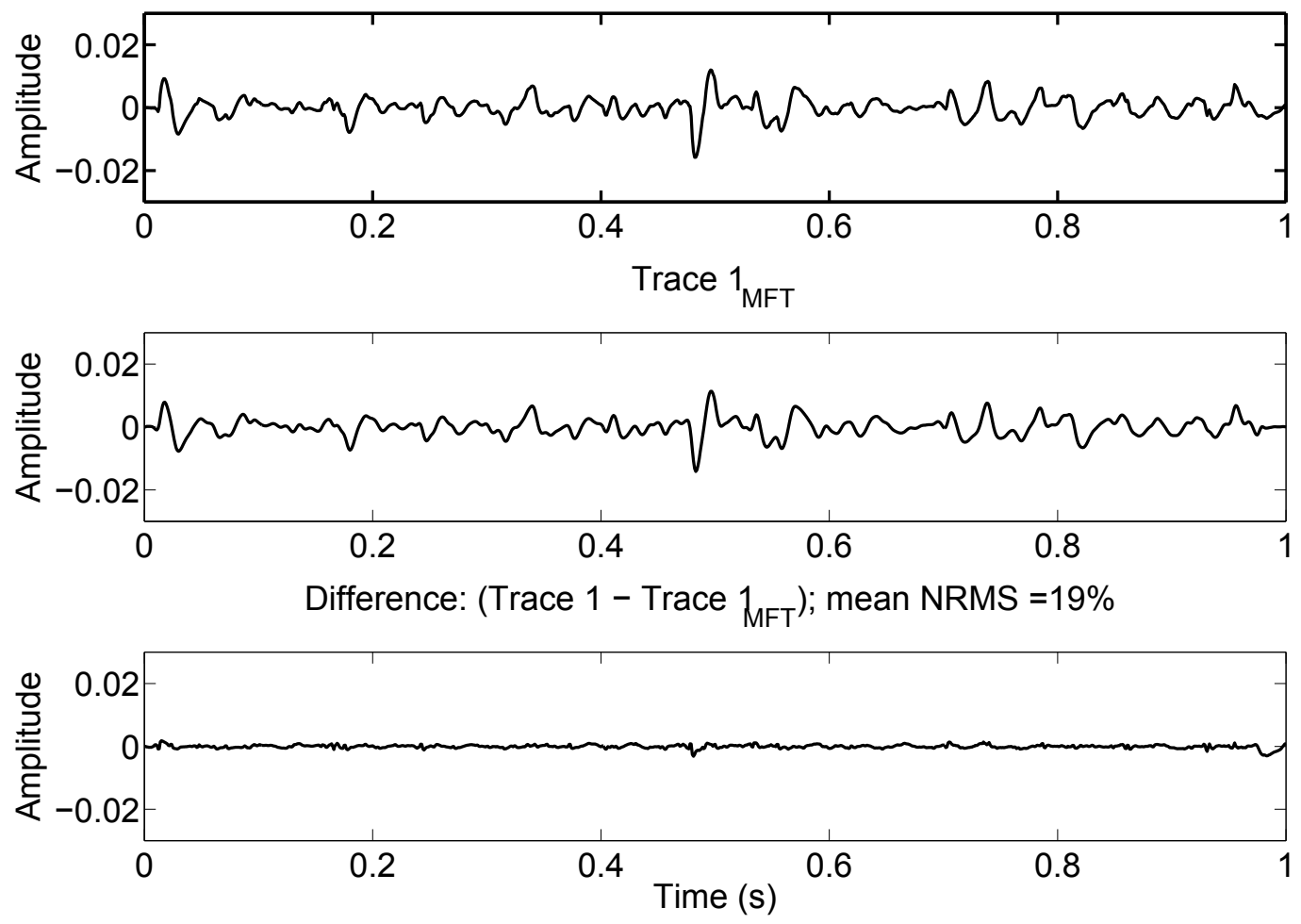

Figure 3.2: The same example shown in Figure 3.1 but after time-domain matching of Trace 2 to Trace 1 where the result is Trace $1_{M F T}$. 
frequency domain, equation 3.10 can be solved exactly as:

$$
\widehat{f}(\omega)=\frac{\widehat{d}_{1}(\omega)}{\widehat{d}_{2}(\omega)} .
$$

Figure 3.3 is the result of matching $d_{2}$ to $d_{1}$ using equation 3.12 . Compared to the timedomain match filter, the spectral ratio method is sensitive to the type of window used (particularly when noise exists), and an ideal one is a gaussian window (no zero crossing in the Fourier domain). Figure 3.4 illustrates these windows where the nominal width of each window is the filter length. Table 3.1 shows the match filter accuracy for the two noise-free traces for five filter lengths, and comparing the results of the match filter using the time-domain least-squares and the spectral ratio. The time-domain match filter shows lower NRMS error versus that of the spectral ratio method.

Table 3.1: Match filter accuracy for the two noise-free traces in Figure 3.1 comparing the time-domain least-squares and the spectral ratio for five filter lengths.

\begin{tabular}{|c|c|c|}
\hline \multirow{2}{*}{ Filter length (s) } & \multicolumn{2}{|c|}{ NRMS error (\%) } \\
\cline { 2 - 3 } & Time-domain LS & Spectral ratio \\
\hline 0.2 & 28 & 20 \\
0.4 & 19 & 26 \\
0.6 & 17 & 27 \\
0.8 & 16 & 26 \\
1.0 & 16 & 23 \\
\hline
\end{tabular}

Figure 3.5 shows the same traces in Figure 3.1 except a white gaussian noise has been added to both traces (signal-to-noise ratio $=2$ ). Figures 3.6 and 3.7 show the timedomain and spectral ratio match filter for the noisy traces. The results of both filters are similar, however, the spectral ratio computation required knowledge of both traces bandwidth which are different as illustrated in Figure 3.8, where the two horizontal lines define the approximate noise level for each spectrum. Table 3.2 shows a comparison of the error values of both match filter methods for the noisy traces for five filter lengths. The results are close for both methods. 
Trace 1
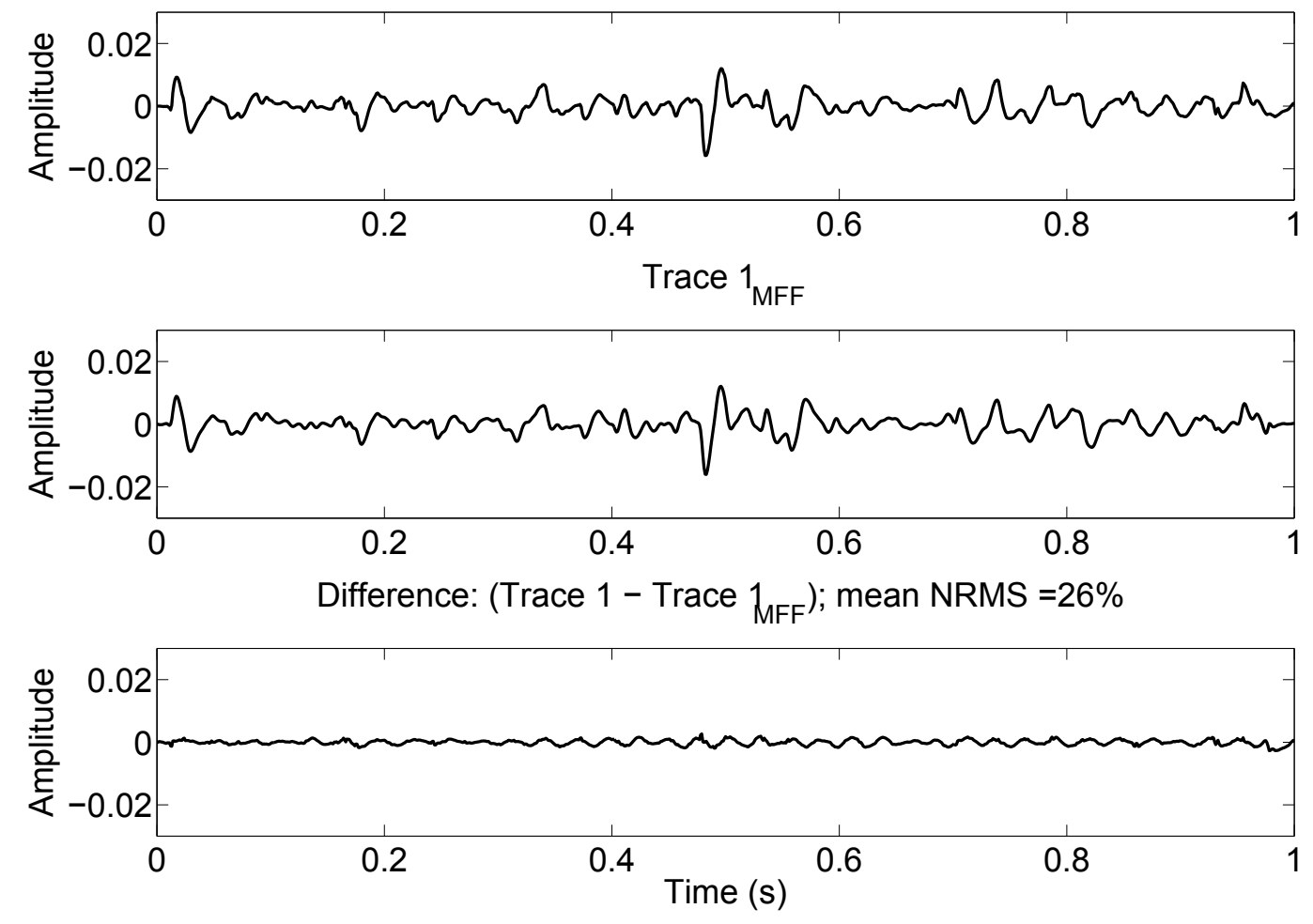

Figure 3.3: The same example shown in Figure 3.1 but after spectral ratio match filtering of Trace 2 to Trace 1 where the result is Trace $1_{M F F}$.

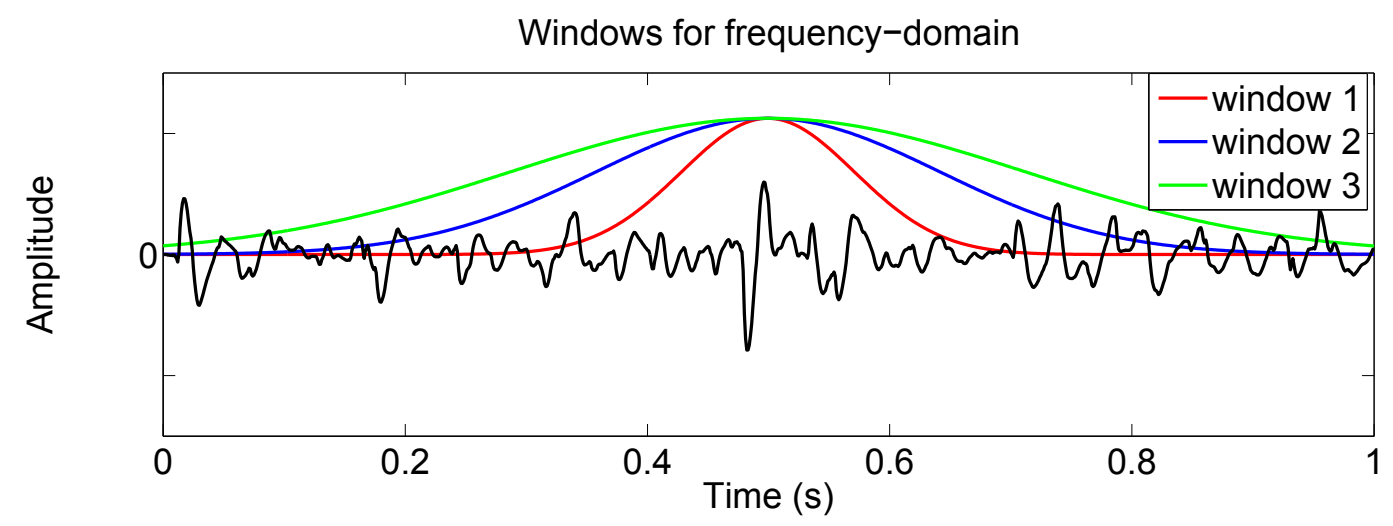

Figure 3.4: An example of three filter lengths used for the frequency-domain match filter. 
Trace 1
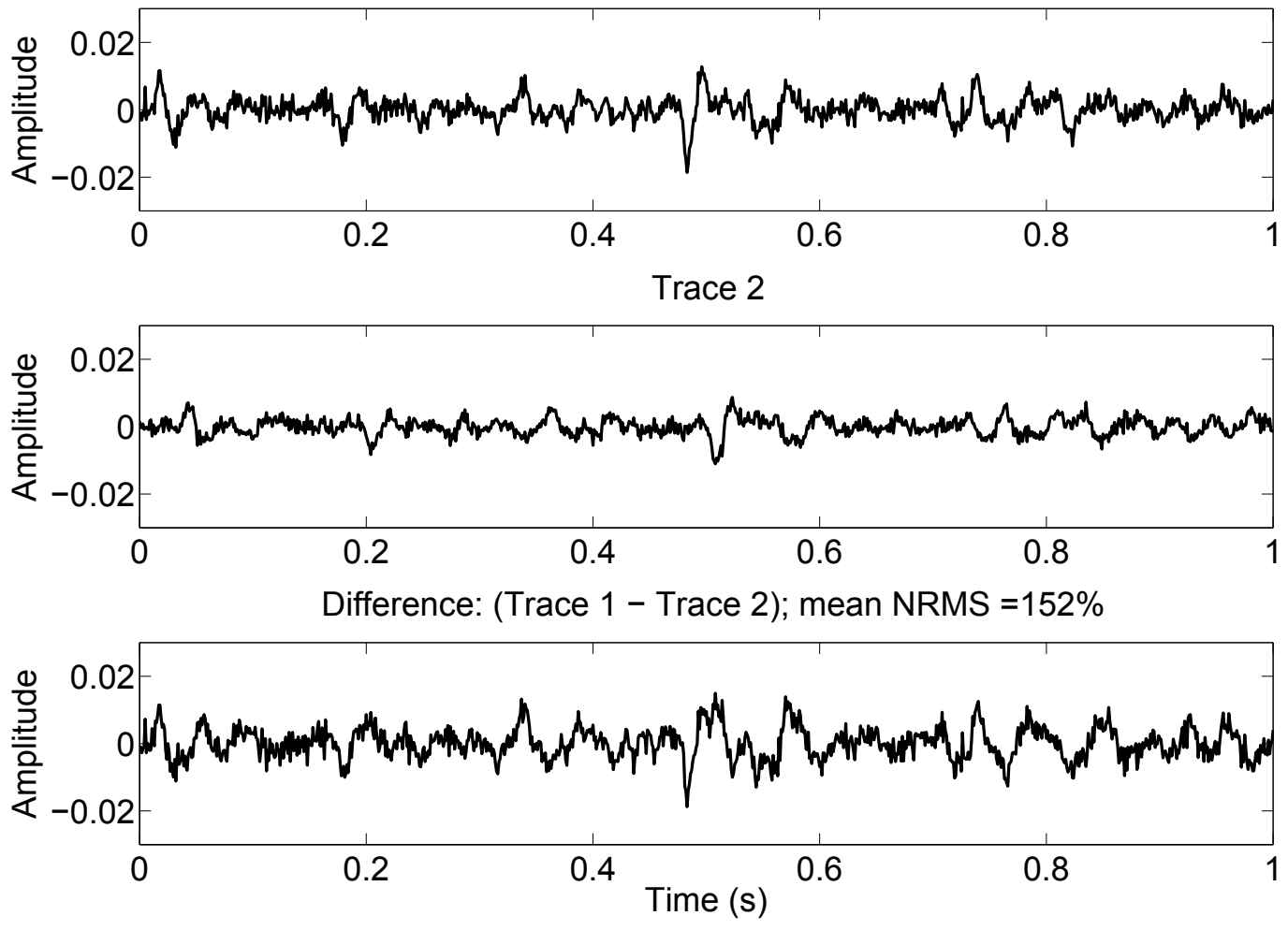

Figure 3.5: The same example shown in Figure 3.1 with added noise.

Table 3.2: Match filter accuracy for two noisy traces shown in Figure 3.5 comparing the time-domain least-squares and the spectral ratio for five filter lengths.

\begin{tabular}{|c|c|c|}
\hline \multirow{2}{*}{ Filter length (s) } & \multicolumn{2}{|c|}{ NRMS error (\%) } \\
\cline { 2 - 3 } & Time-domain LS & Spectral ratio \\
\hline 0.2 & 65 & 59 \\
0.4 & 57 & 59 \\
0.6 & 53 & 56 \\
0.8 & 50 & 51 \\
1.0 & 46 & 44 \\
\hline
\end{tabular}




\section{Trace 1}
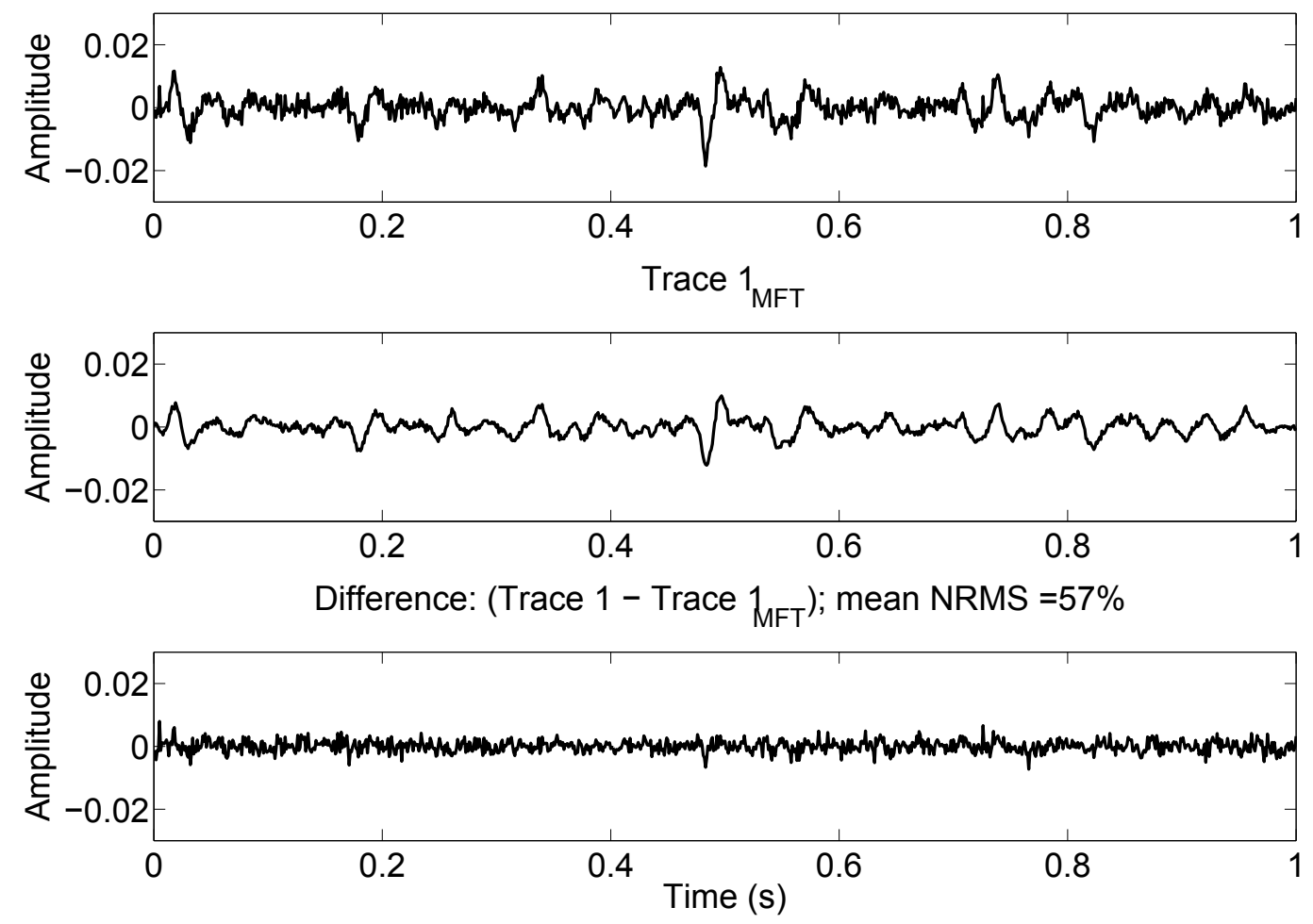

Figure 3.6: The time-domain matching of Trace 2 to Trace 1 where the result is Trace $1_{M F T}$. 
Trace 1
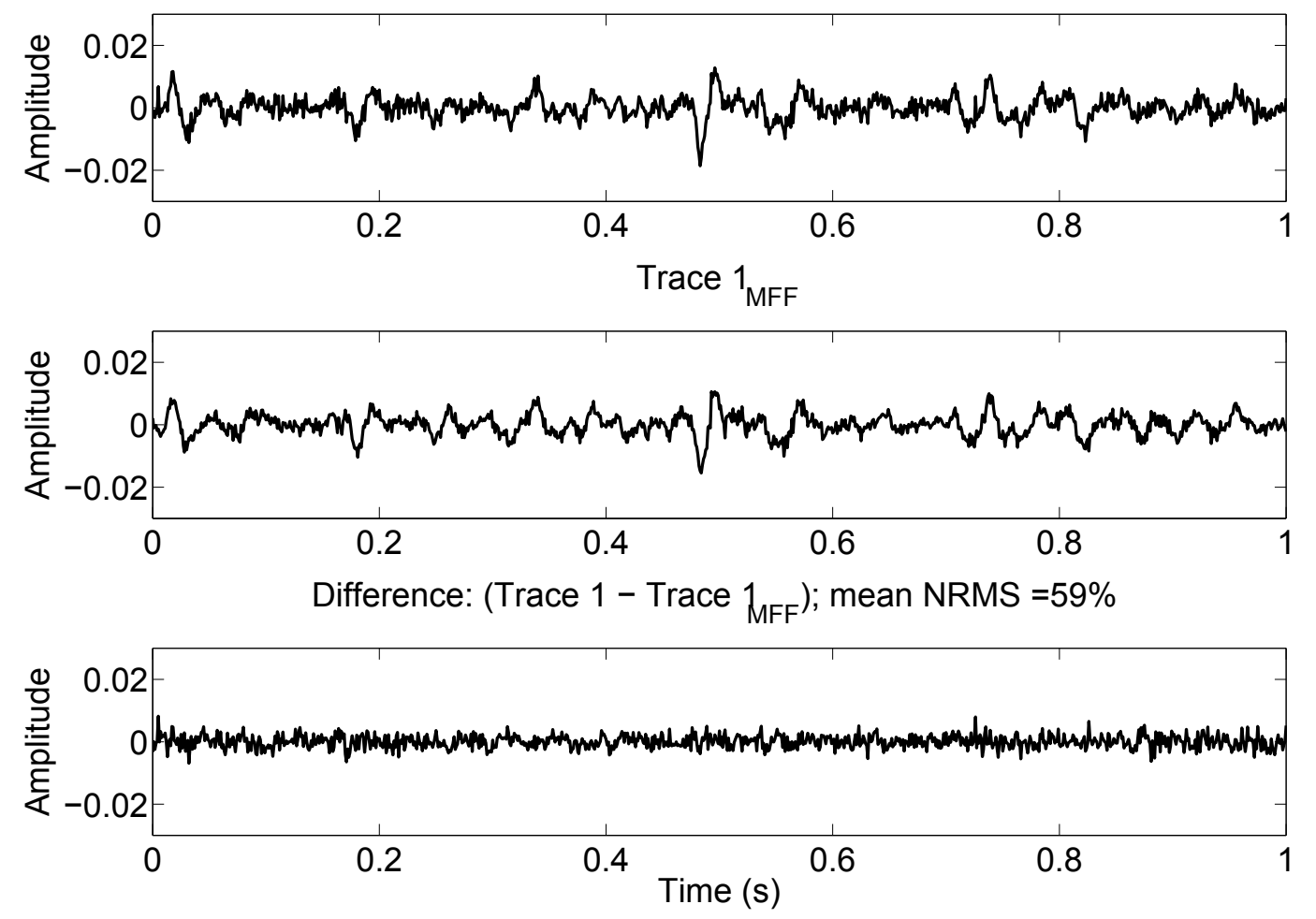

Figure 3.7: The spectral ratio match filtering of Trace 2 to Trace 1 where the result is Trace $1_{M F F}$. 


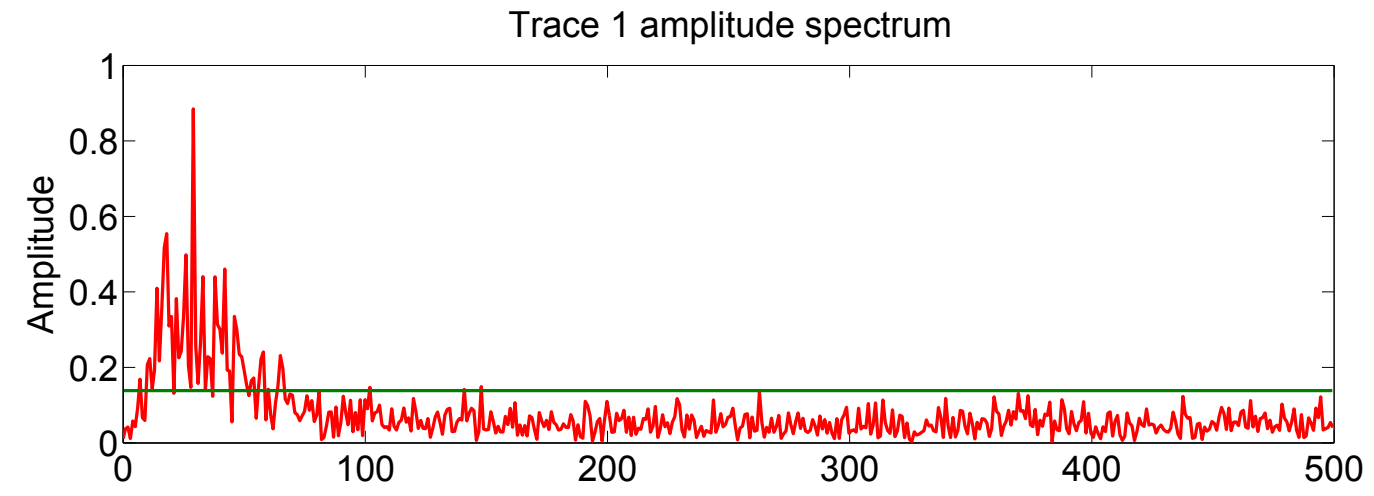

Trace 2 amplitude spectrum

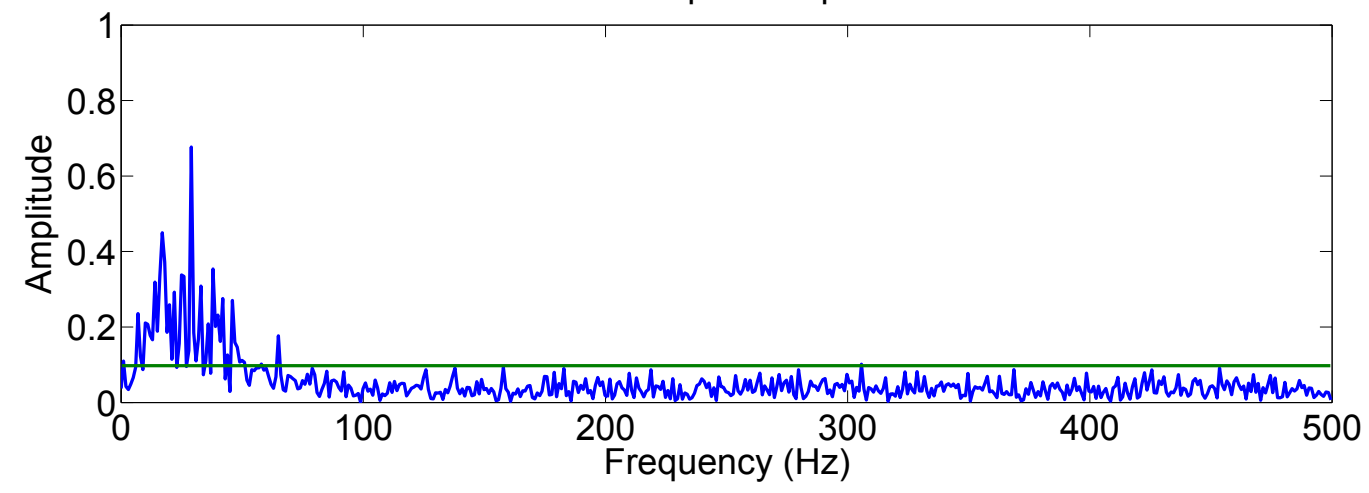

Figure 3.8: Amplitude spectrum of Trace 1 (top) and Trace 2 (bottom) from Figure 3.5. Note that the signal bandwidth is different for both traces (even after bandlimiting the traces, difference will still exist). The horizontal lines define the approximate noise level of the spectrum. 


\subsubsection{SCMF: time-domain LSQ vs spectral ratio}

Equation 3.12 is exact, and the repeatable parts of $d_{1}$ and $d_{2}$ will cancel out (i.e. reflectivity in the midpoint term). However, the spectral division renders it unstable if the data are band-limited or if noise dominates. In this thesis, we solve the time-domain leastsquares (LSQ) system described by equation 3.11, then transform the solution to the frequency domain which is an alternative to equation 3.12. The advantage of the timedomain solution is that the match filter, and hence the spectral ratio, are constrained by the higher amplitude time-domain components of the signals. The alternative is to directly solve equation 3.12 by multiplying the numerator and denominator by the con-

jugate transpose of $\widehat{d}_{2}(\omega)$ and stabilize the division by adding a small positive constant to the denominator. The magnitude of this small constant is dependent on the signal bandwidth which is expected to vary throughout the dataset. We prefer solving the system in the time-domain since such detailed knowledge of the bandwidth is not required. The time-domain solution is stabilized by setting a singular value threshold determined by a few tests (i.e a number higher than the mean minimum singular values of few shot records). Choosing an optimum filter length is also important as the objective is not to over fit both signal and noise present in both traces. Therefore, a filter length that is considerably shorter than the input trace length is found to be a good choice.

Now that we have shown that a matching filter in the time domain is equivalent to a spectral ratio in frequency domain, we can elaborate more on equation 3.3. The left-hand side of equation 3.3 is a trace-by-trace matching filter computed in the time domain over a time window. We crosscorrelate and align both traces then compute a least-squares match filter which minimizes the difference between the aligned signals. This time-domain match filter is transformed to the frequency domain. The complexvalued surface-consistent system is solved independently for each frequency (Figure 3.9), followed by inverse Fourier transform of the solution back to time domain. By deriving 
our match filter after trace alignment, we hope to minimize difficulties caused by phase wrapping (wrapped phase is constrained to its principal value, $(-\pi,+\pi)$, otherwise it is called unwrapped phase (Poggiagliolmi et al., 1982)), or equivalently the multivalued nature of the complex logarithm. The phase of the final solution has two parts: 1) a linear phase corresponding to the time-shifts required to align the traces before the match filters are computed, and 2) the mixed phase of the actual least-squares match filters. The latter is expected to be small because of the trace alignment. In a parallel computation, we decomposed the trace-to-trace time shifts into a surface-consistent set. The output of the algorithm is a set of surface-consistent convolutional filters with associated surfaceconsistent time shifts and these filters and shifts will be used to match the monitor survey to the baseline survey in the least squares sense.

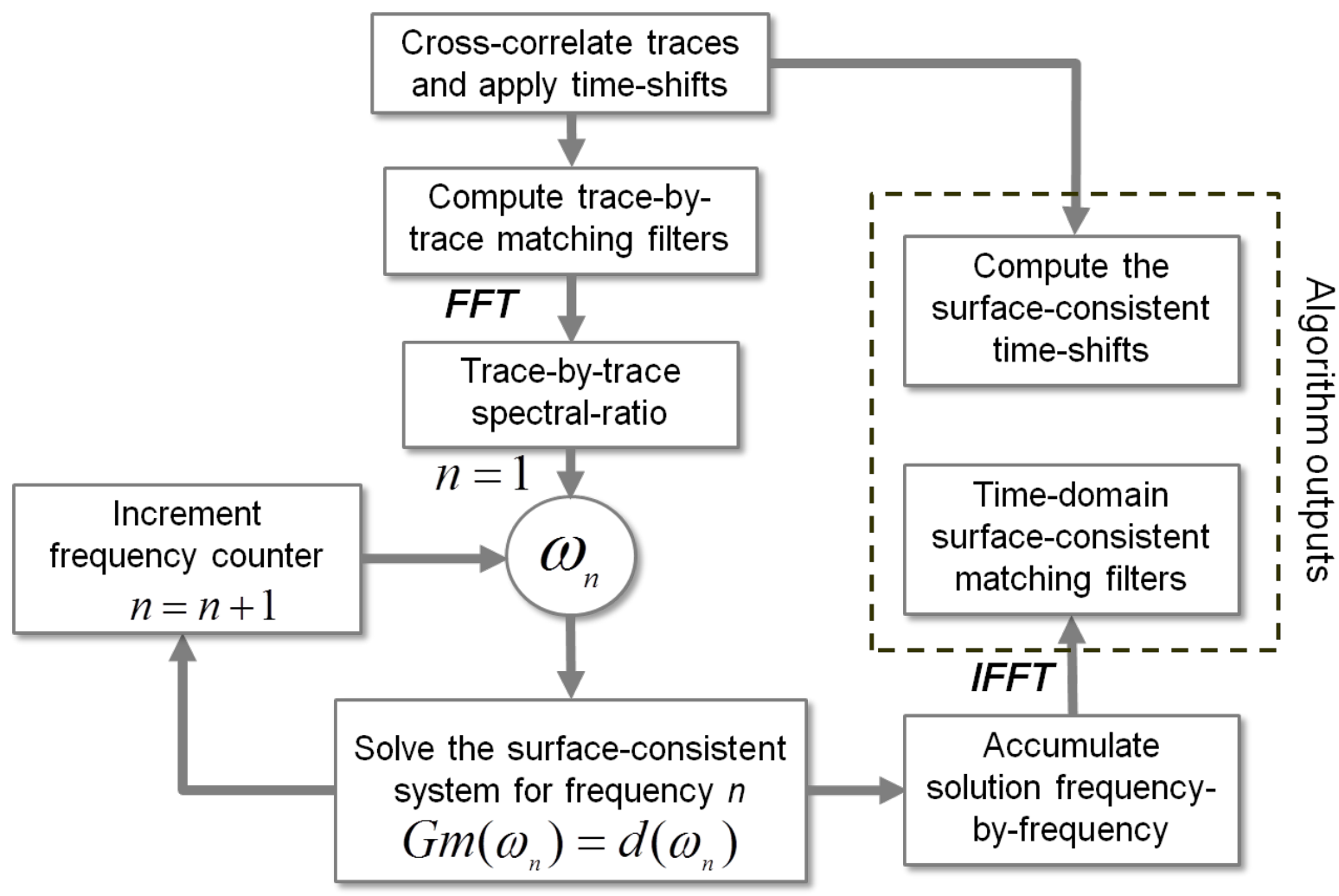

Figure 3.9: Processing workflow for the trace-by-trace surface-consistent matching filters. 


\subsection{Surface-consistent matching filter decomposition}

After solving the time-domain least-squares matching filter in equation 3.10 for each pair of traces in the two surveys and transforming the result to the frequency domain, we now consider a second least-squares decomposition of the solution into its four-component surface-consistent terms. This can be formulated as a general linear inverse problem (Wiggins et al., 1976) such that

$$
\operatorname{Gm}(\omega)=\mathbf{d}(\omega)
$$

where $\mathbf{G}$ represents the seismic geometry matrix (Figure 3.10), and $\mathbf{m}$ is a vector of surface-consistent terms (the unknowns) shown in the right-hand side of equation 3.3. $\mathbf{d}(\omega)$ is a vector of the data log spectral ratios represented by the left-hand side of equation 3.3. Usually the number of columns of $\mathbf{G}$ equals the total number of sources $\left(N_{s}\right)+$ total number of unique receivers $\left(N_{r}\right)+$ total number of offset bins $\left(N_{h}\right)+$ total number of midpoint bins $\left(N_{y}\right)$. Similarly, the number of rows of $\mathbf{G}$ equals the total number of traces ( $N_{t}=N_{s} N_{r}$, assuming each shot to have the same number of receivers), which is equal to the length of $\mathbf{d}$. The number of columns of $\mathbf{G}$ can be reduced by solving for only selected midpoints and offsets and interpolating between solution points. In this paper, we solve for all midpoints and offsets.

For all but the most trivial geometries, the problem is overdetermined (more equations, $N_{t}$, than unknowns, $N_{s}+N_{r}+N_{h}+N_{y}$ ). This is similar to the classic residual statics estimation problem (Wiggins et al., 1976). The problem is also known to be underconstrained (deficient in the number of independent equations available to solve for the unknowns). Wiggins et al. (1976) showed that the residual statics problem, which is essentially similar to this one, does not constrain "long wavelength" solutions. Mathematically, this is because $\mathbf{G}^{T} \mathbf{G}$ has a non-trivial null space, or equivalently, $\mathbf{G}$ has some zero singular values (Aster et al., 2005). $\mathbf{G}^{T}$ means the transpose of a real valued $\mathbf{G}$ or 


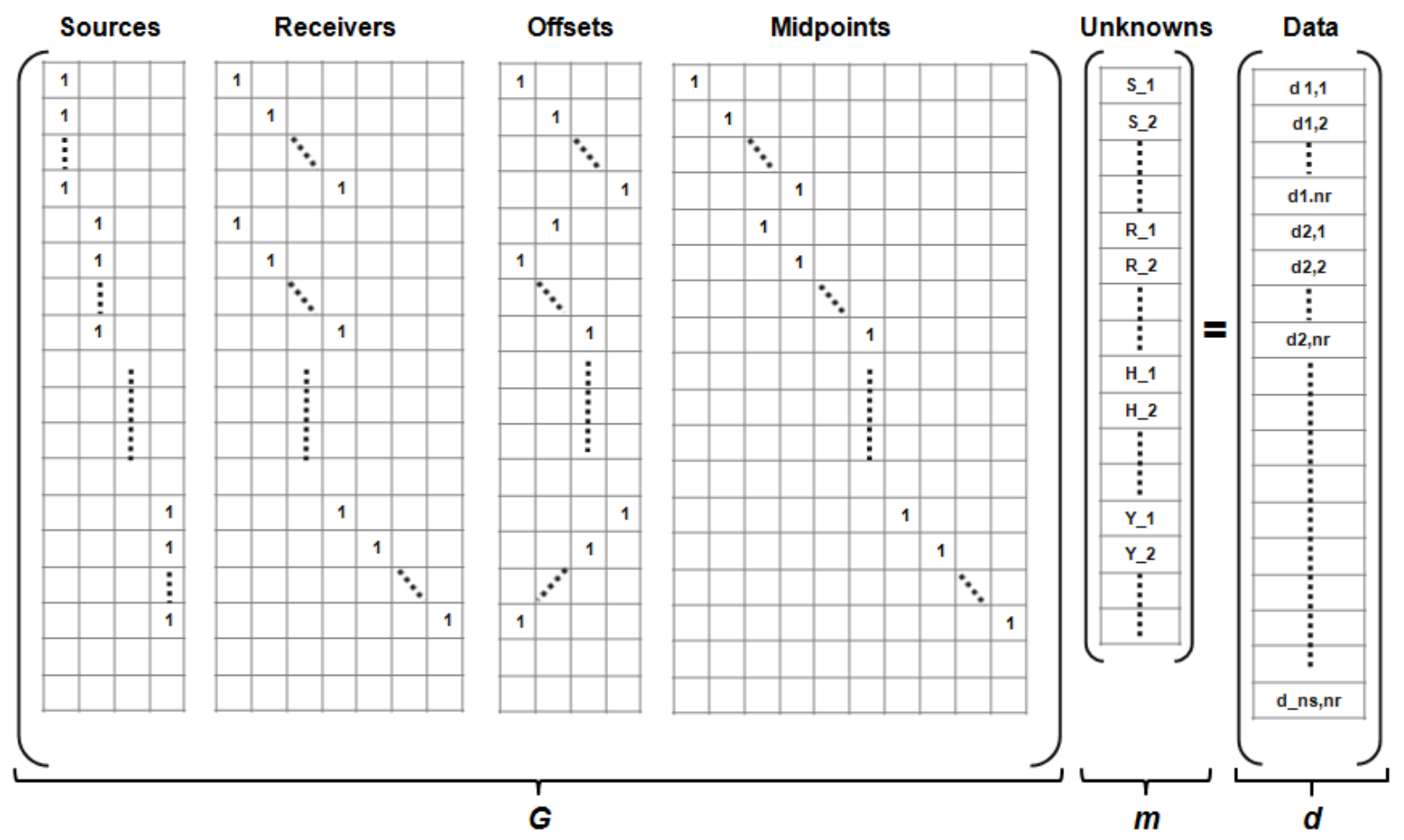

Figure 3.10: Matrix structure of the system of linear equation described in equation 3.13 ; the number of columns of $\mathbf{G}=$ number of sources + number of unique receivers + number of unique offsets + number of midpoints, and the number of rows of $\mathbf{G}=$ total number of traces; the length of $\mathbf{d}=$ total number of traces. 
the conjugate transpose if $\mathbf{G}$ is complex valued.

If $\mathbf{m}^{+}$represents a vector of the four unknowns, then the result of the least-squares decomposition is:

$$
\mathbf{m}^{+}=\left(\mathbf{G}^{T} \mathbf{G}+\alpha^{2} \mathbf{I}\right)^{-1} \mathbf{G}^{T} \mathbf{d}
$$

where $\alpha$ is a regularization parameter (a small positive number that can be estimated using the L-curve method) (Aster et al., 2005), and $\mathbf{I}$ is the identity matrix. Note that the matrix $\mathbf{G}^{T} \mathbf{G}+\alpha^{2} \mathbf{I}$ is nonsingular since $\alpha^{2} \mathbf{I}$ was chosen to constrain the null space of G. This method works well for a small data set like our example. The inversion method we used to solve equation 3.14 is the damped least-squares method, as properly adapted for complex-valued data. The solution results in complex-valued filter coefficients determining both amplitude and phase for each frequency. However, with large data sets direct inversion of the system in equation 3.14 will be expensive, and usually iterative methods such as conjugate gradient or Gauss-Seidel are utilized (Morley and Claerbout, 1983; Wiggins et al., 1976; Cary and Lorentz, 1993). When using iterative methods for decomposing the surface-consistent terms, it is common to compute a line-average spectrum from all traces in the data at first, then treat the four surface-consistent terms as residuals with respect to this line-average term. We did not attempt to separate the line-average spectrum since we are applying all four surface-consistent terms, but it will be a subject of a future investigation.

\subsection{Summary}

We have developed a method to design surface-consistent matching filters that can be used to match one dataset to another in a time-lapse experiment. The new algorithm is similar to the well known surface-consistent deconvolution except that the data required are the trace-by-trace spectral ratios of two datasets instead of the spectrum of a single 
one. We have demonstrated that computing the spectral ratios in a stable manner is possible by Fourier transforming the trace-sequential time-domain least-squares matching filters. A subsequent least-squares solution then factors the trace-sequential matching filters into four surface-consistent operators: source, receiver, offset, and midpoint. The output of the algorithm is a set of surface-consistent convolutional filters with associated surface-consistent time shifts. These filters and time shifts will be used to match the monitor survey to the baseline survey in the least squares sense. 


\section{Chapter 4}

\section{SCMF: application to a time-lapse model experiment}

\subsection{Introduction}

It is common to have unavoidable differences in acquisition geometry between repeated seismic surveys, differences in the source waveforms, differences in receiver responses or even differences not related to equipment, such as near-surface conditions (wet versus dry near-surface, tides, winds, etc.) (Beasley et al., 1997; Ross and Altan, 1997; Rennie et al., 1997; Porter-Hirsche and Hirsche, 1998). Other differences can arise from inconsistent processing of time-lapse data due to different processing software or personnel, advances in processing technologies, etc. (Jack, 1998).

A known process that reduces time-lapse nonrepeatability, which we term 'repeatability noise', is cross-equalization (Ross et al., 1996), which is a poststack process of amplitude scaling, phase matching, and trace shifting designed over a temporal window where changes are not expected. Generally, this cross-equalization process reduces the nonrepeatablity effects in seismic acquisition and processing and produces a better timelapse image (Ross et al., 1996; Rickett and Lumley, 2001). Other time-lapse processing techniques are prestack "parallel" processing and "simultaneous" processing described by Lumley et al. (2003) which aim to reduce undesired time-lapse differences without match filtering. These are step-by-step time-lapse processing flows available in commercial software, and all of them, poststack and prestack, are collectively called cross-equalization (Lumley et al., 2003).

In Chapter 3, we proposed a new method, applicable to time-lapse surveys, that minimizes the difference between baseline and monitor surveys by deriving a set of surface- 
consistent, time-domain matching filters that, in a least-squares sense, match one survey to another. We defined the surface-consistent terms as filters that are separable into four components with spatial variation in source, receiver, midpoint, or offset coordinates. These filters are derived from the prestack data and explicitly model changes in source and receiver wavelets, near-surface traveltimes, and possible subsurface changes. We specifically target land acquisition where every effort has been made to re-occupy source and receiver locations. The seasonal variations of the near surface layer, from moist to frozen or water table fluctuations, directly cause source and receiver consistent changes while reservoir activity is primarily midpoint consistent. Offset consistent variations could result from either near-surface or subsurface changes. Thus our technique is targeted to land acquisition, and may not be optimal in other settings such as marine acquisition.

Lumley (2001) and Calvert (2005) highlighted techniques developed for processing time-lapse data including parallel and simultaneous processing. In a time-lapse processing sequence, baseline and monitor surveys can be processed separately with exactly the same processing steps and with the same parameters, and this is commonly known as parallel processing. Alternatively, simultaneous processing merges baseline and monitor surveys into a single dataset when computing static corrections, deconvolution, amplitude scaling and other surface-consistent processes. In this simultaneous processing, the data are merged by common midpoint (and optionally common offset), and source and receiver terms solved independently using the larger dataset. Simultaneous processing is often advocated for time-lapse processing since the long-wavelength error (embedded in the midpoint term), when correcting residual statics, is common to each survey and is easily suppressed (Calvert, 2005). In addition to the statics corrections and deconvolution, the velocity model should be jointly developed for both surveys. For land time-lapse data, both parallel and simultaneous processing address the source and receiver variability, 
but neither process attempts to directly match these terms. In the presence of noise, the individually deconvolved datasets may have quite dissimilar residual wavelets.

In this chapter, we will demonstrate that the proposed surface-consistent matching filters will resolve the aforementioned problem by matching the wavelets and the statics of one survey to the other. After matching both surveys surface-consistently, we process them simultaneously but with a slight modification so that the merged data share a common source, a common receiver, a common offset and a common midpoint solution for every co-located term in every surface-consistent process. This type of time-lapse simultaneous processing is clearly surface- and subsurface-consistent while the first one described is only subsurface-consistent simultaneous processing.

To put our new algorithm in context with these techniques, we consider two seismic lines, baseline and monitor, acquired with exactly the same surface acquisition geometry at two different calendar times. The source and receiver wavelets for the two lines are different due to seasonal changes in near-surface. Event times will also be different as the traveltimes through the near surface layer will vary seasonally, that is, the statics solutions will depend on the season. The goal is to detect and minimize the nonrepeatable differences between the two lines. We list four different scenarios to address this simple example:

1. Independent or parallel surface-consistent processing without matching filters: This approach will estimate source and receiver wavelets and the statics solutions independently for each line. In principle, the resulting deconvolutions may result in both datasets having the same wavelet but this is not guaranteed as seasonal variations and noise may cause problems. Similarly, it is possible that the independent statics solutions could account for seasonal variations but again noise might make this unlikely. Also, the aforementioned long-wavelength error common to surface-consistent meth- 
ods is a problem for both deconvolution and statics.

2. Simultaneous surface-consistent processing without matching filters: This approach will produce an independent wavelet estimate for each source and each receiver in both surveys, and the resulting deconvolution operators must be applied to both lines. The estimated source and receiver wavelets should be similar to those estimated by parallel processing but the advantage is that the long-wavelength error is guaranteed to be common in both surveys.

3. Surface-consistent matching filters followed by independent surface-consistent processing: The effect of surface-consistent matching filters will be to match the source and receiver wavelets in one survey to the other. The nearsurface traveltime differences are also matched. Then, independent processing may possibly succeed in deconvolving these wavelets and removing the near-surface traveltime variations. However, since both lines will still have unique noise, it is unlikely that identical deconvolution operators and statics will be estimated.

4. Surface-consistent match filtering followed by simultaneous surface-consistent processing: As in scenario 3, the surface consistent matching filters should equalize the source and receiver wavelets and the statics in the two lines. This is followed by surface-consistent simultaneous processing (i.e scaling, deconvolution and static correction) which is generalized to apply the same source and receiver deconvolution operators to each line and the same statics solution. Additionally any long-wavelength error in the solution is common and vanishes when taking the difference.

The above four approaches are used as a thought experiment to motivate our focus of 
attention on the last method. It is a common approach, as highlighted by Calvert (2005), to start the time-lapse processing with trace equalization in order to reduce trace-totrace variations prior to merging both surveys for the simultaneous processing. However, to better constrain our processing sequence and remain surface-consistent, we suggest employing surface-consistent matching filters after coherent noise attenuation but prior to any other surface-consistent processing.

In the following section, we present an analysis of a modeled time-lapse synthetic data set that has many of the complexities generally observed in a real data set. Through this 2D example, we will calculate the SCMF, analyze its components, and measure the time-lapse repeatability after apply these filters. Here, we will only focus on the new developments of this thesis, that is the modified simultaneous processing, where all four terms are consistent after applying the SCMF. In Chapter 5, we will expand our analysis so that it includes a parallel processing comparison of a real time-lapse dataset example.

\subsection{Time-lapse model experiment}

For this experiment, we constructed a simple $2.5 \mathrm{~km}$ wide and $1 \mathrm{~km}$ thick $2 \mathrm{D}$ model. The model consists of five layers including a reservoir unit, $500 \mathrm{~m}$ wide and $20 \mathrm{~m}$ thick , between layers three and four. The velocity is homogeneous in each layer, except for the near-surface layer, where random lateral variations were introduced. Using this geometry, we generated two earth models, a baseline model and a monitoring model (Figures 4.1a and 4.1b), with different near-surface and reservoir velocities (Figures 4.1c). afd_vmodel from the CREWES Matlab toolbox was used to generate these models. Velocity variation in the near-surface due to seasonal soil variations is one of the nonrepeatability issues for land time-lapse data. In Canada, such seasonal variations can be extreme between winter and summer, and can also involve variations in near-surface attenuation. Our 
baseline model had a slower near-surface simulating summer acquisition while our monitor model had faster velocities simulating winter acquisition. The ratio of the baseline and the monitor near-surface velocity models is a constant scale factor. We also included a variable Q (Figure 4.2a) in the near-surface layer, which effectively means that the source wavelet and the receiver response show variable bandwidth laterally along the line. The einar function of the CREWES Matlab toolbox was used to generate these filters.

An acoustic finite-difference modeling algorithm, afd_shotrec_alt, was used to acquire 51 shot records at $50 \mathrm{~m}$ shot interval using a receiver array of 101 geophones at $10 \mathrm{~m}$ spacing. We used the 2D split-spread acquisition geometry and shot through the tail spread on each end. The maximum record length is $1 \mathrm{~s}$ with a $4 \mathrm{~ms}$ sampling interval. A minimum phase wavelet with $20 \mathrm{~Hz}$ dominant frequency was created to simulate the impulse sources. We used a $1 \mathrm{~m}$ bin spacing for both horizontal and vertical, and a 0.0001 $\mathrm{s}$ time step for the modeling. We also used the $2^{\text {nd }}$ order or the 5 points approximation to the laplacian operator. Each shot record and each receiver record was scaled by randomly generated scalars (Figures $4.2 \mathrm{~b}$ and $4.2 \mathrm{c}$ ) to simulate variations in source strength and receiver coupling. Hence, any implied changes to the shapes of the source and receiver wavelets is due solely to variations in $\mathrm{Q}$.

Note that our monitor survey has identical acquisition geometry to the baseline survey. For land acquisition, this is a likely scenario given accurate GPS locations. Even in the presence of drilling or production installations or possible failure of some sources or receivers, we think that only closely repeated sources and receivers should be selected from both surveys. In other words, if a source record is missing in the monitor survey, then the corresponding one in the baseline should be removed and vice versa. Calvert (2005) made a similar argument about positioning and data selection and that the gain of this is higher, particulary in our effort to suppress repeated noise.

Raw shot records from the baseline and the monitor surveys are shown in Figures 


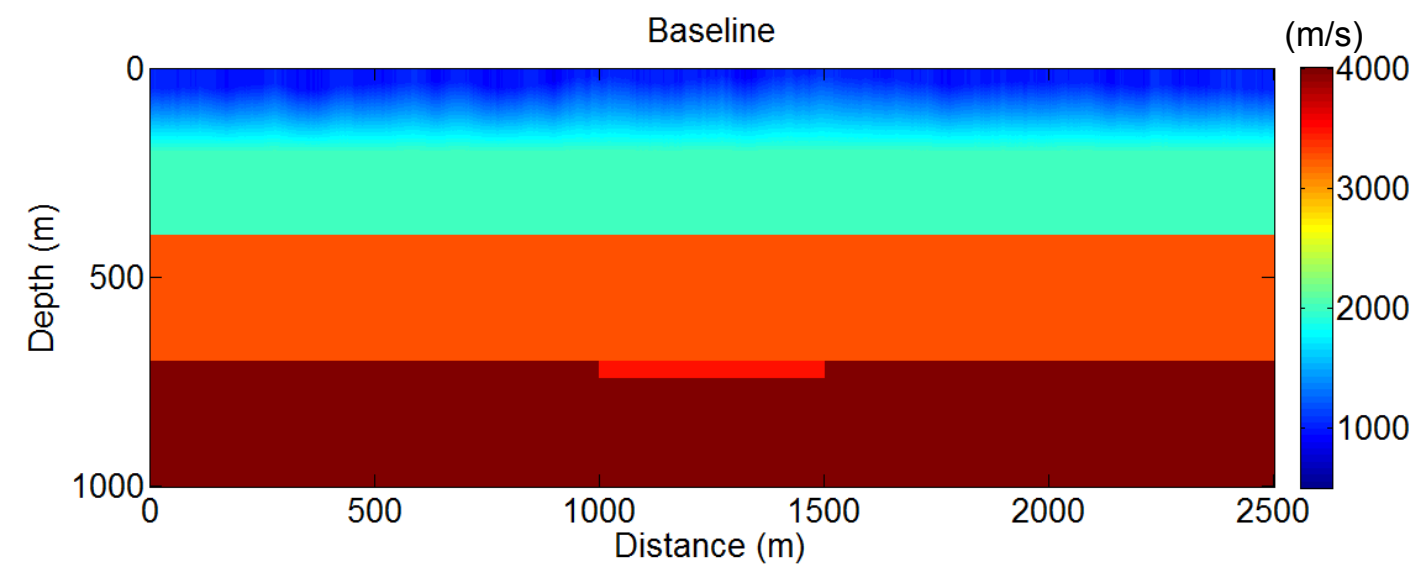

(a)

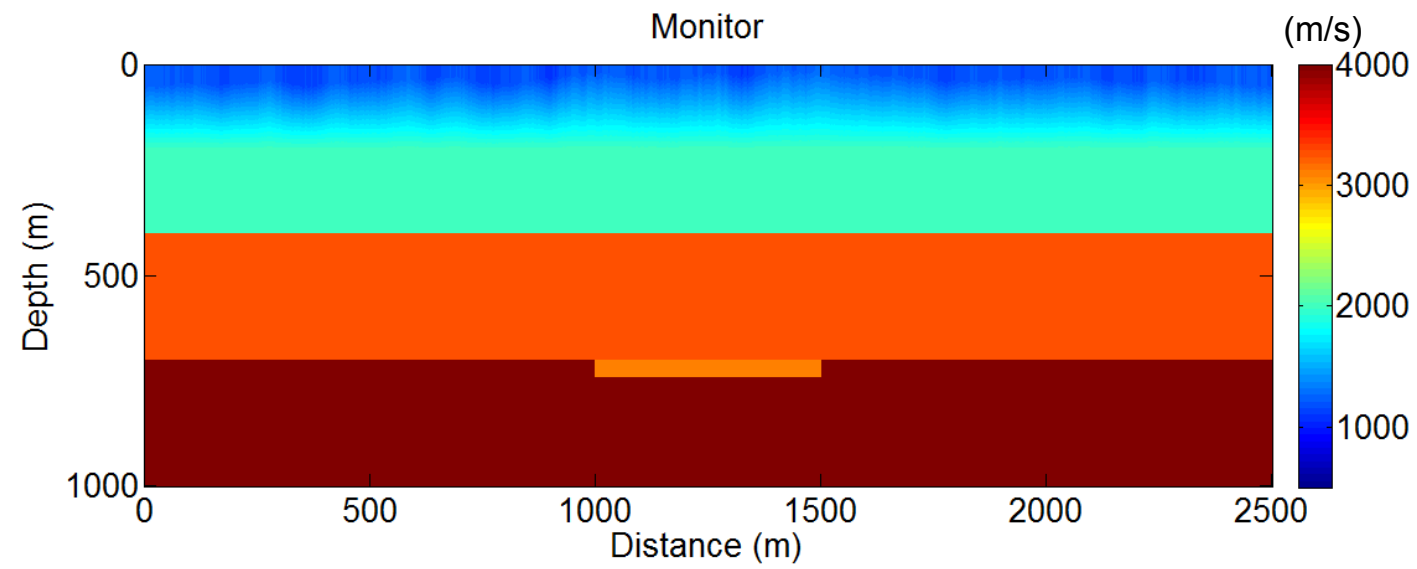

(b)

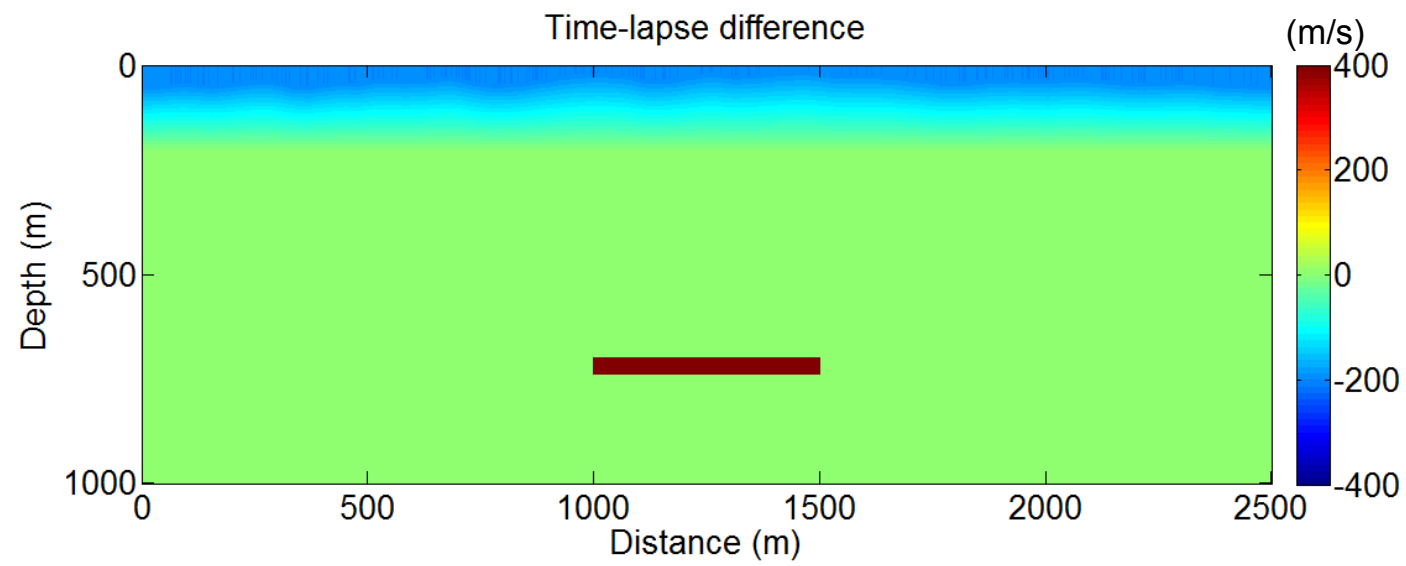

(c)

Figure 4.1: Two earth velocity models (units in $\mathrm{m} / \mathrm{s}$ ) representing baseline (a), monitor (b), and their time-lapse difference (c). Note that both models have both near surface (showing effects of dry vs wet climate) and in the reservoir unit. 

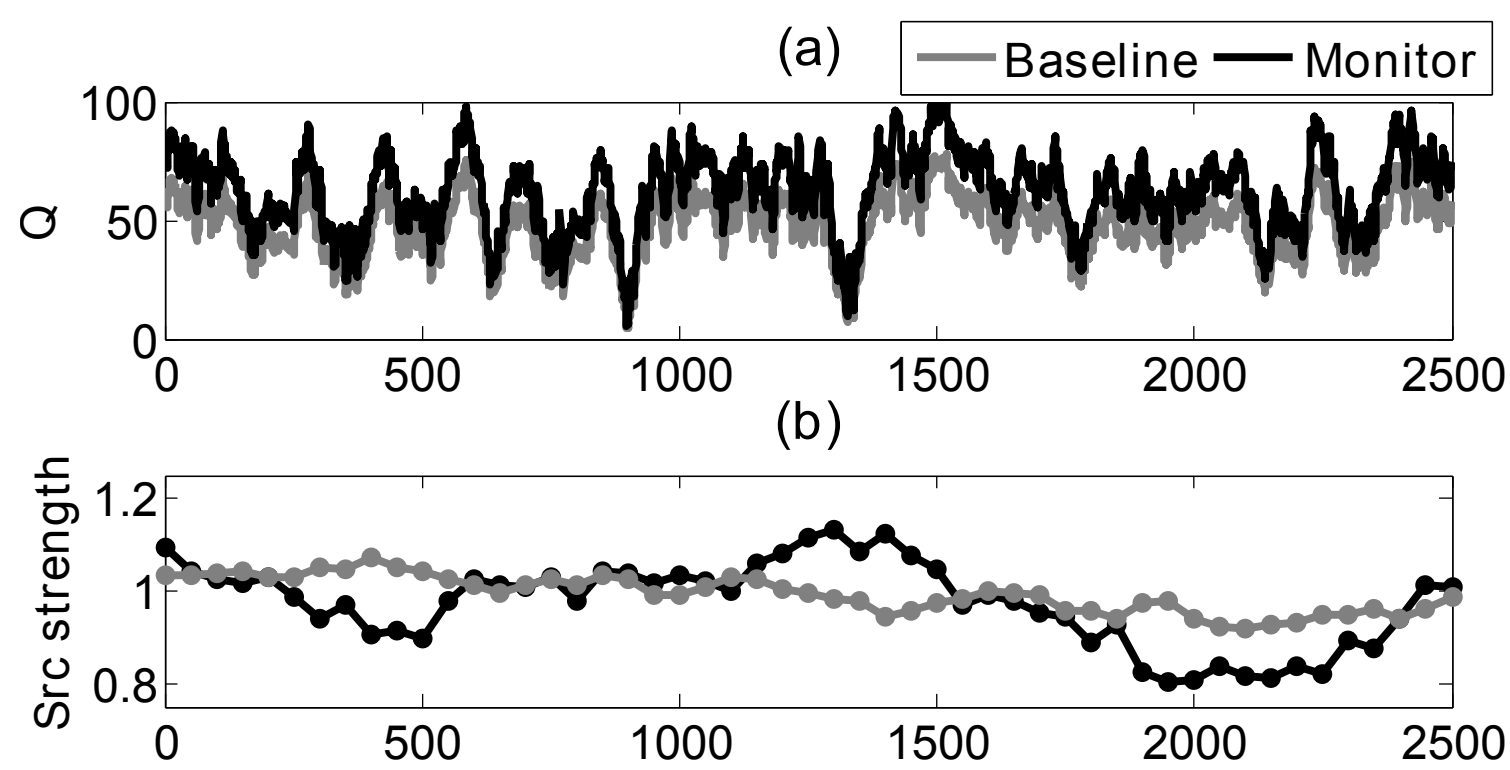

(c)

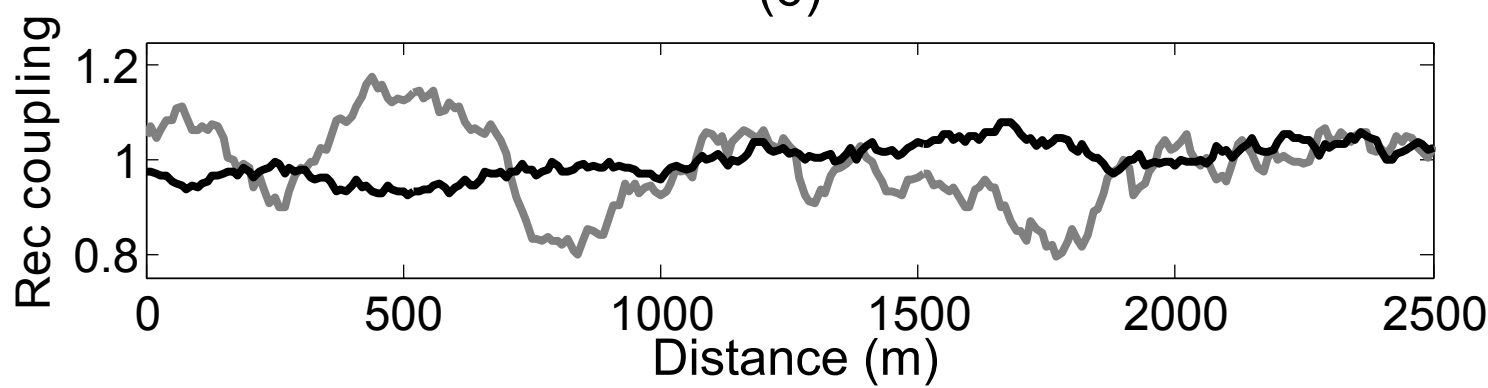

Figure 4.2: Horizontal profile of near-surface attenuation for baseline model (gray line) and monitor model (black line)(a), source strength (b), and receiver coupling (c). 
4.3a and $4.3 \mathrm{~b}$. After muting the first breaks, Figures $4.4 \mathrm{a}$ and $4.4 \mathrm{~b}$, respectively, there is a large difference between these two unprocessed shot records, Figure 4.4c, particularly in arrival time of events, amplitude and phase. Note that in these plots black color represents peaks and white represents troughs and all plots have the same amplitude clipping as that of the baseline seismic. A commonly used metric that measures the similarities between traces is called NRMS (normalized root mean square error) Kragh and Christie (2002). We computed the NRMS inside a window of approximately $300 \mathrm{~ms}$ just above the reservoir unit $(\sim 0.75 \mathrm{~s}$ in the middle of the shot record indicated by two dashed lines), and the result is shown in Figure $4.4 \mathrm{~d}$ with a mean of $144.7 \%$. NRMS is computed using the following relationship discussed in Kragh and Christie (2002):

$$
N R M S(\%)=200 \times \frac{R M S(a-b)}{R M S(a)+R M S(b)},
$$

where, for example, the

$$
R M S(a)=\sqrt{\frac{1}{n}\left(a_{1}^{2}+a_{2}^{2}+\ldots+a_{n}^{2}\right)} .
$$

Theoretical NRMS values range from 0 to $200 \%$, where low values correspond to similar traces, and high values correspond to extremely dissimilar ones. One possible way to reduce the difference observed in Figure $4.4 \mathrm{c}$ is by adding a constant time shift to the monitor shot record. Figure $4.5 \mathrm{a}$ is the same baseline shot record shown earlier, and Figure $4.5 \mathrm{~b}$ is the same monitor shot record shown in Figure $4.4 \mathrm{~b}$ shifted down by $26 \mathrm{~ms}$ so that the middle reflector matches the same reflector on the baseline shot. Figures $4.5 \mathrm{c}$ and 4.5d illustrate their differences and their computed NRMS, respectively. Although the mean NRMS is reduced to $41.4 \%$ due to the added constant time shift, each monitor survey shot record requires a different time shift. Additionally, significant differences remain due to the mismatch in amplitude and phase.

Examining NRMS versus time shift of two noise-free signals (Figure 4.6a) shows a clear linear relationship (or $N R M S(\%) \approx 100(2 \pi f \delta t$ ), where $f$ is the dominant frequency and 
$\delta t$ is the time shift between the two traces) (Calvert, 2005). NRMS error versus amplitude difference (Figure 4.6b) is only linear for a small amplitude residual (or $N R M S(\%) \approx$ $100(\delta a)$, where $\delta a$ is amplitude residual between the two traces) (Calvert, 2005). The high NRMS values, ranging from $120 \%$ to $150 \%$ seen in Figure $4.4 \mathrm{~d}$, suggest a highly nonrepeatable time-lapse experiment, as we had intended our synthetic to be.

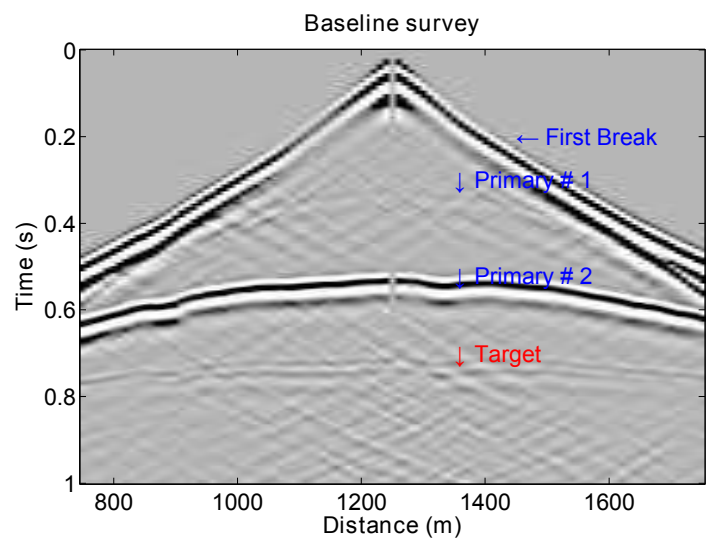

(a)

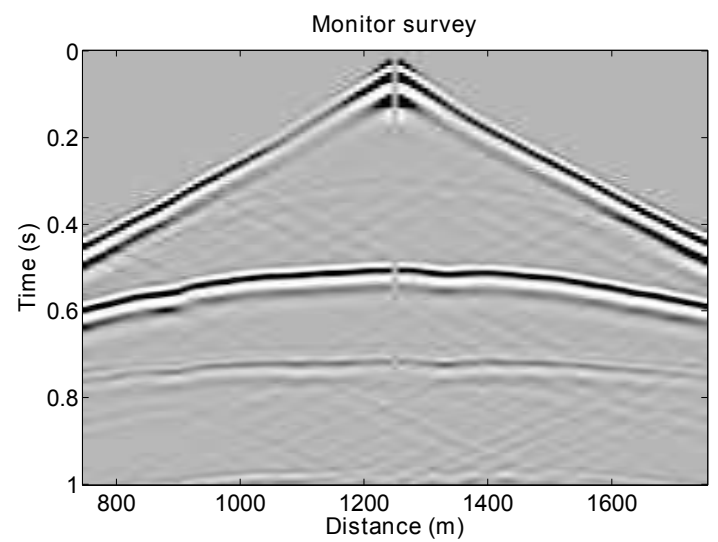

(b)

Figure 4.3: Two raw shot records, source location at $1250 \mathrm{~m}$, from (a) the baseline survey and (b) the monitor survey.

\subsubsection{Computing surface-consistent matching filters}

We have computed the four-component surface-consistent trace-by-trace matching filters inside a $300 \mathrm{~ms}$ window above the target (Figures $4.4 \mathrm{a}$ and $4.4 \mathrm{~b}$ ) for all 51 shot records. Using equation 3.14, we decomposed these trace-by-trace matching filters into a set of surface-consistent matching filters. There are similarities between the interpretation of the surface-consistent log spectral decomposition of the matching filters and that of the residual statics described by Wiggins et al. (1976). In the residual statics problem, contribution from the source and receiver terms as well as structure and residual normal moveout (NMO) were clear and reported by Taner et al. (1974). Similar distinctions were observed in the surface-consistent matching filters where each of the four terms collects 


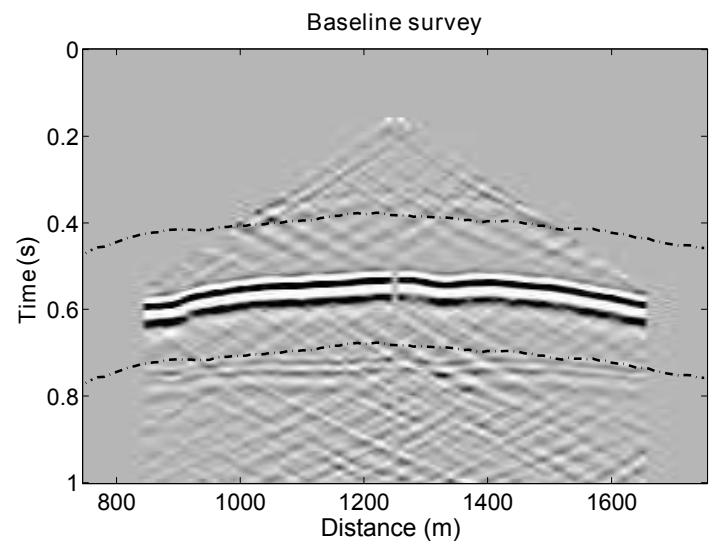

(a)

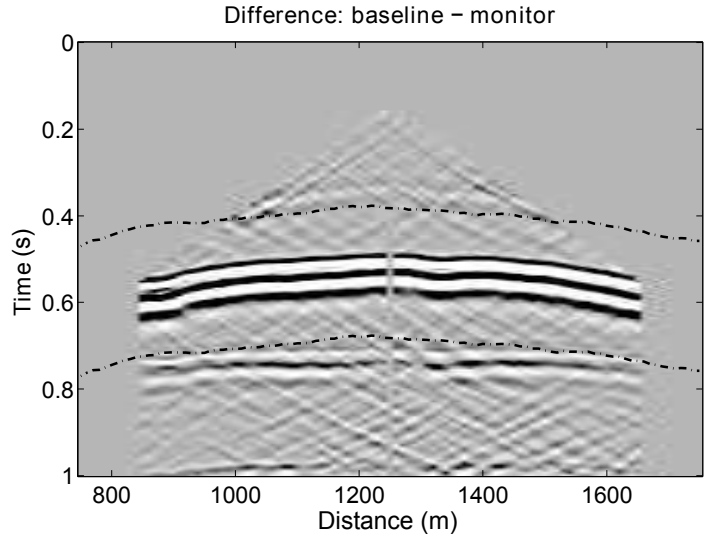

(c)

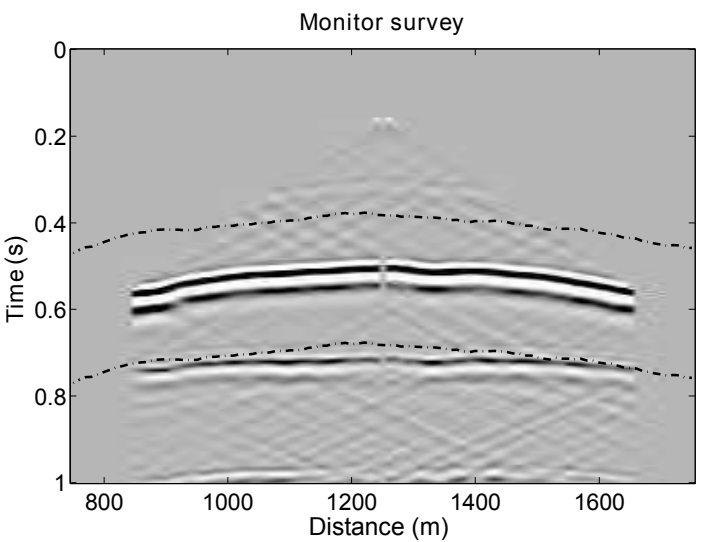

(b)

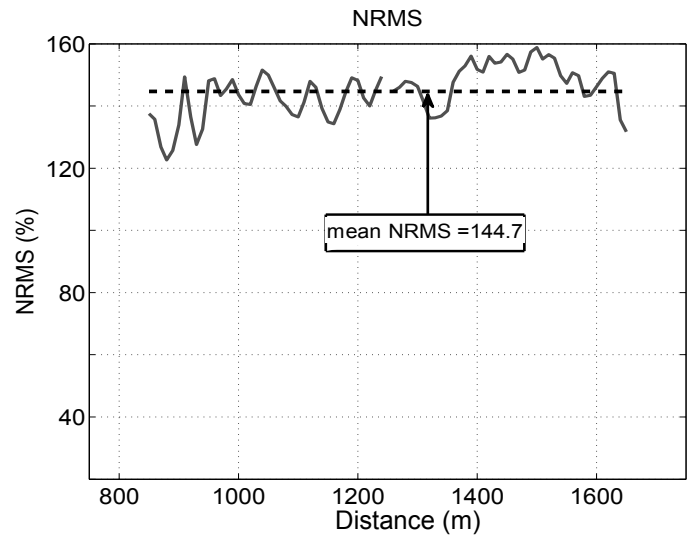

(d)

Figure 4.4: Two shot records, source location at 1250m, from the baseline survey (a) and the monitor survey (b). (c) shows the difference between (a) and (b), and (d) shows the NRMS computed between the two dotted lines (the analysis window). Note the mean NRMS is $144.7 \%$ which means both surveys are highly dissimilar. Black color on all seismic plots represents peaks and white represents troughs. All seismic displays have the same amplitude clipping as the baseline figure. 


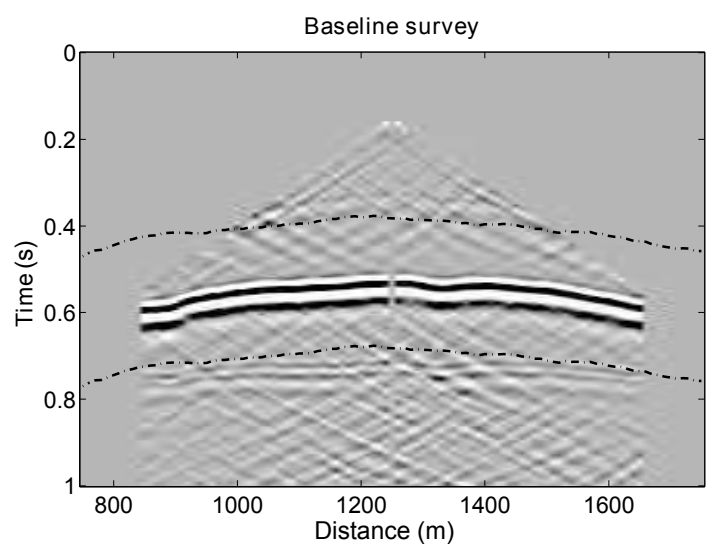

(a)

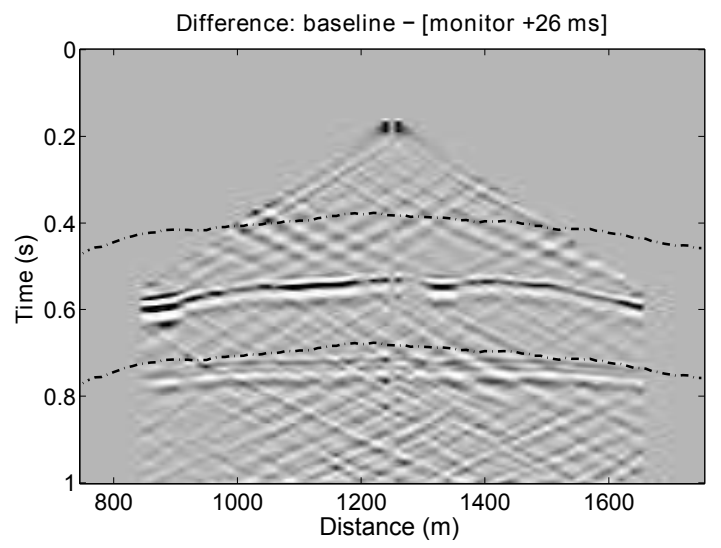

(c)

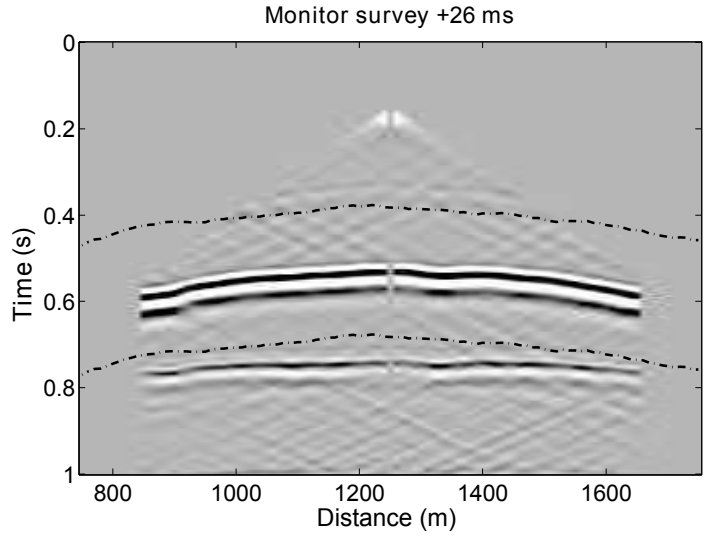

(b)

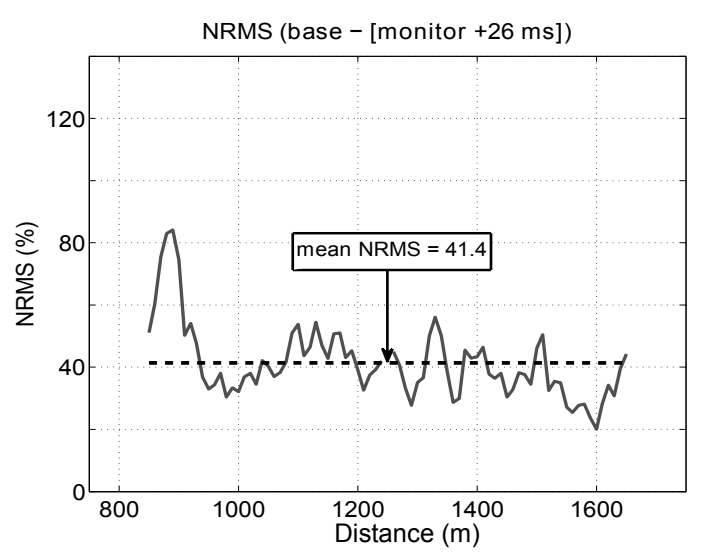

(d)

Figure 4.5: Baseline shot (a), similar to Figure 4.4a, and monitor shot with $+26 \mathrm{~ms}$ time shift (b). The difference of (a) and (b) is shown is (c), and (d) shows the NRMS computed between the two dotted lines (the analysis window) of the difference plot. Note that the mean NRMS has decreased to $41.4 \%$ but there is a significant amplitude difference. 


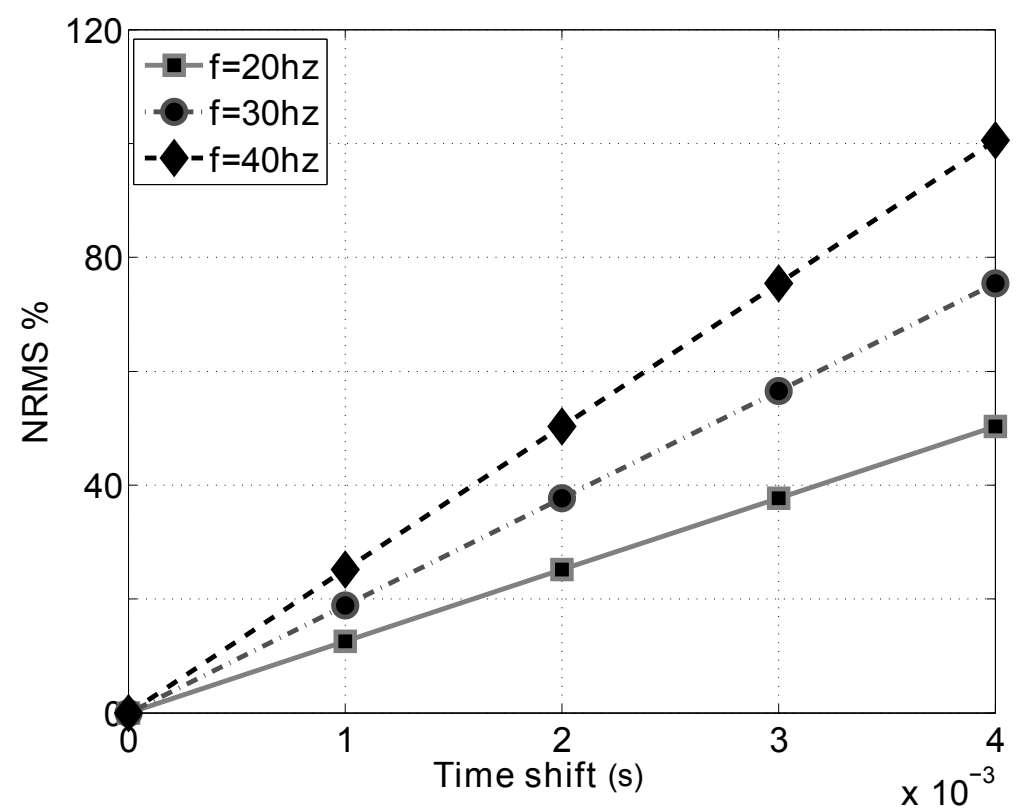

(a)

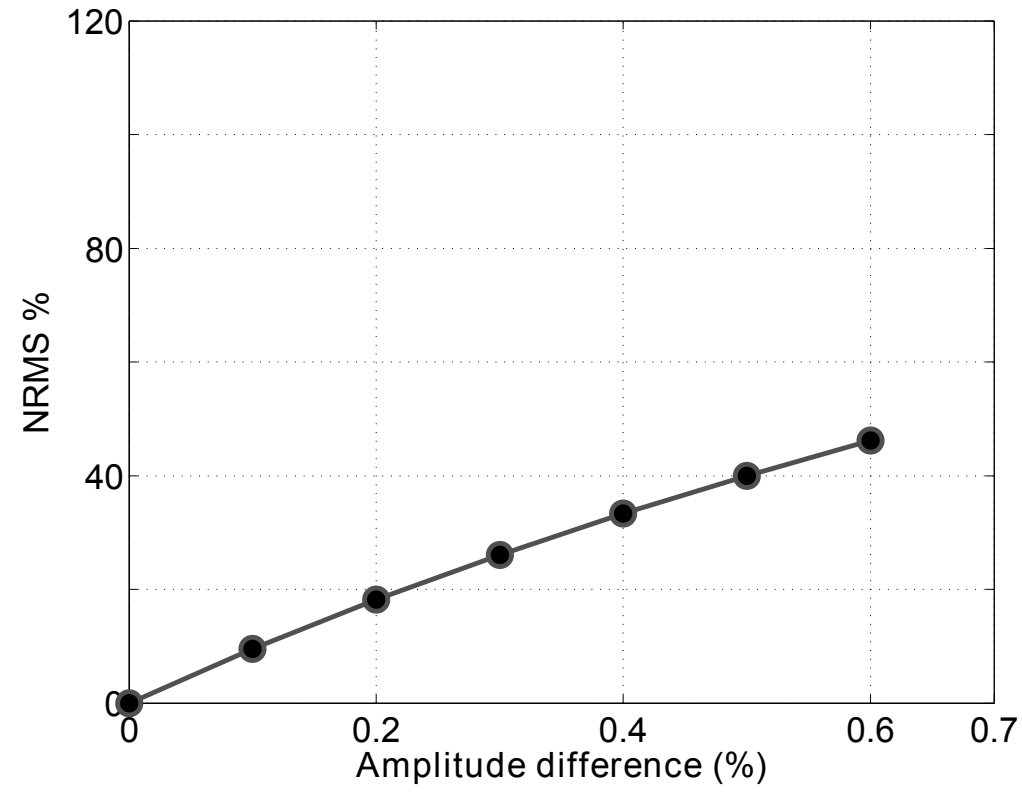

(b)

Figure 4.6: NRMS sensitivity tests where (a) is NRMS versus time shift for three dominant frequencies and (b) is NRMS versus amplitude difference between two noise free traces. 
different effects.

The source and receiver terms

Since we are decomposing a log spectral ratio of two data sets, we interpret the source term as a log spectral ratio of the two corresponding source signatures, which we take to include the actual source wavelets and local modifications caused by source strength variations and attenuation. After exponentiation and inverse Fourier transform, this yields a set of time-domain filters that match each source record in the monitor survey to the baseline survey. Similarly, the receiver term is a log spectral ratio of the two corresponding receivers' response and the near-surface effects around each receiver. When exponentiated and inverse Fourier transformed, a set of time-domain filters is obtained that matches each receiver in the monitor survey to the baseline survey.

Figures $4.7 \mathrm{a}$ and $4.7 \mathrm{c}$ show the source and receiver components computed from the raw shot records of the baseline and the monitor surveys. Figure $4.7 \mathrm{~b}$ illustrates the similarities between the mean (over frequency) shot filters (shown in Figure 4.7a), which we will refer to as source strength, and the ratio of baseline survey to monitor survey source strengths shown in Figure 4.2b. Similar effects can also be seen in the receiver components in Figure 4.7c where variations in receiver couplings are known (Figure 4.2c). Figure $4.7 \mathrm{~d}$ shows the similarities between the mean receiver filters (shown in Figure $4.7 \mathrm{c}$ ), which we will refer to as receiver strength, and the ratio of baseline survey to monitor survey receiver strengths. Separation of the source signature or the receiver impulse response from the surrounding near-surface geology effects is unnecessary, since we are primarily interested in applying these surface-consistent matching filters to the monitor survey (the survey in the denominator of equation 3.3) to reduce its mismatch with the baseline survey. 


\section{The offset and midpoint terms}

The two other components computed in the study are offset and midpoint terms. Figure 4.8a shows the log-amplitude spectrum of the offset term. Muting the first breaks was necessary due to their interference with reflections inside the analysis window that occurred at about offset $400 \mathrm{~m}$. Similar large amplitude estimates have been reported in the offset components by Cary and Lorentz (1993) and were attributed to coherent noise such as ground roll. In this study, after we muted the first breaks (Figures $4.4 \mathrm{a}$ and $4.4 \mathrm{~b}$ ), the newly computed offset terms were lower in amplitude than previously computed offset terms and smoother than the source and receiver terms. A smoother offset term is attributed to the fact that there are more traces contributing to each offset than to the source and receiver terms (Cary and Lorentz, 1993). Figure 4.8b represents the average over frequency of the offset filters, referred to as offset strength, shown in Figure 4.8a where amplitude values are high before offset number 40 but reduce to unity beyond, due to absence of data.

The midpoint filters in Figure 4.8c show rapid variations compared to the other components. Similar observations have been reported by Cary and Lorentz (1993) for the surface-consistent deconvolution problem. The midpoint term collects more noise than any other term because of the low fold (low number of traces per midpoint), but that can be minimized if smoothing is applied. In this study, the overall strength of the

midpoint filter (Figure 4.8d) is small (averaging about one), hence we decided not to apply smoothing.

\subsection{Filter application and analysis}

Applying the computed four-component surface-consistent matching filters in Figures 4.7a, 4.7c, 4.8a, and 4.8c to the monitor survey, we obtain a new survey that more 
closely matches the baseline survey. Figure $4.9 \mathrm{~b}$ illustrates a surface-consistent matched monitor survey that compares to the same shot record in the baseline survey (Figure 4.9a). The new difference is shown in Figure 4.9c. Note that the mismatch observed in Figure 4.4 has been reduced significantly, and that the computed NRMS in the window of analysis (between the two dashed lines) has reduced to a mean of $26.8 \%$.

In the next two sections, we will compare trace-by-trace and surface-consistent matching filters, before and after stack, and then examine the effects of the surface-consistent terms.

\subsubsection{Trace-by-trace (non-surface-consistent) and Surface-consistent matching filters}

The surface-consistent method separates the seismic trace into surface (source and receiver) and subsurface (offset and midpoint) components. Each of these four terms has

a different signature that can be examined and compared. In other words, traces that share the same source will always have the same source filter, and similarly those traces recorded by the same receiver will always have the same receiver filter. For comparison, we show results from application of trace-by-trace matching filters, computed prestack, which are used as input to our surface-consistent decomposition. The following two examples demonstrate this difference clearly. Figure 4.10a is the difference between a trace-by-trace matching filter applied to a single shot of the monitor survey and the baseline survey. In this example, the trace-by-trace matching filters reduced much of the mismatch between the two surveys and the mean NRMS is approximately $25 \%$. It is slightly less than that obtained by the four-component surface-consistent matching filters shown in Figure 4.9d. Figure 4.10b is the difference between the matched monitor survey stack and the baseline survey stack, for trace-by-trace matching filters. Before stacking, two successive iterations, with the same parameters and window length, of simultaneous surface-consistent residual static corrections were applied (using the stat function of the 


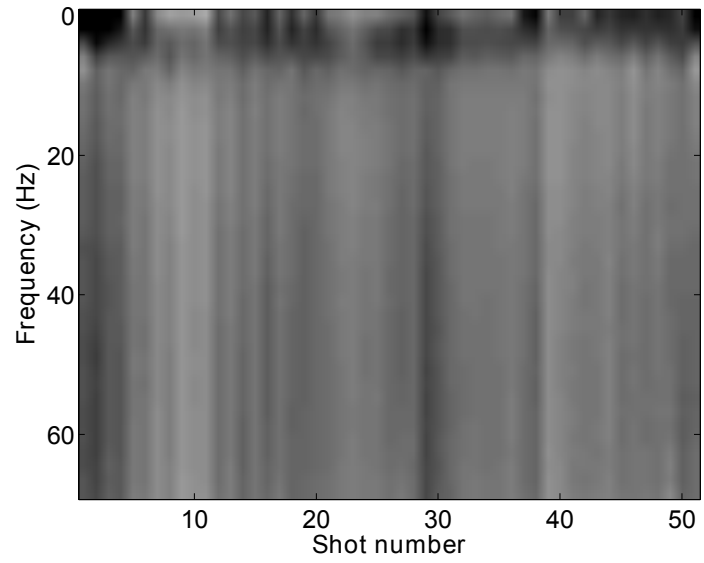

(a)

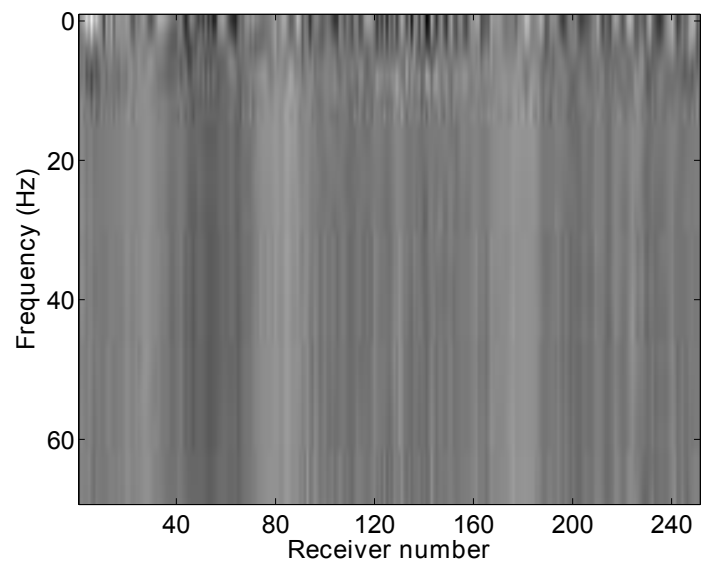

(c)

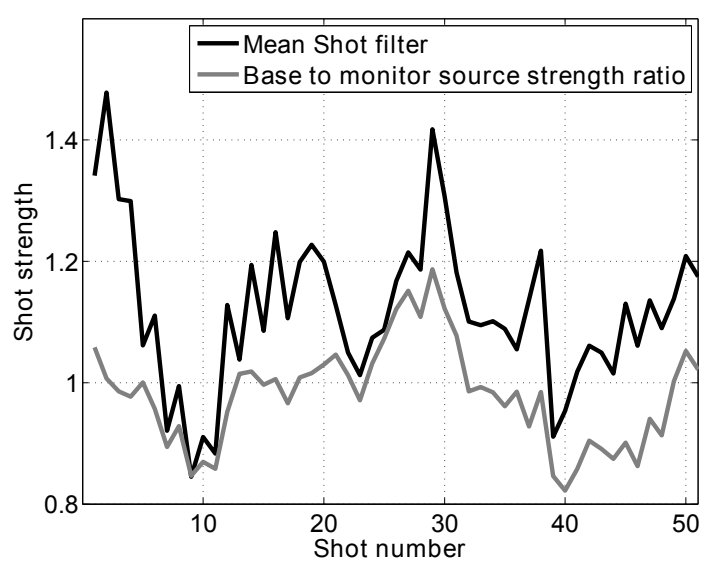

(b)

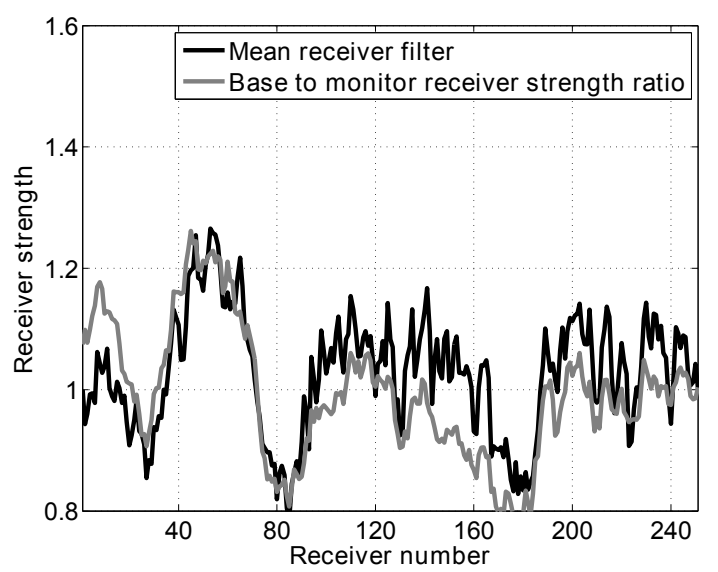

(d)

Figure 4.7: In (a) is the log amplitude spectra of the source filters computed using equation 3.14. In (b) is a comparison of the average over frequency of the shot filters, referred to as source filters strength, in black line versus the ratio of the baseline survey source strength to the monitor survey source strength in gray line. The log amplitude spectra of the receiver components is shown in (c) and in (d) is a comparison of the receiver filter strength (black line) versus the receiver strength ratio of both surveys (light gray line). 


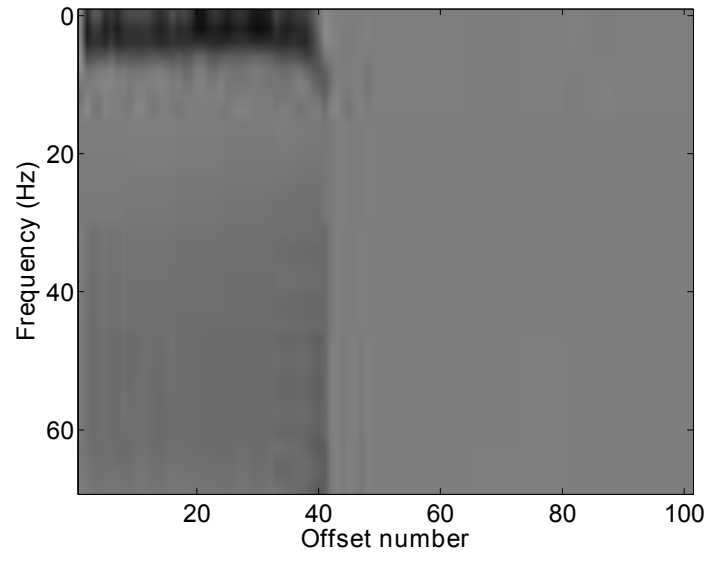

(a)

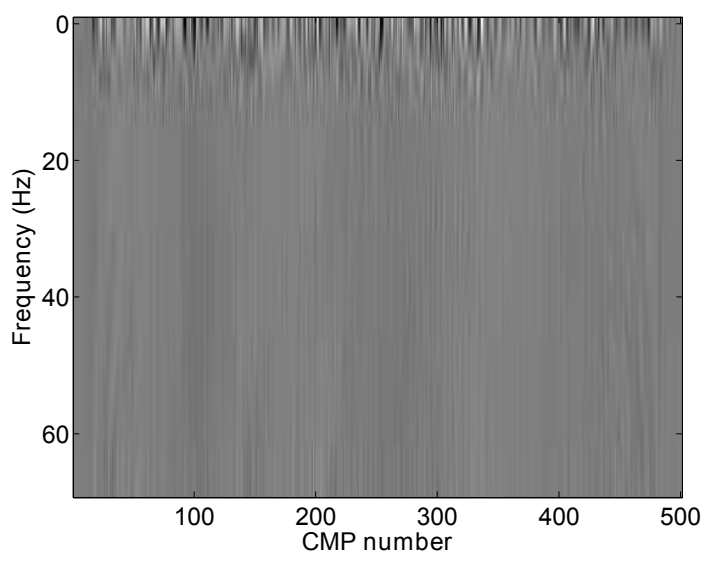

(c)

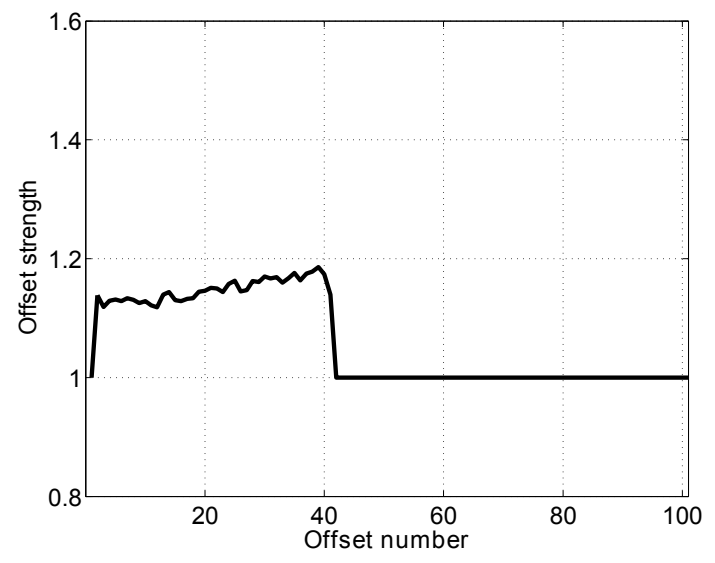

(b)

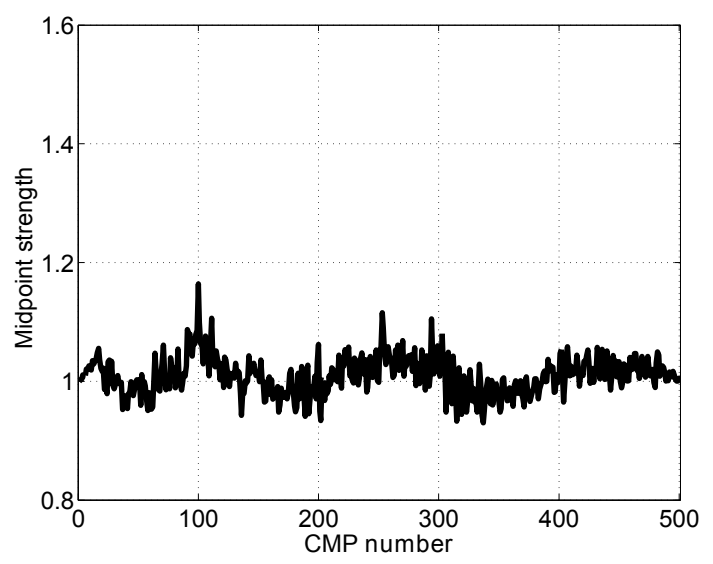

(d)

Figure 4.8: In (a) is the log amplitude spectra of the offset filters and in (b) is the offset filter strengths computed as the average over frequency. Similarly, in (c) is the log amplitude spectra of the midpoint filters and in (b) is the midpoint filter strengths computed as the average over frequency. 
CREWES Matlab toolbox). The mean NRMS computed in the filter window (between the two gray lines) in the figure is about 35\%. Comparing this result to that obtained from applying the four-component surface-consistent matching filters (Figure 4.11f), the latter one is preferred due to its low NRMS error of $15.7 \%$. The increase in NRMS after stacking of the trace-by-trace matching filters is due to the non-surface-consistency of those filters. This circumstance is more familiar in the context of residual statics where non-surfaceconsistent trim statics give superior CMP gathers but an inferior stack when compared with surface-consistent statics. In addition, the surface-consistent matching filters equalize both surveys, and if the filters are successful, then both surveys will have the same residual statics in them, in which case a simultaneous static correction is required. In summary, on a single shot record trace-by-trace matching filters produce a slightly lower NRMS (25\%) than surface-consistent matching filters (26.8\%). Also, after simultaneous surface-consistent residual static corrections then stacking, the surface-consistent matching filters produce a much better result compared to the trace-by-trace matching filters (NRMS is $15.7 \%$ versus $35 \%$ ). The surface-consistent matching filters have matched the amplitude variations and the time differences between the two surveys. However, residual statics have been equalized and therefore are ideal for simultaneous surface-consistent residual statics. Stacking actually increases the NRMS for trace-by-trace matching filters while it decreases for surface-consistent matching filters.

\subsubsection{The effects of the surface-consistent terms}

Computing the four components (source, receiver, offset, and midpoint) but only applying two terms (source and receiver) is a common practice for residual statics solutions. However, because we are introducing a new surface-consistent method, we want to compare the results of applying two terms, three terms and all four terms. The results displayed in Figures 4.11a, 4.11c, and 4.11e are difference plots for two, three, and four 
components, respectively, for a single shot. The two-component difference in the prestack example seems to have resolved some of the amplitude variation, time shifts, and phase. Its mean NRMS is 30\% (Figure 4.11a), and similarly after stacking the gathers (Figure 4.11b), the residual remains relatively high with mean NRMS of about $32 \%$. Since we are computing NRMS in the window just above the reservoir, source and receiver terms will not describe all the changes in this interval, as in the surface-consistent model, and much of the changes will be described by offset and midpoint components.

Application of three components (Figure 4.11c) results in some residual amplitude and a calculated mean NRMS of about $28.5 \%$, while after stacking the gathers the difference decreased significantly, with mean NRMS of about 18.7\% (Figure 4.11d). The fourcomponent solution (Figure 4.11e) shows slight improvement over the three-component solution, and its mean NRMS is about 26.8\%. Its stacked result in Figure 4.11f has the smallest residual of all, with a mean NRMS of about $15.7 \%$.

A poststack Kirchhoff time migration was applied to the baseline survey, monitor survey, and the matched monitor survey. Figure 4.12a shows the difference between the migrated baseline stack and the migrated monitor stack with a poststack time shift of $35 \mathrm{~ms}$. Note that this time shift is not enough to reduce the amplitude residual and the variable time difference between traces in both surveys. The migrated difference between the baseline stack and the four-components surface-consistent matched monitor stack is shown in Figure 4.12b. A mean NRMS of about $13.6 \%$ is computed in the window of analysis.

In addition to seismic displays, Figure 4.13 illustrates the the average amplitude spectra of the migrated baseline stack (black line), the migrated monitor stack (gray line), and the migrated matched monitor stack (dashed line). The surface-consistent matching filters significantly reduced amplitude mismatch between the baseline and the monitor. 
Figure 4.14 illustrates the NRMS difference after different stages of processing for two different data sets. A published example of real data set from Gulf of Mexico by Helgerud et al. (2011) shows the NRMS is about $70 \%$ at the raw stack stage, and at final stack they reported an improvement to about $28 \%$. In our modeled data set we started with NRMS of about $144.7 \%$ in the prestack stage, then about $70 \%$ in the raw stack (similar to the published example), then a significant improvement after the four-component surface-consistent matching filters; and after final stack the NRMS is about $13.6 \%$. The matching filter technique used by Helgerud et al. (2011) is a post-stack process, and in contrast, we applied prestack four-component surface-consistent matching filters.

\subsection{Summary}

Using a synthetic seismic model, we applied the four-term surface-consistent matching filters to the monitor survey to match it to the baseline survey over a temporal window where changes were not expected. The prestack nonrepeatable difference between the matched monitor survey and the baseline survey was quite small compared to the difference before applying the matching filters. The simulated nonrepeatable effects are quite complex and include reflection amplitude variations, wavelet bandwidth and phase differences, and large static shifts averaging 26ms. We have demonstrated that on single trace gathers, trace-sequential matching filters could reduce the differences between time-lapse surveys when compared to surface-consistent matching filters. However, after two iterations of simultaneous surface-consistent static correction and stacking, the trace-sequential matching exhibits high NRMS (35\%) while the surface-consistent matching filters improve significantly (NRMS of 15.7\%). We have also shown that applying the four-component surface-consistent matching filters yielded the lowest NRMS difference $(15.7 \%)$ compared to applying only two-term or even three-term surface-consistent 
matching filters. Migration shows slight improvement with a mean NRMS of about $13.6 \%$. 


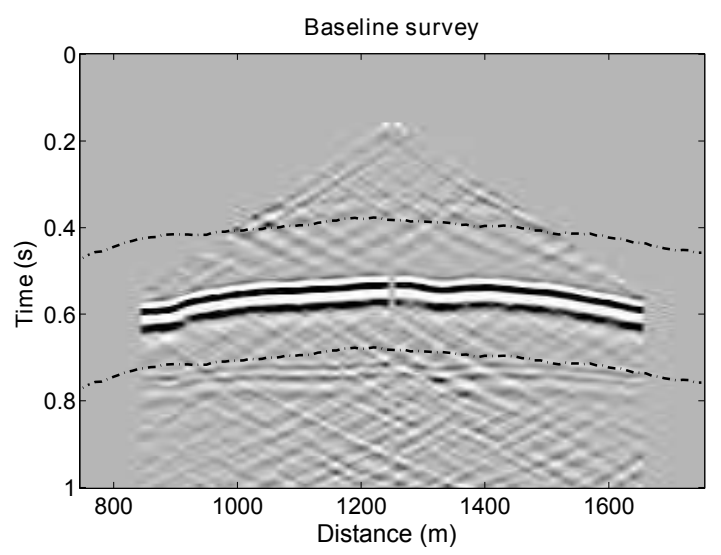

(a)

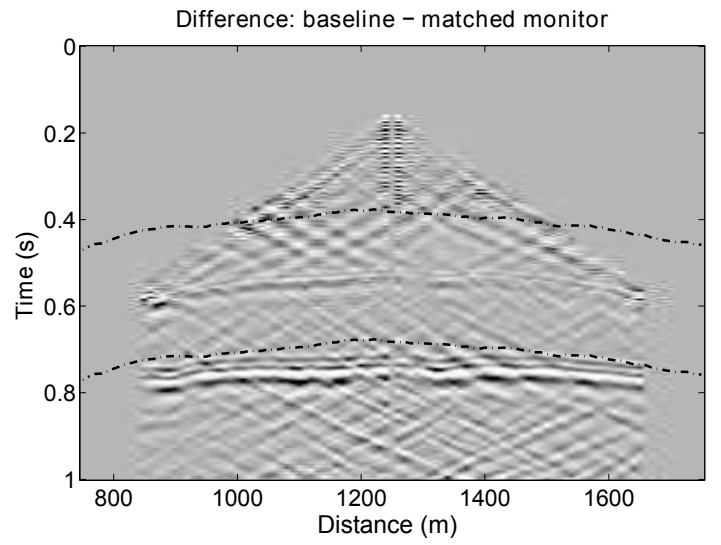

(c)

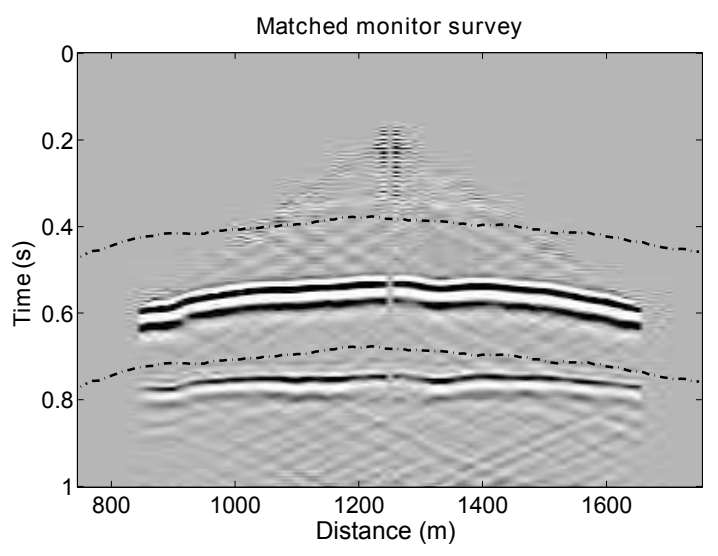

(b)

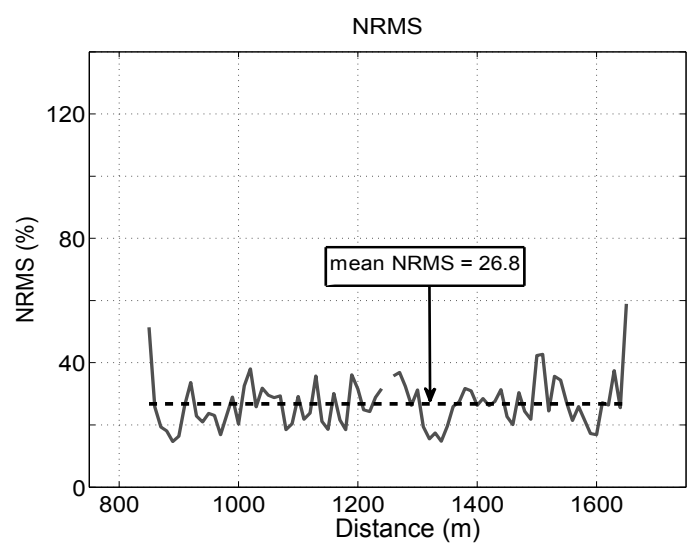

(d)

Figure 4.9: Baseline shot shown in (a), the monitor shot after applying the four-component filters to it in (b) (hereafter we named it matched monitor survey). The difference is shown in (c) and the computed NRMS between the two dashed lines is shown in (d). Note that the mean NRMS is down to $26.8 \%$. All plots have the same gain as the baseline. 


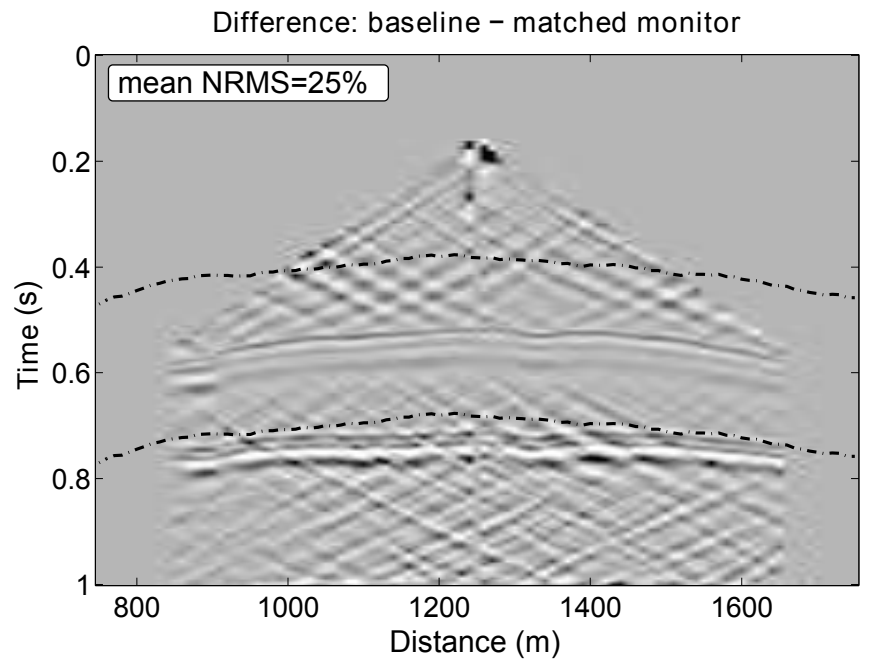

(a)

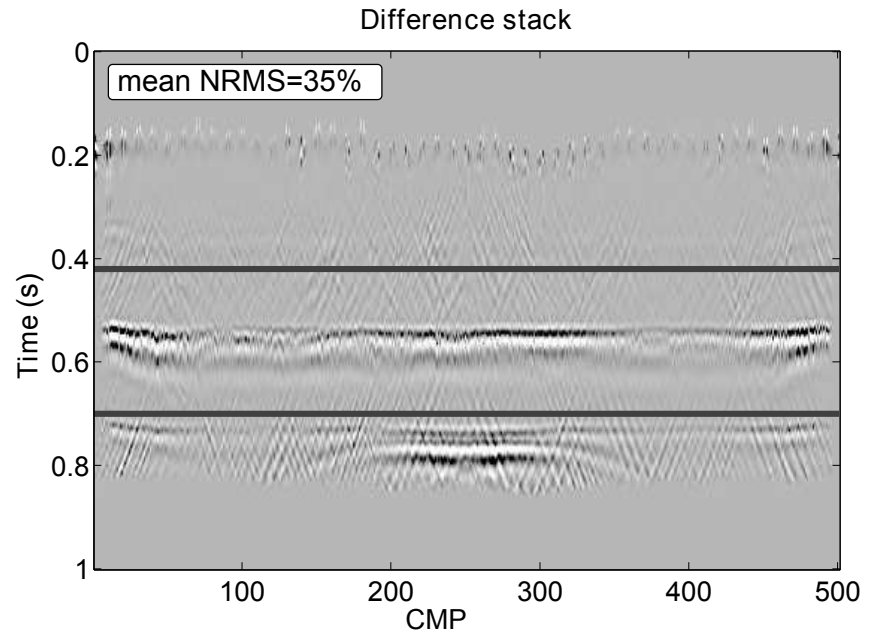

(b)

Figure 4.10: In (a) is a difference plot between baseline shot and a trace-by-trace matched monitor shot. (b) stack of the difference between baseline and a trace-by-trace matched monitor. 


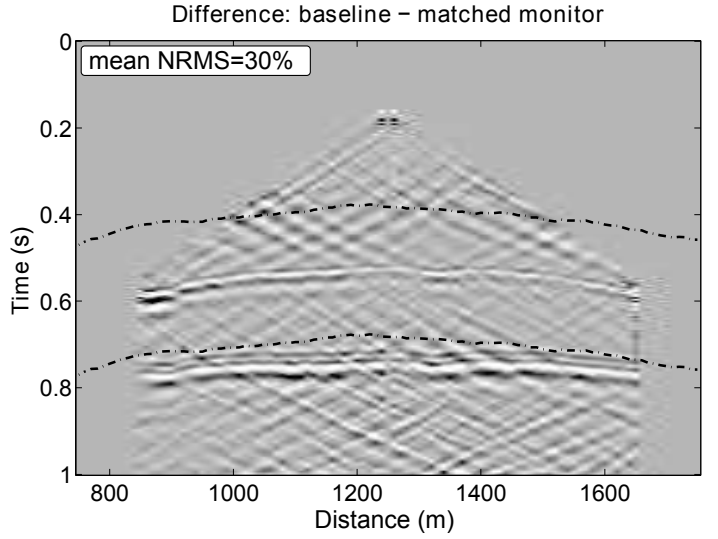

(a)

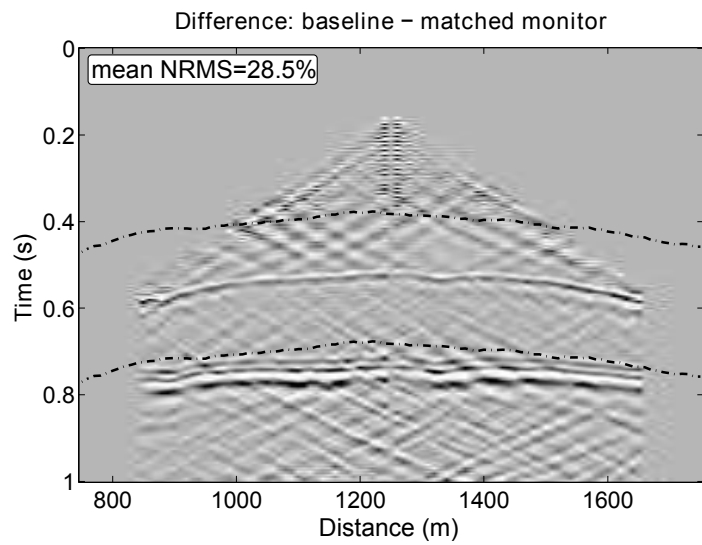

(c)

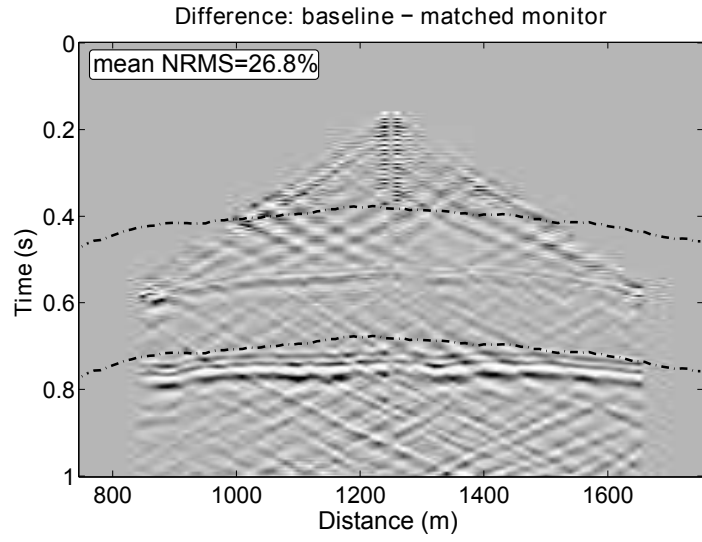

(e)

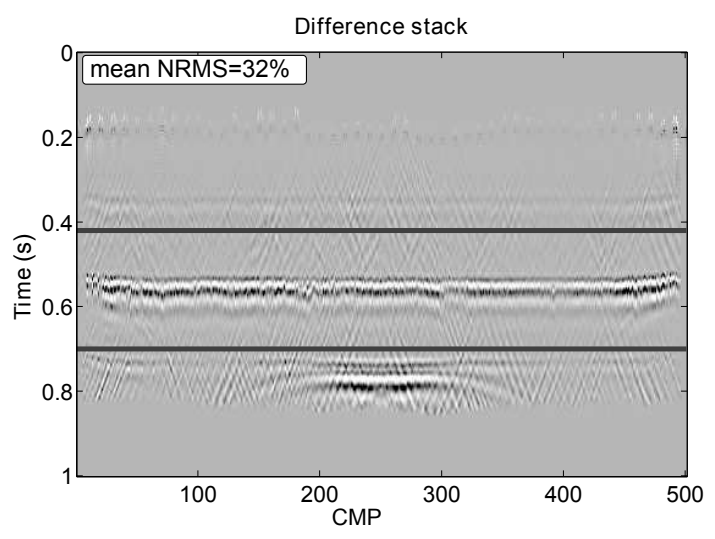

(b)

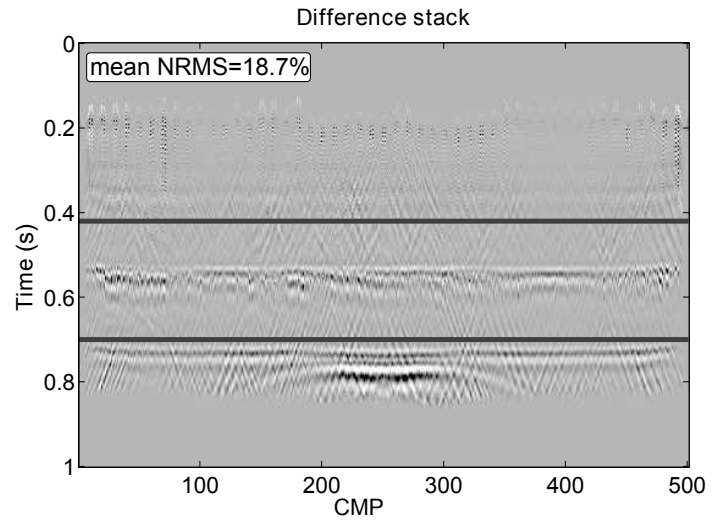

(d)

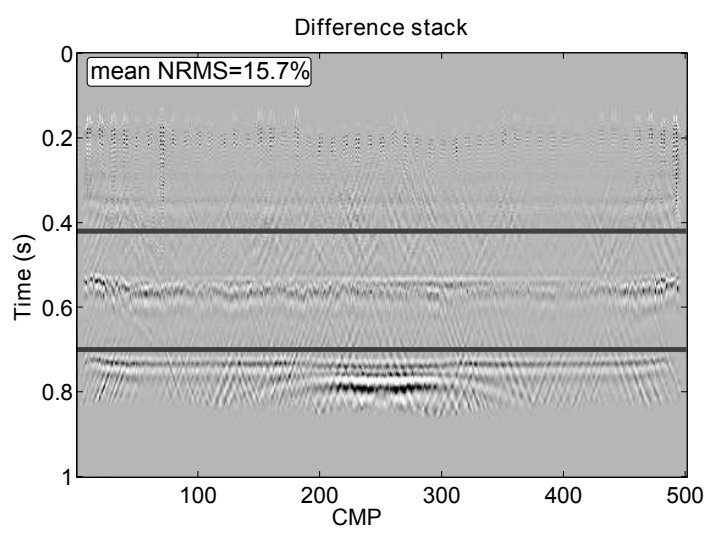

(f)

Figure 4.11: (a) is a prestack difference of the baseline and the matched monitor, with source and receiver terms applied, and (b) shows the poststack difference. (c) is a prestack difference with three terms applied (source, receiver and offset) also in (d) is the poststack difference. (e) is a prestack difference plot with four-term filters applied and (f) is the poststack difference. 


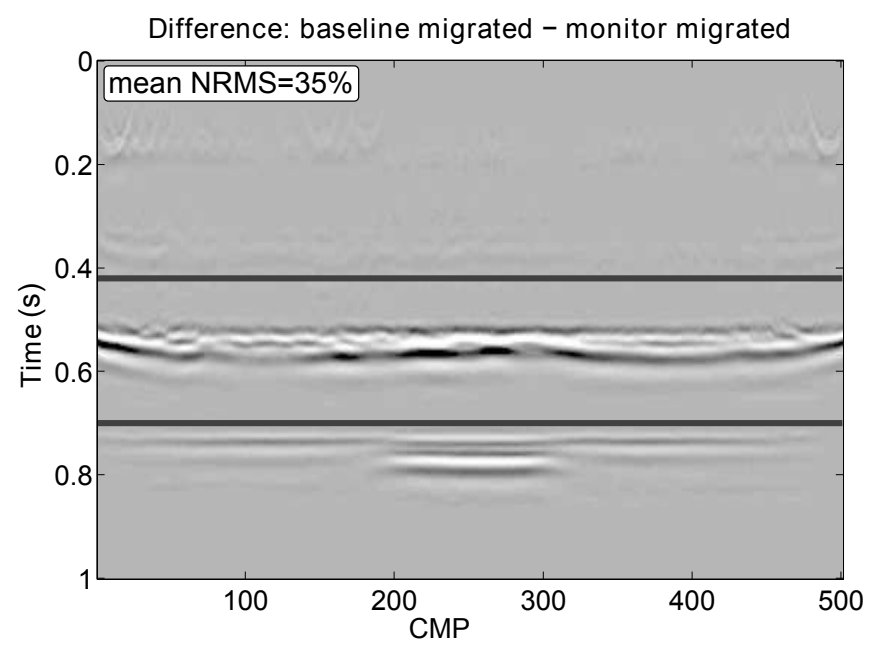

(a)

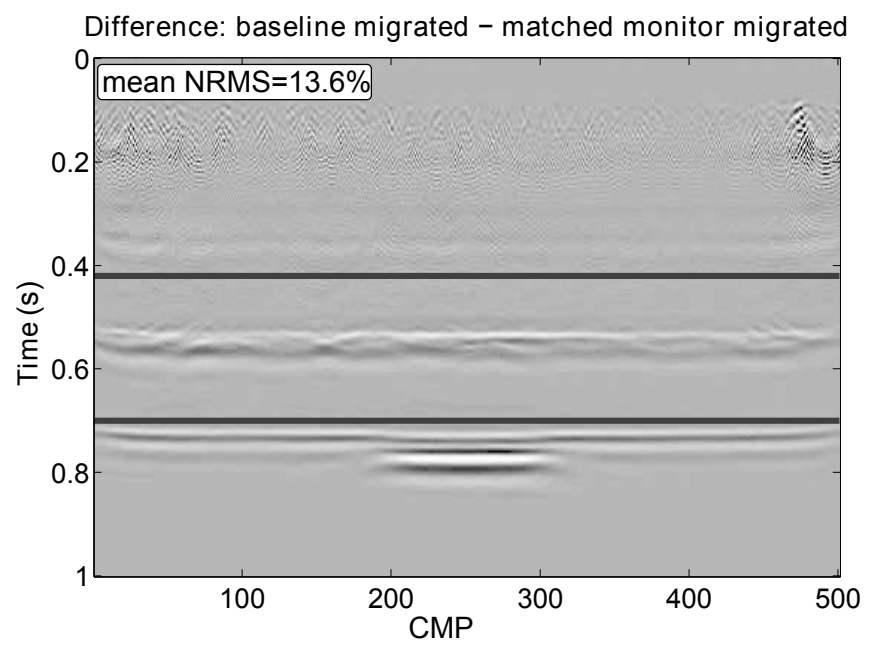

(b)

Figure 4.12: In (a) is the migrated difference plot between baseline survey and monitor survey with a poststack time shift of $35 \mathrm{~ms}$ and in (b) is the migrated difference stack between baseline and four-terms surface-consistent matched monitor. 


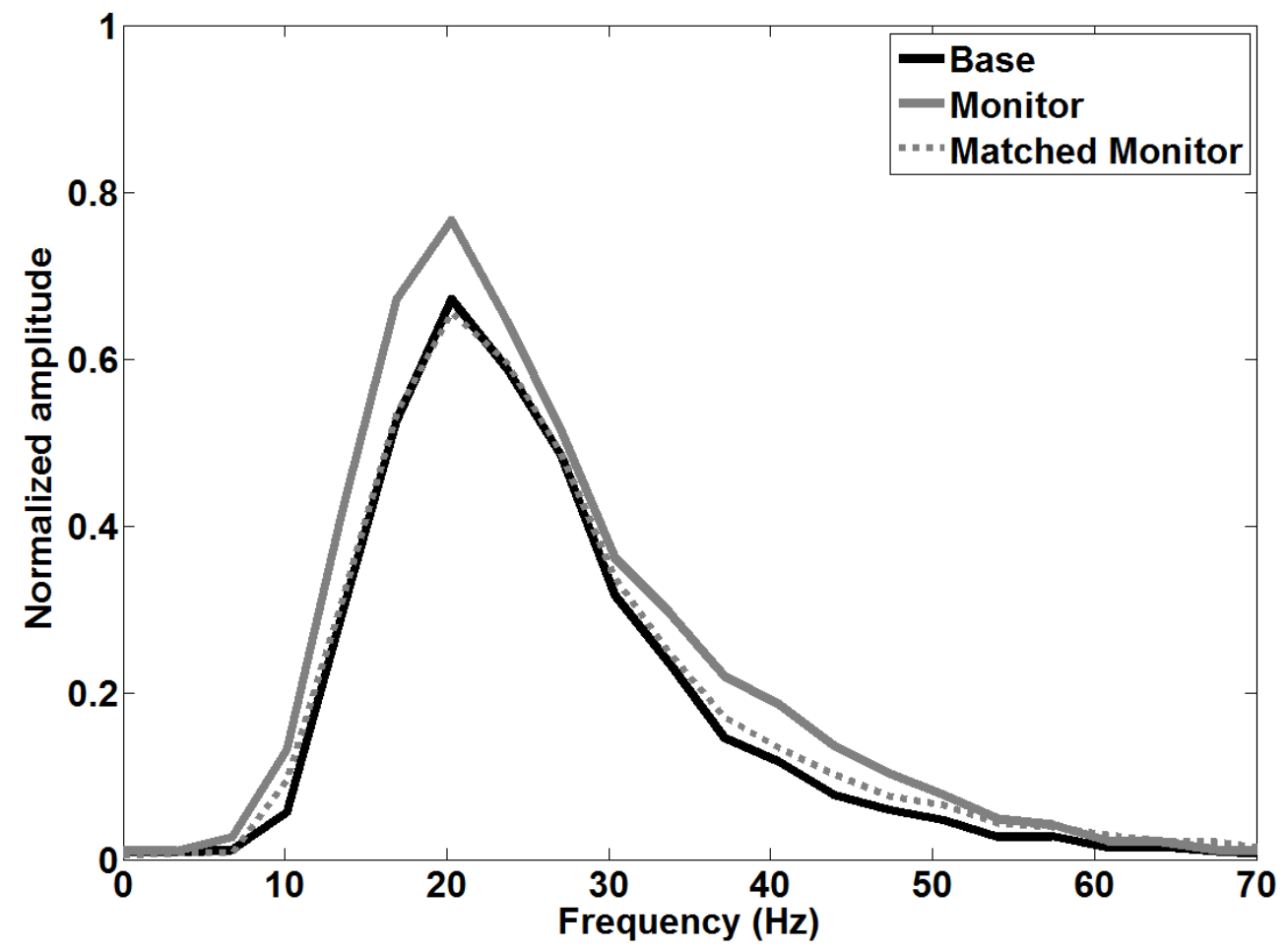

Figure 4.13: Average amplitude spectra of migrated stacks of baseline survey (black line), monitor survey (gray line), and matched monitor (dashed line) computed from all traces inside the window of analysis. Amplitude spectrum of the matched monitor survey matches nicely the baseline amplitude spectrum. 


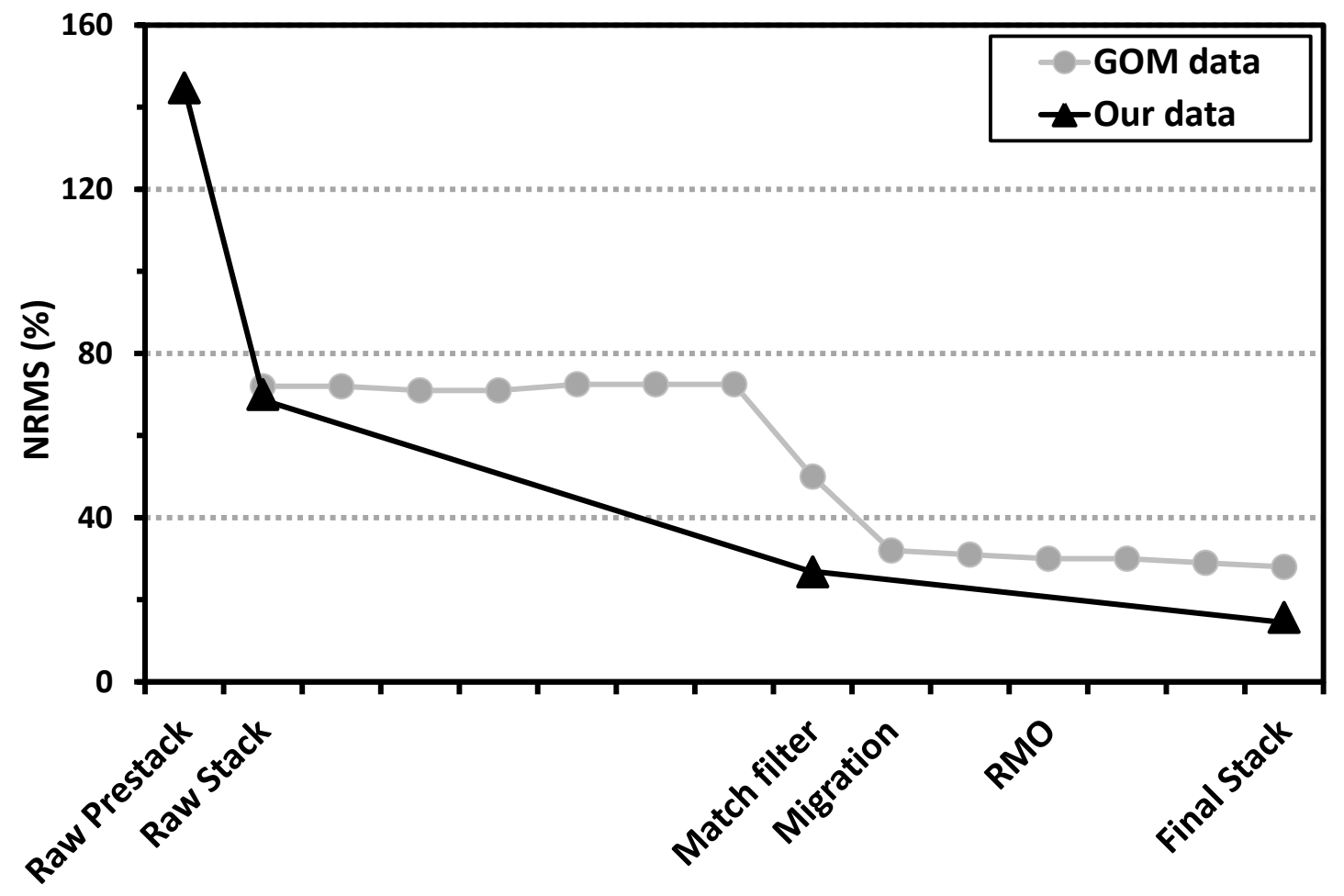

Figure 4.14: NRMS difference after different stages of processing for two different data sets. The light gray line represent a published example of real data set from Gulf of Mexico by Helgerud et al. (2011) where at raw stack stage the NRMS is about $70 \%$ and at final stack they reported an improvement of about $28 \%$. In our modeled data set we started with NRMS of about $144.7 \%$ in prestack stage, then about $70 \%$ at raw stack, then a significant improvement after the four-component surface-consistent matching filters and after final stack the NRMS is about $13.6 \%$. 


\section{Chapter 5}

\section{SCMF: application to a $\mathrm{CO}_{2}$ monitoring study}

\subsection{Introduction}

$\mathrm{CO}_{2}$ sequestration, also known as carbon capture and storage (CCS) in deep geological formations, is a multidisciplinary technology that involves capturing, transporting, and storing $\mathrm{CO}_{2}$ gas. It is a solution to a man-made problem known as global warming which is caused by increasing $\mathrm{CO}_{2}$ gas associated with fossil fuels combustion emissions into the atmosphere. The main goal of these CCS projects is to reduce the amount of greenhouse gases, and hence slow down global warming. $\mathrm{CO}_{2}$ injection is also a relatively new mechanism for EOR (Enhanced Oil recovery). CCS has been implemented in several projects such as the Sleipner field (North Sea) and the Weyburn project in Canada. This idea of storing gas deep in the ground is gaining momentum with potential new test sites around the globe.

The Sleipner field offshore Norway is one of the large scale industrial CCS projects, begun in the mid 1990's by Statoil, with the intention of storing more than 11 million tons of $\mathrm{CO}_{2}$ (Zweigel et al., 2001; Arts et al., 2002; Chadwick et al., 2009). $\mathrm{CO}_{2}$, separated from natural gas produced at the Sleipner field, is being injected into the Utsira formation at a depth of $1012 \mathrm{~m}$ below sea level and about $200 \mathrm{~m}$ below the top of the reservoir. The Utsira is highly porous (30-40\%), poorly consolidated, and highly permeable (1-3 Darcy) sandstone where brine is being replaced by $\mathrm{CO}_{2}$ (Arts et al., 2008). The Utsira formation is capped by the thick shale of the Nordland formation (200-300m).

Some of the main challenges for geoscientists are to monitor the injected $\mathrm{CO}_{2}$ in the subsurface for safety and legal concerns. The consortium involving Statoil decided 
to acquire time-lapse seismic surveys every two years with the first repeat 3D seismic in 1999, then 2001, 2002, 2004, 2006, 2008, and 2010 (Williams and Chadwick, 2012; Clochard et al., 2010). The baseline survey was acquired prior to gas sequestration in 1994. The effect of the $\mathrm{CO}_{2}$ on the seismic is evident, where the plume is imaged on the seismic data as several high amplitude sub-horizontal reflections (Figure 5.1) (Williams and Chadwick, 2012). The high negative amplitudes are due to the presence of gas saturating the sand layers, overlain by the tight mudstone. Modeling showed that the presence of gas induces a dramatic decrease of the $\mathrm{P}$-wave velocity even for moderate $\mathrm{CO}_{2}$ saturation, and consequently impacts the Top Utsira Formation reflection coefficient. $25 \% \mathrm{CO}_{2}$ saturation doubles the reflected amplitudes, while $100 \%$ gas saturation triples the reflected amplitudes (Eiken et al., 2000). Another seismic indication of the subsurface properties due to gas injection is the measured velocity pushdown determined by crosscorrelating the seismic traces between the baseline and the different monitoring surveys. Arts et al. (2008) reported a measured time-shift of more than $30 \mathrm{~ms}$ at the base of the Utsira formation which corresponds to a local 100\% gas saturation of more than $90 \mathrm{~m}$ rock column. The two main factors determining the seismic response are: 1) the large negative reflectivity contrast between the sealing mudstone and the underlying sand reservoir after being saturated with $\mathrm{CO}_{2}$, and 2) the tuning effects caused by interference from multiple zones (water-saturated sand, mudstone, $\mathrm{CO}_{2}$-saturated sand and so on) (Arts et al., 2008).

The other large industrial CCS project is the Weyburn Field from onshore Canada, with an estimated 1.9 billion barrel of oil in place $\left(\sim 29^{\circ} \mathrm{API}\right)$, located on the northeast flank of the Williston Basin in southeast Saskatchewan (Li, 2003). The Weyburn-Midale carbonate reservoir consists of two distinct zones at a depth of about $1450 \mathrm{~m}$. The upper "Marly" dolostone zone is a high porosity ( 26\%), low permeability $(10 \mathrm{mD})$, and 7 to $10 \mathrm{~m}$ thickness. The lower "Vuggy" limestone-dominated zone has low porosity ( 

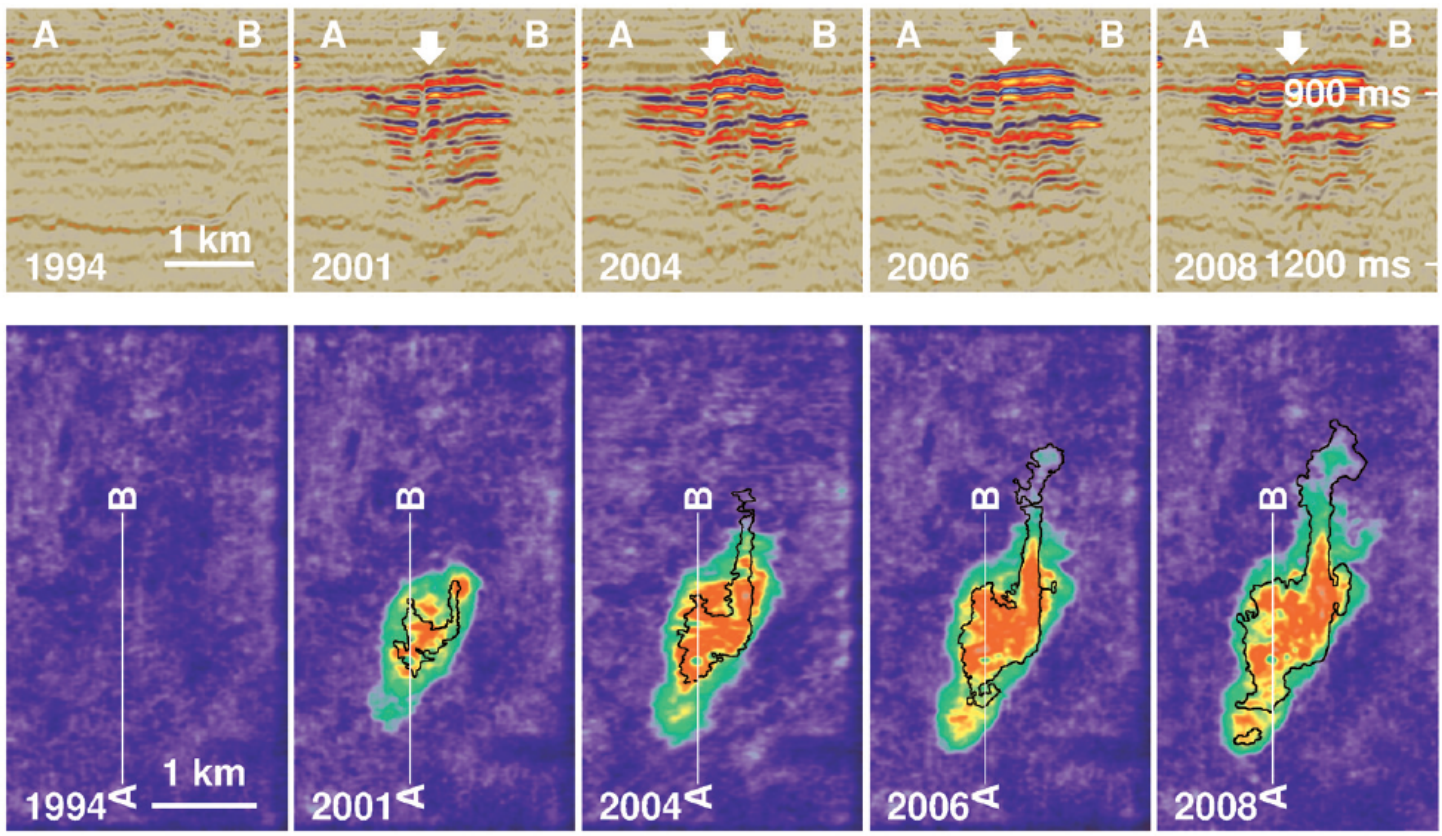

Figure 5.1: Top panels show a cross section from the time-lapse 3D seismic surveys at the Sleipner field. Note the strong $\mathrm{CO}_{2}$ plume signature on the 2001 survey onwards. Bottom panels show map view of the total reflection amplitude of the plume (Williams and Chadwick, 2012). 
$11 \%$ ), high permeability $(15 \mathrm{mD})$, and 15 to $20 \mathrm{~m}$ thickness. Both zones are known to be dominated by subvertical natural fractures, both open and cemented. The field achieved about $25 \%$ of oil recovery since the start of waterflooding 10 years after the field's discovery in 1955 ( Li, 2003).

Encana, the field operator, started the $\mathrm{CO}_{2}$ miscible flood with 19 horizontal wells in October 2000 in an attempt to recover additional 15\% of the original oil in place (Davis et al., 2003; Li, 2003). The process of EOR is expected to take about 25 years by injecting an estimated 14 million tons of $\mathrm{CO}_{2}$, with the focus on the unswept oil in the porous Marly dolostones (Li, 2003). In an effort to monitor the flooding, multicomponent timelapse seismic surveys (including surface seismic, 4D VSP, and crosswell) are acquired. The first baseline survey was acquired in December 1999, prior to gas injection, followed by monitor surveys in 2001, 2002, 2004, and 2007 with similar acquisition parameters (Li, 2003; White, 2009). Some of the key factors to the success of the Weyburn timelapse data include repeatability in acquisition and processing, vertical resolution of the thin zones, and detectability of dynamic fluid changes (Li, 2003). Processing the timelapse data was a critical part of the project especially when the seismic response of the $\mathrm{CO}_{2}$ injection is estimated to be small compared to the interference of the nonrepeatable noise. Figure 5.2 is a NW-SE oriented migrated P-wave section from the baseline and the monitor surveys. The seismic section crosses four SSWG (simultaneous but separate water and gas injection) wells. The two thin reservoirs, Marly and Vuggy, have been well resolved by a strong trough and a relatively weak peak, respectively ( $\mathrm{Li}, 2003$ ). Rock and fluid physics modeling and measurements for the Weyburn Field suggested that $\mathrm{CO}_{2}$ flooding would cause a decrease of $4-10 \%$ in P-wave velocity with corresponding 15-20\% change in seismic impedance, even at low levels of saturation (5-10\%), whereas pressure effects are negligible (Davis et al., 2003; White, 2009). In addition to amplitude increase from baseline to monitor, there has been a measurable time-delay introduced by the $\mathrm{CO}_{2}$ 
flooding where as much as $4 \mathrm{~ms}$ difference was detected near the reservoir zones and up to 2 ms time-delays for the deeper Bakken formation (Figure 5.3) (White, 2009). In most cases, there is an agreement between the seismic response changes and the volume of gas injected, particularly in the southern part of the field where the Marly unit is more porous and all injector wells are horizonal. In the northern part of the field, the reservoir is tight and the injector wells are vertical, and even though large volumes of $\mathrm{CO}_{2}$ have been injected, the seismic anomalies are not as strong (White, 2009).

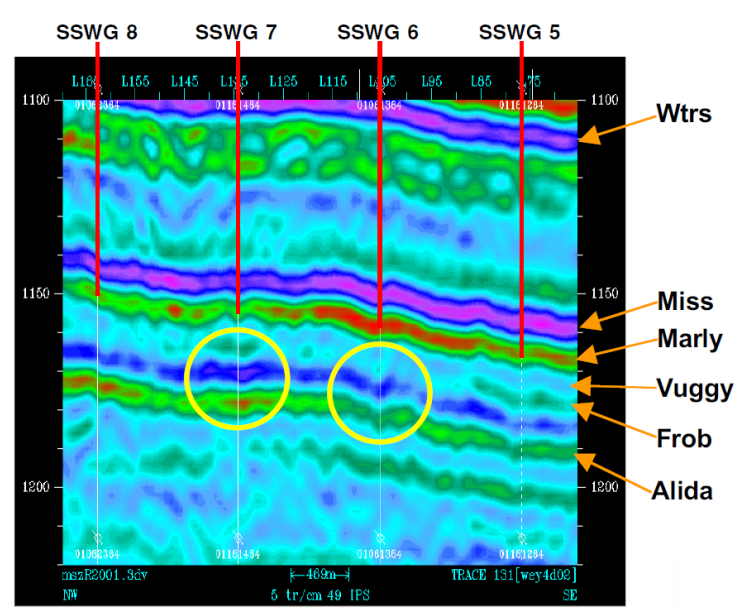

(a)

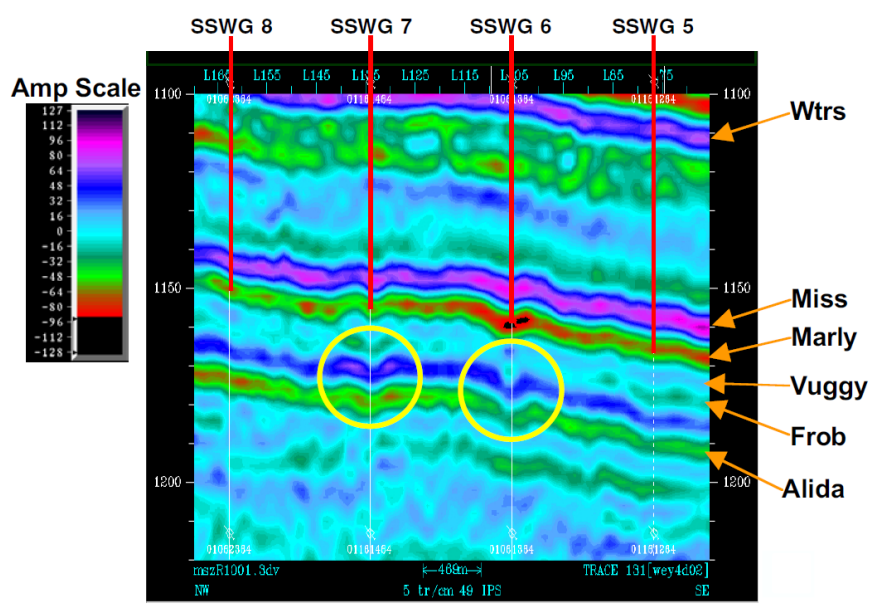

(b)

Figure 5.2: A NW-SE oriented migrated P-wave section from the baseline (a) and the 2001 monitor (b) showing well resolved Marly and Vuggy zones and increasing amplitude signature due to the injected gas (Li, 2003).

The presented examples demonstrate the value of the seismic monitoring to CCS projects. The value of the seismic depends on many factors including: acoustic properties of reservoir fluid, porosity, lithology, reservoir pressure, reservoir temperature changes, and geomechanical changes. Therefore, to obtain a successful seismic monitoring program, it is essential to begin with rock and fluid physics modeling in order to understand the factors that might have greater impact on the seismic monitoring response. The response of the seismic in the Sleipner study is different from that of the Weyburn field, largely due to lithology differences between the two fields, highly porous and highly un- 


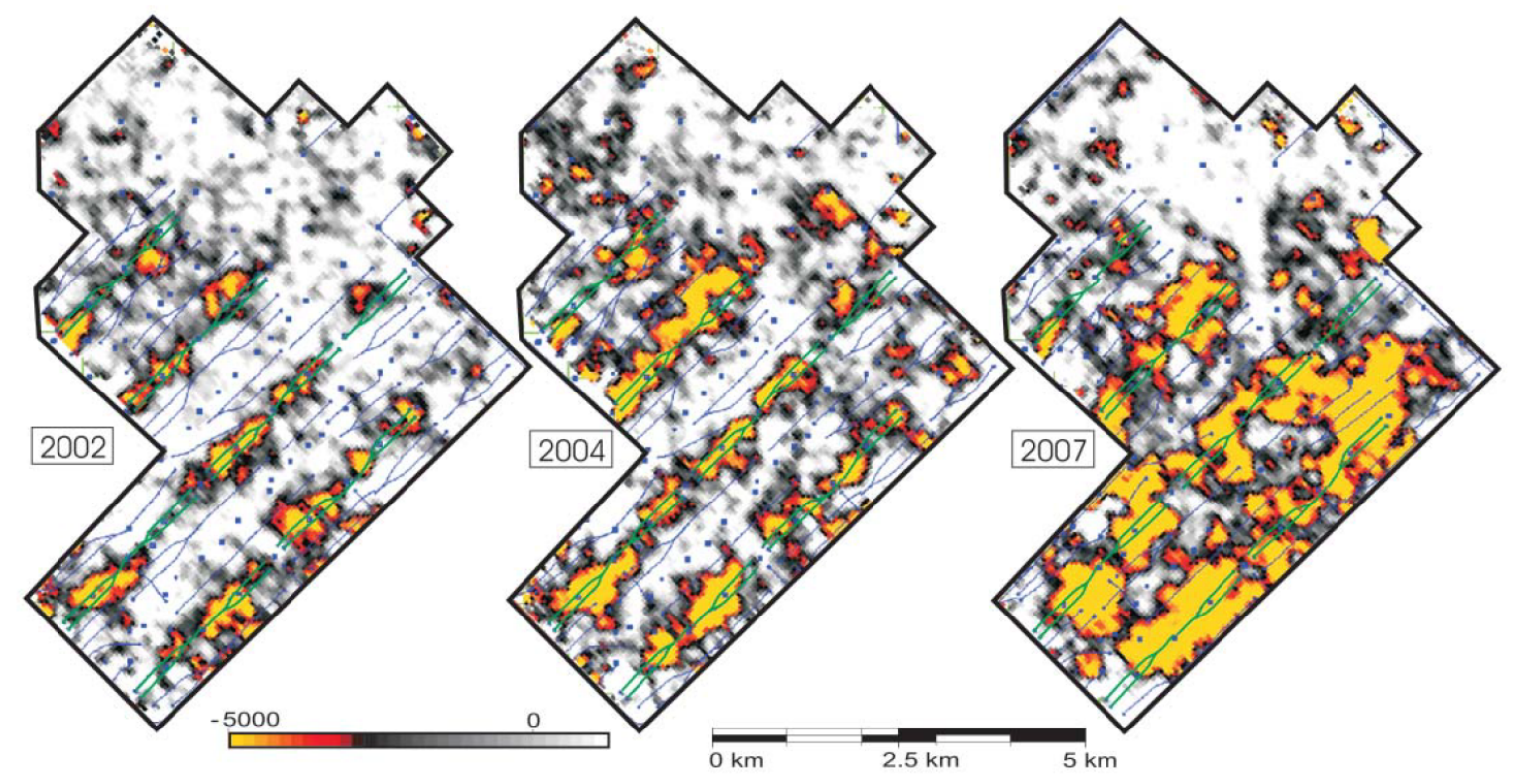

(a)

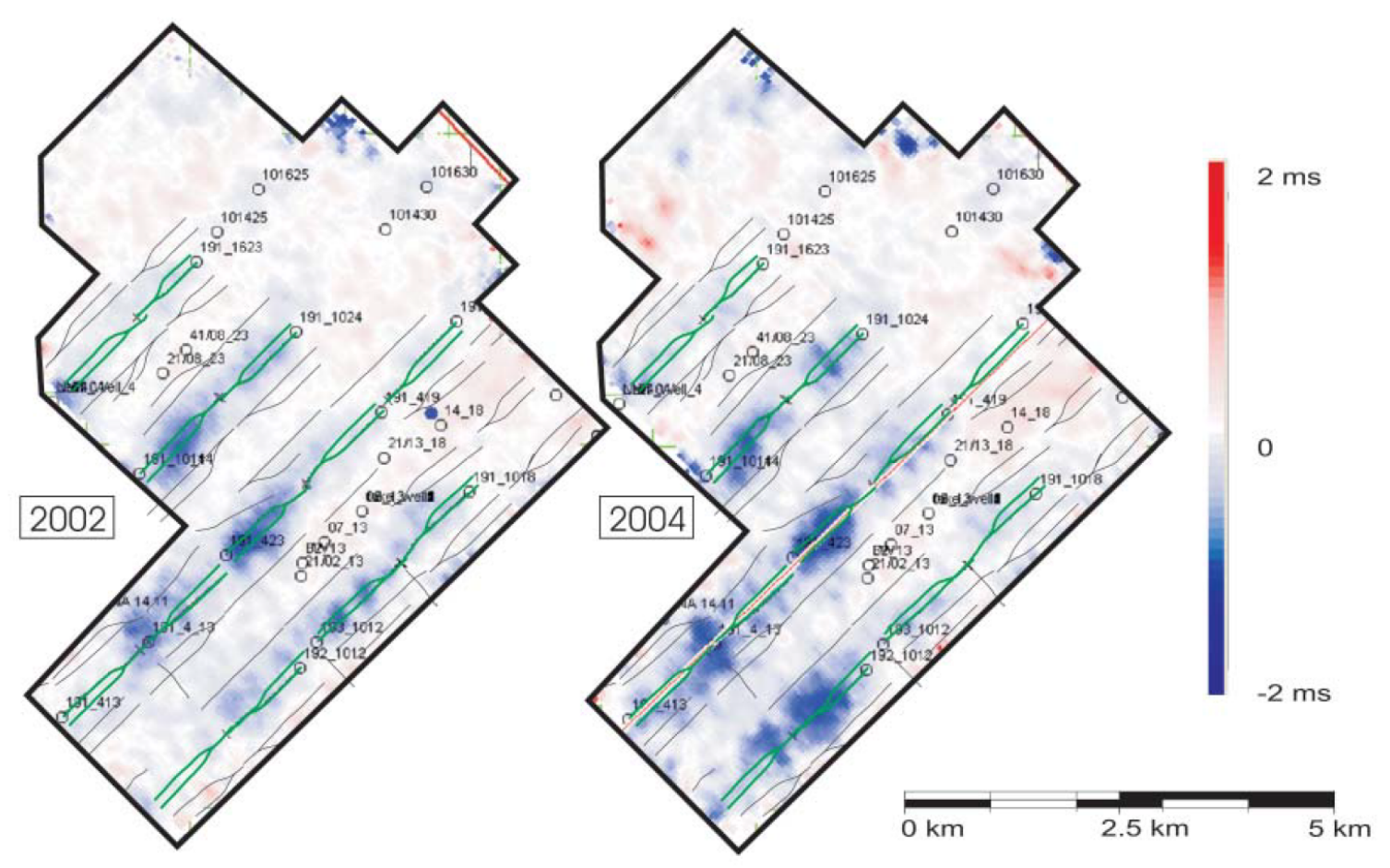

(b)

Figure 5.3: (a) shows a time-lapse amplitude difference map extracted from the upper reservoir unit and (b) shows time-delays present in time-lapse difference map for the deep Bakken formation (White, 2009). 
consolidated clastic sandstone in the Sleipner field versus a moderately porous carbonate rock in the Weyburn field.

The quality of the seismic monitoring response depends on the quality of acquisition, the quality of processing, and the strength of the time-lapse signal. High quality processing will reduce the nonrepeatable noise, compensate for nonrepeatability in acquisition, enhance the seismic repeatability, and improve the subsurface time-lapse differences due to injected fluids.

In this chapter, we present an investigation of the SCMF algorithm applied to $\mathrm{CO}_{2}$ monitoring seismic data from the Pembina field, known as the Violet Grove $\mathrm{CO}_{2}$ pilot study, located in central Alberta, Canada. The analysis will include a review of the testing site, a review of the geology and geophysics data, and the results of a time-lapse 2D line processed by two different methods: 1) parallel processing, and 2) simultaneous processing with SCMF. Both processing methods have been described in Chapter 4.

\subsection{Violet Grove $\mathrm{CO}_{2}$ pilot study}

The Violet Grove site, part of the Pembina oil field near Drayton Vally, $100 \mathrm{~km}$ southwest of Edmonton, in central Alberta (Figure 5.4), was chosen by PennWest Petroleum Ltd., the operator, for a $\mathrm{CO}_{2}$-EOR and CCS pilot study. In collaboration with the Alberta Energy Research Institute (AERI) and the Western Economic Diversification (WED), a $\mathrm{CO}_{2}$ monitoring program, that involved 2D and sparse 3D multi-component surface seismic as well as borehole seismic data, was acquired, processed and analyzed with the baseline data acquired in March 2005 and the last monitoring acquired in March 2007 (Lawton et al., 2005; Coueslan, 2007; Alshuhail, 2011). 


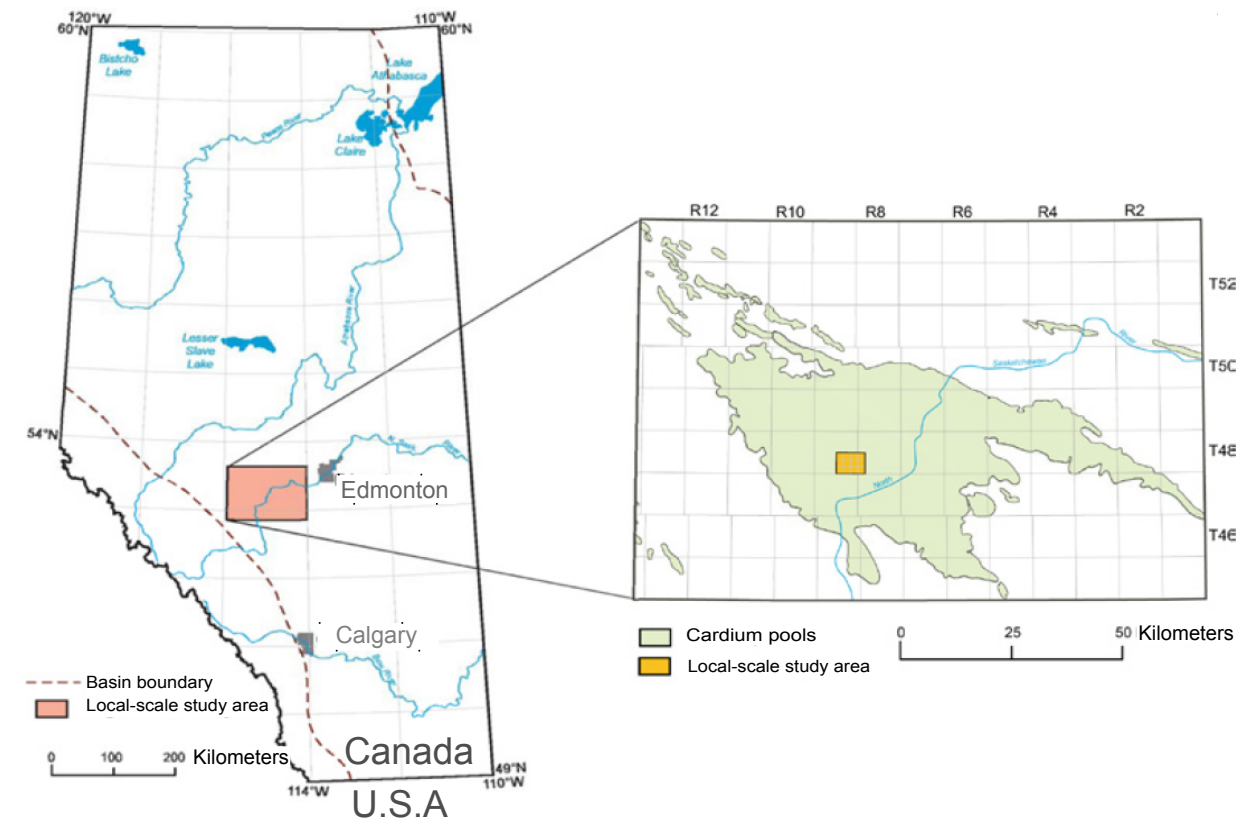

Figure 5.4: Map of Alberta showing the location of the Pembina Oil Field and the $\mathrm{CO}_{2}$-EOR pilot site at Violet Grove (Dashtgard et al., 2006).

\subsubsection{Geology of the area}

The Pembina Oil Field is the most aerially extensive oil field in the world covering approximately $4000 \mathrm{~km}^{2}$ (Dashtgard et al., 2006) with huge oil and gas reserves. Discovered in 1953, production in this field is from Devonian to Tertiary strata with the Upper Cretaceous Cardium Formation being the most prolific (Dashtgard et al., 2006).

Injection of the $\mathrm{CO}_{2}$ was in the Upper Cretaceous Cardium Formation in the period between March 2005 and March 2007 (Alshuhail et al., 2008). According to Hitchon (2009), this formation has been subdivided into the lower dominantly arenaceous Pembina River Member and the upper dominantly argilaceous Cardium Zone Member. The Pembina River Member is a coarsening-upward sequence, dominated by shale layers at the base and sandstone layers at the top, and is capped by chert conglomerate. The Cardium Formation overlies the Blackstone Formation which is dominated by black shale, and is overlain by shales of the Wapiabi Formation (Figure 5.5). At the site, the Cardium Formation consists of three distinct sandstone units (upper, middle and lower) separated by 


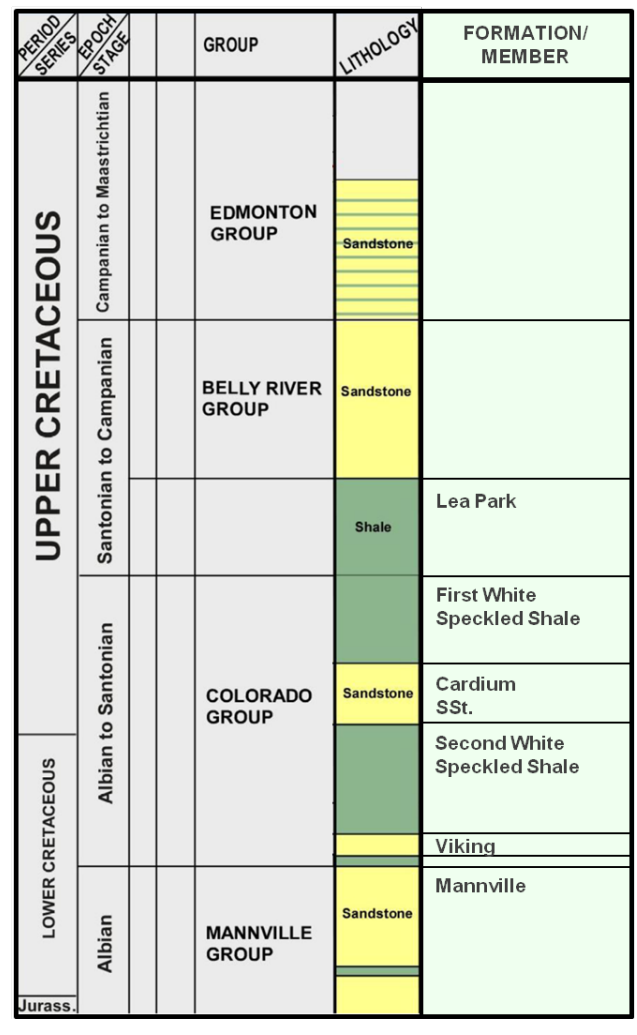

(a)

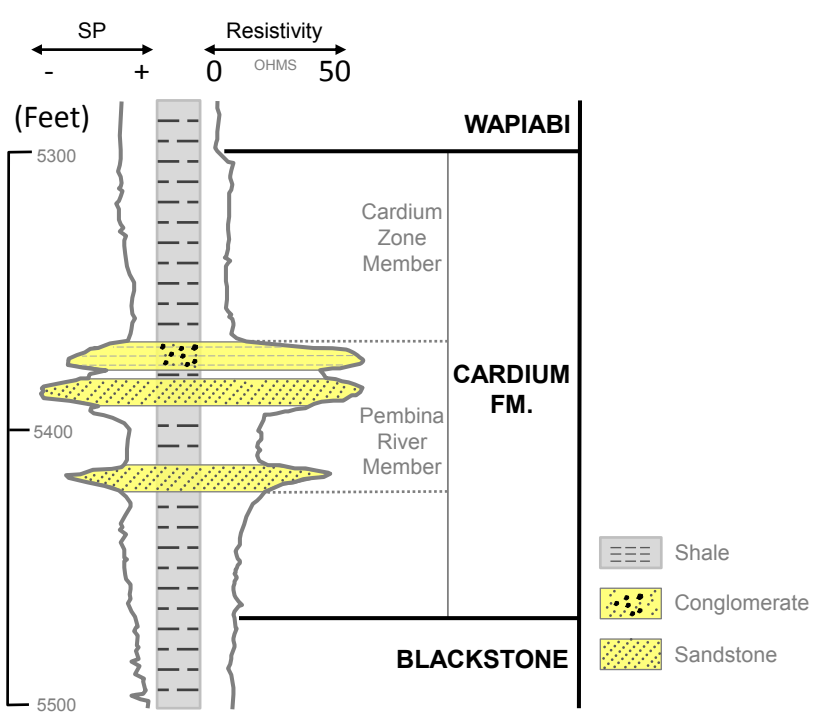

(b)

Figure 5.5: General stratigraphic column of central Alberta of the Lower and Upper Cretaceous (Bachu and Bennion, 2008) shown in (a) and a typical well log from the Pembina Field showing the Cardium Formation in (b); a modified plot of that in Patterson (1957). 
two shale units. The effective reservoir unit thickness is about $10 \mathrm{~m}$ (formation thickness is $\sim 43 \mathrm{~m}$ ) at a depth of about $1600 \mathrm{~m}$ in the northeast to about $1650 \mathrm{~m}$ in the southwest of the pilot study area. Table 5.1 summarized some of the physical properties of the Cardium Formation with average porosity, average permeability $(\mathrm{K})$ in millidarcy $(\mathrm{mD})$, temperature in degrees Celsius $\left(C^{o}\right)$, and pressure in megapascal $(\mathrm{MPa})$.

Table 5.1: Physical properties of the Cardium Formation from the study area (Hitchon, 2009).

\begin{tabular}{lcccc}
\hline Lithology & Ave. porosity $(\%)$ & Ave. K $(\mathrm{mD})$ & Temp. $\left(C^{o}\right)$ & Pressure (MPa) \\
\hline Conglomerate & 8 & 31 & 50 & 19 \\
Upper and middle sand & 16 & 21 & 50 & 19 \\
Lower sand & - & 10.5 & 50 & 19 \\
\hline
\end{tabular}

Rock-physics-based modeling to determine the acoustic response of the Cardium reservoir for different $\mathrm{CO}_{2}$ saturation and plume thicknesses was performed by Alshuhail et al. (2008). The modeling assumed a relatively tight sandstone $(\sim 10 \%)$ and a cemented matrix. $\mathrm{CO}_{2}$ injection causes a decrease in $\mathrm{P}$-wave velocity by about $4 \%$ and a density decrease by about $\sim 1 \%$, based on modified Gassman's modeling and substituting a mixed brine and light oil with supercritical, $80 \%$ saturated, $\mathrm{CO}_{2}$. The generated zerooffset P-wave traces are shown in Figure 5.6. The traces from left to right in each panel show the result of increasing $\mathrm{CO}_{2}$ plume thickness from 0 to $24 \mathrm{~m}(<10 \mathrm{~m}$ is a more likely scenario for plume thickness). The fluid replacement parameters used by Alshuhail et al. (2008) for the baseline were $50 \% \mathrm{H}_{2} \mathrm{O}$ and $50 \%$ Oil. For the monitor, they used $80 \% \mathrm{CO}_{2}, 10 \% \mathrm{H}_{2} \mathrm{O}$, and $10 \%$ Oil. The result of the modeling was a subtle reflectivity change at the Cardium and a very small time-delay at the Viking $(<0.25 \mathrm{~ms})$. 


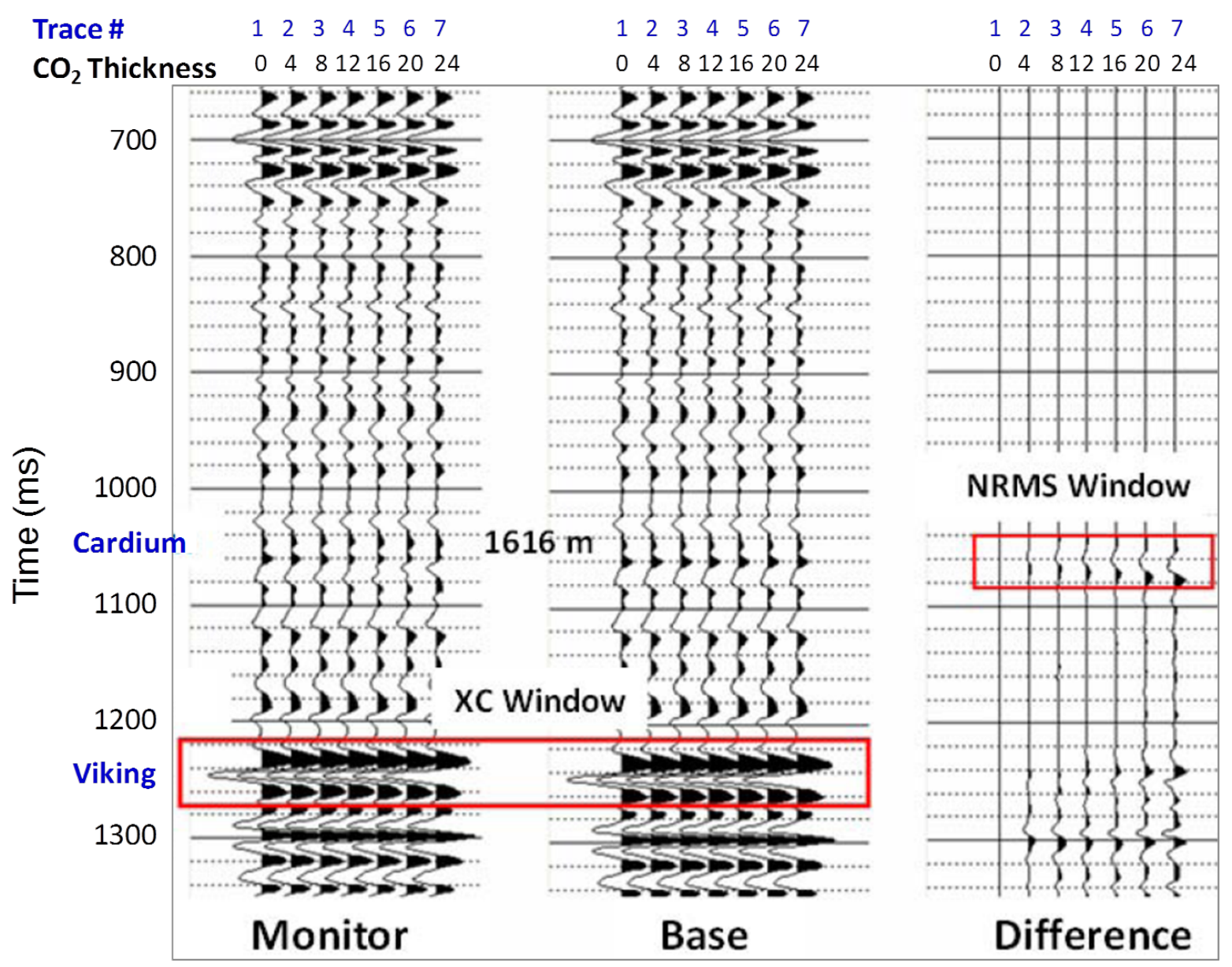

Figure 5.6: Zero-offset seismic modeling of the effects of $\mathrm{CO}_{2}$ saturation in the Cardium reservoir. (Alshuhail et al., 2008). 


\subsubsection{Geophysics data: acquisition and previous processing}

Three phases of surface and borehole seismic data were acquired at the Pembina Cardium $\mathrm{CO}_{2}$-EOR pilot site between March 2005 and March 2007. During this period, approximately 60, 000 tonnes of $\mathrm{CO}_{2}$ were injected into the Cardium Reservoir. A time-lapse seismic data set was designed and acquired as part of the monitoring program. It consisted of acquiring, processing, and interpreting 2D and 3D surface seismic and 2D vertical seismic profile (2D VSP) data sets (Figure 5.7) (Lawton et al., 2005; Alshuhail, 2011). The main objective of the seismic program is to verify the $\mathrm{CO}_{2}$ plume, and to evaluate the integrity of the storage. A baseline program (or phase I) of 2D surface seismic,

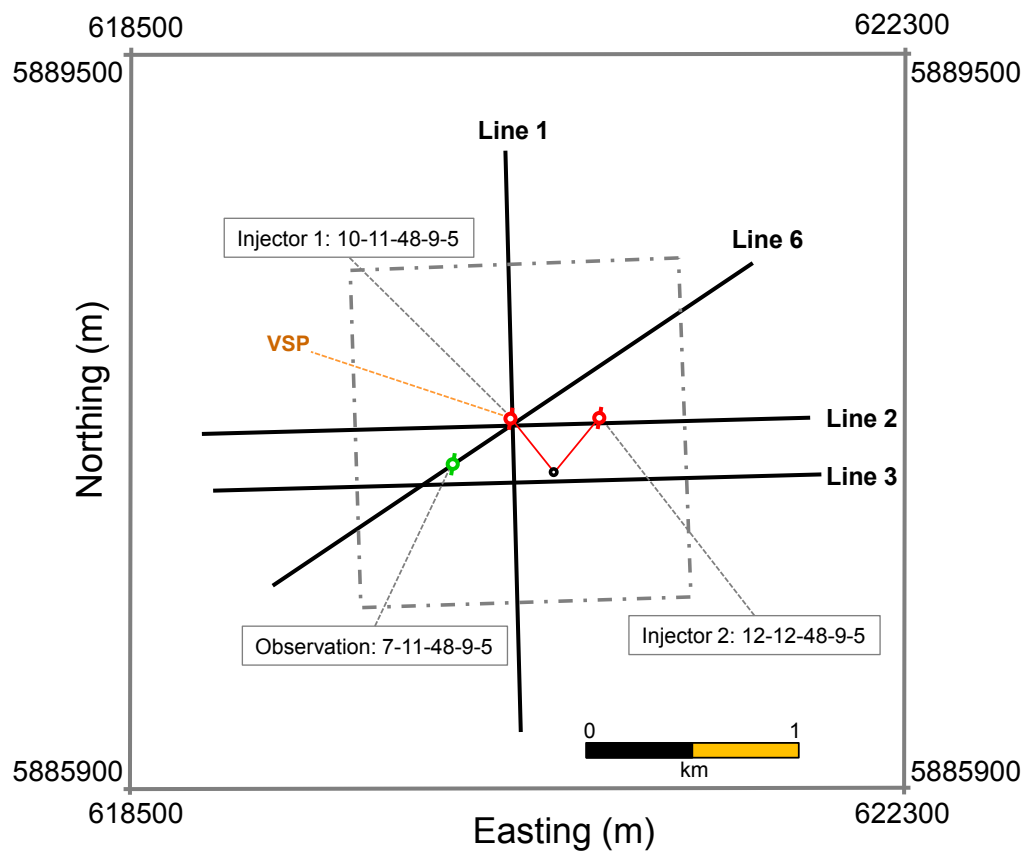

Figure 5.7: Layout of the surface seismic lines, 3D seismic coverage (indicated by the dashed rectangle), and location of the injector wells, observation well, and VSP (Lawton et al., 2005).

acquired in March 2005, consisted of two, multi-component, parallel lines, $400 \mathrm{~m}$ apart and oriented east-west. A third orthogonal line is oriented north-south intersecting the other two lines near the $\mathrm{CO}_{2}$ injector well. Each line is $3 \mathrm{~km}$ long with $20 \mathrm{~m}$ receiver 
interval, $40 \mathrm{~m}$ source spacing, $2 \mathrm{~kg}$ dynamite charges at $15 \mathrm{~m}$ depth, approximately 3000 m maximum source-receiver offset, and a record length of 3 seconds with a sample rate of $1 \mathrm{~ms}$. In addition, the observation well had eight triaxial geophones cemented at 20 m intervals at depths between $1470 \mathrm{~m}$ and $1640 \mathrm{~m}$ with the deepest geophone within the Cardium reservoir.

Phase II of the seismic data set was acquired in December 2005, with similar geometry as phase I, and after injecting approximately 15, 000 tonnes of $\mathrm{CO}_{2}$ in the reservoir. Phase III seismic program was acquired in March 2007 with similar geometry to the previous two phases, but with a record length of 4 seconds, addition of a $3 \mathrm{~km}$ long 2D line (Line 6) trending southwest-northeast, and a high resolution 16-level VSP (recorded in injector well \#1). Table 5.2 summarizes the time-lapse seismic program at the $\mathrm{CO}_{2}$-EOR study site.

Table 5.2: Summary of the seismic program at the $\mathrm{CO}_{2}$-EOR study site (Alshuhail et al., 2008).

\begin{tabular}{|c|c|c|c|}
\hline Phase & Seismic Data & Date & Injected $\mathrm{CO}_{2}$ (tonnes) \\
\hline \multirow{3}{*}{ I (baseline) } & i. Line 1 (north-south) & & \\
\hline & ii. Lines 2 and 3 (east-west) & & \\
\hline & iii. Fixed-array VSP (8 geophones) & March 2005 & 0 \\
\hline \multirow[t]{3}{*}{ II $\left(1^{s t}\right.$ monitor $)$} & i. Line 1 (north-south) & & \\
\hline & ii. Lines 2 and 3 (east-west) & & \\
\hline & iii. Fixed-array VSP (8 geophones) & December 2005 & 15,000 \\
\hline \multirow[t]{5}{*}{ III ( $2^{\text {nd }}$ monitor $)$} & i. Line 1 (north-south) & & \\
\hline & ii. Lines 2 and 3 (east-west) & & \\
\hline & iii. Fixed-array VSP (8 geophones) & & \\
\hline & $\begin{array}{l}\text { vi. Addition of - } \\
\text { (a) 16-level VSP }\end{array}$ & & \\
\hline & (b) southwest-northeast 2D line (line 6 ). & March 2007 & 60,000 \\
\hline
\end{tabular}

The acquired seismic data sets were processed independently with similar processing parameters by two processing companies ( CGGVeritas and Divestco) and internally within CREWES. Table 5.3 summarizes the processing flow of the P-wave data and has been highlighted in several CREWES reports and theses such as Lawton et al. (2005); 
Alshuhail (2011). The baseline and the monitor surveys were processed independently, but with the same parameters and the same contractor.

Table 5.3: CGGVeritas processing work flow of the 2D P-wave surface seismic data set (Lawton et al., 2005; Alshuhail, 2011).

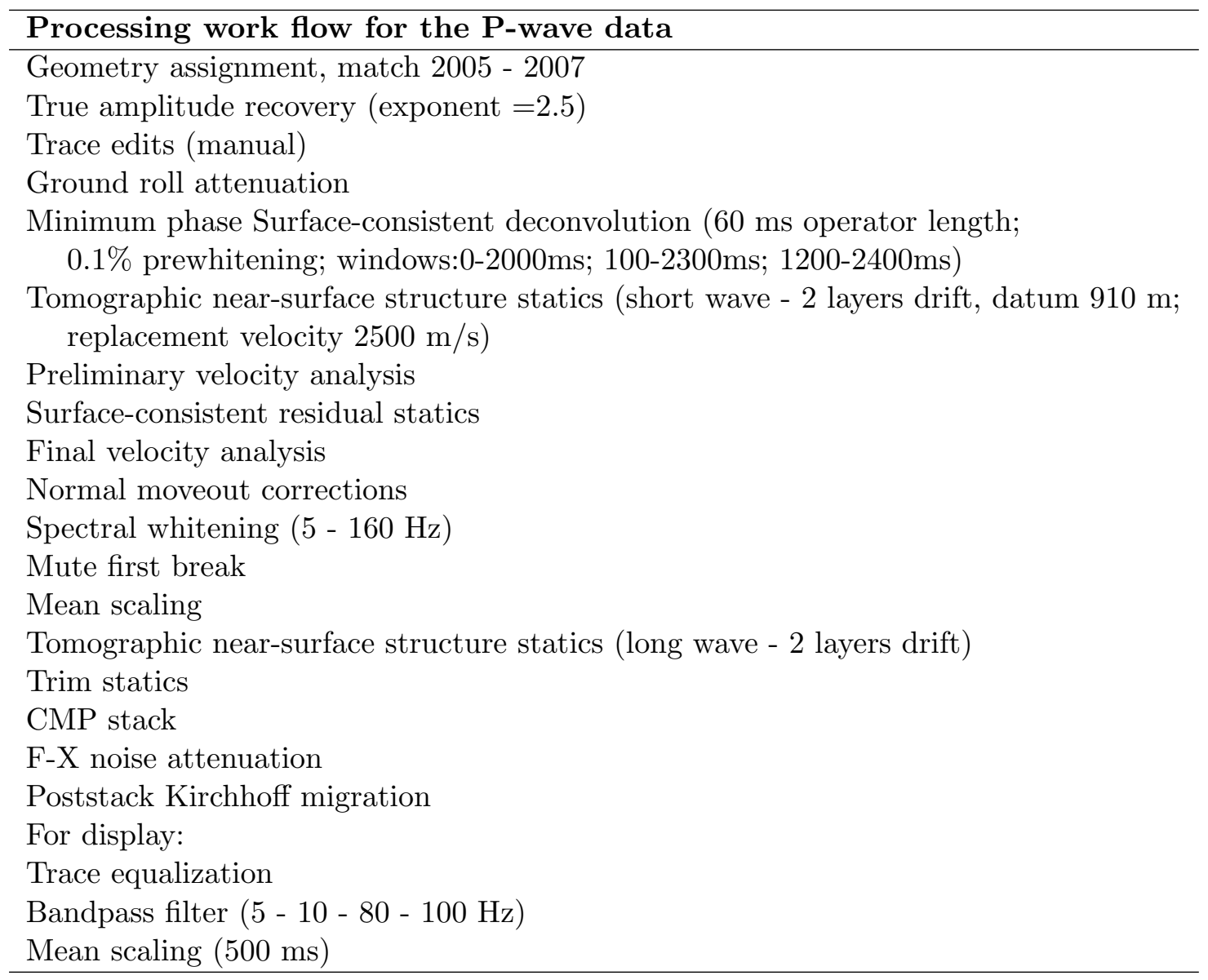

Well 102/07-11-048-09 penetrated the Cardium and the Blackstone Formations. The computed synthetic seismograms (from the acquired dipole sonic log and calculated density) matched the surface seismic data quite well (Figure 5.8). The top of the Ardley Coal Zone is a strong peak picked at a depth of $440 \mathrm{~m}$ at the well location which corresponds to $360 \mathrm{~ms}$ on the $\mathrm{P}$-wave data. The next event is the top of the Cardium Formation which correlates to a weak peak at approximately $1043 \mathrm{~ms}$ on the P-wave data (about $1610 \mathrm{~m}$ TVD) and the top of the Blackstone Formation correlates to a weak trough at 
about $1060 \mathrm{~ms}$ (about $1635 \mathrm{~m}$ TVD).

Figure 5.9 shows the processed P-wave data of Line 1 from Phase I and III only since these surveys were recorded at the begining and at the end of the monitoring project and are expected to have the largest time-lapse differences. The data quality is fair and the main reflections were easy to pick starting from the shallow top Ardley Coal Zone to the Viking Formation which is a sandstone-dominated zone. The Cardium Formation is a low impedance unit on the baseline seismic, and even after injecting the $\mathrm{CO}_{2}$ gas, it shows as a low amplitude on the monitor survey. The monitor stack has been matched to the baseline stack over a time window above the reservoir. The difference section (Figure 5.9) shows no clear anomalies at or below the Cardium Formation (Alshuhail et al., 2008). At the top of the Ardley, a small amplitude residual is seen on the difference section and is attributed to low amplitude residual migration noise.

The time-lapse processed P-wave VSP section for Line 1 is shown in Figure 5.10. As expected, the VSP data show higher bandwidth than the surface seismic data, particularly if we examine the Cardium event amplitude response which is stronger in the VSP data compared to the surface seismic data. On the difference section, there is an observed amplitude and time differences at the Cardium reservoir, even though the VSP data was limited to about $100 \mathrm{~m}$ above the reservoir due to geophone array depth and limited vertical aperture.

To summarize this section, the acquisition and processing of the monitoring program was well executed. There were unavoidable repeatability issues with acquisition where few shots could not be repeated due to new obstacles on site. Also, the increasing activities and changes in near-surface conditions caused some of the nonrepeatability observed in the data. Imaging the Cardium reservoir prior to $\mathrm{CO}_{2}$ injection was a challenge, and even after injecting about 60,000 tonnes of gas, there was almost no seismic response at the reservoir level. From the modeling study, the seismic response was subtle after 


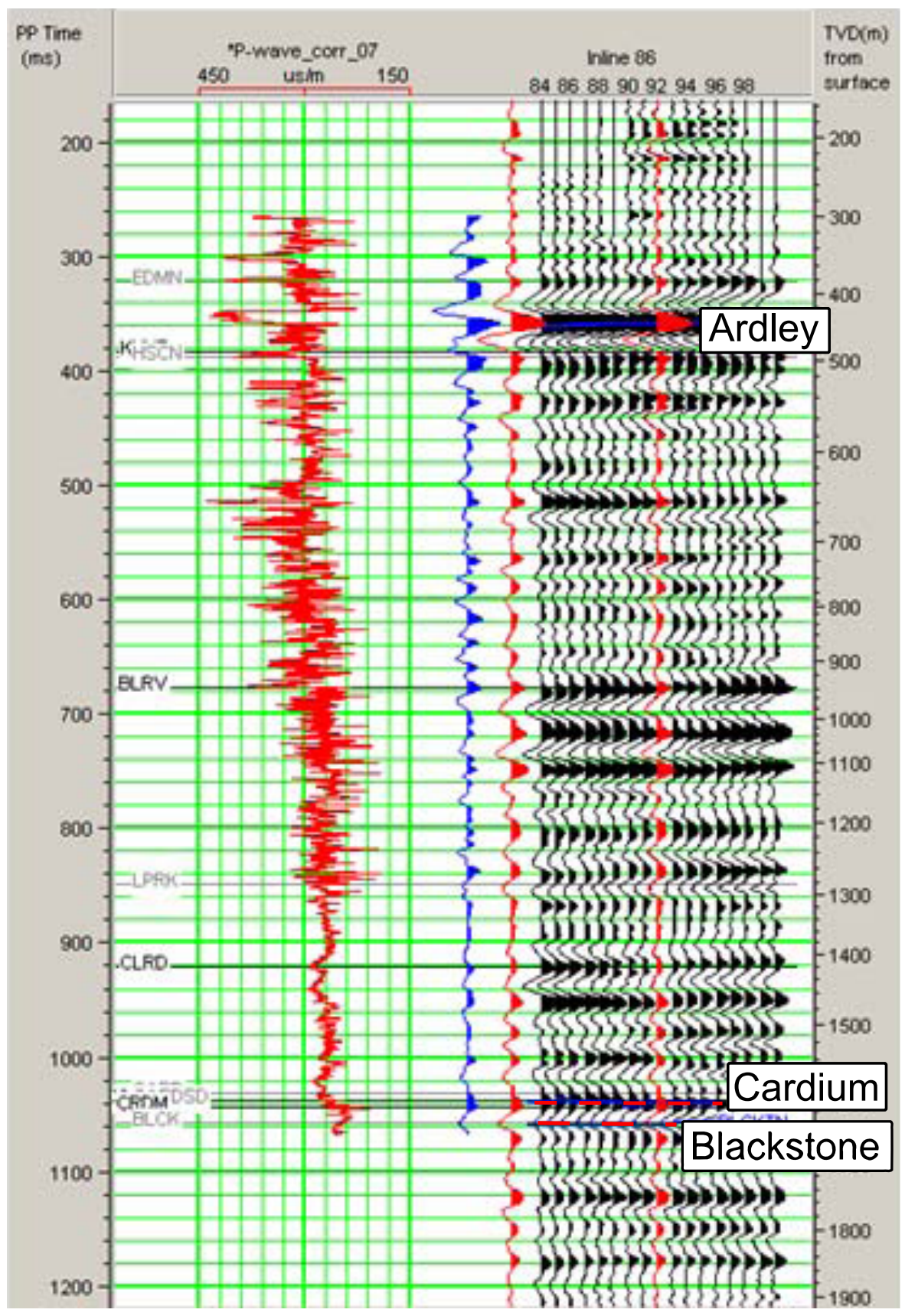

Figure 5.8: P-wave seismic correlation at well 102/7 - 11 - 48 - 9. The blue trace is synthetic seismogram, and the red trace is extracted from the surface seismic at the well location (Chen, 2006). 

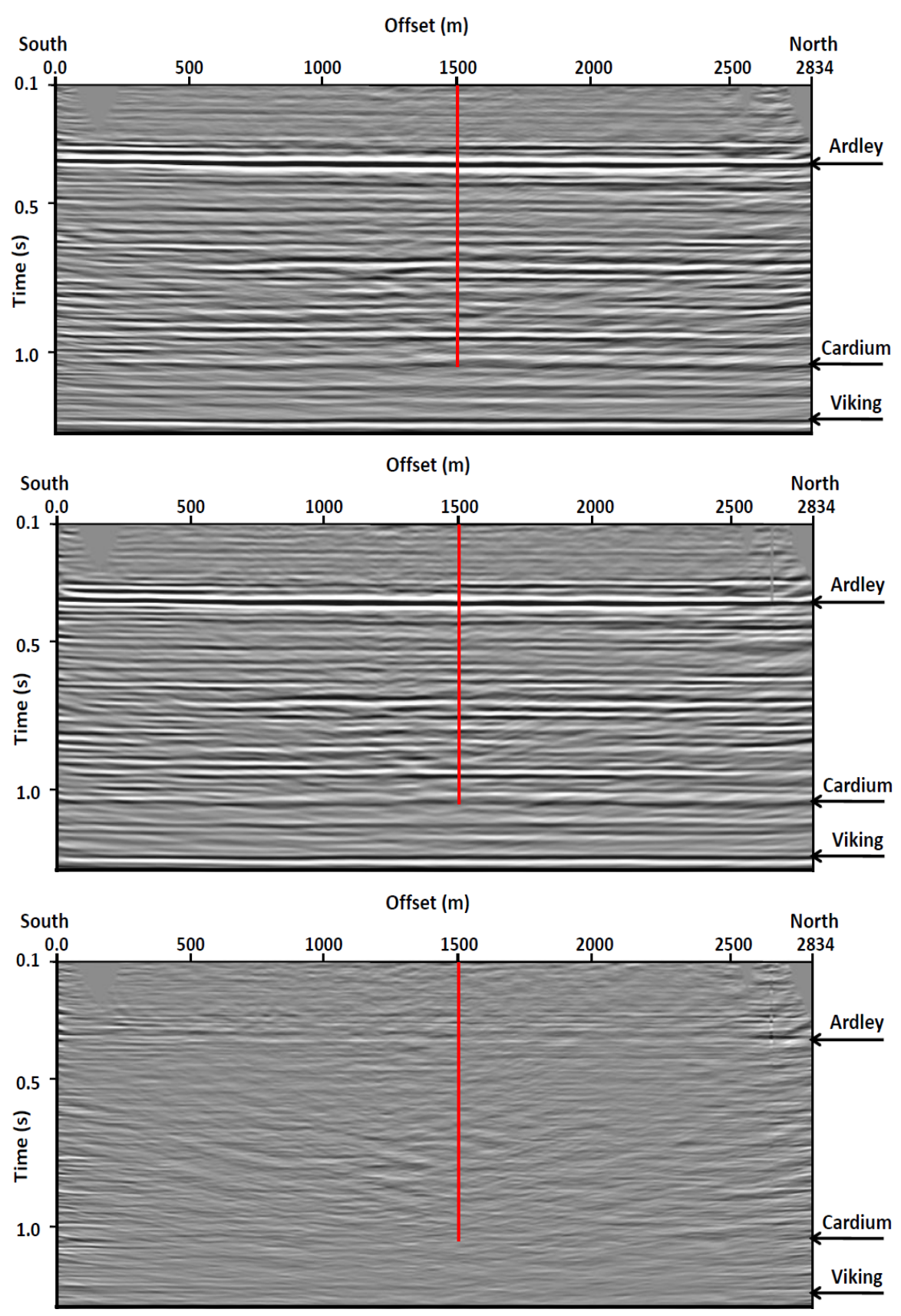

Figure 5.9: Processed P-wave data from Line 1 (Alshuhail et al., 2008). Top is Phase I (baseline), middle is Phase III (monitor) after poststack match filter, and at the bottom is the difference. The red line is the projection of the $102 / 7-11-48-9$ well. 


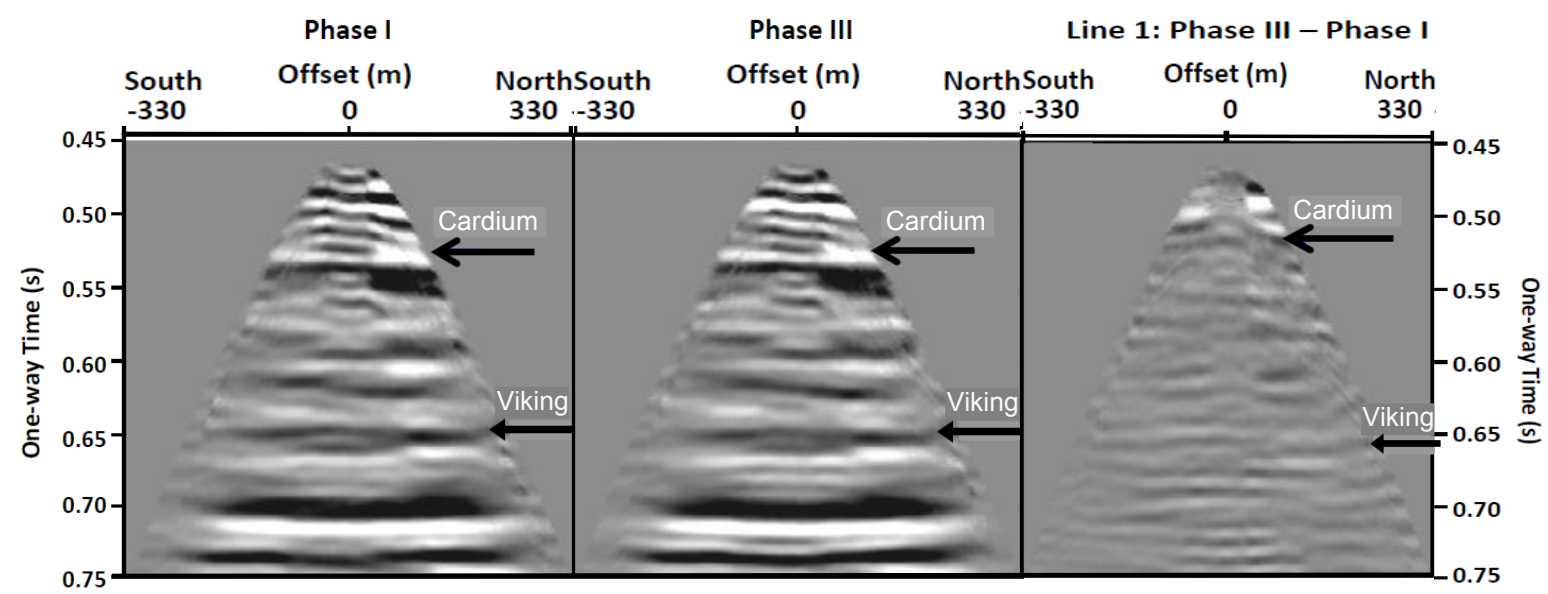

Figure 5.10: Walkaway P-wave VSP data from Line 1 at the observation well (Alshuhail et al., 2008). On the left is Phase I , middle is Phase III, and on the right is the difference.

replacing water and oil with gas. The estimated $4 \%$ decrease in P-wave velocity did not materialize in an observable seismic anomaly. The difference observed on the VSP data was not conclusive and could not be linked to the surface seismic data.

Because of these challenges, which have been stated in several CREWES reports and theses, we decided to reprocess this dataset but for different objectives. The first one is to test the new prestack surface-consistent matching filters on a field dataset with repeatability issues. The second objective is to study which of the known repeatability factors can be resolved with the new algorithm and which can not. There is also another reason for reprocessing this dataset and that is to compare two processing sequences, independent versus simultaneous, as well as to build a processing flow for the new matching filter algorithm. These objectives and the results obtained will be the subject of the next few sections. 


\subsection{Re-processing the Violet Grove data: prestack study}

Line 1 (Figure 5.7) was acquired in 2005 with 77 shot locations and 151 receivers for every shot. In 2007, the same line was acquired with only 71 shot locations but with the same number of receiver locations as the baseline survey (100\% receiver locations repeatability). In the monitor survey, there were 6 shots that were not possible to repeat due to activities on site. There were also 4 shot records acquired in the monitor line that were not acquired in the baseline survey. The total common shot locations in both surveys were 67 shots or about $87 \%$ of the baseline survey shot locations were repeated successfully (Figure 5.11). In this study, we focus on the matched geometry between the two surveys of Line 1.

We will begin by presenting the initial processing steps required for conditioning the data prior to deconvolution and velocity analysis. Then we discuss the two processing strategies, parallel and simultaneous, as well as details of the SCMF computations, including the filter window and length, the four components of the matching filters, and the results after applying the filters.

\subsubsection{Pre-processing steps}

Following the geometry match between the baseline survey and the monitor survey, we examine a shot record from the middle of the line (Figure 5.12). We observe that surfacewaves dominate the shot record and only hints of primary events can be seen below $\sim 1$ second of data on the far-offset traces. We also observe random noise in both surveys that is likely to be nonrepeatable. The difference plot between the baseline and the monitor survey shows that much of the linear noise has been canceled out, but random noise and some primary reflectors appear in the residual plot. To measure the repeatability, we compute the NRMS error, discussed in Section 2.4, for every sample in both shot records

and convolved the result with a $50 \mathrm{~ms}$ smoother. Figure 5.12(d) illustrates high error, 

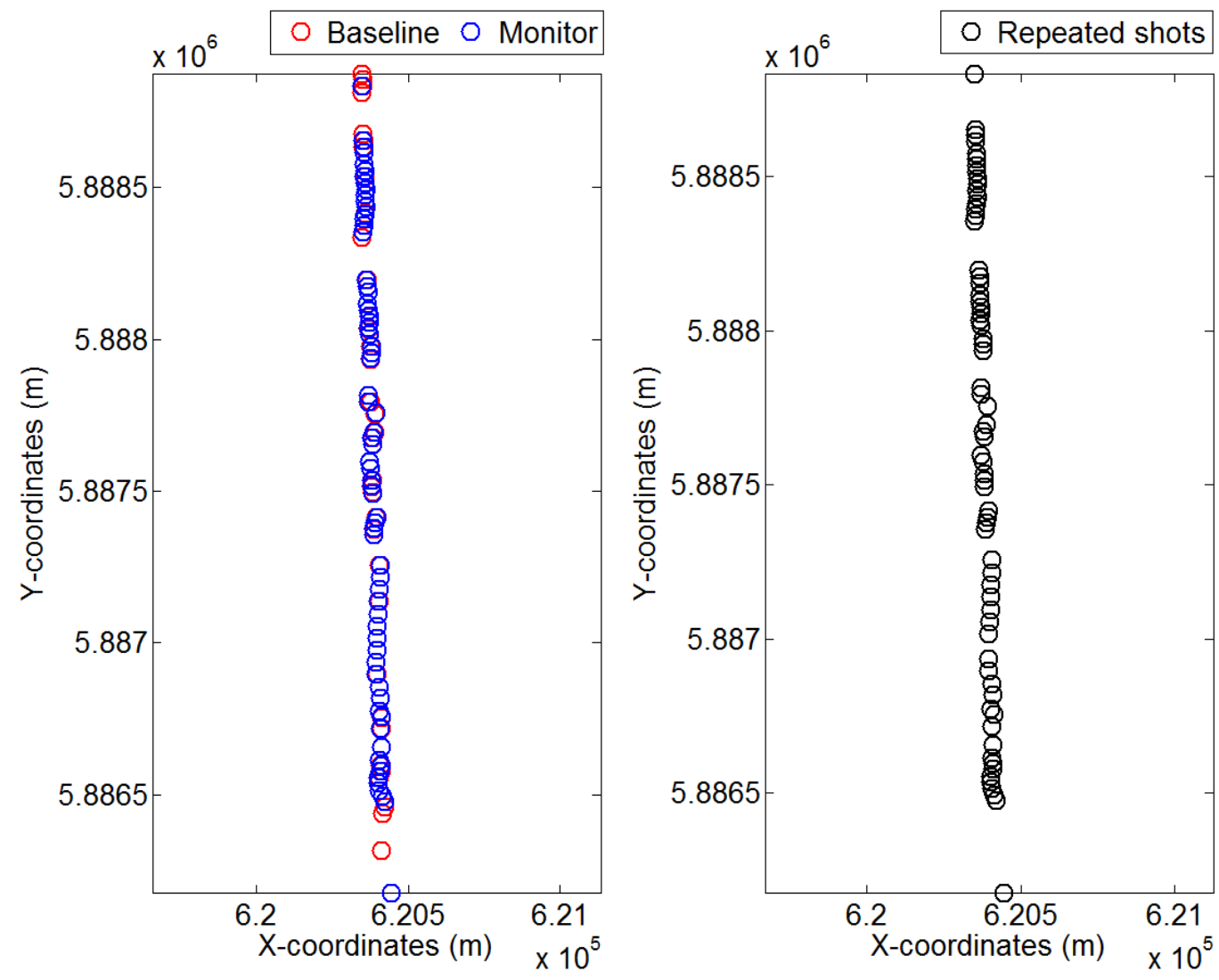

Figure 5.11: Line 1 shot location for the baseline survey, in red, and the monitor survey, in blue, displayed in the left panel. Only the common shot locations were processed for time-lapse as shown in the right panel. 
in red color, dominating the section, except in parts of the section that are masked by surface waves. There is a good repeatability of surface waves but poor repeatability of reflected data and random noise.

The data shown in Figure 5.12 have a 500 ms window AGC for display only. The data require an amplitude correction to compensate for energy loss with depth. The amplitude correction used was a simple time raised to a power of 2.5 as

$$
g(t)=t^{2.5}
$$

where $g$ refers to gain correction and $t$ is time. We have experimented with several power values but 2.5 gave the best result. Following the amplitude adjustment, manual trace edits were required for few of the traces that displayed as anomalous on some of the shot records. The edited traces were zeroed out in both surveys. The linear noise (surface waves, first arrivals, ...etc.) was attenuated using radial trace filtering (Henley, 2003) targeting specific dips with estimated velocity trends and a specific time origin. This is a cascaded process where linear noise trends are estimated first, then subtracted from the data through a least-squares method.

Figure 5.13 is the result after spherical divergence correction and linear noise attenuation. The baseline survey shows higher amplitude and different random noise (examples are indicated by the green arrows) than the monitor survey. The difference between the baseline and the monitor is shown in Figure 5.13(c) and illustrates clear nonrepeatable noise and primaries in the data that are not related to the injected $\mathrm{CO}_{2}$ gas. The high amplitude noise close to the near offsets $(0 \mathrm{~m}$ to $+/-500 \mathrm{~m})$ have low dips and are difficult to attenuate using dip filtering techniques. The presence of noise makes investigating the Cardium reservoir on prestack data a difficult task, especially when it has a low impedance response. The low seismic response of the Cardium reservoir can hardly be distinguished from the high amplitude noise, even on the monitor survey after the gas 


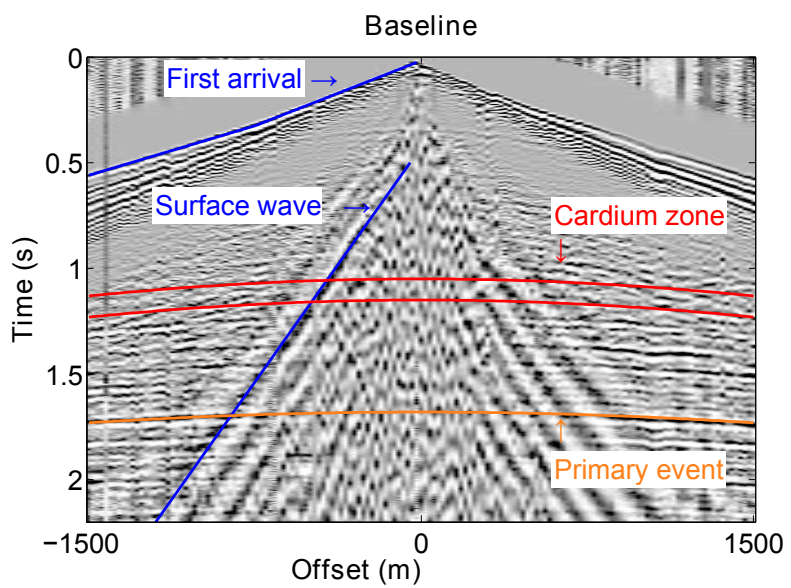

(a)

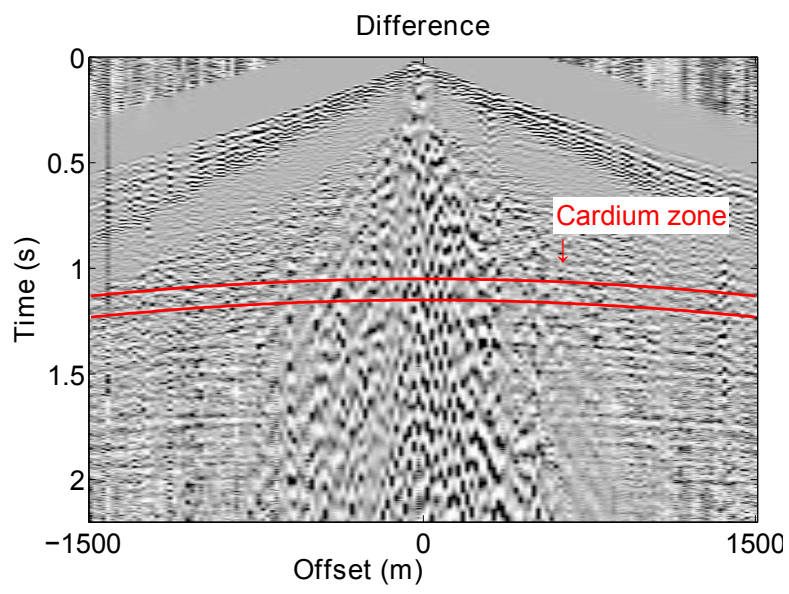

(c)

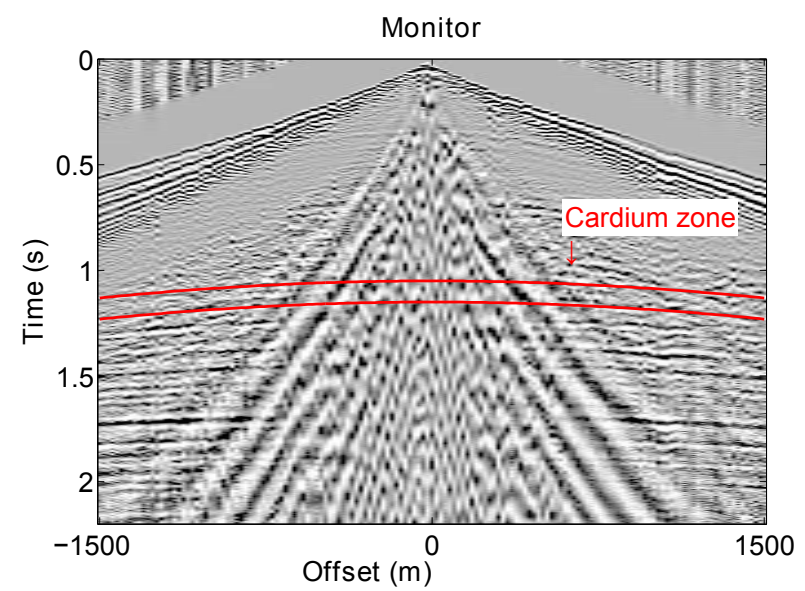

(b)

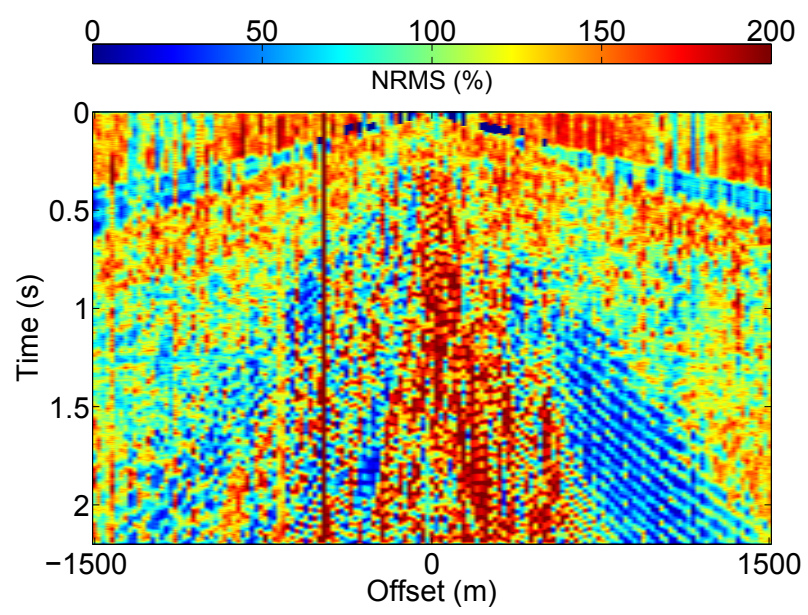

(d)

Figure 5.12: A raw shot record from the middle of Line 1. (a) shows the baseline, (b) shows the monitor, (c) shows the difference, and (d) shows the computed NRMS error. Red and yellow color mean high NRMS errors whereas blue means low NRMS errors. An AGC filter with $500 \mathrm{~ms}$ length is applied for display purposes. Plots (b) and (c) have the same amplitude scaling as plot (a). 
injection, where an increase in amplitude response is expected.

Above the Cardium reservoir, the prestack data lack strong coherent events, with the exception of the Ardley Coal zone. This shallow event can be used for designing the surface-consistent matching filters calculation. However, we decided not to use it in this study for two reasons: 1) the limited offset extent (or low fold) which makes it unfavorable for offset and midpoint filters, and 2) the high noise level generated from the source and caused by proximity to the weathering layer.

Because of the weak seismic response of the Cardium reservoir, even after $\mathrm{CO}_{2}$ injection, and the difficulty to distinguish it from the the high amplitude residual noise in the data, we are modifying the objective of re-processing this data set. The new focus is to minimize the nonrepeatability observed in the difference between the two datasets utilizing the SCMF algorithm. We choose a window below the Cardium reservoir due to higher signal-to-noise ratio and more reflected events. The minimization process will be through the development of a set of surface-consistent matching filters designed from the baseline and the monitor surveys.

\subsubsection{Parallel processing}

In this section, we process the baseline survey and the monitor survey independently. The processing flow in Table 5.4 illustrates how the P-wave data were processed, as well as some of the key parameters. This processing flow is fairly similar to that discussed in Table 5.3.

As discussed in Chapter 4, an independent processing scheme without SCMF will estimate independent source and receiver wavelets and static corrections for each survey. Surface-consistent deconvolution may result in both datasets having similar wavelets, but this is not guaranteed, since there is different random noise in each dataset. Moreover, the long-wavelength error characteristic of surface-consistent methods will not be common if 


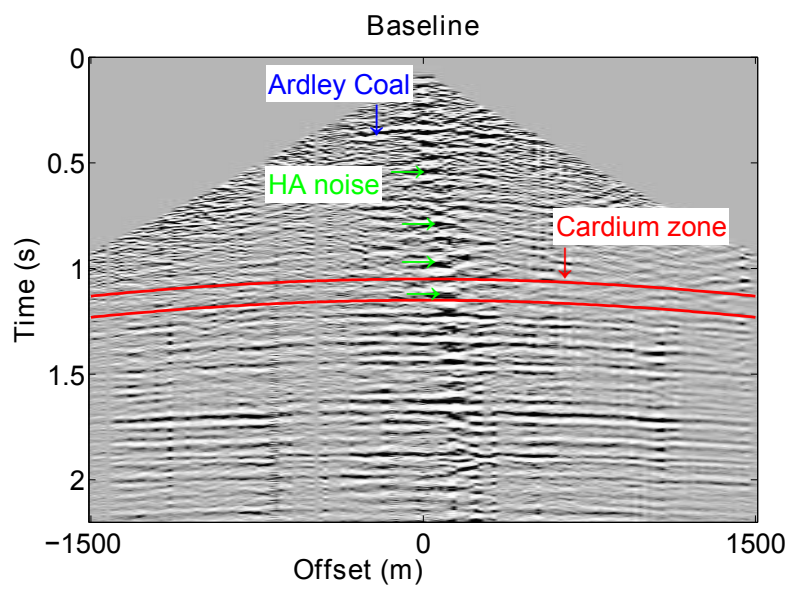

(a)

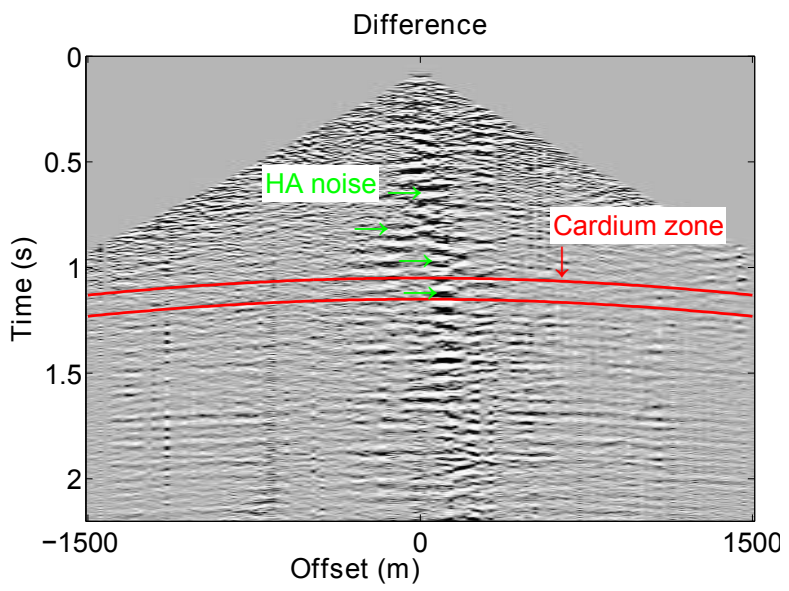

(c)

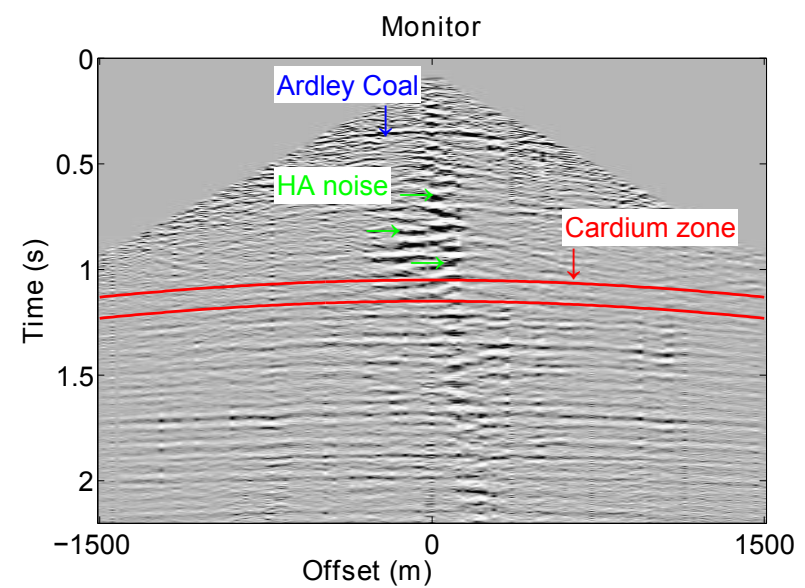

(b)

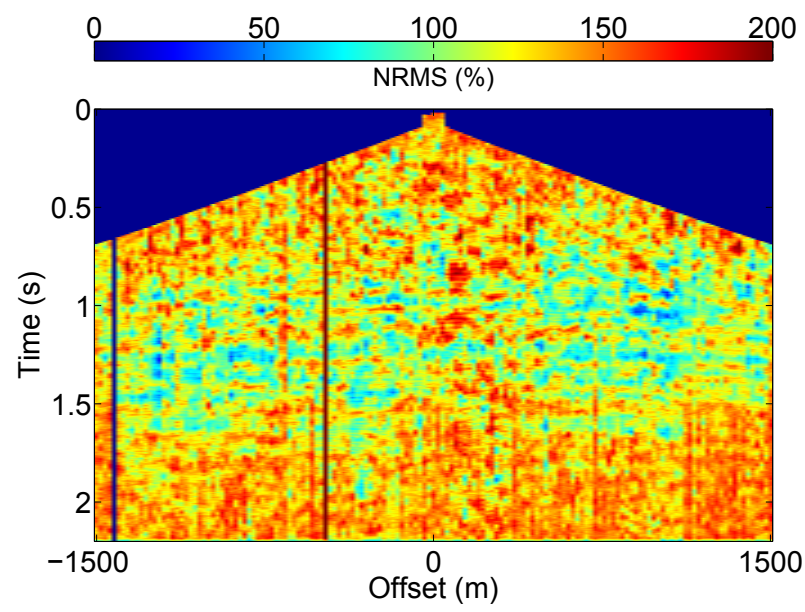

(d)

Figure 5.13: Data after amplitude recovery compensation and linear noise attenuation. (a) shows the baseline survey, (b) shows the monitor survey, (c) shows the difference, and (d) shows the NRMS error. Note the clear difference in amplitude strengths between the base and the monitor as well as the nonrepeatability of the high amplitude (HA) noise indicated by green. Plots (b) and (c) have the same amplitude scaling as plot (a). 
both lines are processed independently.

A minimum phase surface-consistent spiking deconvolution (SCSD), with 120 ms operator length and $0.1 \%$ prewhitening, was computed independently for each line. The SCSD was computed over a window extending from about $200 \mathrm{~ms}$ to about $2500 \mathrm{~ms}$. Then four-term surface-consistent amplitude corrections (SCAC) are computed using a deep window (light blue window in Figure 5.14) with good coherent events. Figure 5.14 shows the result after SCSD and SCAC. The difference between the baseline and the monitor line has been reduced slightly compared to that shown in Figure 5.13, particularly where coherent events are present between 1 and 2 second. The high amplitude noise near the zero-offset has been attenuated, but random noise increased slightly after deconvolution, as expected. This is reflected in the computed NRMS error (Figure 5.14(d)) when compared to the NRMS error presented in Figure 5.13(d).

Table 5.4: Parallel processing flow of Line 1 P-wave surface seismic data set.

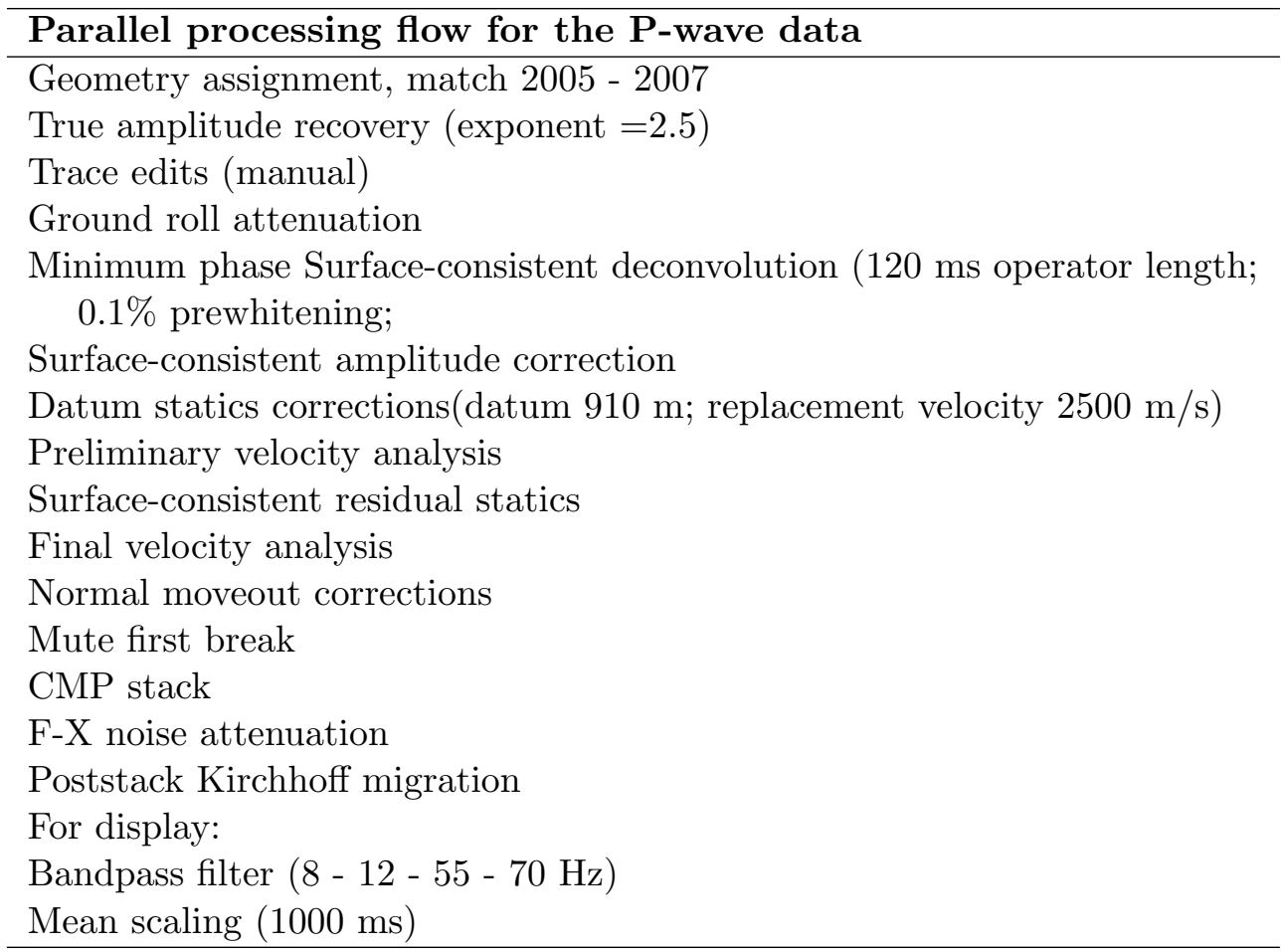

For every survey, deconvolution and amplitude adjustments are followed by a pre- 
liminary velocity analysis, a first pass of surface-consistent residual static corrections, a final velocity analysis, then a second pass of surface-consistent residual static corrections. Figure 5.15 shows the final velocity analysis of CMP 135 (middle of the line) from the baseline survey. Our careful analysis of the velocity at the Cardium level did not boost its seismic response.

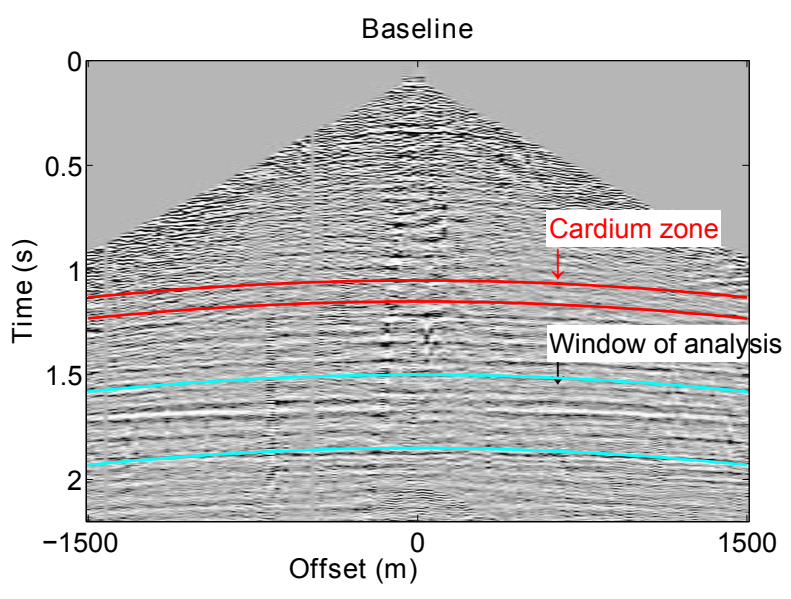

(a)

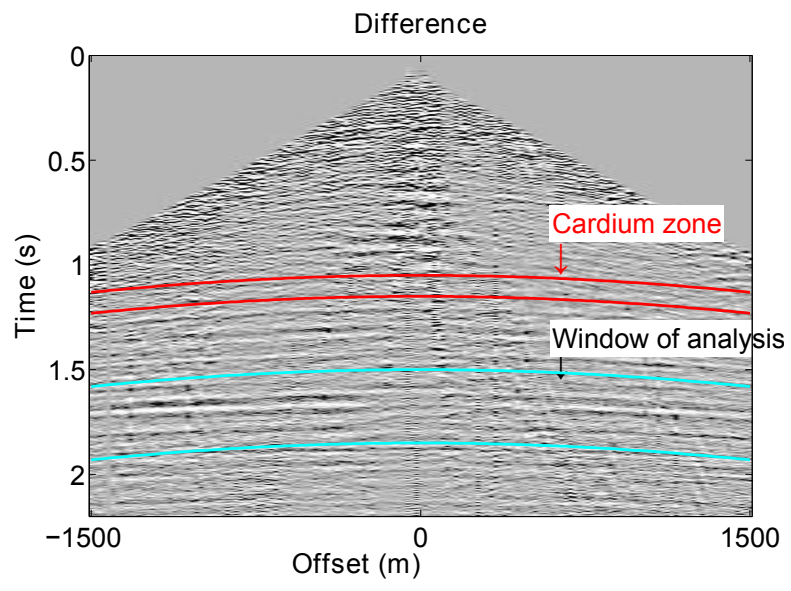

(c)

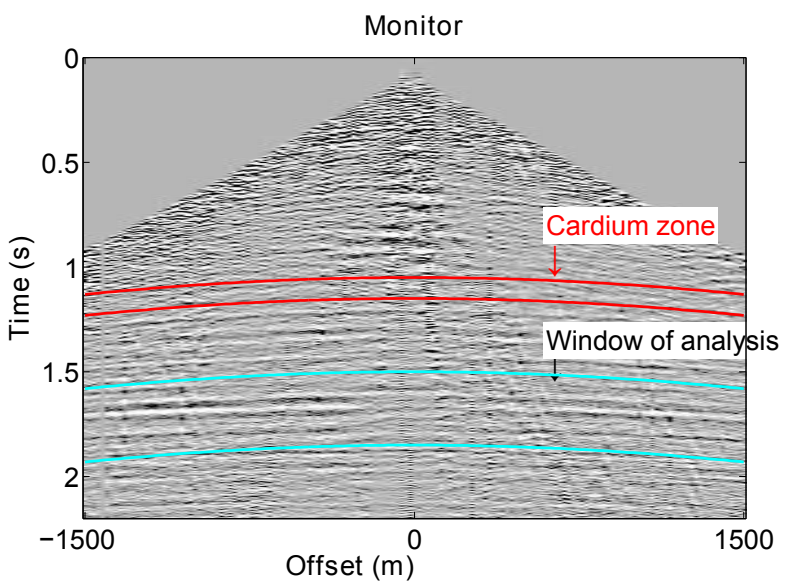

(b)

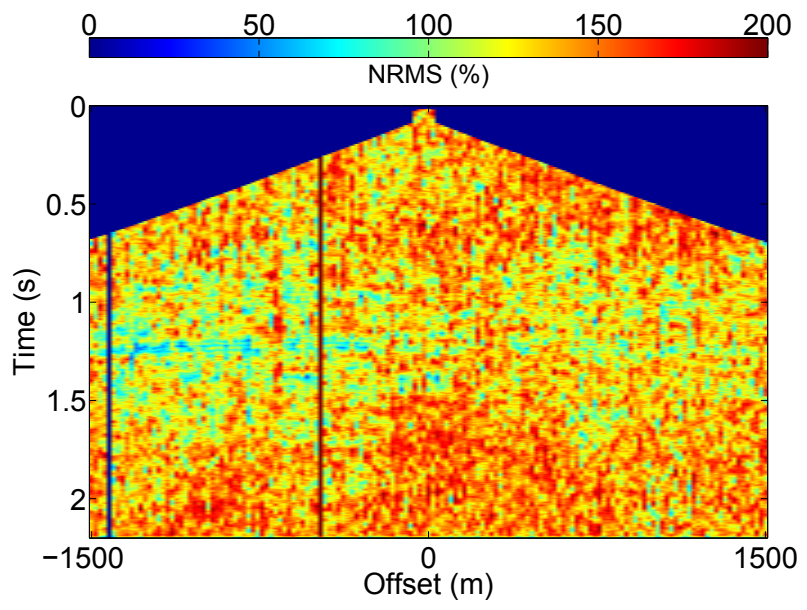

(d)

Figure 5.14: The baseline shot (a) and the monitor shot (b) after independent SCSD and independent SCAC. The difference is shown in (c) and the NRMS error is in (d). Plots (b) and (c) have the same amplitude scaling as plot (a). 


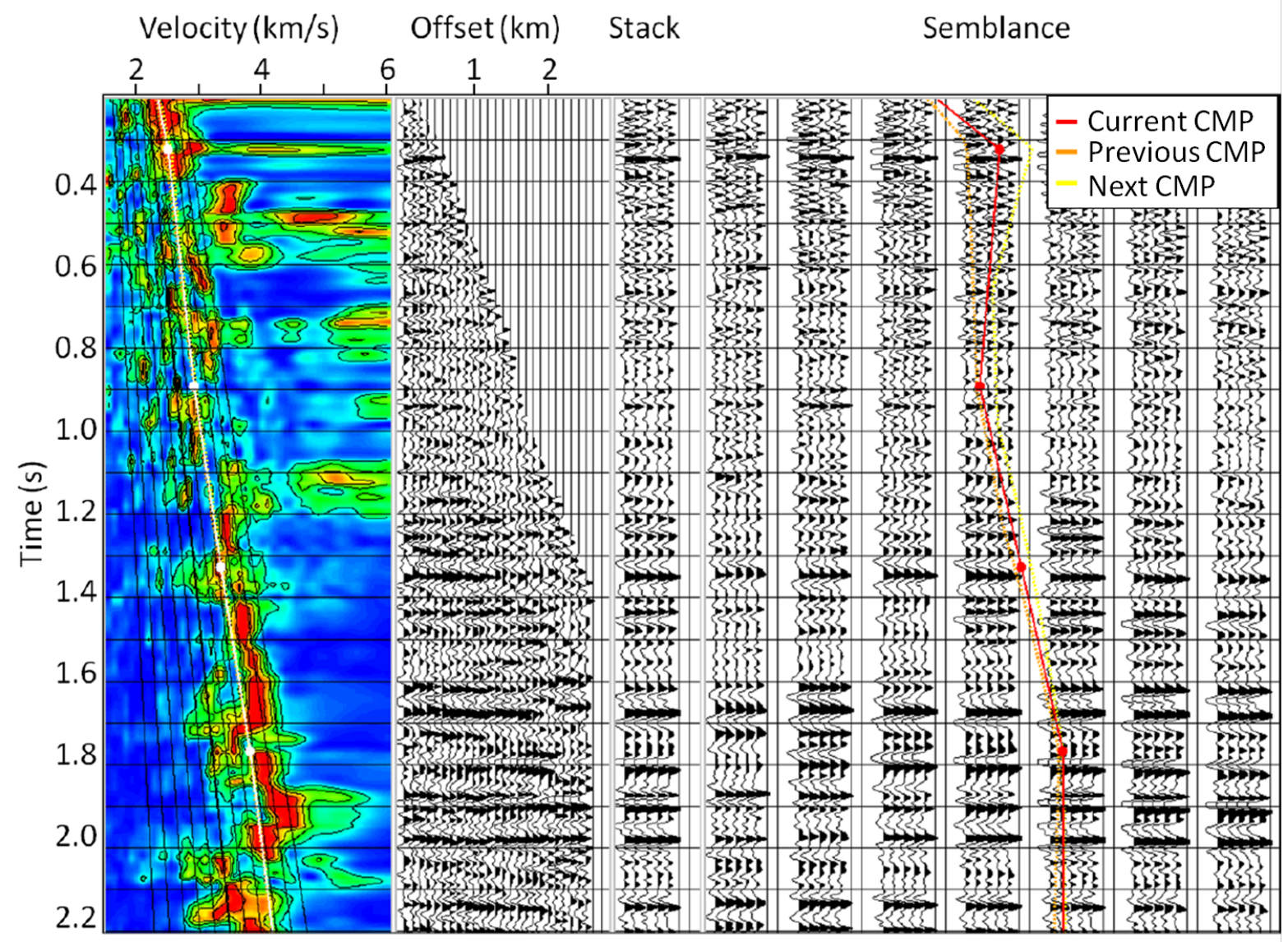

Figure 5.15: Final velocity analysis for CMP 135. 


\subsubsection{SCMF}

In this section, we will compute the surface-consistent matching filters (SCMF) using the baseline and the monitor surveys after linear noise attenuation and spherical divergence correction. The SCMF will be computed so that the monitor line is matched to the baseline. We compute the four components SCMF using equation 3.14. Similar to what has been presented in the synthetic datasets, the geometry matrix is overdetermined (more equations than unknowns) and underconstrained (deficient in the number of independent equations available to solve for the unknowns).

The computed SCMF is centered on a strong reflector below the Cardium Formation (window of analysis in Figure 5.14). The window length is $300 \mathrm{~ms}$ and the filter length is $75 \%$ of the trace length. We begin by crosscorrelating traces from the monitor survey and the baseline survey and align them, based on maximum crosscorrelation. After that, we compute trace-by-trace time-domain match filters. Each time-domain match filter is transformed to the frequency domain, which is followed by an independent surface-consistent decomposition for each frequency. This is followed by an inverse Fourier transform of the solution to time-domain. We also decompose the time shifts

computed to align the traces into a surface-consistent set (Figure 3.9). The output of the algorithm is a set of surface-consistent matching filters with associated surface-consistent time-shifts. Both outputs are used to match the monitor survey to the baseline survey in the least-squares sense.

The source and receiver-components of the surface-consistent filters are illustrated in Figure 5.16 and similarly the two subsurface-consistent components are shown in Figure 5.17. The computed source and receiver strengths (the time-average filter magnitude) will not be used and only displayed as a form of quality control. We notice a reasonable correlation between each of the component's strength and its fold (Figure 5.23), particularly for the offset term. 
In addition to the four-component filters, we decomposed the time-shifts used to align the traces before computing the trace-to-trace match filter. Figure 5.18 illustrates the two term, source and receiver, surface-consistent time-shifts. The four-term SCMF and the two-term surface-consistent time-shifts are convolved with the monitor survey in order to match it to the baseline.

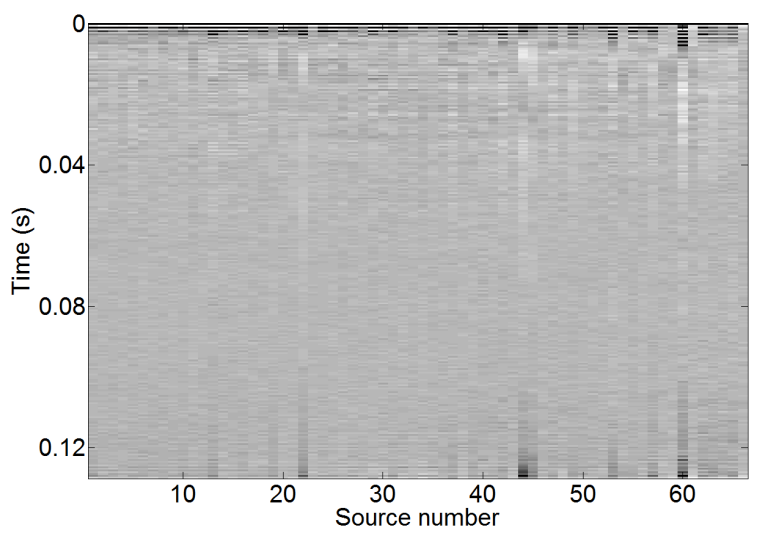

(a)

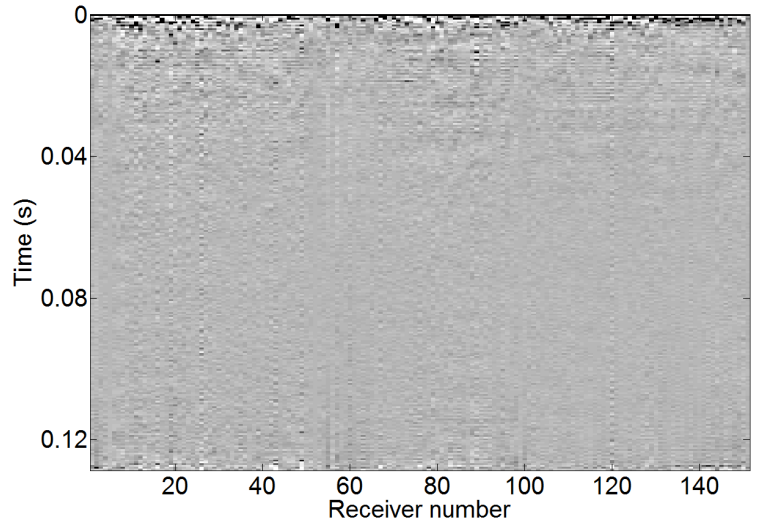

(c)

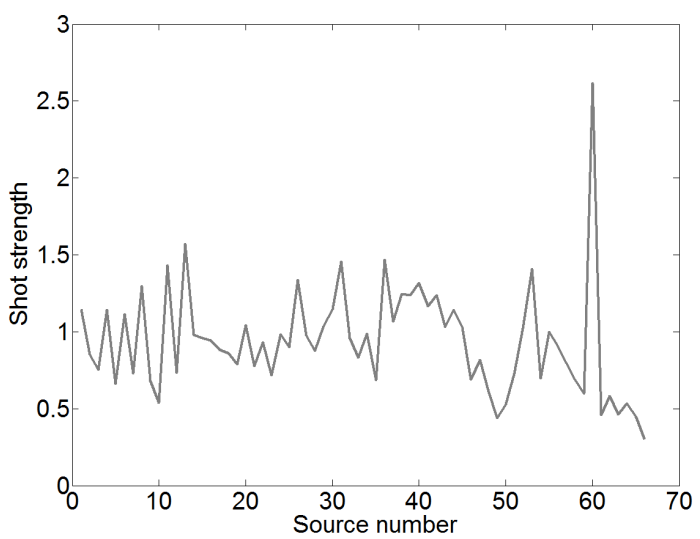

(b)

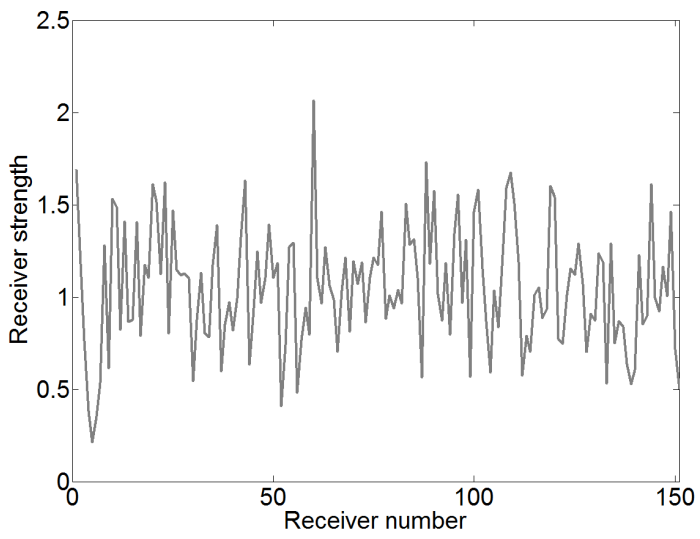

(d)

Figure 5.16: Computed source filters shown in (a) and the time-average of the source filters magnitude in (b). In (c) is the receiver filters and their mean magnitude is in (d).

Figure 5.19 shows a baseline shot record and a monitor shot record after applying the four-term SCMF. The difference, particularly that of primary events observed between $1.5 \mathrm{~s}$ and $2.0 \mathrm{~s}$, is minimized in the new difference in Figure 5.19(c). The residual is mostly 


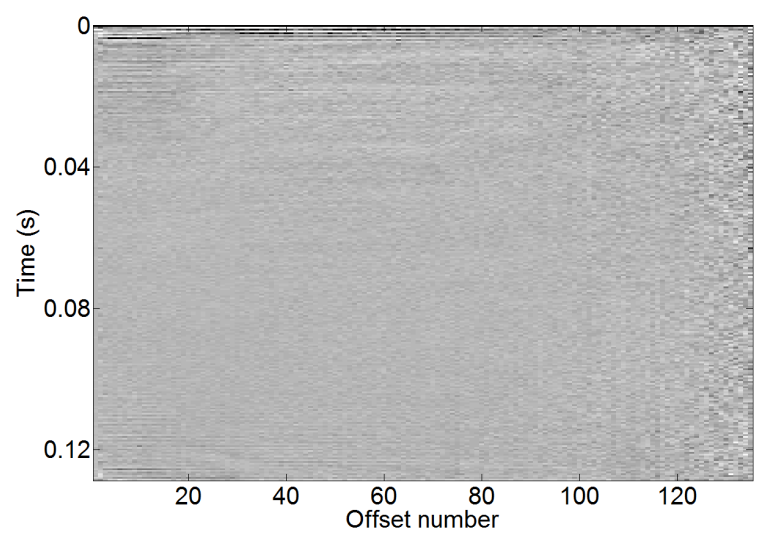

(a)

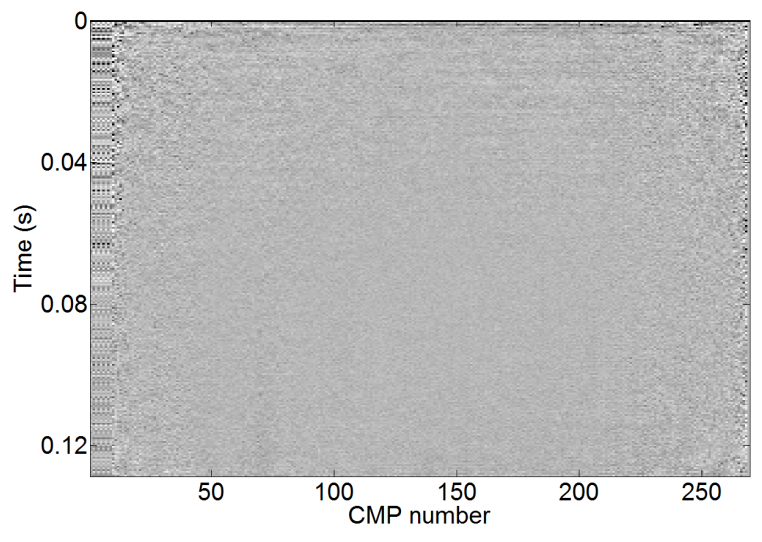

(c)

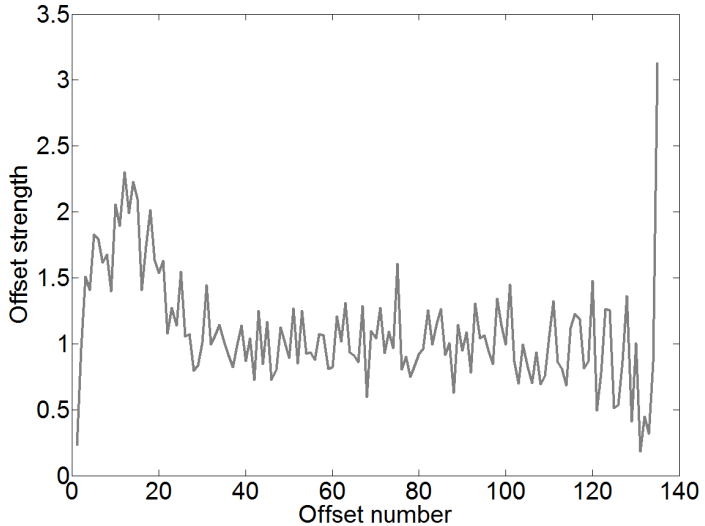

(b)

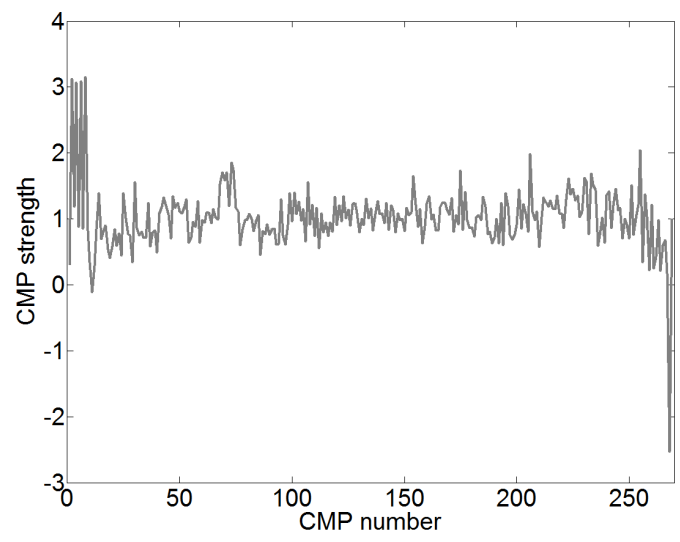

(d)

Figure 5.17: Computed offset filters shown in (a) and their mean magnitude for each offset in (b). (c) shows the midpoint filters, and their mean magnitude is in (d). 


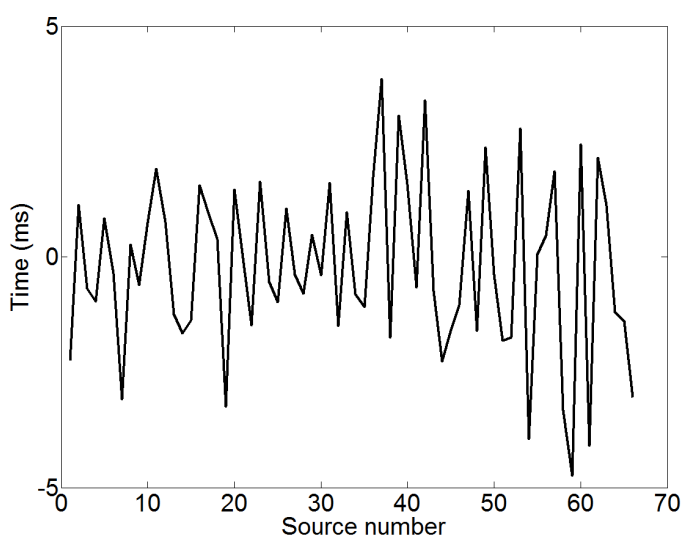

(a)

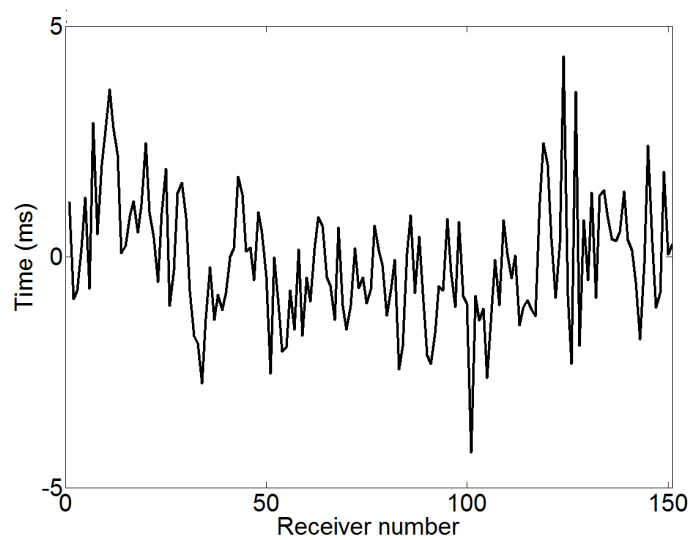

(b)

Figure 5.18: The surface-consistent time-shifts for the source terms (a) and the receiver terms (b).

random noise that is difficult to model in a surface-consistent algorithm. We decided to leave this random noise in the prestack data, but we expect that in the process of CMP stacking that much of the noise will be canceled out.

Figure 5.20 is a zoom into the SCMF window for the same shot record illustrated in Figures 5.13 and 5.19. The SCMF corrected for the amplitude difference between the baseline and monitor surveys as well as time and phase shifts. There is a minimum residual that is related to primary event. Much of the high amplitude in the middle of the shot is random noise.

\subsubsection{Simultaneous processing}

The SCMF should equalize the source and receiver wavelets and the statics of the monitor survey to that of the baseline survey. The output after applying the SCMF to the monitor data is a new matched monitor survey with matched source and receiver terms as well as the two subsurface terms (offsets and midpoints). Our modified simultaneous processing discussed in Chapter 4 is possible at this stage. The baseline and the matched monitor datasets are then merged for all subsequent surface-consistent processes. These included 


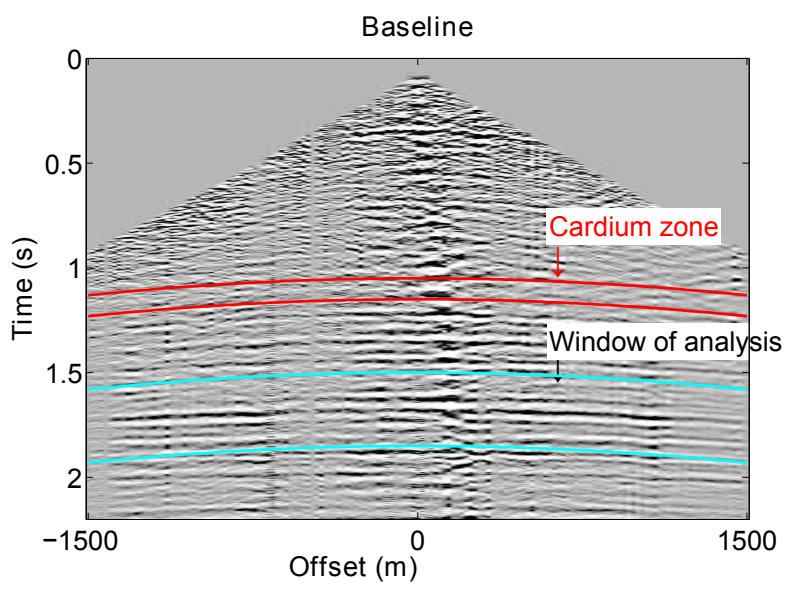

(a)

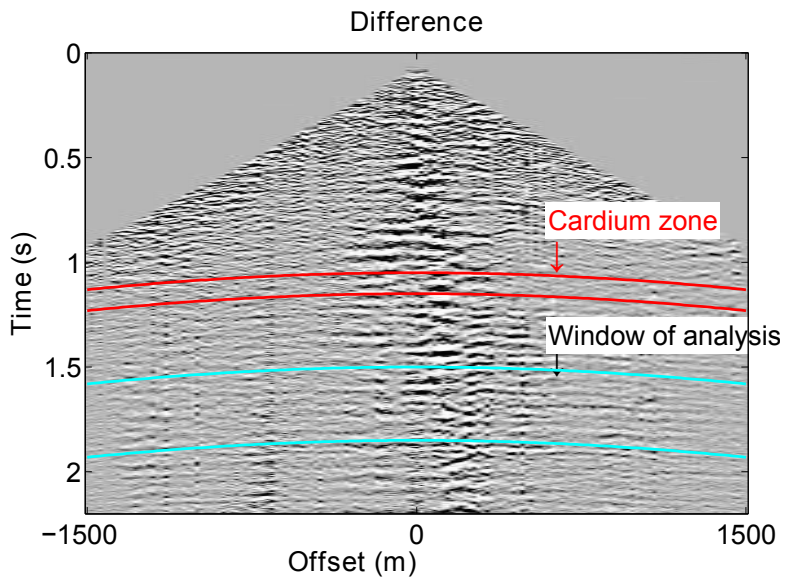

(c)

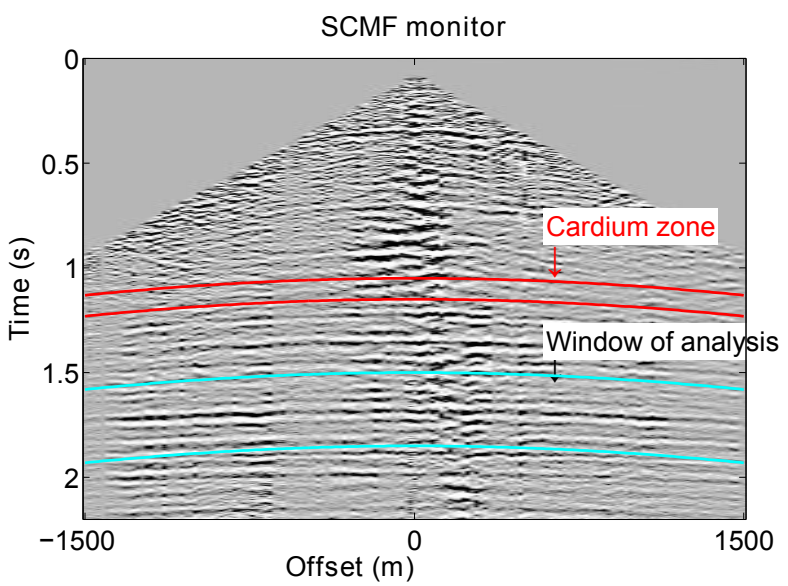

(b)

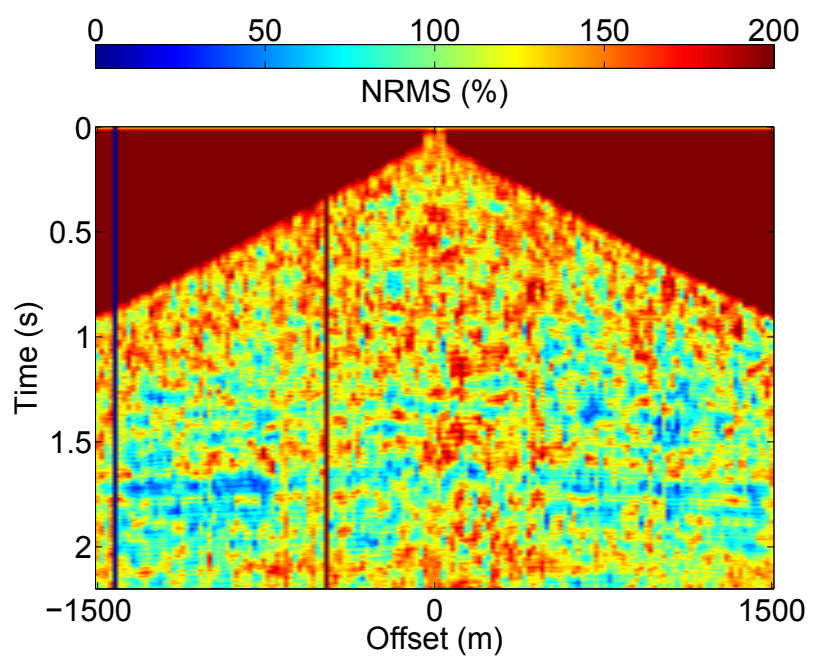

(d)

Figure 5.19: The baseline shot (a) and the monitor shot after applying the four-components SCMF (b). The difference is shown in (c) and the NRMS error is in (d). Note that the difference observed in Figure 5.13(c) is minimized after applying the SCMF, with the exception of the near-offset noise. Plots (b) and (c) have the same amplitude scaling as plot (a). 


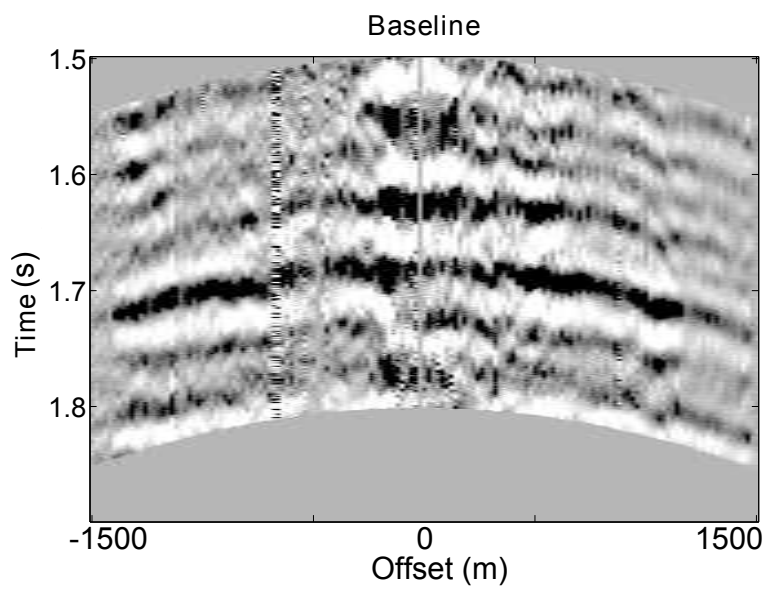

(a)

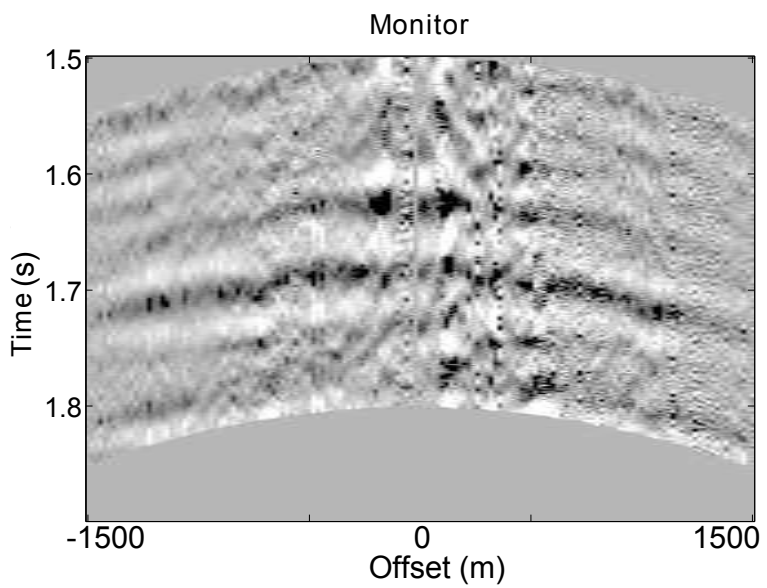

(b)

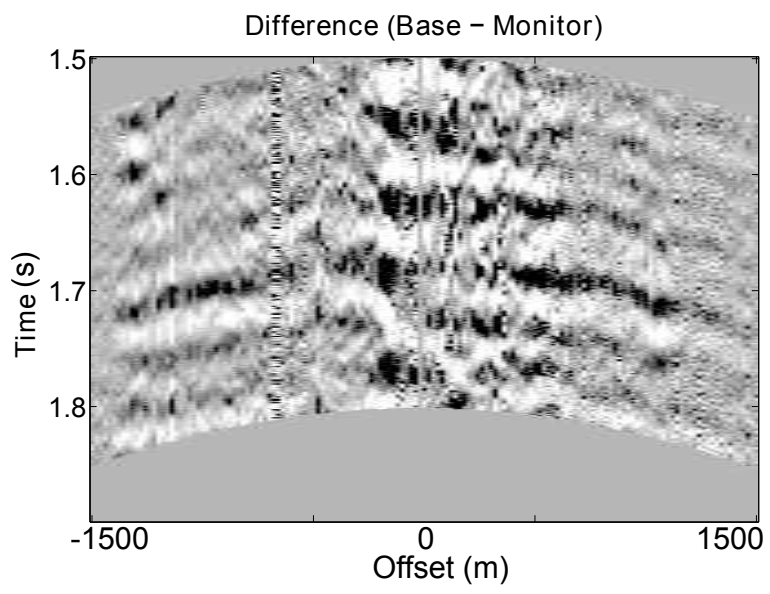

(d)

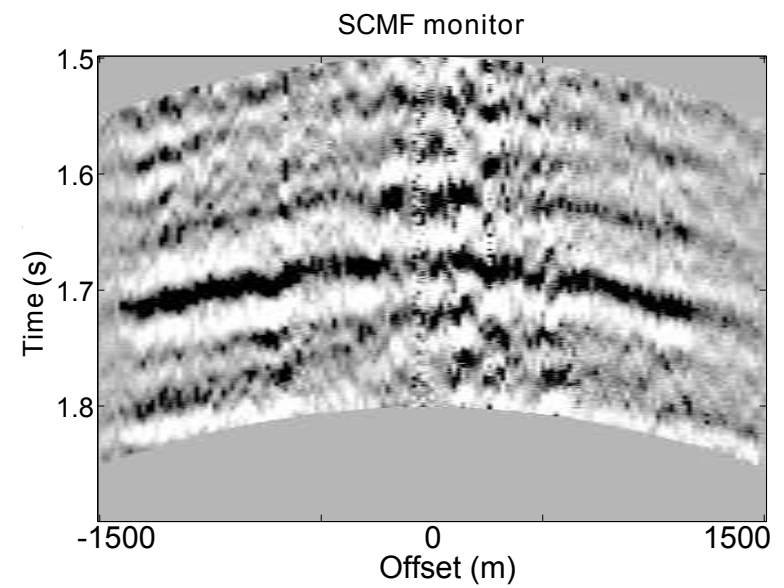

(c)

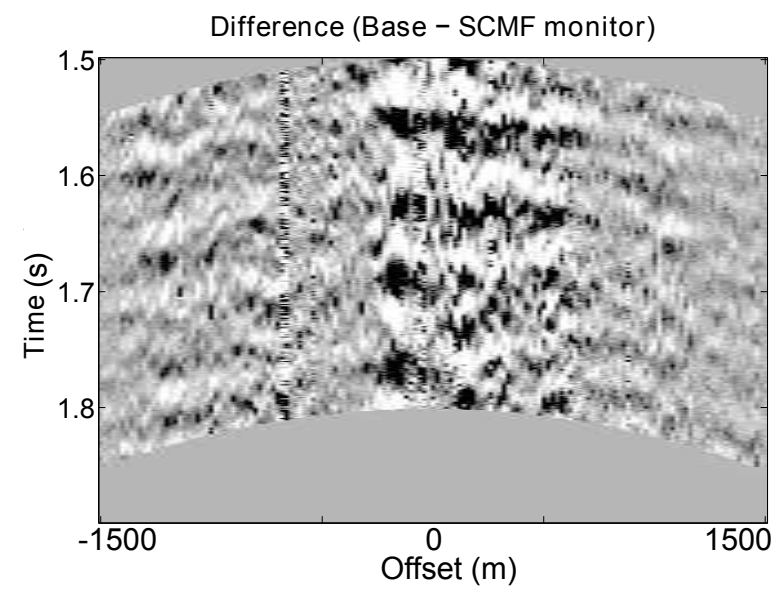

(e)

Figure 5.20: A zoom on the match filter window for the baseline (a), monitor (b), SCMF monitor (c), difference before SCMF (d), and difference after applying SCMF (e). All plots have the same amplitude scaling as the baseline. 


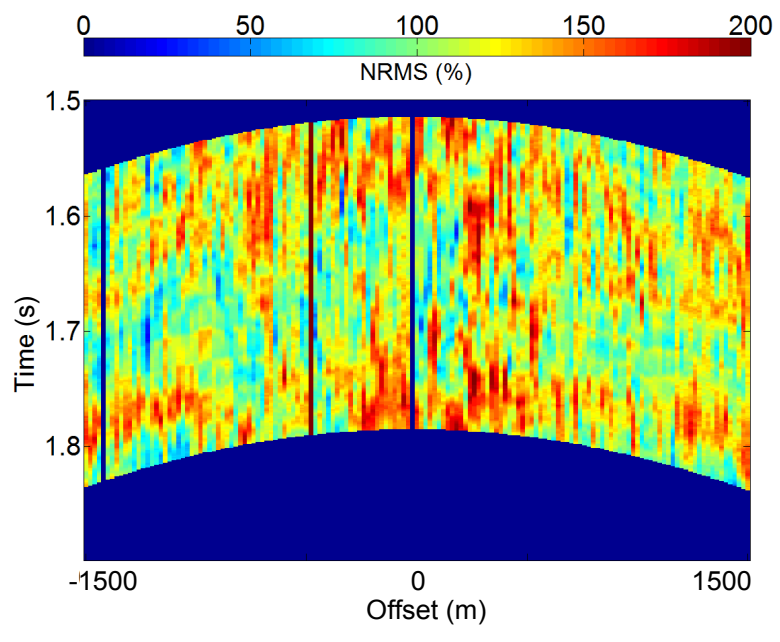

(a)

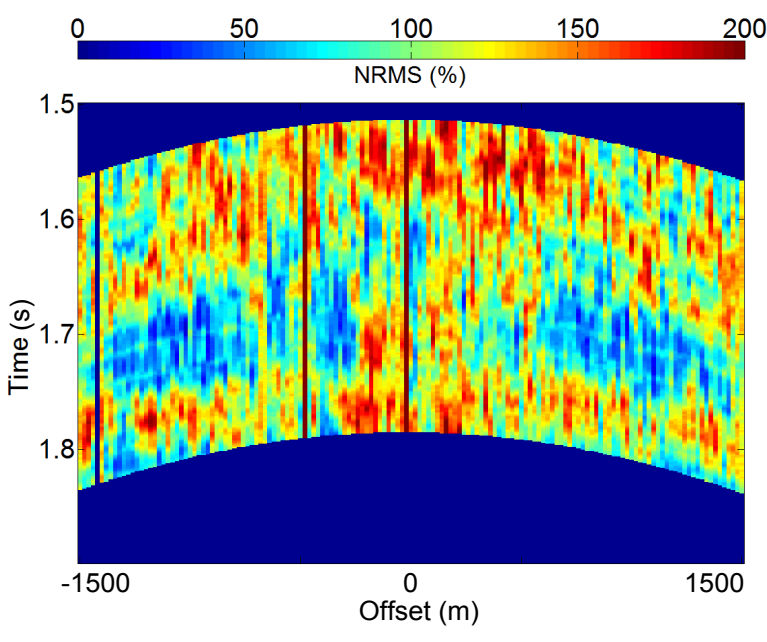

(b)

Figure 5.21: (a) shows the NRMS error computed from the baseline and the monitor without SCMF, and (b) shows the NRMS error after applying SCMF to the monitor survey.

a pass of surface-consistent spiking deconvolution (SCSD), a pass of surface-consistent amplitude correction, and two passes of surface-consistent residual statics (Table 5.5).

Table 5.5: Simultaneous processing flow of Line 1 P-wave surface seismic data set.

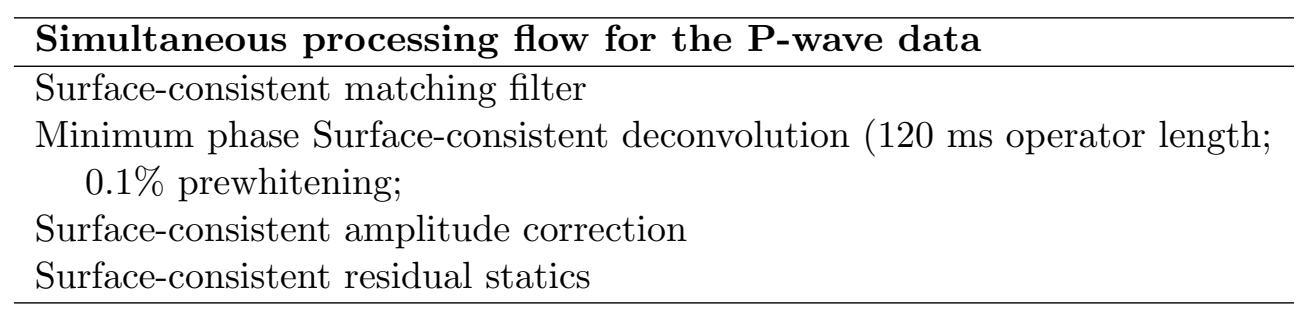

The process for merging the two datasets for SCSD starts by sorting the baseline data into the source domain, then constructing a single vector for every shot record where all traces are concatenated into a long vector or "supertrace". The same procedure is repeated for all shot records. Similarly, the monitor data are sorted in source domain, then a single vector is formed for every shot record. We merge the datasets by forming a single long vector for every co-located shot. We call each of the supertraces the input seismic which will be used in a time-domain Wiener spiking deconvolution algorithm. 
Similarly, we form a design supertrace for every shot record. The design trace is shorter than the input trace and is extracted from a constant time window, $0.3 \mathrm{~s}$ to $2.3 \mathrm{~s}$. We solve the least-squares system and save the output.

After deconvolving the source term, we store the data and use it as input after sorting it into the receiver domain so that the receiver term can be deconvolved too. A similar process is done for the other two components, offsets and midpoints, respectively.

Figure 5.22 is the result of simultaneous SCSD. This result is slightly noisier but displays a sharper image than that shown in Figure 5.20. NRMS errors are sensitive to random noise, and since random noise is different in both surveys, the NRMS error after simultaneous SCSD has increased slightly.

We follow the simultaneous SCSD with surface-consistent amplitude correction (SCAC). We compute the RMS (root-mean-squares) amplitude for every trace over the same window used for the SCMF. The form of the least-squares system is:

$$
\left[\begin{array}{c}
\mathbf{G}_{b} \\
\mathbf{G}_{m}
\end{array}\right][\mathbf{x}]=\left[\begin{array}{c}
\mathbf{d}_{b} \\
\mathbf{d}_{m}
\end{array}\right]
$$

where $\mathbf{G}_{b}$ and $\mathbf{G}_{m}$ represent the geometry matrix for the baseline survey and the monitor survey, respectively. In this example, both matrices are exactly the same. $\mathbf{x}$ is a vector containing the unknowns, mainly the sources, the receivers, the offsets, and the midpoints. The right-hand-side of equation 5.2 contains the data vector, where $\mathbf{d}_{b}$ represents the RMS amplitude of every trace in the baseline survey, and $\mathbf{d}_{m}$ represents the RMS amplitude of every trace in the monitor survey. The inversion is solved by using the damped leastsquares method:

$$
[\mathbf{x}]=\left[\begin{array}{c}
\mathbf{G}_{b}^{T} \mathbf{G}_{b}+\alpha^{2} \mathbf{I} \\
\mathbf{G}_{m}^{T} \mathbf{G}_{m}+\alpha^{2} \mathbf{I}
\end{array}\right]^{-1}\left[\begin{array}{l}
\mathbf{G}_{b}^{T} \\
\mathbf{G}_{m}^{T}
\end{array}\right]\left[\begin{array}{l}
\mathbf{d}_{b} \\
\mathbf{d}_{m}
\end{array}\right] .
$$

A small $\alpha$ is used to stabilize the inversion (we found that 1 works best). Figure 5.23 shows the decomposed four-term amplitude corrections. The four corrections are directly 


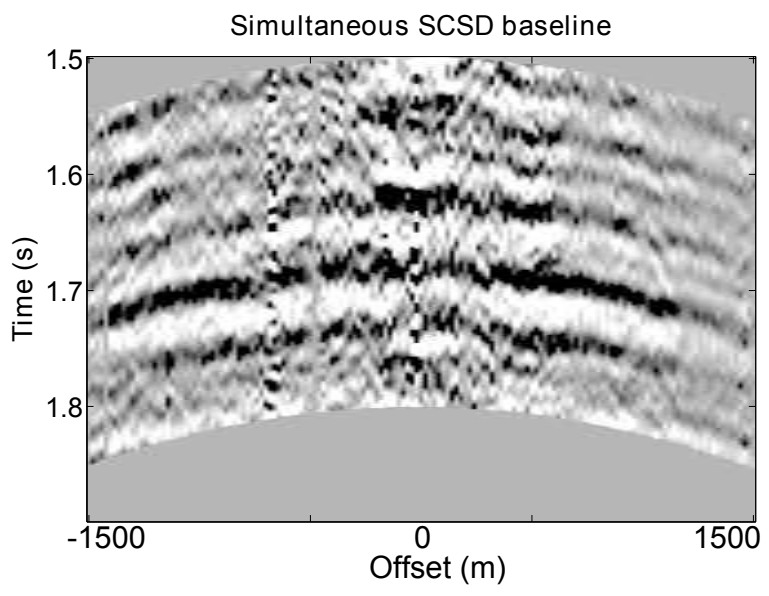

(a)

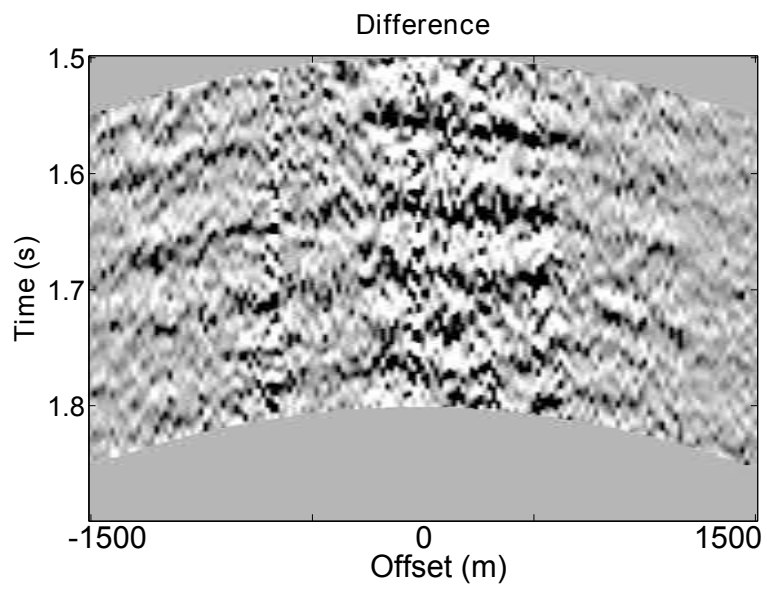

(c)

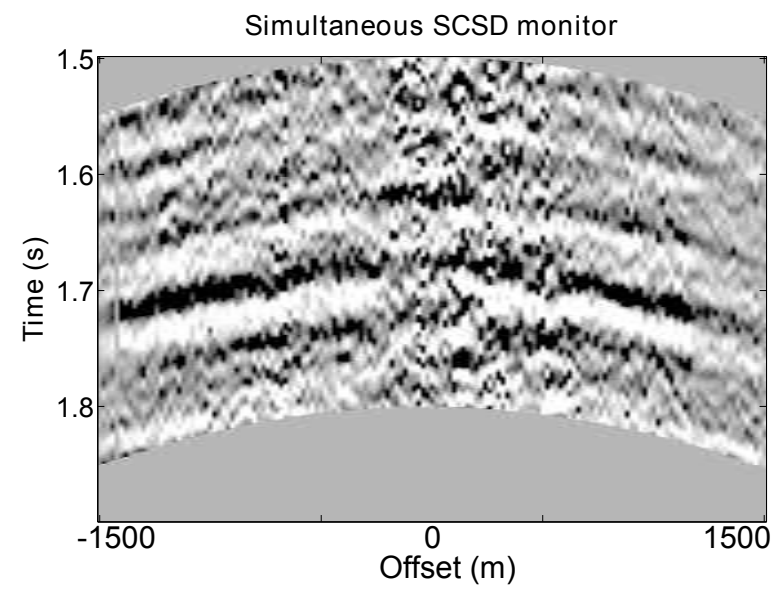

(b)

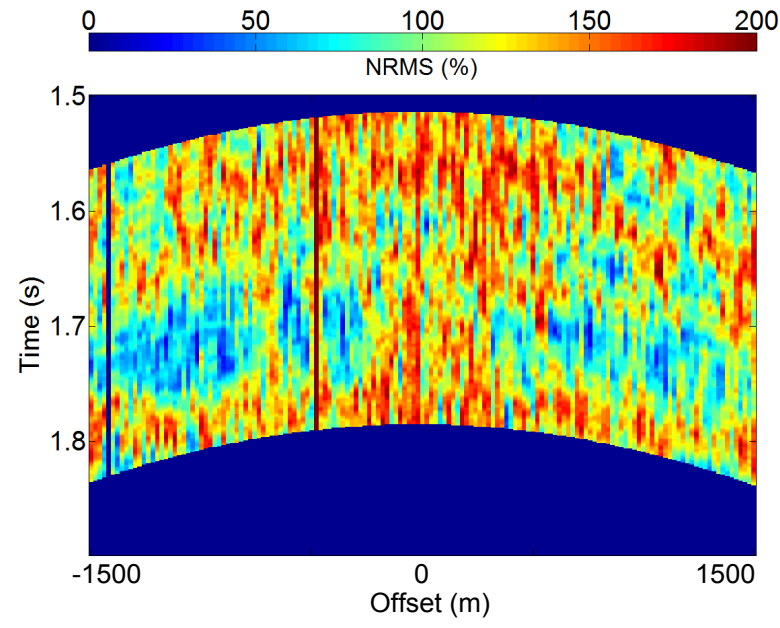

(d)

Figure 5.22: Simultaneous surface-consistent deconvolution for baseline (a), matched monitor (b), difference (c), and the NRMS error (d). A bandpass filter (4-8-90-100 Hz) is applied for display. Plots (b) and (c) have the same amplitude scaling as plot (a). 
related to the fold of each of the component. For example, when the fold is high, the computed correction is low and vice versa.

Figure 5.24 illustrates the results of applying the four-terms SCAC. The amplitude is balanced especially at the far offsets when compared to that shown in Figure 5.22. Despite this correction in amplitude, the NRMS error does not show significant improvement.

After SCAC, we picked the first break times and computed datum statics using a replacement velocity of $2500 \mathrm{~m} / \mathrm{s}$ and an elevation of $910 \mathrm{~m}$. Then we applied an initial velocity model based on the baseline survey to correct for normal-moveout (NMOC). A first pass of simultaneous surface-consistent residual statics is computed using a similar approach to that of SCAC in equation 5.3, except that $\mathbf{d}_{b}$ represents the baseline survey time-shifts and $\mathbf{d}_{m}$ represents the monitor survey time-shifts. $\mathbf{x}$ contains the fourcomponents surface-consistent static corrections (SCSC) to be determined. This is then followed by a careful pick of the final velocity, picked on the baseline survey only, then another pass of simultaneous SCSC. 


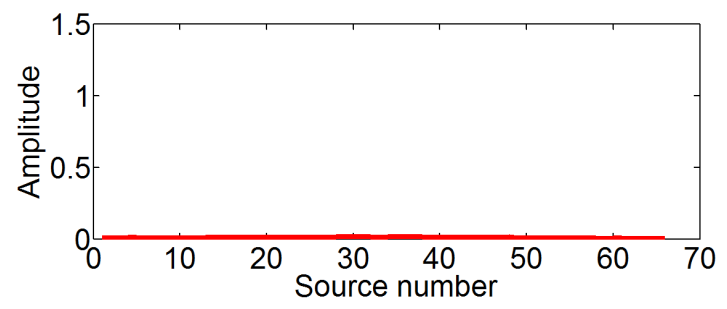

(a)

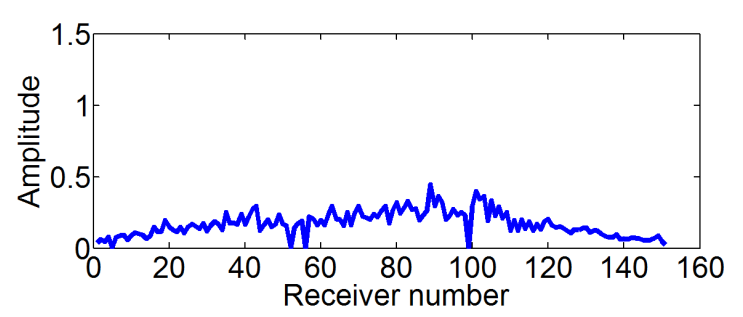

(c)

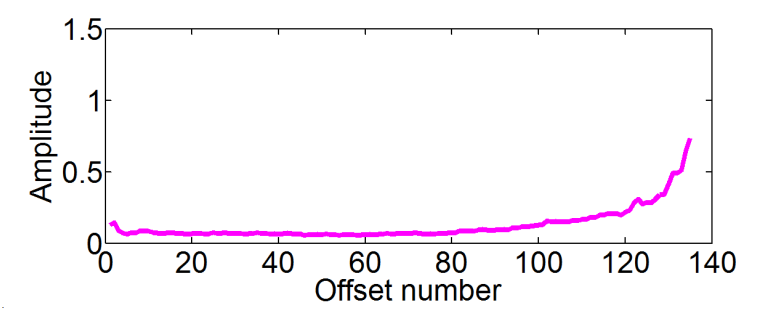

(e)

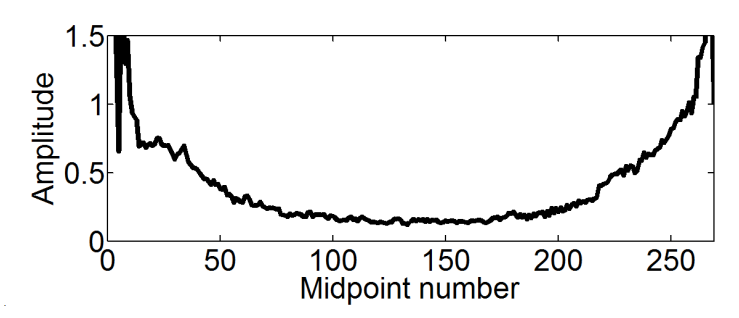

(g)

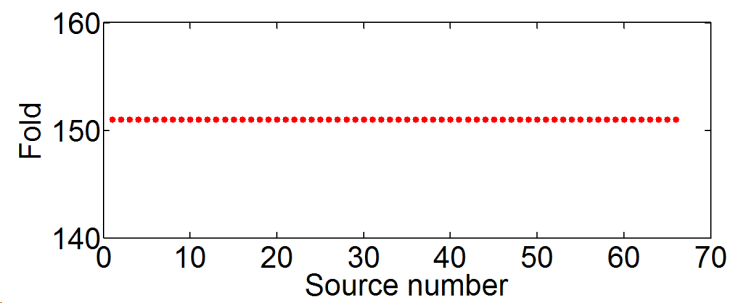

(b)

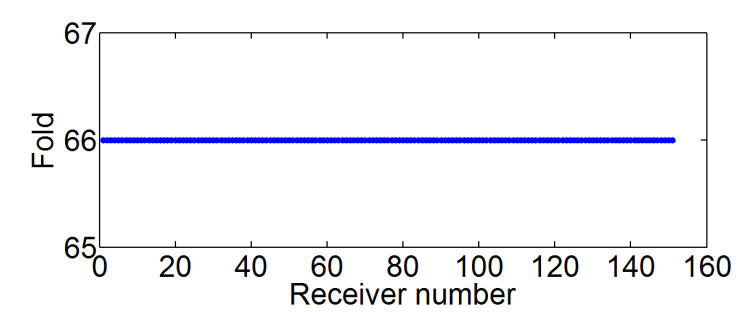

(d)

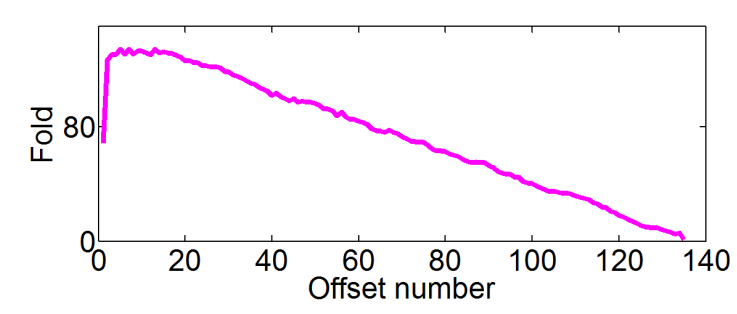

(f)

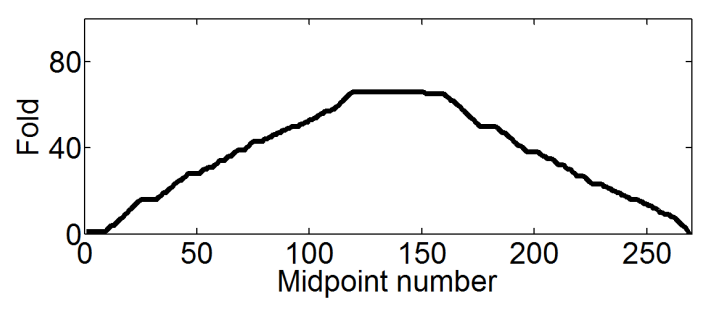

(h)

Figure 5.23: Simultaneous surface-consistent amplitude corrections (SCAC) and their corresponding fold. The panels show the SCAC for sources-term (a), the source fold (b), the SCAC for the receivers-term (c), the receiver fold (d), the SCAC for offsets-term (e), the offset fold (f), the SCAC for the midpoints $(\mathrm{g})$, and the midpoint fold $(\mathrm{h})$. 


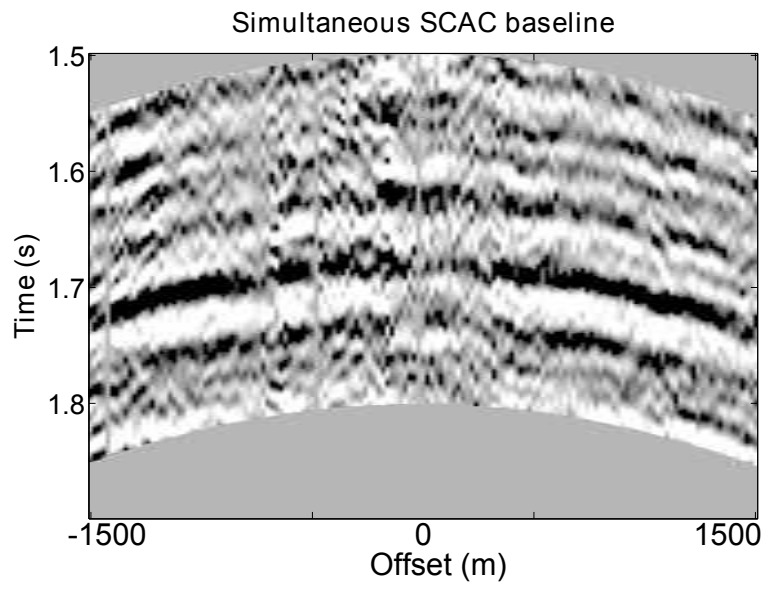

(a)

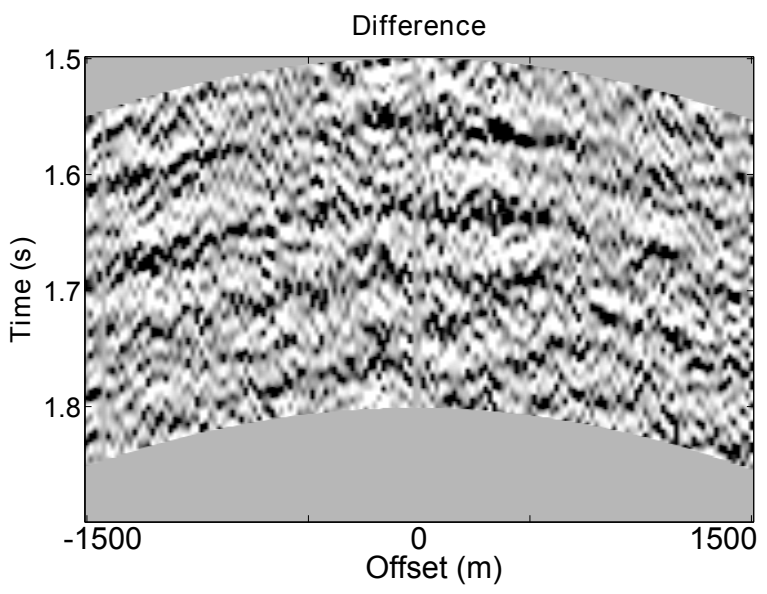

(c)

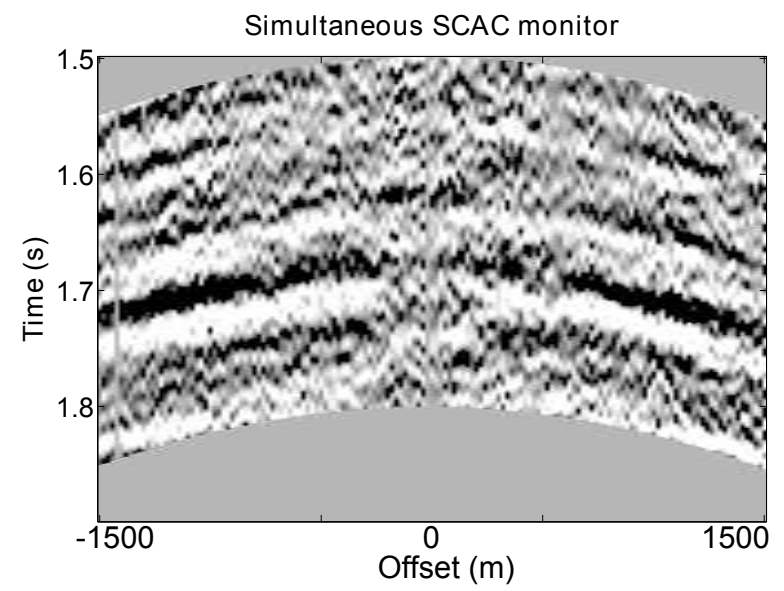

(b)

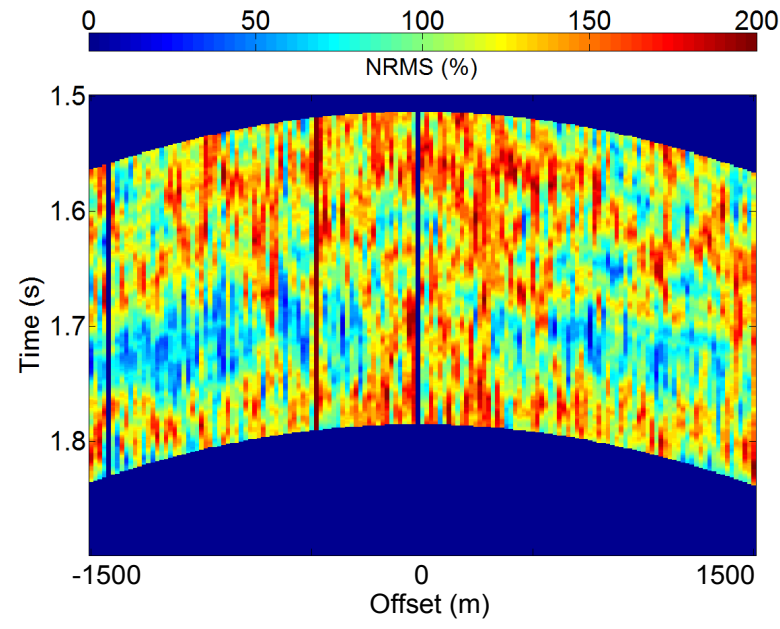

(d)

Figure 5.24: Simultaneous surface-consistent amplitude correction for baseline (a), matched monitor (b), difference (c), and the NRMS error (d). A bandpass filter $(8-12-55-70 \mathrm{~Hz})$ is applied for display. Plots (b) and (c) have the same amplitude scaling as plot (a). 


\subsection{Poststack Analysis of the processed data}

In this section, we will discuss the poststack results of both parallel and simultaneous processing. Table 5.4 shows the parallel processing where CMP stacking is followed by FX-deconvolution (Canales, 1984) for noise attenuation and Kirchhoff migration. The FX-deconvolution applies a Fourier transform to each trace of the stacked data, followed by a complex Wiener filter in space for each frequency, and then inverse transform back to the time domain (Canales, 1984). This method reduces the incoherent noise from the seismic data. In the simultaneous processing flow, the survey data are separated for CMP stacking, FX-deconvolution, and Kirchhoff migration. All stack displays in this section have bandpass filter of 8-12-55-70 Hz applied, and a mean scaling of $1000 \mathrm{~ms}$. Moreover, all plots have exactly the same amplitude clipping as that of the baseline stack. NRMS

plots will be used as a measure of repeatability plotted with the same scaling limits (0 to $200 \%$ ). Since poststack matching is common in time-lapse processing, we will be presenting the result of applying it to both parallel and simultaneous processing and we will include NRMS error analysis.

\subsubsection{Parallel processing}

Figure 5.25 shows the parallel processing result for the baseline survey, the monitor survey, and their difference. In the difference section, we observe residual higher amplitude events, especially between $1.2 \mathrm{~s}$ and $1.4 \mathrm{~s}$, in addition to differences below $1.4 \mathrm{~s}$ but at slightly lower amplitudes. Note the differences at the edges are due to loss of fold. Because we processed both lines independently, we do not expect a close match (both in amplitudes and time) due to the difference in random noise and the possibilities that the near-surface conditions were slightly different.

The computed NRMS error is shown in Figure 5.26. A histogram plot of the error shows that the mean error is $\sim 75 \%$. Note also that most of the red color relates to 
errors higher than $100 \%$ which might be indicative of the high amplitude noise trends near zero-offset observed in the prestack data which were not canceled by stacking. On the other hand, examining the error in the $300 \mathrm{~ms}$ window of analysis (light blue color), which is the window of the SCMF discussed earlier in 5.3.3, it averaged $\sim 42 \%$.

\subsubsection{Simultaneous processing}

We stacked the result of the simultaneous processing, shown in Section 5.4.2, independently. We also applied FX-deconvolution and Kirchhoff migration on the stacked data. Figure 5.27 displays the stack result of the baseline survey, the SCMF monitor, and their difference. The difference stack show much less coherent event when compared to Figure 5.25(c). The high amplitude residuals are patchy and can be attributed to: 1) low fold at the edges of the seismic line, and 2) incoherent noise that failed to stack out (particularly between $1 \mathrm{~s}$ and $1.3 \mathrm{~s}$ ). We noted earlier in Figure 5.23 that the offset and midpoint terms have variable fold which impacts their solution. When the fold is high, the solution is highly accurate, but when the fold is low, it negatively impacts the solution.

Figure 5.28 shows the NRMS error computed from the stacks of the simultaneous processing. The mean value of the NRMS error is down by about $25 \%$ compared to parallel processing. Although the reduced mean value does not seem that high after applying the SCMF and the simultaneous processing, we notice that many of the residuals with errors greater than $75 \%$ have been reduced significantly. The occurrences of yellow and red colors, indicating errors higher than 75\%, are greater in Figure 5.26(a) compared to Figure 5.28(a).

The mean NRMS error inside the SCMF window of analysis is $\sim 27 \%$. The low NRMS error indicates a good repeatability between the two time-lapse surveys after applying the SCMF and the simultaneous processing flow. 


\subsubsection{Poststack matching}

Now that we have examined the results of independent and simultaneous processing, we analyze the stacks after poststack matching. We applied a match filtering technique that is a least-squares algorithm that is commonly used to minimize differences between two traces. The difference can be related to amplitude, or time or both. The match filter that we use here minimizes squared error (L2 Norm) between two datasets using a finite length filter. The time window of the filter is from $1.0 \mathrm{~s}$ to 2.0 with a filter length equal to the trace length, i.e. $1.0 \mathrm{~s}$. We also used a $5 \%$ additive noise factor to stabilize the inversion and applied a Hanning (cosine) taper to the input traces.

Figure 5.29 shows the parallel processing stacks for the baseline, the monitor after applying match filter, and their difference. An average $-2.0 \mathrm{~ms}$ time shift was computed from crosscorrelating traces in the monitor stack to the same ones in the baseline stack. After shifting the monitor stack to match the baseline, a least-squares match filter to adjust amplitudes and phase was computed and applied. The NRMS error was computed and is shown in Figure 5.30. The histogram plot of NRMS error shows a mean value close to $25 \%$ and the mean NRMS error in the $300 \mathrm{~ms}$ window of analysis (light blue color) is about $12.7 \%$.

Comparing the results above to the simultaneous stacks in Figure 5.31 after computing and applying poststack match filters, they appear to be very similar. There was no timeshift required in the case of the SCMF and simultaneously processed monitor stack when crosscorrelated with the baseline. This indicate that the variations observed in Figure 5.27(c) were mostly amplitude differences. The NRMS error is shown in Figure 5.32 and is very similar to that of the parallel processing result after poststack match filters. The mean NRMS error in the window of analysis (light blue color) is $12.8 \%$, similar to that reported in the parallel processing after poststack match fitlers. 


\subsection{Summary}

In this chapter, we examined a $2 \mathrm{D}$ time-lapse data set from a pilot $\mathrm{CO}_{2}$ injection site in Alberta, Canada. There were many challenges in this data set, as is the case with many land seismic datasets, including coherent and incoherent noise. Attenuating the coherent noise is not a challenge providing that these noise have dips distinguishable from primary events. In addition to noise, near surface seasonal variations can have nonrepeatable effects on the downgoing and upgoing wavefields. The near surface acts as a filter to the passing wavefields and affects their traveltimes and amplitudes. The last challenge, which is specific to this data set, is difficulty in imaging the target zone either before or after the gas injection. The imaging problem is due to a thin reservoir with indistinct acoustic impedance contrast.

We attenuated most of the linear noises except those with high energy and concentrated in the near offsets of the shot records. The difficulty with these noises is that their dips were similar to those of primary reflections. We had to change our strategy in processing those data from using a match filter design window above the reservoir to a different one below the reservoir. We computed the surface-consistent match filter over a $300 \mathrm{~ms}$ window (light blue color). The four-component SCMF were computed and applied to the monitor survey to match it to the baseline. The prestack result is encouraging but due to high noise level, other processing such as deconvolution, amplitude corrections and CMP stacking incrementally improved the repeatability of both data sets.

The data set was processed using two processing schemes:

1. parallel processing scheme, and

2. a modified simultaneous processing scheme where both data sets were merged for all surface-consistent process. 
The modified simultaneous processing scheme benefited from the SCMF where the merged surveys share a common source, a common receiver, a common offset, and a common midpoint solution for every co-located term in all surface-consistent process. This modification is an improvement over the existing time-lapse simultaneous processing scheme where the merged data set share a common midpoint operator and in some cases a common offset operator.

The simultaneous processing flow after applying the SCMF improved the repeatability of the monitoring seismic data. The independent processing scheme was unable to overcome the residual in amplitude and time between the two surveys. These differences were easy to observe after CMP stacking. Other than the high amplitude random noise that are still persistent in the final stack, the simultaneous processing result is clearly better than the parallel processing stack section.

Finally, we applied a poststack match filter, which included a time-shift and an amplitude correction to the parallel processing result. The error between both data sets reduced significantly averaging about $12 \%$. Similarly, a poststack match filter is applied to the simultaneous processing result but only for amplitude correction since time shift was not required. The final result was similar to that obtained from the parallel processing after the poststack match filter. 


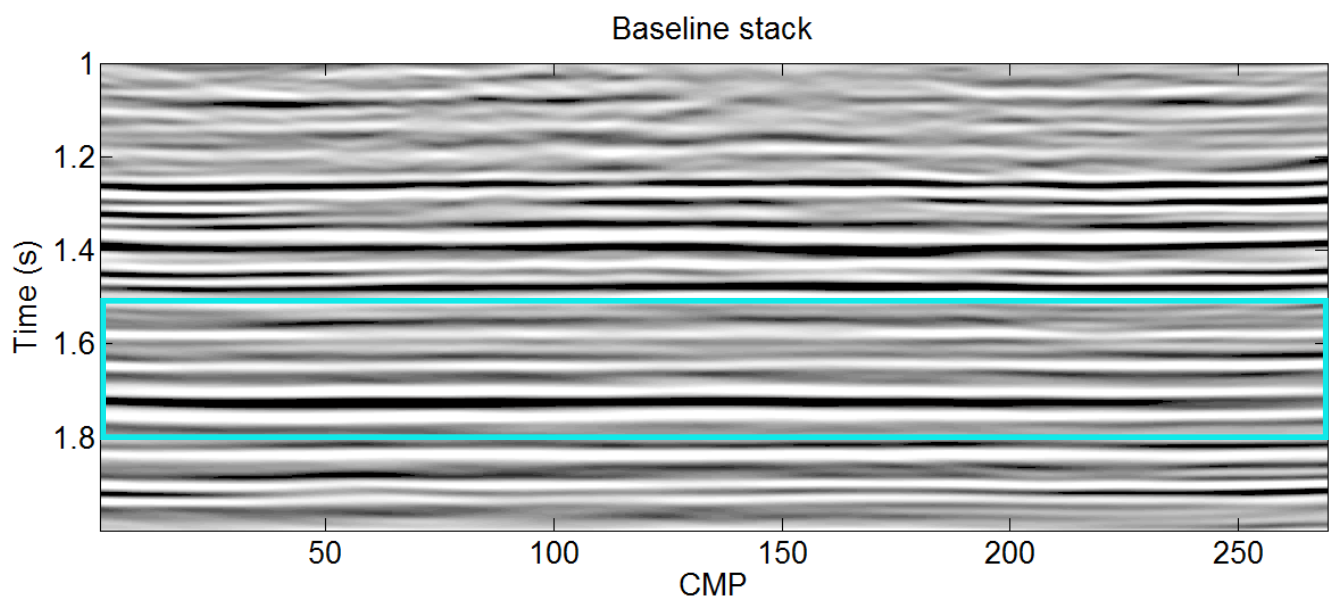

(a)

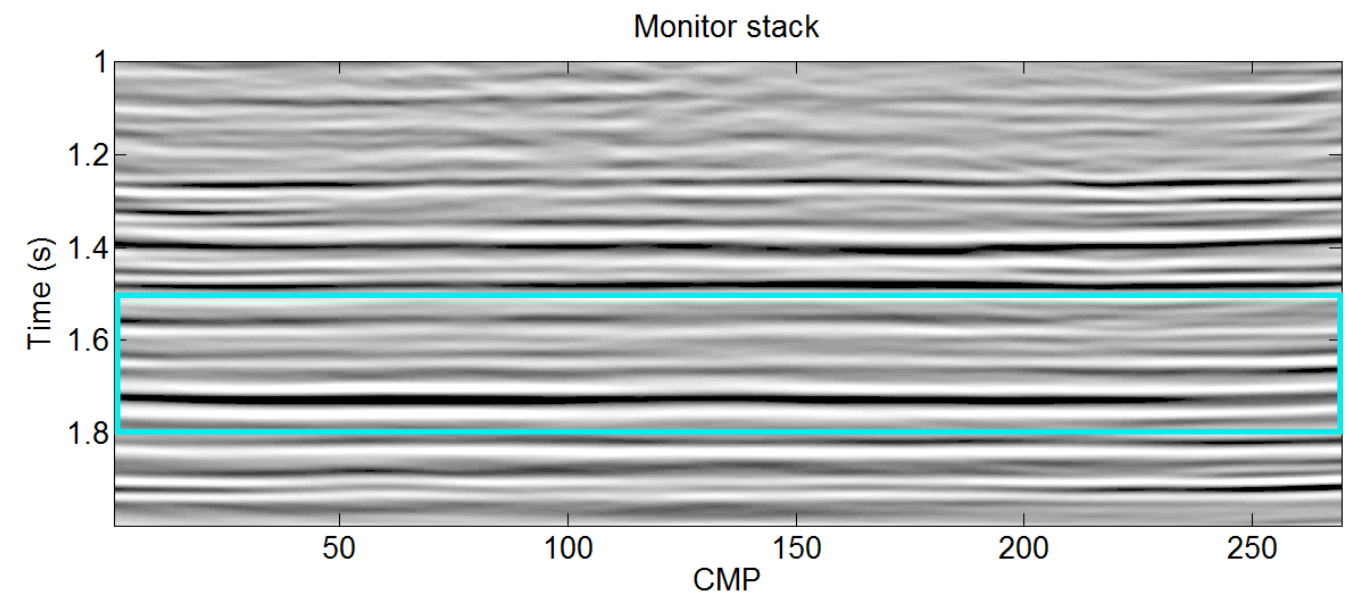

(b)

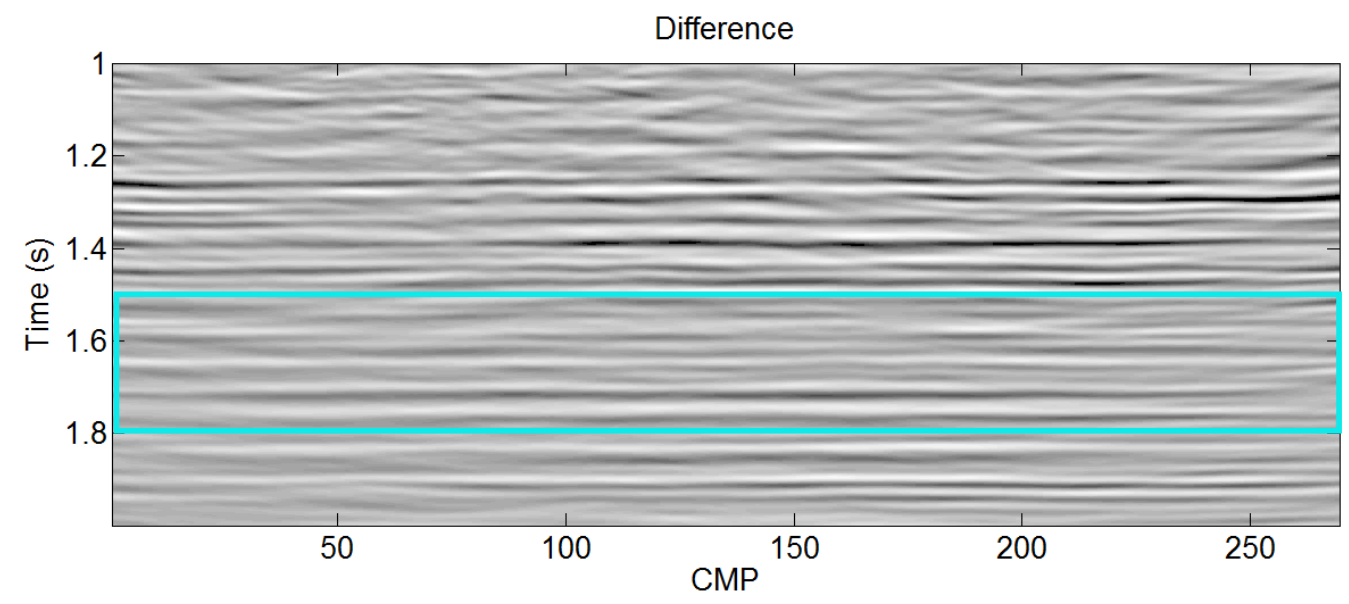

(c)

Figure 5.25: Parallel processing stacking result after poststack Kirchhoff migration, FX-deconvolution, and for display has a bandpass filter (8-12-55-70) and $1000 \mathrm{~ms}$ window scaling. (a) is the baseline stack, (b) is the monitor stack, and (c) is the difference. 


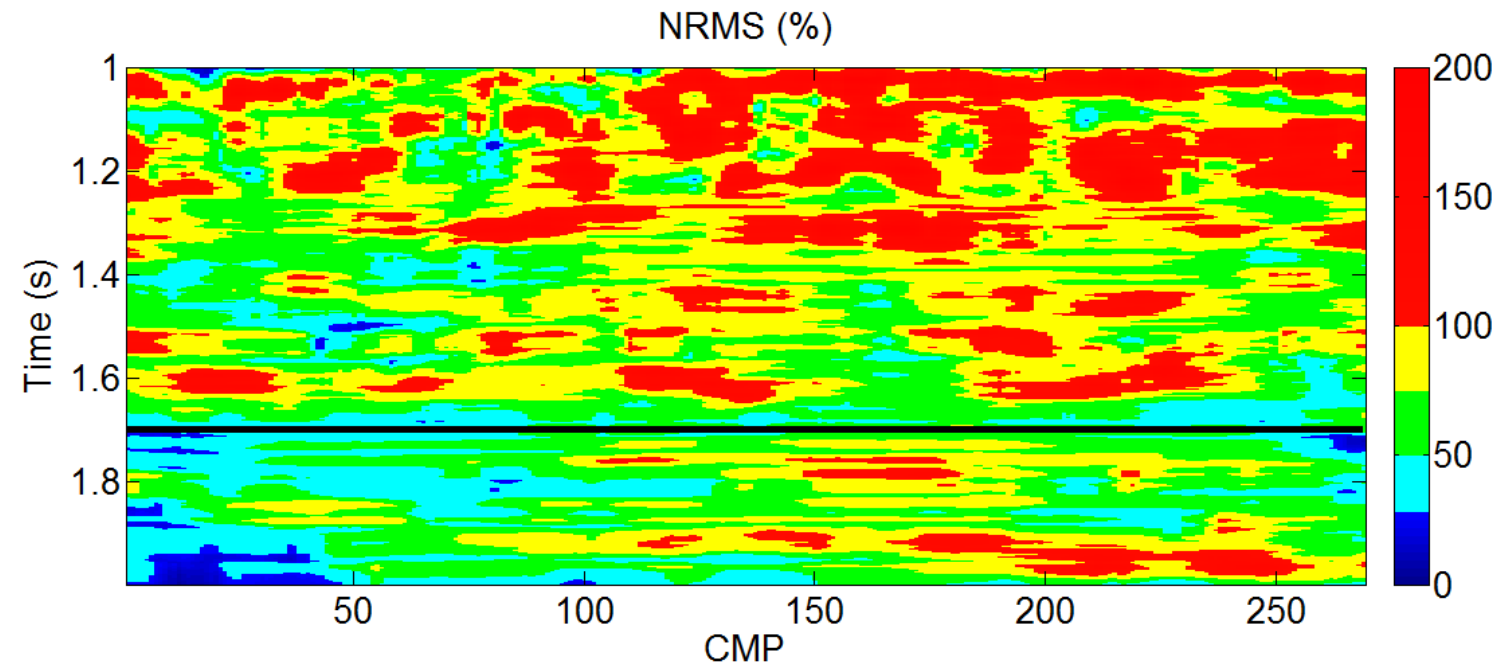

(a)

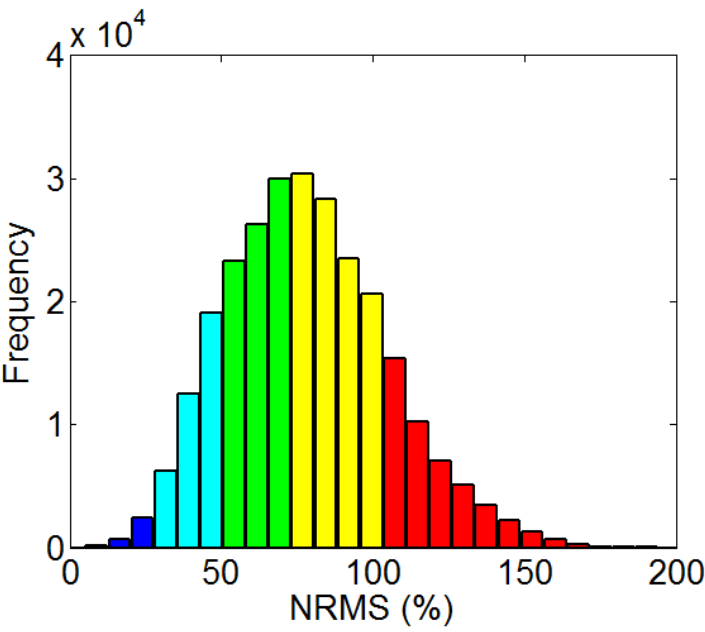

(b)

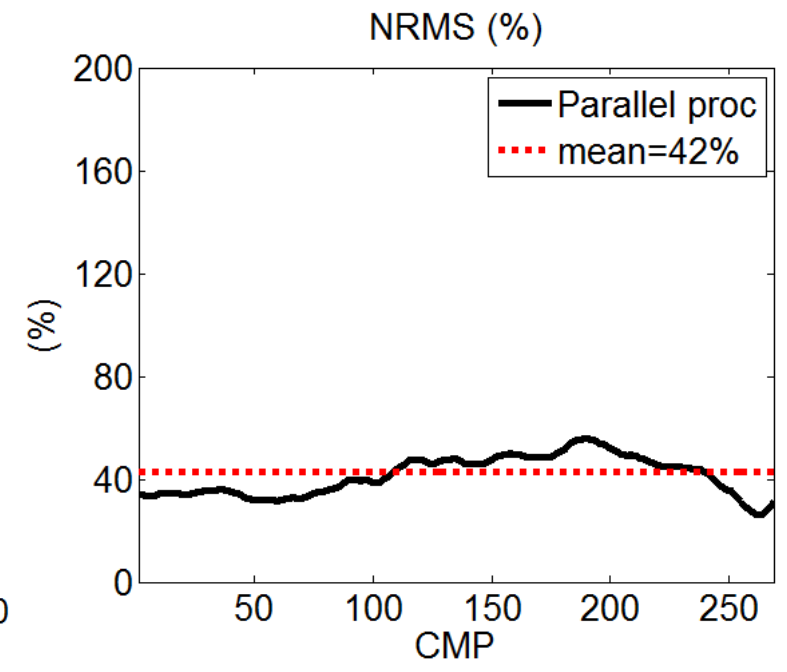

(c)

Figure 5.26: (a) shows the NRMS error of the stacks in Figure 5.25. (b) shows a histogram of the error versus frequency and (c) shows an average of the NRMS error computed from the window of analysis (light blue box) in Figure 5.25. 
Baseline stack

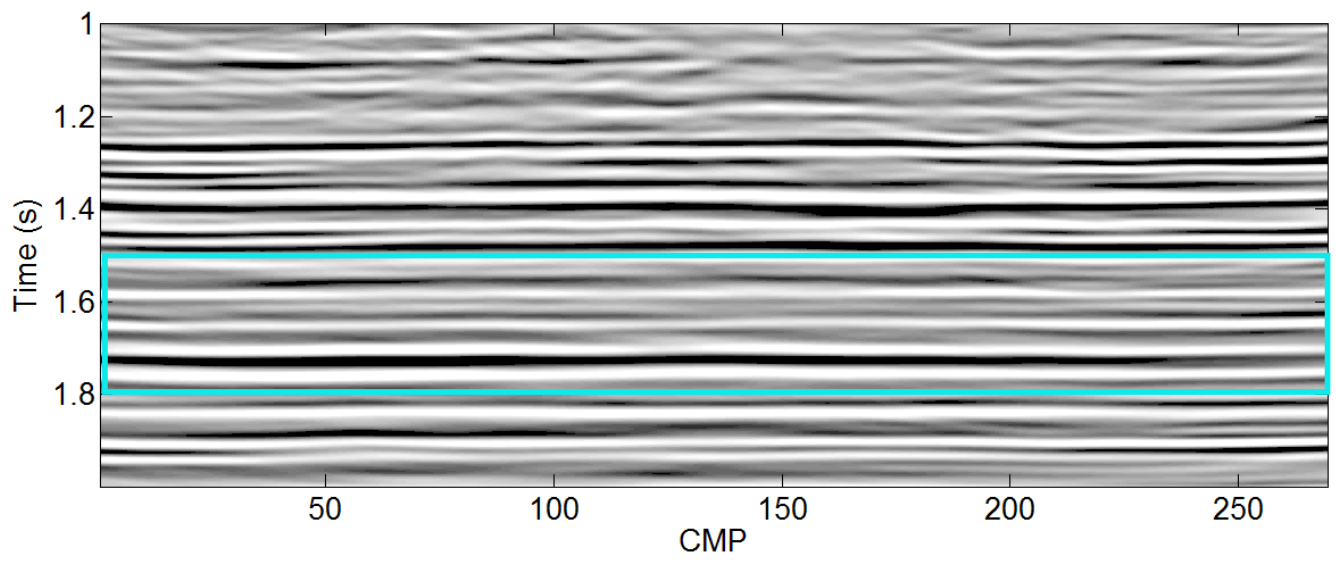

(a)

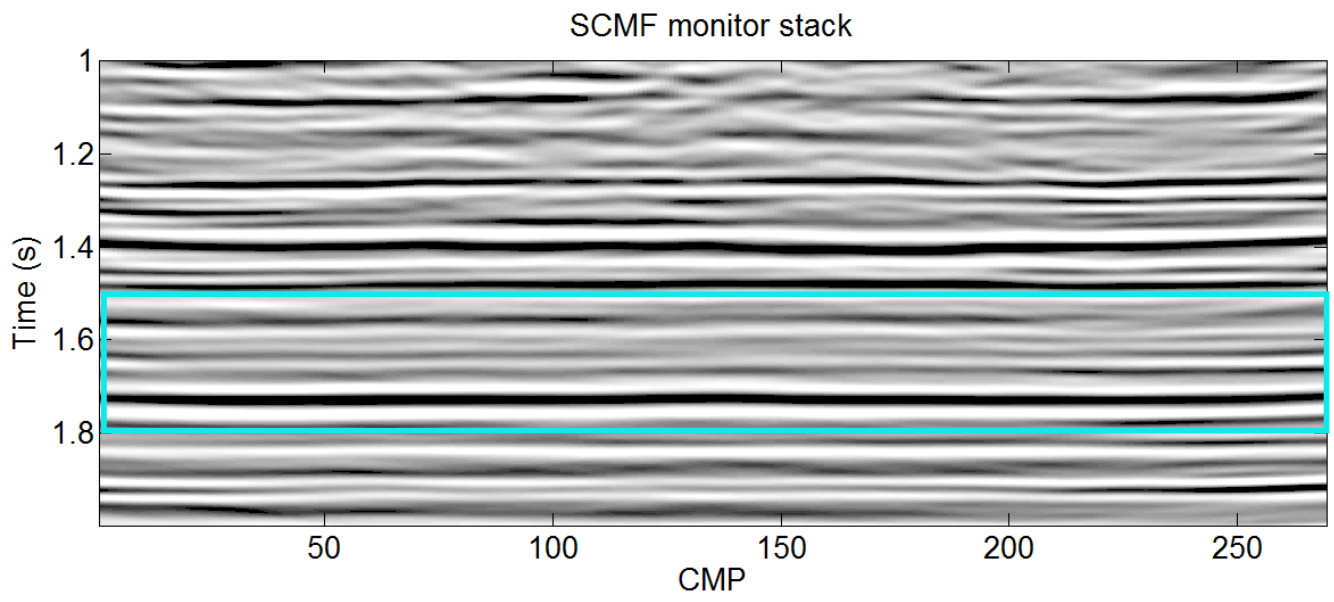

(b)

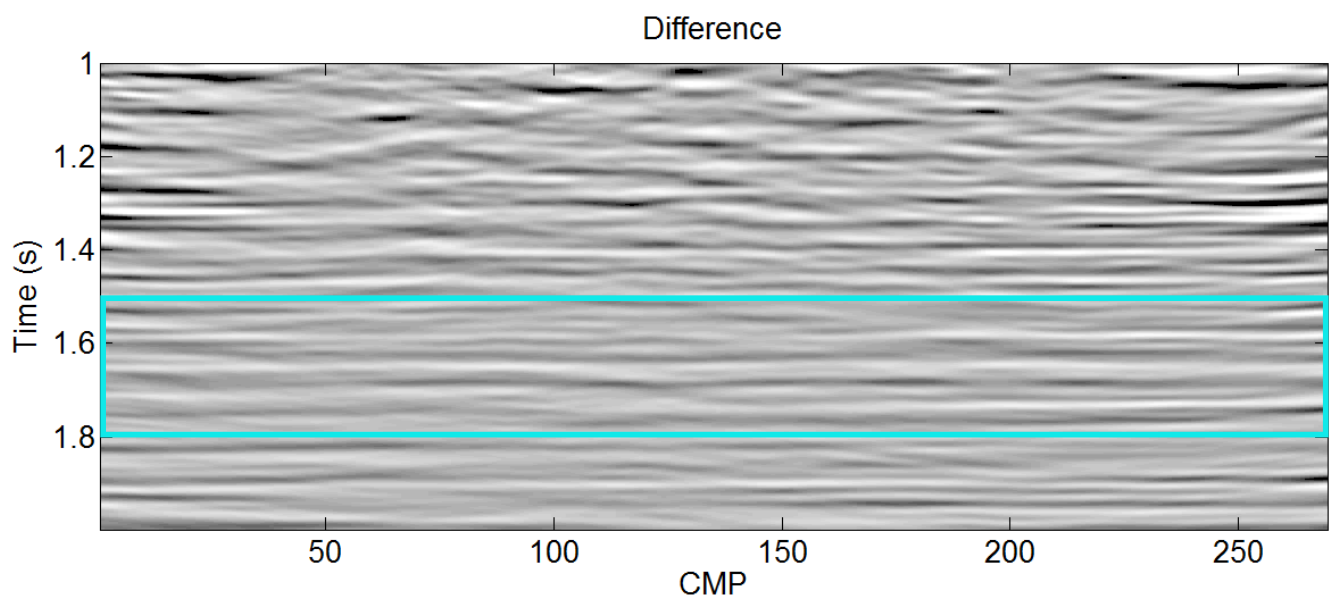

(c)

Figure 5.27: Simultaneous processing stacking result after poststack Kirchhoff migration, FX-deconvolution, and for display has a bandpass filter (8-12-55-70) and $1000 \mathrm{~ms}$ window scaling. (a) is the baseline stack, (b) is the SCMF monitor stack, and (c) is the difference. 


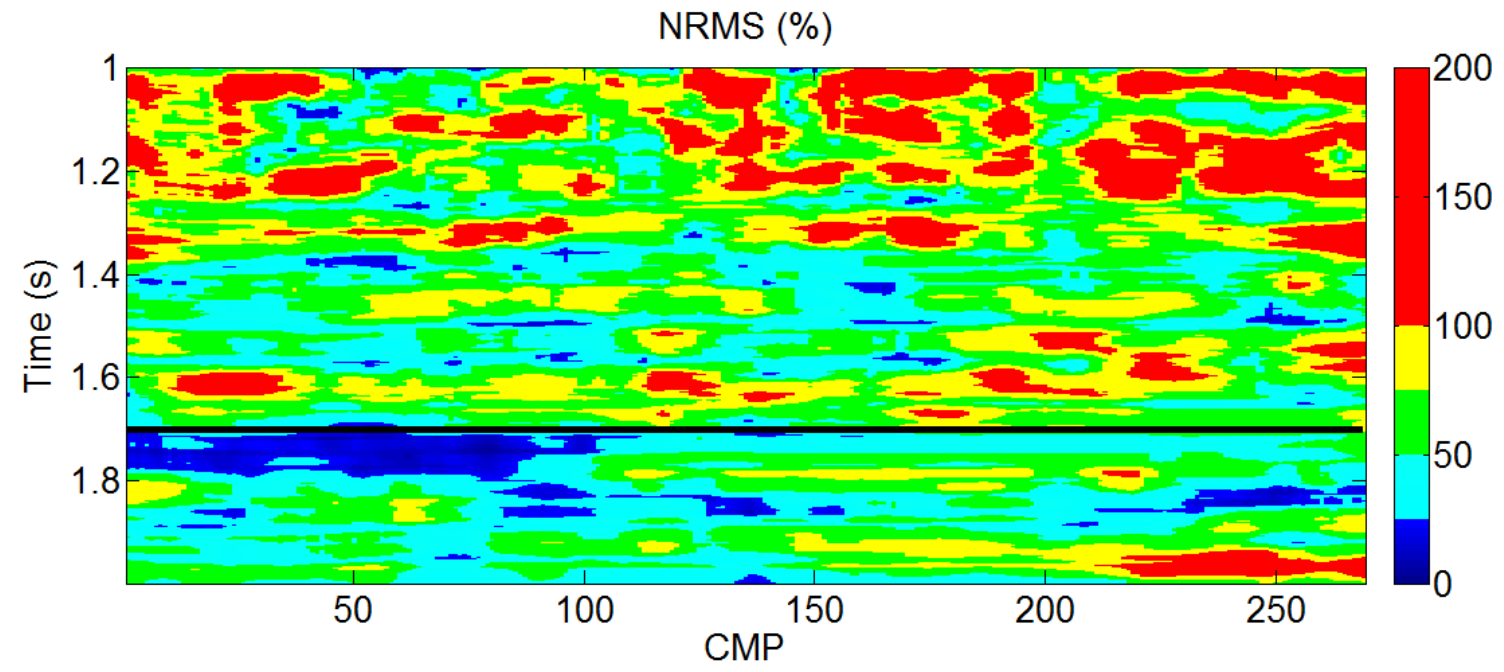

(a)

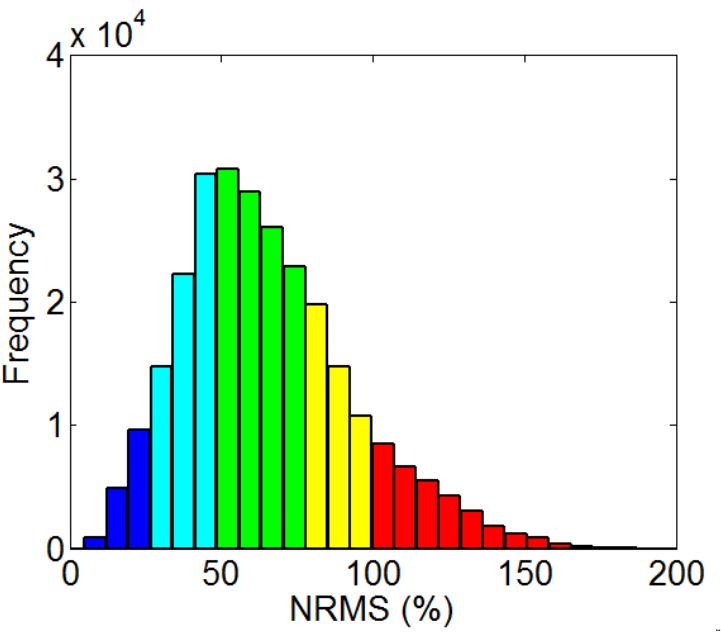

(b)

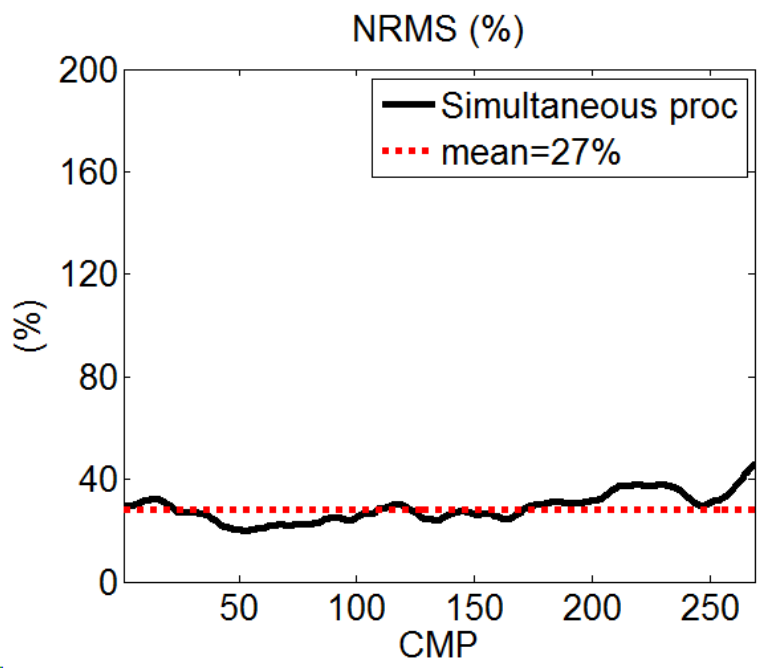

(c)

Figure 5.28: (a) shows the NRMS error of the stacks in Figure 5.27. (b) shows a histogram of the error versus frequency and (c) shows an average of the NRMS error computed from the window of analysis (light blue box) in Figure 5.27. 
Baseline stack

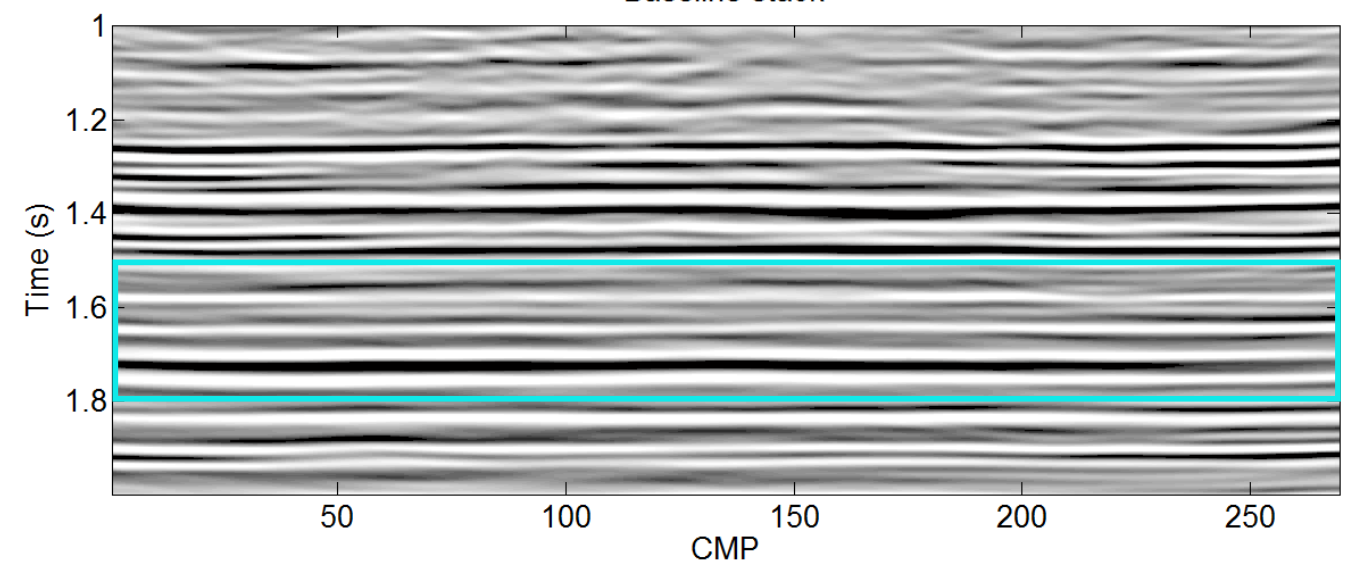

(a)

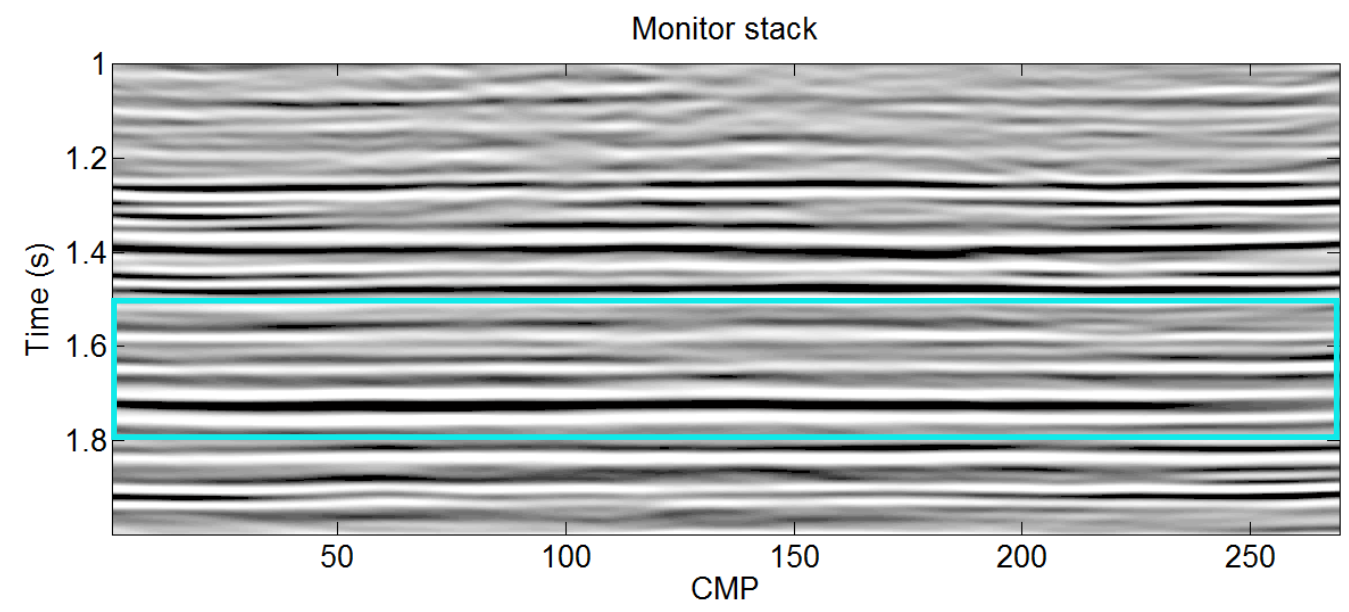

(b)

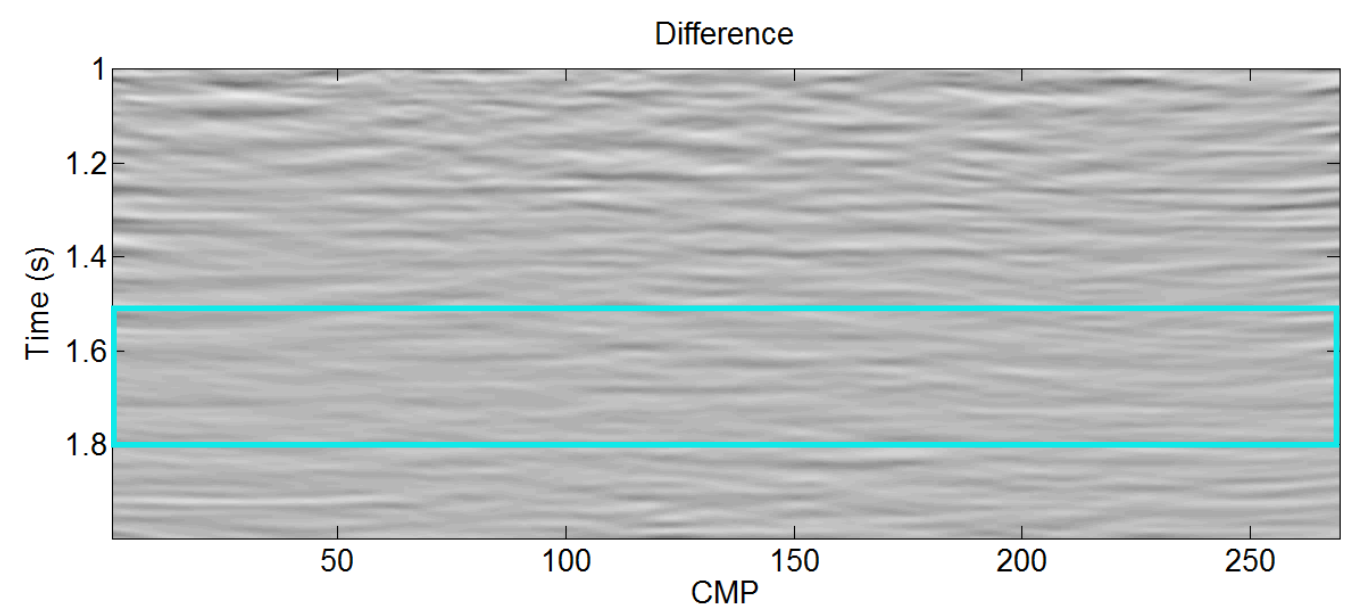

(c)

Figure 5.29: Parallel processing stacking result shown in Figure 5.25 after poststack matching of amplitudes and time-shifts. (a) is the baseline stack, (b) is the monitor stack after matching, and (c) is their difference. 


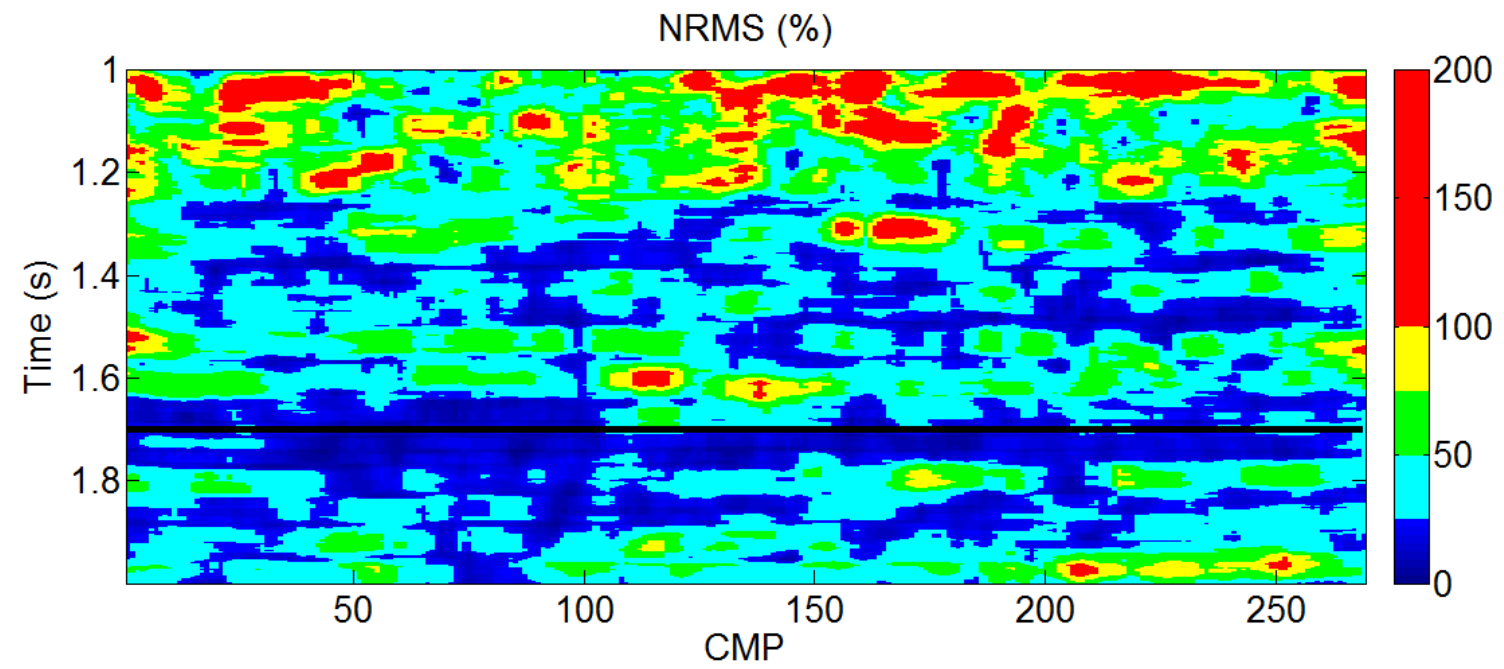

(a)

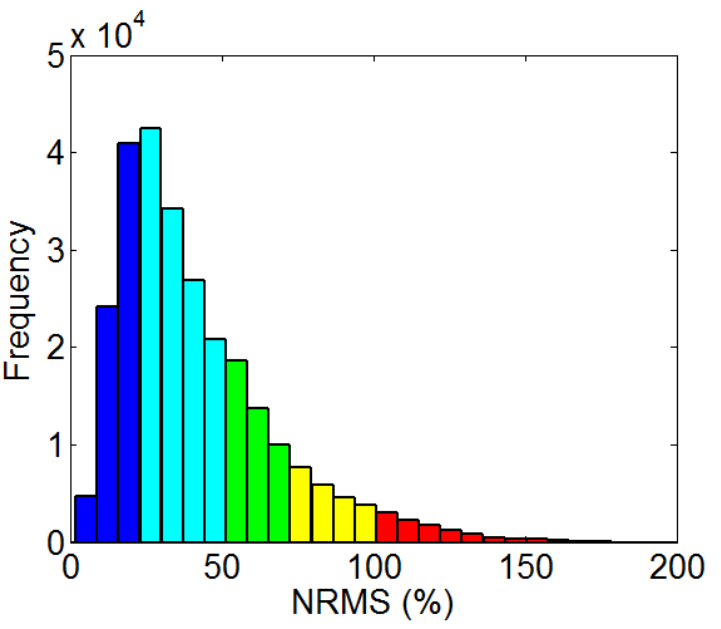

(b)

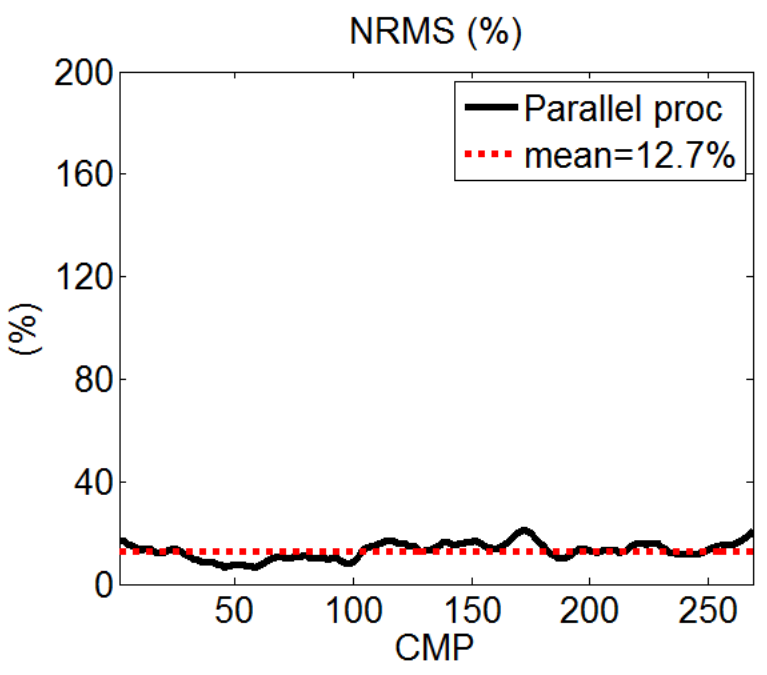

(c)

Figure 5.30: (a) shows the NRMS error of the stacks in Figure 5.25. (b) shows a histogram of the error versus frequency and (c) shows an average of the NRMS error computed from the window of analysis (light blue box) in Figure 5.29. 


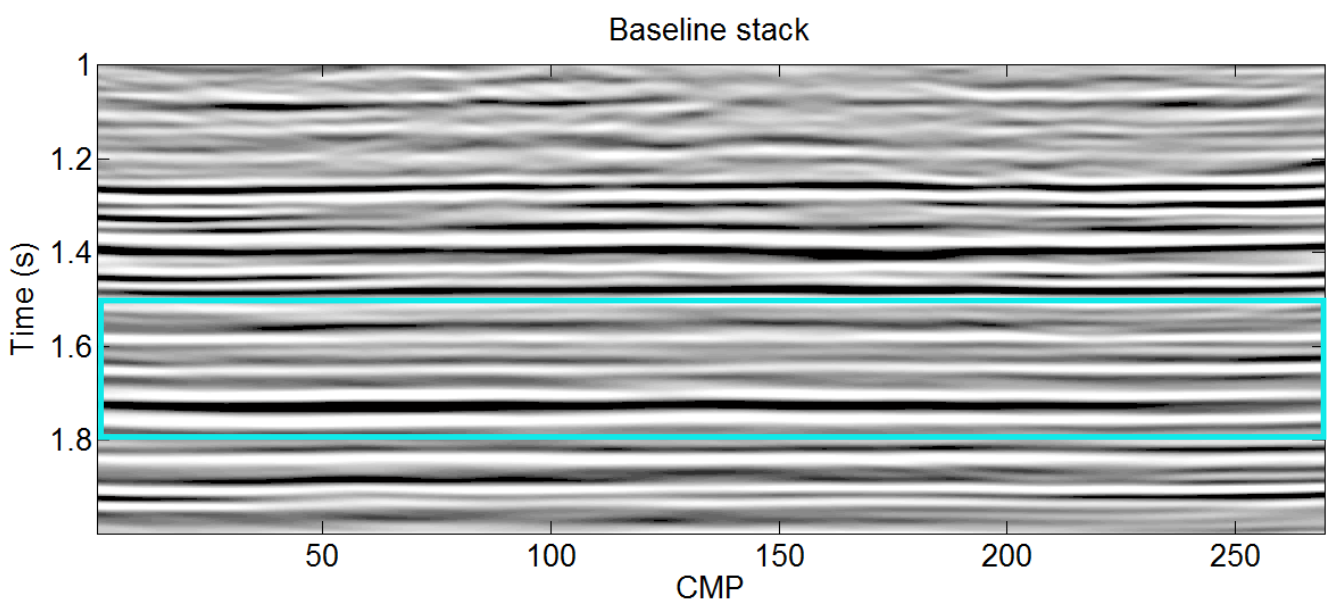

(a)

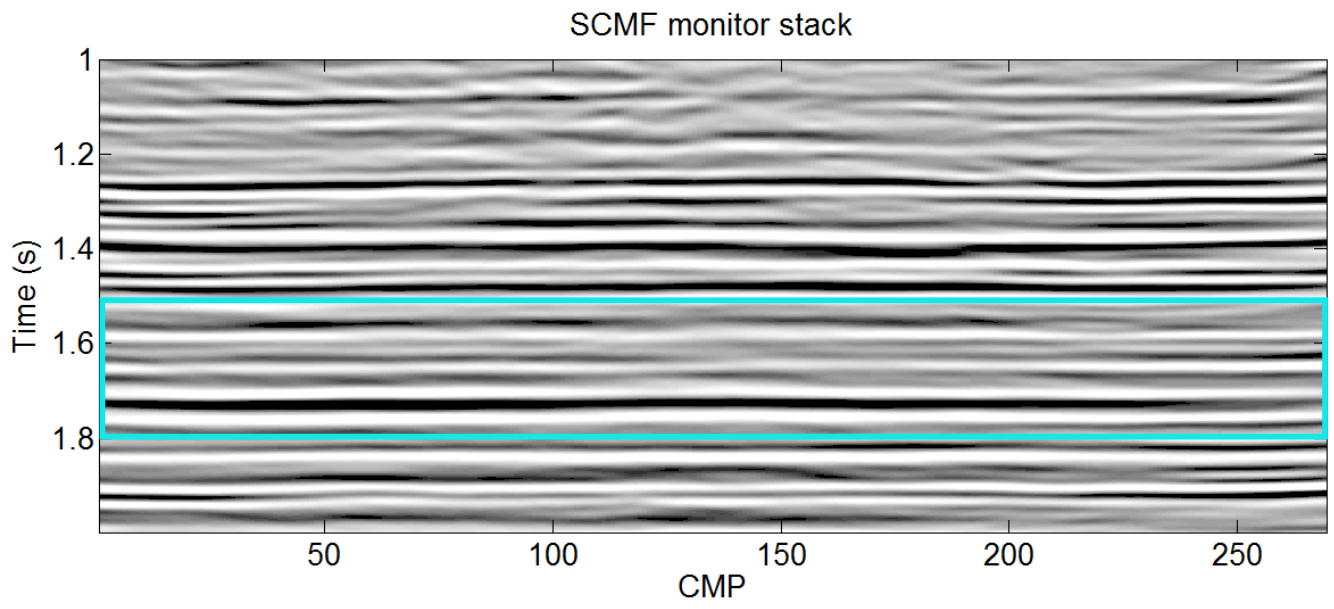

(b)

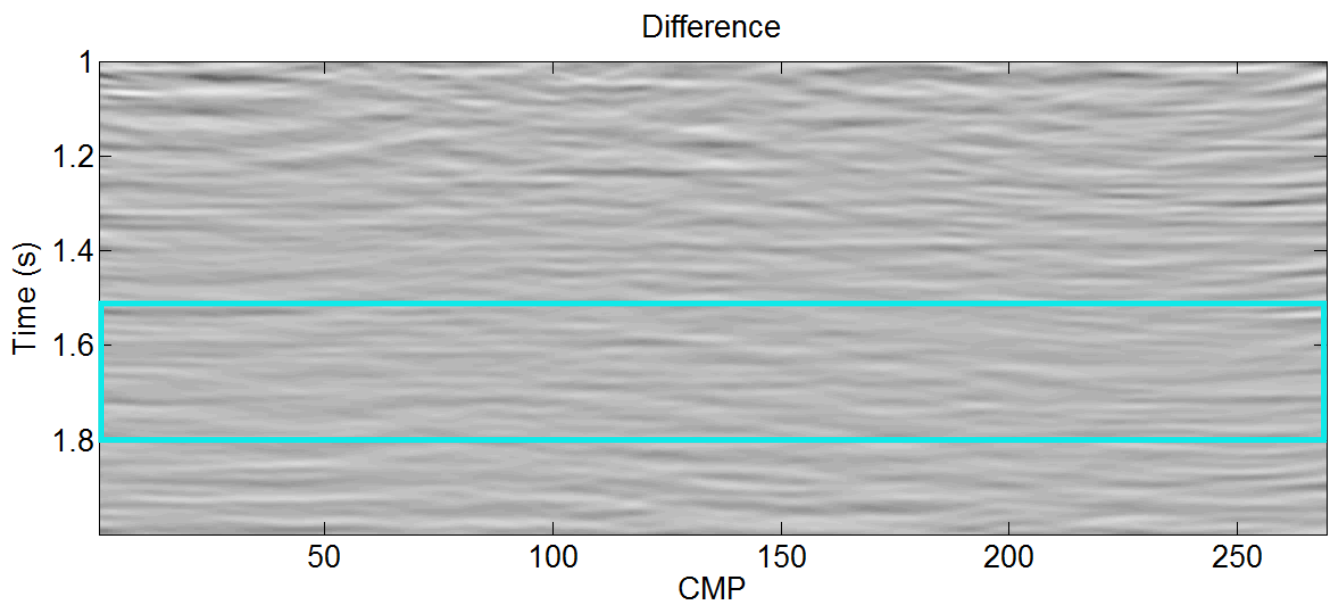

(c)

Figure 5.31: Simultaneous processing stacking result shown in Figure 5.27 after poststack matching of amplitudes and time-shifts. (a) is the baseline stack, (b) is the monitor stack after matching, and (c) is their difference. 


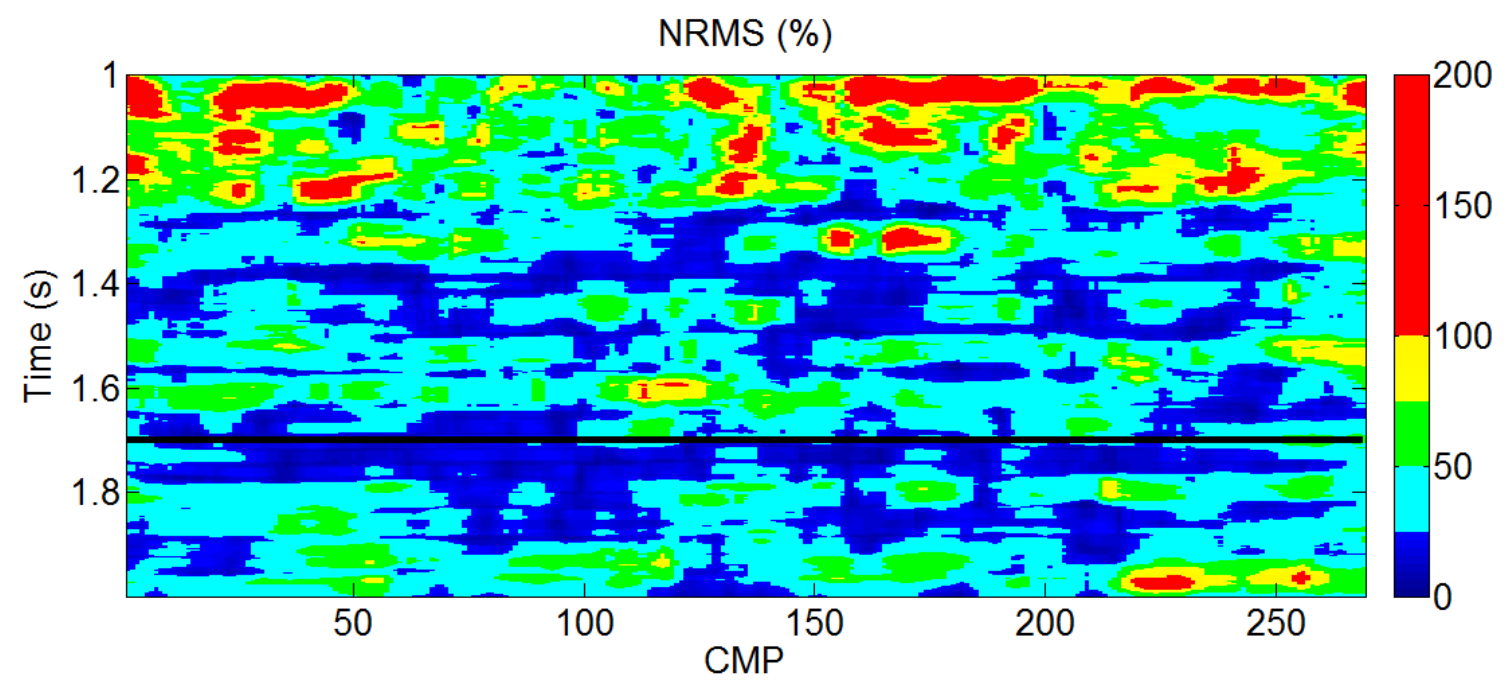

(a)

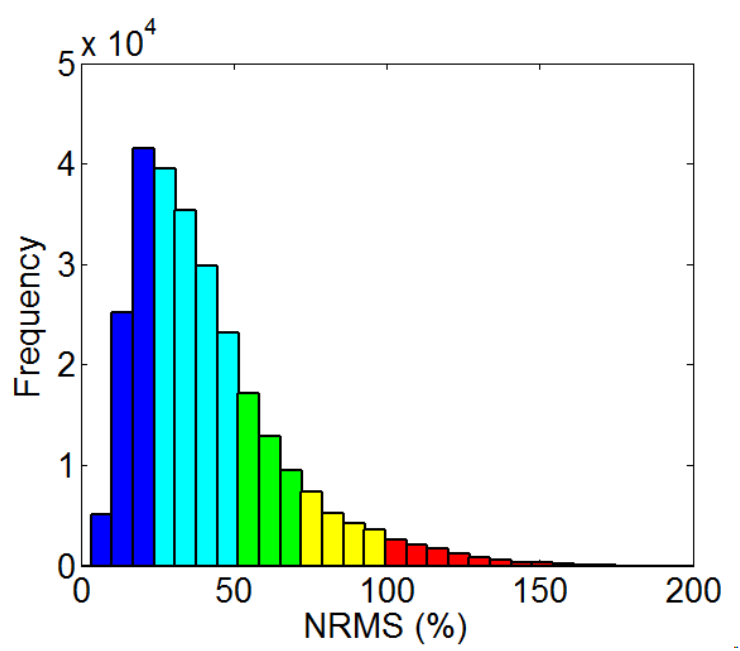

(b)

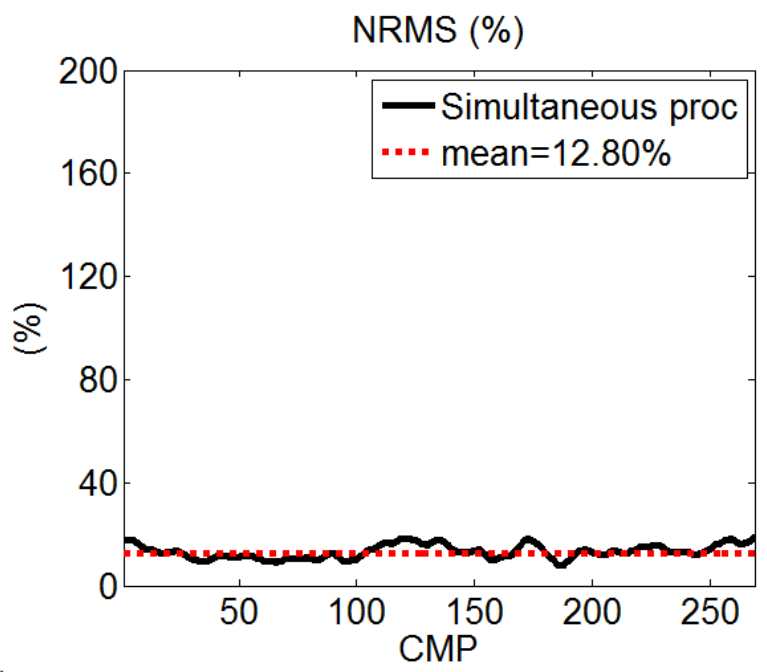

(c)

Figure 5.32: (a) shows the NRMS error of the stacks in Figure 5.25. (b) shows a histogram of the error versus frequency and (c) shows an average of the NRMS error computed from the window of analysis (light blue box) in Figure 5.31. 


\section{Chapter 6}

\section{Conclusions}

We have introduced new techniques that overcome some important issues in current timelapse processing schemes. We have demonstrated with difficult examples that the new algorithm and the modifications added to the simultaneous processing workflow provided good-quality time-lapse result from which we can deduce reliable information about the changes in the subsurface.

In chapter 2, we presented a review of the theory of the surface-consistent model, the model's basic assumptions, its basic form, and some of its applications in seismic data processing. We have also touched on some of the methods used to solve the surfaceconsistent equations for the statics problem, which is quite similar to the equation of the SCMF. The accuracy of the surface-consistent model was discussed and some of the factors that impact its result was briefly mentioned.

In chapter 3, we presented a new filter algorithm, which we termed surface-consistent matching filters (SCMF), that can be used to match one data set to another in a timelapse experiment. The new algorithm is an extension of the well known surface-consistent deconvolution where the data required are the trace-by-trace spectral ratios of two data sets instead of the spectrum of a single one. We have demonstrated that computing the spectral ratios in a stable manner is possible by Fourier transforming the trace-sequential time-domain least-squares matching filters. A subsequent least-squares solution then factors the trace-sequential matching filters into four surface-consistent operators: source, receiver, offset, and midpoint. The output of the algorithm is a set of surface-consistent convolutional filters with associated surface-consistent time shifts. These filters and time shifts will be used to match the monitor survey to the baseline survey in the least-squares 
sense.

In chapter 4, using a synthetic seismic model, we applied the four-term surfaceconsistent matching filters to the monitor survey to match it to the baseline survey over a temporal window above the reservoir. The prestack nonrepeatable difference between the matched monitor survey and the baseline survey was quite small compared to the difference before applying the SCMF. The simulated nonrepeatable effects are quite complex and included reflection amplitude variations, wavelet bandwidth and phase differences, and large static shifts averaging $26 \mathrm{~ms}$. We have demonstrated that on single trace gathers, trace-sequential matching filters could reduce the differences between timelapse surveys when compared to surface-consistent matching filters. However, after two iterations of simultaneous surface-consistent static correction and stacking, the tracesequential matching exhibits high NRMS (35\%) while the surface-consistent matching filters improve significantly (NRMS of $15.7 \%$ ). We have also shown that applying the four-component surface-consistent matching filters yielded the lowest NRMS difference $(15.7 \%)$ compared to applying only two-term or even three-term surface-consistent matching filters. Migration shows slight improvement with a mean NRMS of about $13.6 \%$.

Another application of the new algorithm on a field data set was presented in chapter 5. We compared the result of two processing schemes:

1. parallel processing scheme, and

2. a modified simultaneous processing scheme where both data sets were merged for all surface-consistent process.

The modified simultaneous processing scheme benefited from the SCMF where the merged surveys shared a common source, a common receiver, a common offset, and a common midpoint solution for every co-located term in all surface-consistent process. This modification is an improvement over the existing time-lapse simultaneous processing scheme 
where the merged data set share a common midpoint operator and in some cases a common offset operator. The independent processing scheme was unable to overcome the difference in amplitude and time between the two surveys. A poststack match filter which included a time-shift then followed by an amplitude correction to the parallel processing result reduced the error significantly to about $12 \%$. Similarly, a poststack match filter applied to the simultaneously processed stacks but only included amplitude correction since time shift was not required and the reduced error was about $12 \%$ too. 


\section{Bibliography}

Alshuhail, A. A. (2011). $\mathrm{CO}_{2}$ sequestration site characterization and time-lapse monitoring using reflection seismic methods. PhD Thesis, University of Calgary.

Alshuhail, A. A., Lawton, D. C., and Chabot, L. (2008). Pembina cardium $\mathrm{CO}_{2}$ monitoring project: time-lapse seismic analysis. CREWES report, (20):1-18.

Anstey, N. A. (1964). Correlation techniques: A review. Geophys. Prosp., (12):355-382.

Arts, R., Chadwick, A., Eiken, O., Thibeau, S., and Nooner, S. (2008). Ten years' experience of monitoring $\mathrm{CO}_{2}$ injection in the utsira sand at sleipner, offshore norway. First Break, (26):65-72.

Arts, R., Eiken, O., Chadwick, A., Zweigel, P., Meer, L., and Zinszner, B. (2002). Results and experiences from the first industrial-scale underground $\mathrm{CO}_{2}$ sequestration case (Sleipner Field, North Sea). IEA 6th international conference.

Aster, R. C., Borchers, B., and Thurber, C. H. (2005). Parameter estimation and inverse problem.

Bachu, S. and Bennion, B. (2008). Effects of the in-situ conditions on relative permeability characteristics of $\mathrm{CO}_{2}$-brine system. Environ Geol, (54):1707-1722.

Bakulin, A., Burnstad, R., Jervis, M., and Kelamis, P. (2012). Evaluating permanent seismic monitoring with shallow burried sensors in a desert environment. SEG Exp. Abst.

Barkved, O. I. (2012). Seismic Surveillance for Reservoir Delivery: From a Practitioner's Point of View. EAGE Education Tour Series 6. 
Beasley, C. J., Chambers, R. E., Workman, R. L., Craft, K. L., and Meister, L. J. (1997). Repeatability of 3-d ocean-bottom cable seismic surveys. TLE, (16):1281-1285.

Calvert, R. (2005). Insights and methods for 4d reservoir monitoring and characterization. SEG/EAGE DISC course.

Cambois, G. and Stoffa, P. L. (1992). Surface-consistent deconvolution in the log/fourier domain. Geophysics, (57):823-840.

Cambois, G. and Stoffa, P. L. (1993). Surface-consistent phase decomposition in the $\log /$ fourier domain. Geophysics, (58):1099-111.

Campbell, S., Ricketts, T., Davies, D. M., Slater, C. P., Brain, J., Stammeijer, J., and Evans, A. C. (2005). Improved 4D seismic repeatability - a West of Shetlands towed streamer acquisition case history. SEG Exp. Abst., (24):2394.

Canales, L. L. (1984). Random Noise Reduction. SEG Exp. Abst.

Cary, P. W. and Lorentz, G. A. (1993). Four-component surface consistent deconvolution. Geophysics, (58):383-392.

Chadwick, A., Noya, D., Artsb, R., and Eikenc, O. (2009). Latest time-lapse seismic data from Sleipner yeild new insights into $\mathrm{CO}_{2}$ plume development. Energy Procedia.

Chen, F. (2006). Interpretation of time-lapse surface seismic data at a $\mathrm{CO}_{2}$ injection site, violet grove, alberta. MSc. Thesis, University of Calgary.

Claerbout, J. F. (1976). Fundamentals of Geophysical Data Processing With Applications to Petroleum Prospecting.

Claerbout, J. F. (1986). Simultaneous pre-normal and post-normal moveout deconvolution. Geophysics, (51):1341-1354. 
Clochard, V., Delepine, N., Labat, K., and Ricarte, P. (2010). $\mathrm{CO}_{2}$ plume imaging using 3d pre-stack stratigraphic inversion: A case study on the sleipner field. First Break, $(30): 91-96$.

Coueslan, M. L. (2007). Processing and interpretation of time-lapse vertical seismic profile data from the penn west $\mathrm{CO}_{2}$ monitoring project. MSc Thesis, University of Calgary.

Cox, M. J. (1999). Static Corrections for Seismic Reflection Surveys.

Dashtgard, S., Buschkuehle, M., Berhane, M., and Fairgrieve, B. (2006). Local-scale baseline geological report for the Pembina-Cardium $\mathrm{CO}_{2}$-enhanced oil recovery site. Alberta Geological Survey, (Alberta Energy and Utilities Board).

Davis, T. L., Terrell, M. J., Benson, R. D., Cardona, R., Kendall, R. R., and Winarsky, R. (2003). Multicomponent seismic characterization and monitoring of the $\mathrm{CO}_{2}$ at Weyburn Field,Saskatchewan. TLE, (22):696-697.

Eastwood, J., Lebel, J. P., Dilay, A., and Blakeslee, S. (1994). Seismic monitoring of steam-based recovery of bitumen. TLE, (4):242-251.

Eiken, O., Brevik, I., R., A., Lindeberg, E., and Fagervik, K. (2000). Seismic monitoring of $\mathrm{CO}_{2}$ injected into a marine acquifer. SEG Exp. Abst.

Greaves, R. J. and Fulp, T. J. (1987). Three-dimensional seismic monitoring of an enhanced oil recovery process. Geophysics, (52):1175-1187.

Hart, D. I. (1997). Enhancing wavelet stability with surface-consistent deconvolution. SEG Exp. Abst.

Helgerud, M. B., Miller, A. C., Johnston, D. H., Udoh, M. S., Harris, C., Jardine, B. G., 
and Aubuchon, N. (2011). 4d in the deepwater gulf of mexico: Hoover, madison, and marshall fields. TLE, (30):986-1079.

Henley, D. C. (2003). Coherent noise attenuation in the radial trace domain. Geophysics, (68):1408-1416.

Hileman, J. A., Embree, P., and Pflueger, J. (1968). Automated static corrections. Geophys. Prosp., (16):326-358.

Hitchon, B. (2009). Pembina Cardium $\mathrm{CO}_{2}$ monitoring pilot: $\mathrm{A} \mathrm{CO}_{2}$-EOR project, Alberta, Canada. Geoscience publishing.

Jack, I. (1998). Time-lapse seismic in reservoir management. SEG short course notes.

Johnstad, S. E., Uden, R. C., and Dunlop, K. N. B. (1993). Seismic reservoir monitoring over the oseberg field. FB, (11):177-185.

Johnston, D. H. (2013). Practical Applications of Time-lapse Seismic Data. SEG DISC, (16).

Karl, J. H. (1989). An Introduction to Digital Signal Processing.

Kragh, E. and Christie, P. (2002). Seismic repeatability, normalized rms, and predictability. TLE, (21):640-647.

Lancsoz, C. (1961). Linear differential operators.

Landrø, M. (1999). Repeatability issues of 3-d vsp data. Geophysics, (64):1673-1679.

Larner, K. L., Gibson, B., Chambers, R., and Wiggins, R. A. (1979). Simultaneous estimation of residual statics and crossdip time corrections. Geophysics, (44):11751192. 
Lawton, D., Coueslan, M., Chen, F., Bland, H., Jones, M., Gallant, E., and Bertram, M. (2005). Overview of the Violet Grove $\mathrm{CO}_{2}$ seismic monitoring project. CREWES report, (17):1-24.

Levin, S. A. (1989). Surface-consistent deconvolution. Geophysics, (54):1123-1133.

Li, G. (2003). 4D seismic monitoring of $\mathrm{CO}_{2}$ flood in a thin fractured carbonate reservoir. TLE, (22):690-695.

Lumley, D., Adams, D. C., Meadows, M., Cole, S., and Wright, R. (2003). 4d seismic data processing issues and examples. SEG Exp. Abst., (22):1394-1397.

Lumley, D. E. (1995). 4-d seismic monitoring of an active steamflood. SEG Exp. Abst., $(14): 203-206$.

Lumley, D. E. (2001). Time-lapse seismic reservoir monitoring. Geiophysics, (1):50-53.

Lumley, D. E., Behrens, R. A., and Wang, Z. (1997). Assessing the technical risk of a 4D seismic project. TLE, (16):1287-1294.

Montana, C. A. and Margrave, G. F. (2006). Surface-consistent gabor deconvolution. SEG Exp. Abst.

Morgan, N. A. (1970). Wavelet maps: A new tool for reflection seismograms. Geophysics, (35):447-460.

Morley, L. and Claerbout, J. (1983). Predictive deconvolution in shot-receiver space. Geophysics, (48):515-531.

Nedlin, G. (1985). Surface-consistent suppression of multiples. Geophysics, (50):17791783.

Nur, A. (1982). Seismic imaging in enhanced recovery. SPE. 
Patterson, N. R. (1957). Geology of the Pembina field, Alberta. Bulletin of the AAPG, (41):937-949.

Poggiagliolmi, E., Berkhout, A. J., and Boone, M. M. (1982). Phase unwrapping, possibilities and limitations. Geophysical Prospecting, (30):281-291.

Porter-Hirsche, J. L. and Hirsche, K. W. (1998). Repeatability study of land data acquisition and processing for time lapse seismic. SEG Exp. Abst.

Pullin, N., Matthews, L., and Hirsche, K. (1987). Techniques applied to obtain very high resolution 3-D seismic imaging at an Athabasca tar sands thermal pilot. TLE, (12):10-15.

Rennie, J., Alexandre, R., and Ronen, S. (1997). Sensitivity of repeated 3-d seismic surveys to geometry variations - a controled experiment. SEG Exp. Abst.

Rickett, J. E. and Lumley, D. E. (2001). Cross-equalization data processing for timelapse seismic reservoir monitoring: A case study from the gulf of mexico. Geophysics, (66):1015-1025.

Robinson, E. A. and Treitel, S. (1980). Geophysical signal analysis. Prentice-Hall, Inc.

Ross, C. P. and Altan, M. S. (1997). Time-lapse seismic monitoring: Some shortcomings in nonuniform processing. TLE, (16):931-937.

Ross, C. P., Cunningham, G. B., and Weber, D. P. (1996). Inside the crossequalization black box. TLE, (15):1233-1240.

Schissele, E., Forgues, E., Echappe, J., Meunier, J., de Pellegars, O., and Hubans, C. (2009). Seismic repeatability: Is there a limit? EAGE Exp. Abst.

Schneider, W. A. (1971). Developments in seismic data processing and analysis. Geophysics, (36):1043-1073. 
Shah, P. (1973). Use of wavefront curvature to relate seismic data with subsurface parameters. Geophysics, (38):812-825.

Sheriff, R. E. (2002). Encyclopedic Dictionary of Exploration Geophysics.

Taner, M. T. and Coburn, K. W. (1980). Surface consistent estimation of source and receiver response functions. 50th Annual International SEG Meeting.

Taner, M. T. and Koehler, F. (1981). Surface consistent corrections. Geophysics, (46):1722.

Taner, M. T., Koehler, F., and Alhilali, K. A. (1974). Estimation and correction of near-surface time anomalies. Geophysics, (39):441-463.

Taner, M. T., Lee, L., and Baysal, E. (1991). Static corrections: time, amplitude, and phase. SEG Exp. Abst.

Wang, Z. and Nur, A. (1989). Effect of temperature on wave velocities in sandstones and sands with heavy hydrocarbons, in nur, a. and wang, z., eds., seismic and acoustic velocities in reservoir rocks. Soc. Expl. Geophysics.

White, D. (2009). Monitoring $\mathrm{CO}_{2}$ storage during EOR at the Weyburn-Midale Field. TLE, (28):838-842.

Wiggins, R. A., Larner, K. L., and Wisecup, R. D. (1976). Residual static analysis as a general linear inverse problem. Geophysics, (41):922-938.

Williams, G. and Chadwick, A. (2012). Quantitative seismic analysis of a thin layer of $\mathrm{CO}_{2}$ in the sleipner injection plume. Geophysics, (77):245-256.

Yilmaz, O. (2001). Seismic data analysis. 
Yu, G. (1985). Offset-amplitude variation and controlled-amplitude processing. Geophysics, (50):2697-2708.

Zweigel, P., Arts, R., Bidstrup, T., Chadwick, A., and Gregersen, U. (2001). Results and experiences from the first industrial-scale underground $\mathrm{CO}_{2}$ sequestration case (Sleipner Field, North Sea). AAPG Exp. Abst. 